\title{
A Mixed Methods study of Early Intervention Practitioners' Attitudes towards the Implementation of Evidence Based Practices in England and Wales
}

\author{
White, Joanna
}

How to cite:

White, Joanna (2019) A Mixed Methods study of Early Intervention Practitioners' Attitudes towards the Implementation of Evidence Based Practices in England and Wales. Doctoral thesis, Swansea University. http://cronfa.swan.ac.uk/Record/cronfa51971

Use policy:

This item is brought to you by Swansea University. Any person downloading material is agreeing to abide by the terms of the repository licence: copies of full text items may be used or reproduced in any format or medium, without prior permission for personal research or study, educational or non-commercial purposes only. The copyright for any work remains with the original author unless otherwise specified. The full-text must not be sold in any format or medium without the formal permission of the copyright holder. Permission for multiple reproductions should be obtained from the original author.

Authors are personally responsible for adhering to copyright and publisher restrictions when uploading content to the repository.

Please link to the metadata record in the Swansea University repository, Cronfa (link given in the citation reference above.) 


\section{A Mixed Methods study of Early Intervention Practitioners' Attitudes towards the Implementation of Evidence Based Practices in England and Wales.}

Thesis submitted to Swansea University in fulfilment of the requirements for the Degree of Doctor of Philosophy

By Joanna White

College of Human and Health Sciences 2019 


\section{SUMMARY (ABSTRACT)}

A key element of future plans for mental health services in England and Wales will be evidence based service models and approaches. This study examined attitudes towards the implementation of evidence based practices in a sample of early intervention practitioners. Evidence-based practice integrates individual practitioner expertise with the best available evidence while also considering the values and expectations of clients.

This study used a mixed methods sequential explanatory design to assess evidence based practices in mental health within early intervention practitioners. The quantitative component consisted of a survey using a demographic questionnaire and the Evidence Based Attitudinal Scale (Aarons, 2004) from $n=70$ practitioners, a response rate of $64 \%$. The qualitative component of the study was semi-structured research interviews. Eighteen participants were selected via a purposive sampling using a range of criteria. Participants' were asked questions about their everyday experiences of implementation issues in their early intervention teams focusing on aspects such as sustainability and fidelity.

The results of the study show that attitudes towards adoption of evidence based practices can be assessed within early intervention teams. The main finding from the quantitative phase of the study is that the Evidence Based Practice Attitudinal Scale requirement and openness scales are strong predictors of attitudes. The semi-structured interview data provided a rich picture of the sustainability of evidence based practice in mental health and the challenges and opportunities that this brings such as barriers and protection of early intervention services to adopting evidence based practices.

Future research should examine all stakeholders' views on implementing evidence based practices as this research has shown that a whole systems approach is required. The move towards service user involvement in research and the possibilities of examining implementation in more democratic ways must also be explored further. 


\section{DECLARATION AND STATEMENTS}

\section{DECLARATION}

This work has not previously been accepted in substance for any degree and is not being concurrently submitted in candidature for any degree.

Signed (candidate)

Date

\section{STATEMENT 1}

This thesis is the result of my own investigations, except where otherwise stated. Other sources are acknowledged with explicit references, a full reference list is appended.

Signed (candidate)

Date

\section{STATEMENT 2}

I hereby give consent for my thesis, if accepted, to be available for photocopying and for inter-library loan, and for the title and summary to be made available to outside organisations.

Signed (candidate)

Date 


\section{ACKNOWLEDGEMENTS}

I would like to take this opportunity to express my thanks and appreciation to a number of people that have assisted and supported me with this work.

My supervisor Professor Michael Coffey; his guidance and constructive suggestions along with his personal hands-on experience of the subject matter has been invaluable. He has inspired me with confidence throughout, especially with some of the difficult chapters of my thesis.

The late Professor Damon Berridge has been immensely supportive with the statistical aspect of my thesis, the data interpretation and analysis would have been more challenging without his continuous attention to detail.

Professor Peter Huxley who appointed me to my first research position some years ago, he has instilled in me good research practice principles that have stood me well throughout this work, his expertise with aspects of my work are truly appreciated.

My thanks and appreciation is also extended to, Professor Gary Rolfe who also gave some assistance with this work.

I would also like to thank Stephen McGowan, Alyson Leeks from the (IRIS network) and Dr Ewan Hails (1000 Lives Wales) for linking my research with early intervention practitioners in England and Wales.

My appreciation and thanks must be extended to all of the early intervention practitioners for completing my survey, and the participants that gave their time to be interviewed. I am so grateful to them all.

Last but not least thank you to my husband Jonathan and my parents for their years of enduring support. 


\section{TABLE OF CONTENTS}

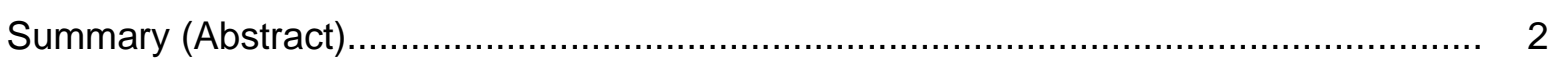

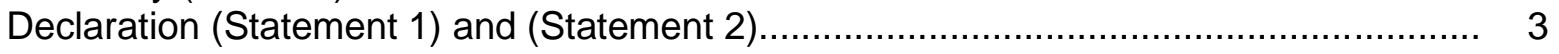

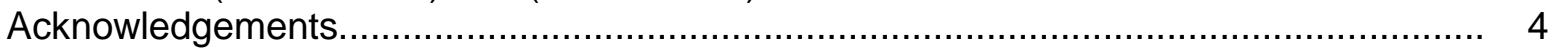

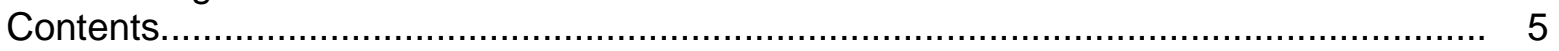

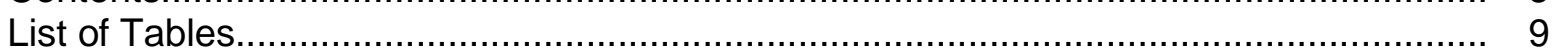

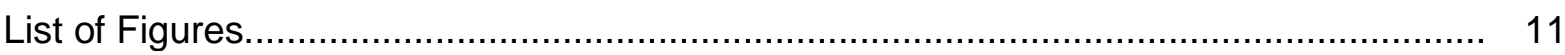

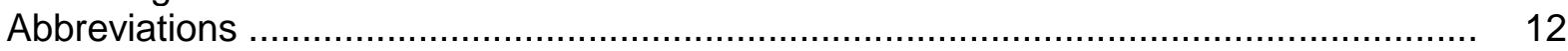

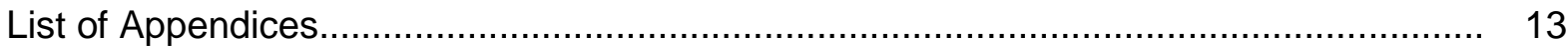

CHAPTER 1

INTRODUCTION

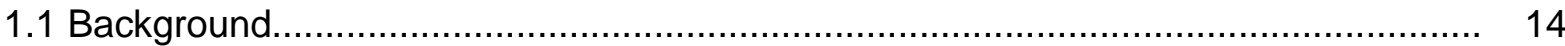

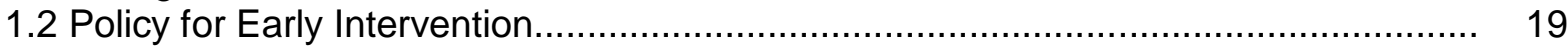

1.3 Key Concepts in Organisational Social Context..................................................... 24

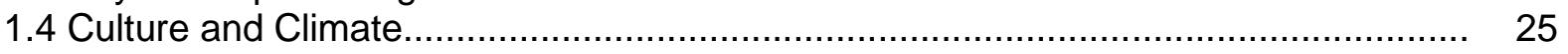

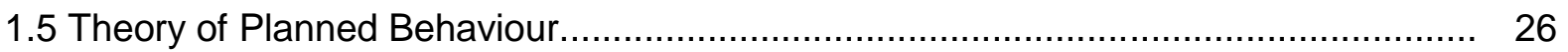

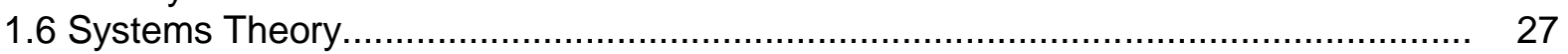

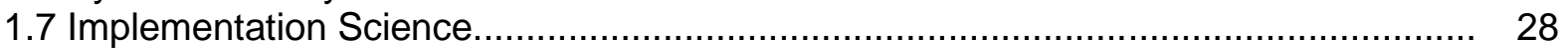

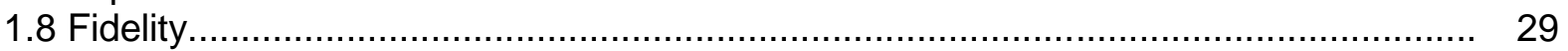

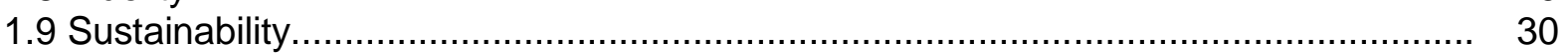

1.10 Implementing Evidence Based Practice in Mental Health..................................... 30

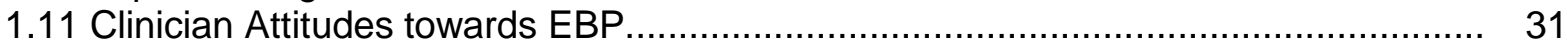

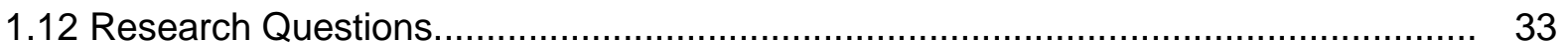

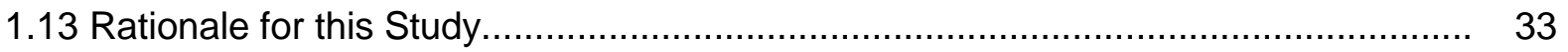

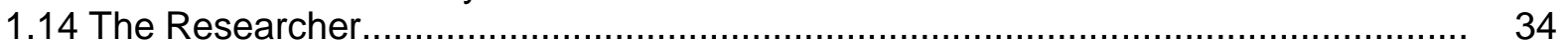

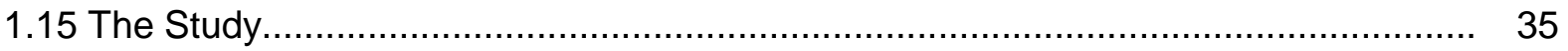

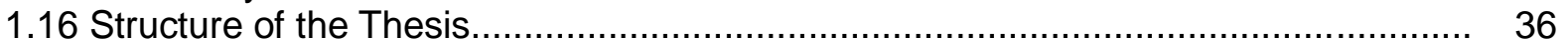

\section{CHAPTER 2}

\section{LITERATURE REVIEW}

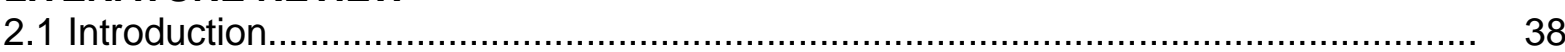

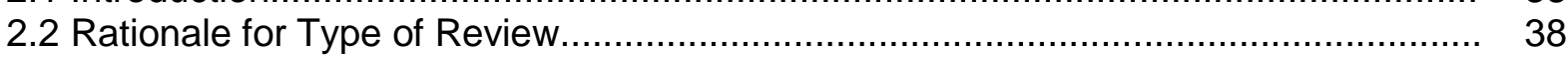

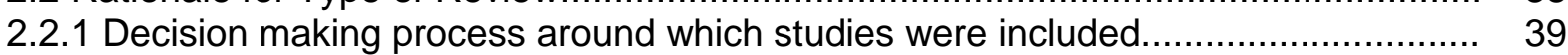

2.2.2 Types of Studies (Eligibility Criteria) ................................................................ 39

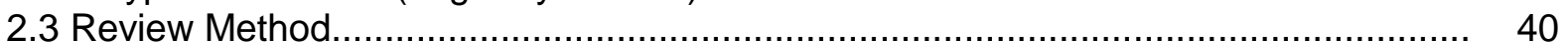

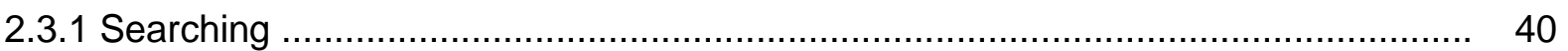

2.4 The Concept of Early Intervention in Psychosis (EIP) ......................................... 43

2.5 The Effectiveness of Early Intervention Teams..................................................... 43

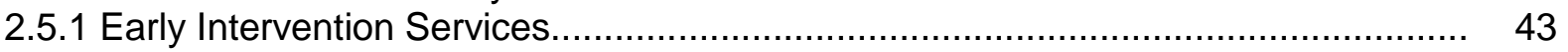

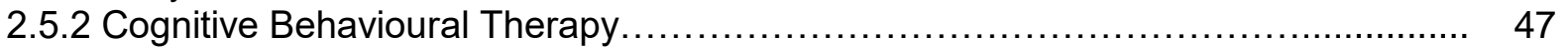

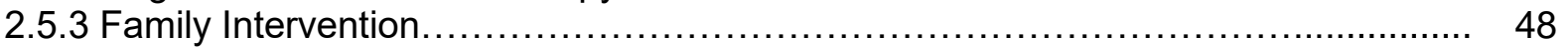

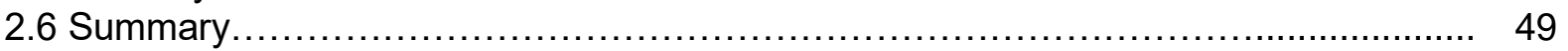

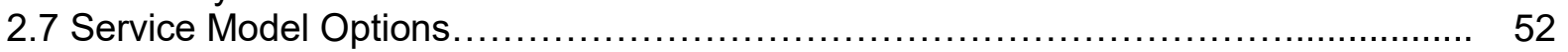

2.7.1 Traditional Community Mental Health Team (CMHT) Model............................... 52

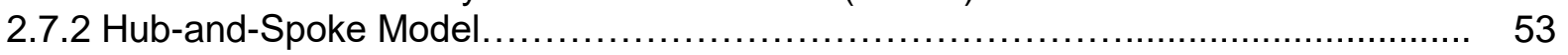

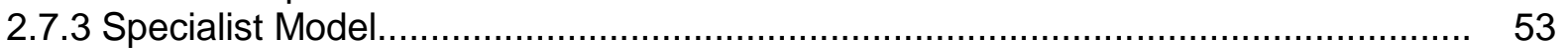

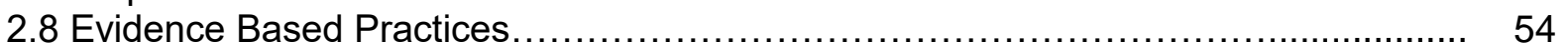

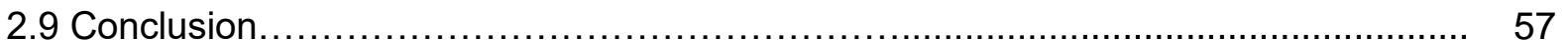




\section{CHAPTER 3 \\ METHODS}

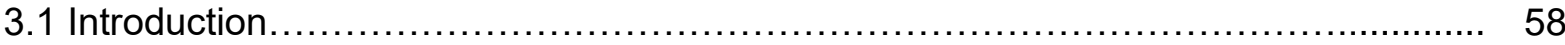

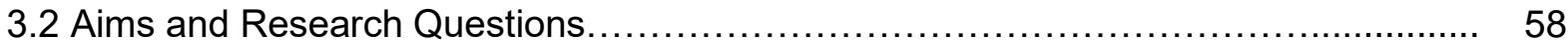

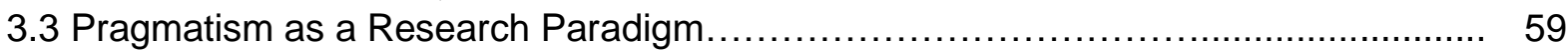

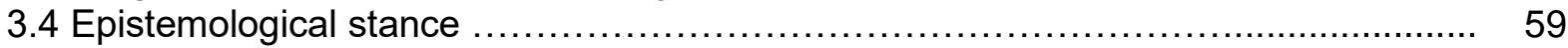

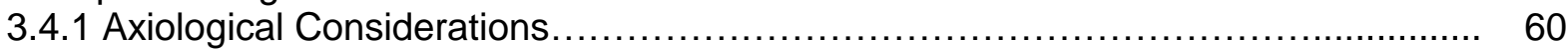

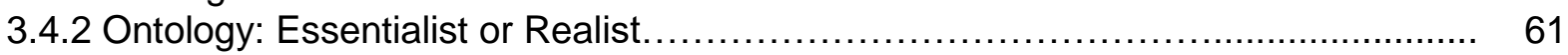

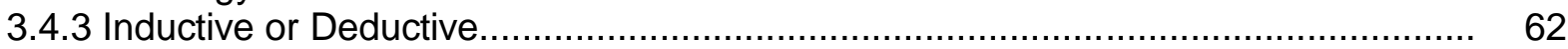

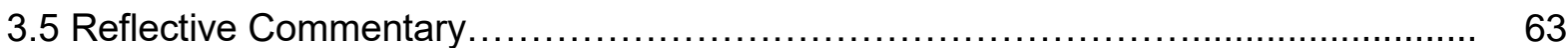

3.6 Three Communities Continua, not Dichotomies .......................................... 65

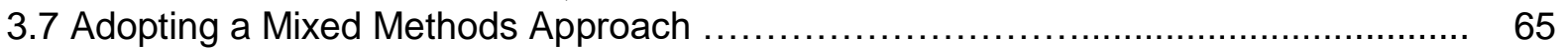

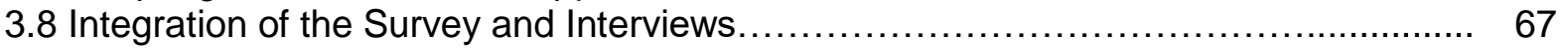

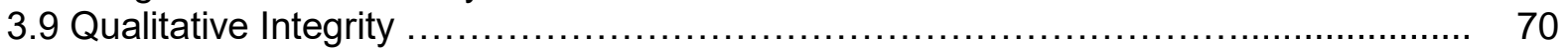

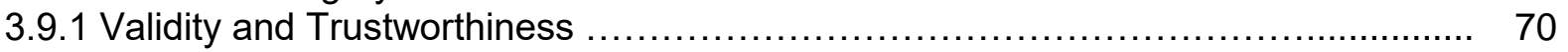

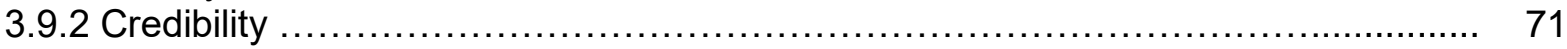

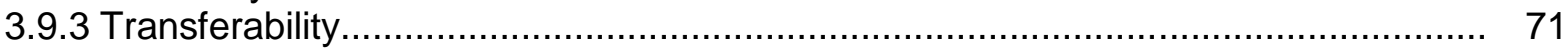

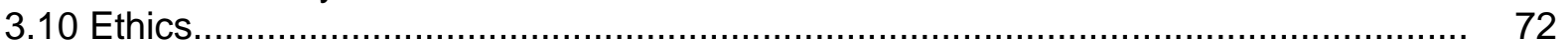

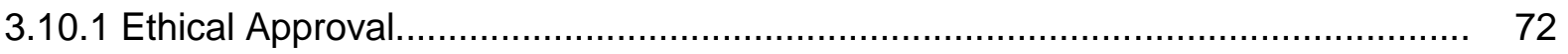

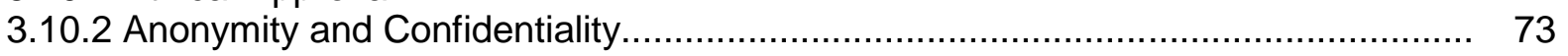

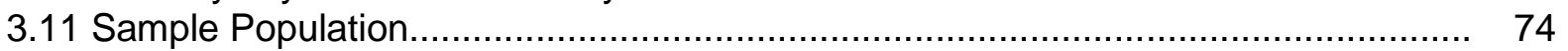

3.12 Negotiating Access to Early Intervention Practitioners............................... 75

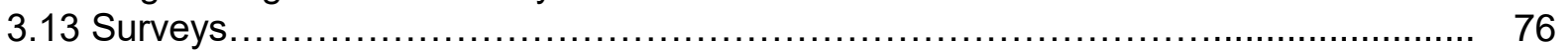

3.13.1 Design, Conduct, Advantages and Disadvantages of Surveys ......................... 77

3.14 Evidence Base Practice Attitudinal Scale (EBPAS, Aarons, 2004) ...................... 79

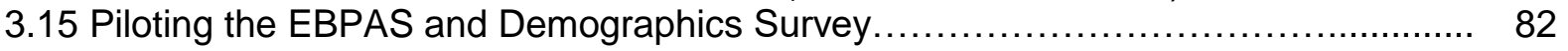

3.16 Research Interviews in Qualitative Research................................................. 83

3.17 Telephone Interviewing in Qualitative Research.............................................. 86

3.18 Data Collection and Transcription................................................................. 87

3.19 The Online Survey Questions: (Evidence Based Practice Attitudinal Scale and

Demographics).

3.20 Data Analysis.

88

88

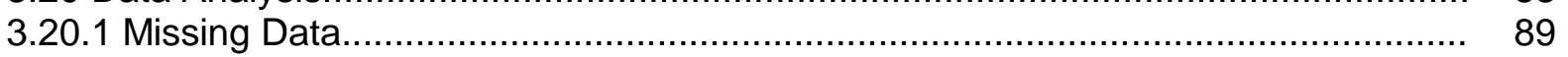

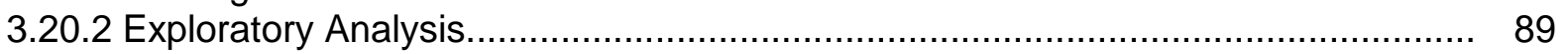

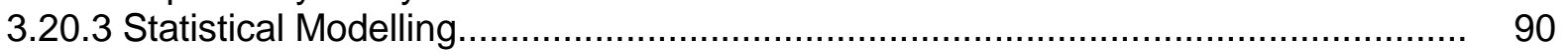

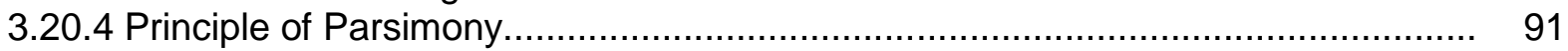

3.20.5 Modelling: Building Processing and Selection............................................... 92

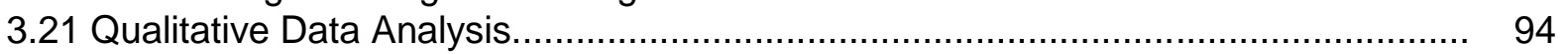

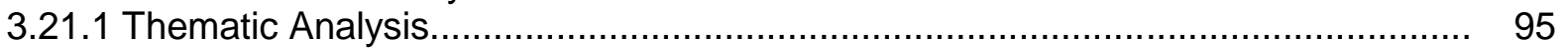

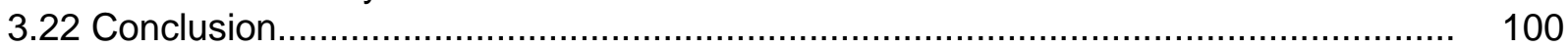

\section{CHAPTER 4}

\section{QUANTITATIVE FINDINGS}

4.1 Introduction...

4.2 Data Description / Exploratory Analysis

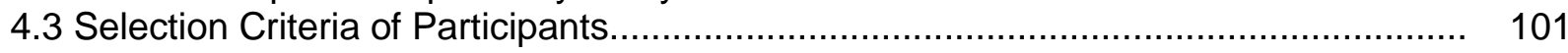

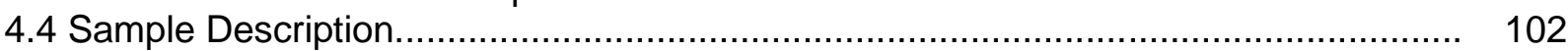

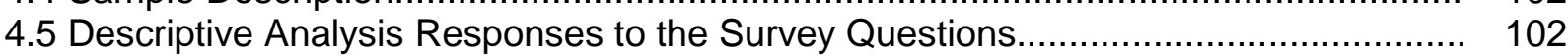

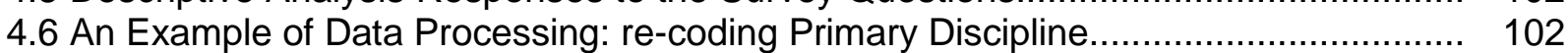

4.7 Summary of Demographic Variable Used in the Survey...................................... 103

4.8 Descriptives on the Evidence Based Attitudinal Scale (EBPAS) ............................. 104

4.9 Bivariate Analysis: Examining the Relationship between Two Categorical variables.

4.9.1 Age with how many years' Experience you have working in Mental Health 
Services.

4.9.2 Highest level of Education with Primary Discipline

4.9.3 Highest level of Education with Types of Services Provided by Service Model: Inpatient.

4.9.4 Types of Services Provided by your Service Model: Inpatient with

Outpatient.

4.9.5 Types of Services Provided by your Service Model: Inpatient with Day

Treatment.

4.9.6 Does your Organisation Adhere to the following Policies: Welsh Psychological

Therapies with Practice Policy Mental Health Wales's Measure

4.9.7 How long has your Early Intervention Team been Established with does your

Organisation Adhere to the Following Policies: Wales's Mental Health

Measure.

4.10 Level of Familiarity with Evidence Based Practice and Empirical Supported

Treatments.

4.11 Statistical Modelling....

4.12 Regression Analysis

4.12.1 Factor 1 of Analysis 3, EBPAS Scale Requirement.

4.12.2 Factor 2 of Analysis 3, EBPAS Openness with Primary Discipline

'Psychiatry'...

120

4.13 EBPAS Attitudinal score with Practice Policy Welsh Psychological Therapies (WPT).

4.13.1 EBPAS Attitudinal Score with Practice Policy Welsh Psychology Therapies

(WPT)

4.14 Statistical Modelling and Interactions

4.14.1 Demographics

4.15 Organisational Variables.

4.15.1 Models of Gender and Policy Main Effects plus Gender and Policy

Interaction.

4.16 Finding the most Parsimonious Model of the Requirements Scale Defining the Full Model.

4.16.1 Goodness of Fit.

4.16.2 The most Parsimonious Model: final Model for EBPAS Total Score.

4.17 Summary of Regression Analysis and Interactions.

4.18 Summary of Findings.

\section{CHAPTER 5}

\section{QUALITATIVE FINDINGS}

5.1 Introduction.

5.2 Selection of Interview Participants

5.3 Analysis of Data.

5.4 Sustaining Evidence Based Practices

5.5 Category 1: What constitutes Evidence Based Practice in relation to early

intervention.

5.6 Category 1: Specialised Practice

5.7 Category 2: Champions

5.7.1 Subcategory 1 : Senior

5.8 Summary of Theme One.

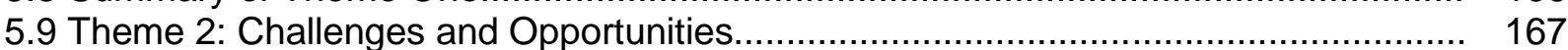

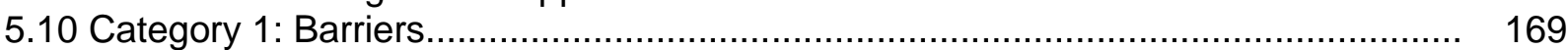

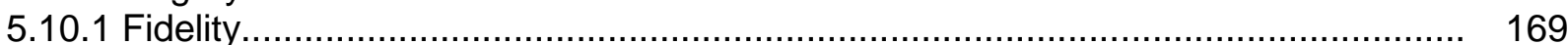

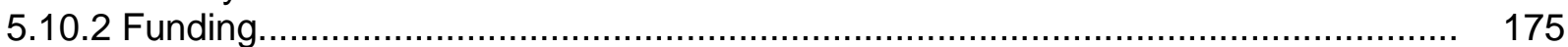

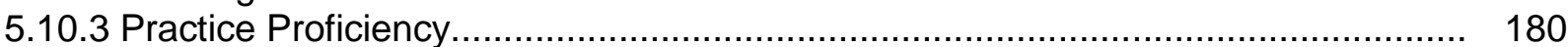

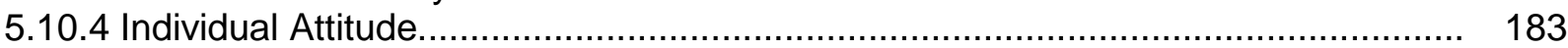


5.11 Category 2: Protection of Early Intervention in Psychosis Services.

5.11.1 Outcomes

5.12 Summary for Theme Two.

5.13 Summary of Key Findings from Data Analysis.................................................... 190

5.14 Conclusion.

CHAPTER 6

DISCUSSION AND INTEGRATION OF FINDINGS

6.1 Introduction

6.2 Quantitative Findings.

6.2.1 EBPAS Requirement Scale: Older vs. Younger Respondents.....................................

6.2.2 EBPAS Openness Scale: Psychiatrists and Nurses....................................... 197

6.2.3 EBPAS Score with Primary Discipline................................................................. 199

6.2.4 Primary Discipline with Service Models............................................................. 201

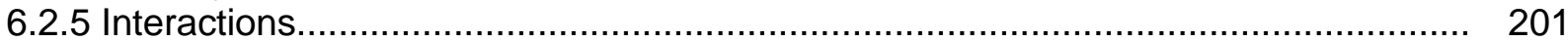

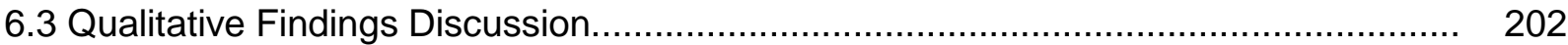

6.4 Sustaining Evidence Based Practices: Evidence and Context................................. 202

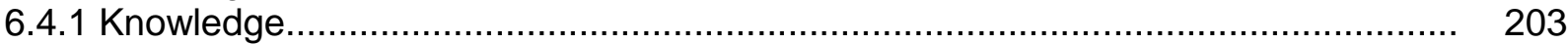

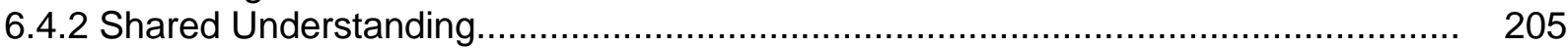

6.4.3 Transformational Leaders: Champion Leads on Senior Management.................... 206

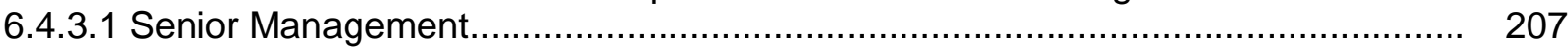

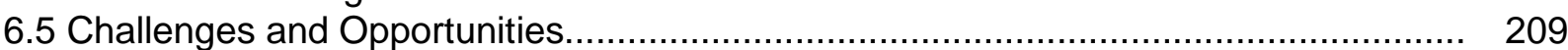

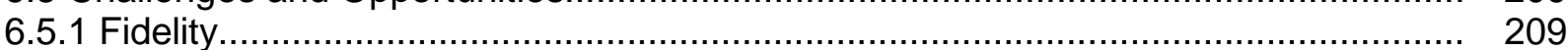

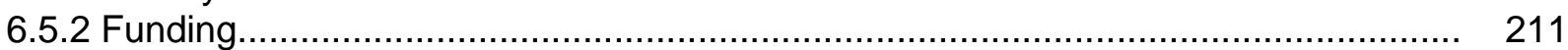

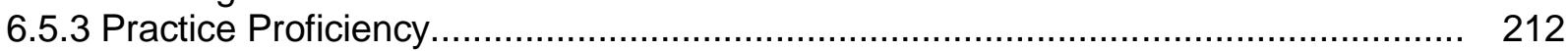

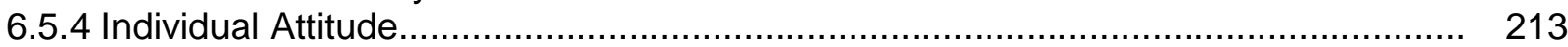

6.5.5 Protection of Early Intervention Services...................................................... 213

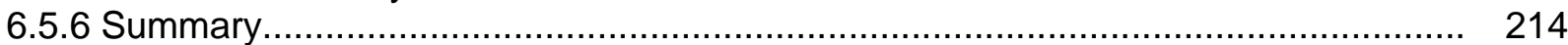

6.6 Integration of Quantitative and Qualitative Findings............................................ 215

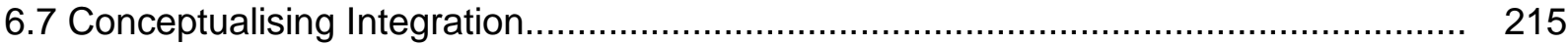

6.8 Mixed Methods Matrix (Integration of Quantitative and Qualitative Findings)............. 216

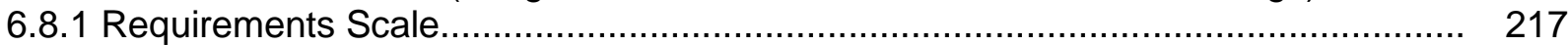

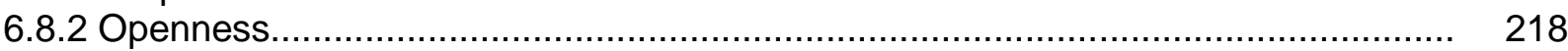

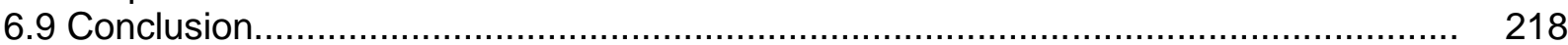

CHAPTER 7

CONCLUSION

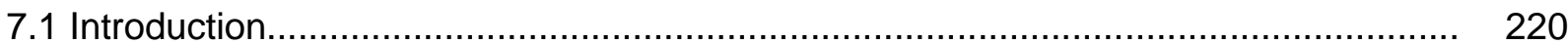

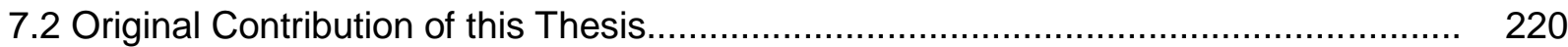

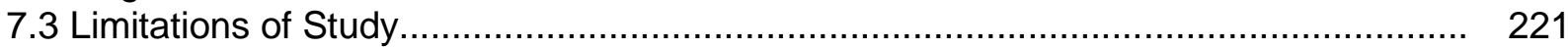

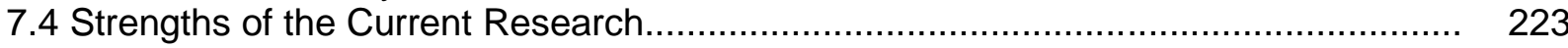

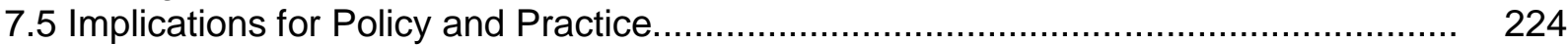

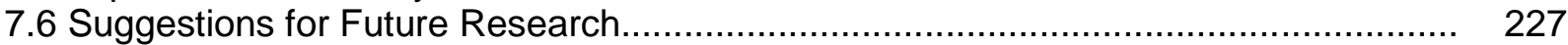

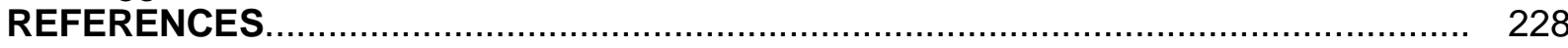

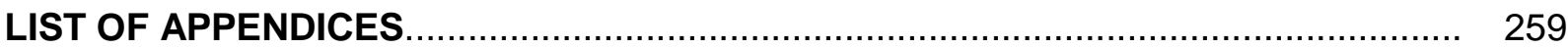




\section{LIST OF TABLES}

Table 1

Table 2

Table 3

Table 4

Table 5

Table 6

Table 7

Table 8

Table 9

Table 10

Table 11

Table 12

Table 13

Table 14

Table 15

Table 16

Table 17

Table 18

Table 19.1

Table 19.2

Table 20

Table 21

Table 22

Table 23

Table 24

Table 25

Table 26

Table 27

Table 28

Table 29
Example of Coding from Research

Age Descriptives

Recode Example, Original Variable - Primary Discipline............. 103

Final Recode of the Primary Discipline Variable....

105

Cross Tabulation Showing the Type of Service Provided by

Service Model Inpatient and Outpatient.

Cross Tabulation Showing Types of Services Provided by your

Service Model Inpatient with Day Treatment.

Comparison of Alphas

Overall Means, Standard Deviations, Item Total Correlations,

Eigenvalues, Internal Consistency Reliabilities, and Item

Loadings for Each of The Scales for

EBPAS

Age.

Factor 1 of Analysis 3, EBPAS Scale Requirement with

Organisation Adheres to Policy Implementation Guidance

(PIG).

Factor 1 of Analysis 3, EBPAS Scale Requirement with

Organisation Adheres to National Institute for Clinical

Excellence (NICE)

Factor 1 of Analysis 3, EBPAS Scale Requirement with

Organisation Adheres to Welsh Psychological Therapies

(WPT).

Factor 2 of Analysis 3, EBPAS Openness Scale with Primary

Discipline.

EBPAS Attitudinal Score with Primary Discipline

EBPAS Attitudinal Score with Practice Policy Welsh

Psychological Therapies (WPT)

Model 1 Gender Main Effects

125

Model 2 Age Main Effects.

126

Model 3 Gender and Age Main Effects..................................... 127

ANOVA Table between Age and Gender................................. 128

Model 4 Gender and Age Plus Interactions............................... 128

Frequencies on adherence to practice policies......................... 130

Model 2: Policy Main Effects

Model 3: Age and Policy Main Effects.....

132

Model 4: Age and Policy Main Effects Plus Interactions.

133

ANOVA: Age and Policy Main Effects Plus Policy Interaction

(PIG And Welsh and Mental Health

Measure).

ANOVA: Age and PIG Main Effects Plus Age by PIG

Interactions

ANOVA Age by Policy (1 Category PIG)

Model 6 Primary Discipline by Service Model (Inpatient) Main

Effects Plus Interactions.

Model 6 Primary Discipline by Service Model (Outpatient) Main

Effects Plus Interactions.

The Most Parsimonious Model of The Requirements

Scale 
Table 30 Comparison of Observed And Predicted / Expected for EBPAS

Requirement Scale.......................................................... 139

Table $31 \quad$ The most Parsimonious Model of the EBPAS Scale.................... 141

Table $32 \quad$ Selection of Interview Participants............................................. 148 


\section{List of Figures}

Figure 1

Figure 2

Figure 3

Figure 4

Figure 5

Figure 6

Figure 7

Figure 8

Figure 9

Figure 10

Figure 11

Figure 12

Figure 13

Figure 14

Figure 15

Figure 16

Figure 17

Figure 18

Figure 19
Traditional Model of Evidence Based Practice

56

An Alternative Model of Evidence Based Practice.

Deductive and Inductive Approaches to the Relationship Between Theory

And Research.

Explanatory Design: Participant Selection Model.....

EBPAS Divergence Scale: I Know Better than Academic

Researchers.

EBPAS Divergence Scale: Clinical Experience is more Important....

EBPAS Divergence Scale: Research Based Treatments / Interventions are

not Clinically Useful.....

EBPAS Divergence Scale: I would not use Manualised Therapy /

Interventions.

Clustered Bar Chart Age with how many years' Experience Working in

Mental Health Services.

Clustered Bar Chart Highest Level of Education With Primary

Discipline.

Clustered Bar Chart Highest Level of Education With Types of Services

Provided by Service Model: Inpatient.

A Clustered Bar Chart Showing does your Organisation Adhere To The

Following Policies: Welsh Psychological Therapies with Practice Policy Mental Health Wales's Measure.

A Clustered Bar Chart Showing how long has your Early Intervention Team

Been Established with does your Organisation Adhere to the following

Policies: Wales's Mental Health Measure.

A Pie Chart showing Familiarity of EBP and EST.

A Line Chart showing Age by Gender Interaction.

Age by Policy Interaction...

Goodness of Fit for EBPAS Requirements.

135

Theme 1: Visual Representation of Theme and Category.

139

149

Theme 2: Visual Representation of Theme and Category 


\section{ABBREVIATIONS}

$\begin{array}{ll}\text { Abbreviation } & \text { Description } \\ \text { AO } & \text { Assertive Outreach } \\ \text { AOF } & \text { Annual Operating Framework } \\ \text { BPS } & \text { British Psychological Society } \\ \text { CAMHS } & \text { Child and Adolescent Mental Health Services } \\ \text { CCGs } & \text { Clinical Commissioning Groups } \\ \text { CFA } & \text { Confirmatory Factor Analysis } \\ \text { CMHT } & \text { Community Mental Health Team } \\ \text { COAST } & \text { Croydon Outreach and Assertive Support Team } \\ \text { DoH } & \text { Department of Health } \\ \text { DUP } & \text { Duration of Untreated Psychosis } \\ \text { EBPAS } & \text { Evidence Based Attitudinal Scale } \\ \text { EBPs } & \text { Evidence Based Practices } \\ \text { EFA } & \text { Exploratory Factor Analysis } \\ \text { EI } & \text { Early Intervention } \\ \text { EIP } & \text { Early Intervention in Psychosis } \\ \text { EPPIC } & \text { Early Psychosis Prevention and Intervention Centre } \\ \text { ESTs } & \text { Empirical Supported Treatments } \\ \text { IRIS } & \text { Initiative to Reduce the Impact of Schizophrenia } \\ \text { MHPIG } & \text { The Mental Health Policy Implementation Guideline } \\ \text { MHWM } & \text { Mental Health Wales Measure } \\ \text { NICE } & \text { The National Institute for Health and Care Excellence } \\ \text { NMC } & \text { Nursing and Midwifery Council } \\ \text { NRES } & \text { National Research Ethics Service } \\ \text { NSF } & \text { National Service Framework } \\ \text { OTP } & \text { Optimal Treatment Project } \\ \text { REC } & \text { Research Ethics Committee } \\ \text { SMI } & \text { Severe Mental Illness } \\ \text { TAU } & \text { Treatment as Usual } \\ \text { TPB } & \text { Theory of Planned Behaviour } \\ \text { WG } & \text { Welsh Government } \\ \text { WPT } & \text { Welsh Psychological Therapies } \\ \text { WTE } & \text { Working Time Equivalent } \\ & \\ & \end{array}$




\section{LIST OF APPENDICES}

Appendix 1

Search Strategy................................................... 259

Appendix $2 \quad$ Demographics and EBPAS............................................ 260

Appendix $3 \quad$ Assumptions of Normality............................................. 269

Appendix $4 \quad$ Semi-Structured Interview Schedule................................. 271

Appendix $5 \quad$ Research Ethics Committee Letter (REC)......................... 279

Appendix 6 National Research Ethics Service

(NRES) ............................................................... 280

Appendix $7 \quad$ Participant Consent........................................................ 281

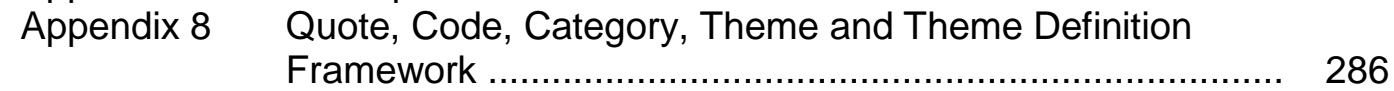

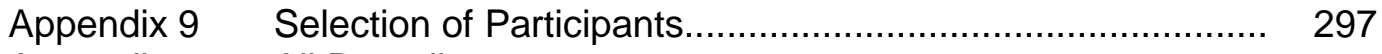

Appendix 10 All Recoding.............................................................. 298

Appendix 11 Summary of Demographic Variables Used in the

Survey....................................................................... 303

Appendix 12 Summary of Evidence Based Practice Attitudinal Scale Measure........................................................ 305

Appendix 13 Regressions.............................................................. 308

Appendix $14 \quad$ Models 1 to 4 Primary Discipline...................................... 314

Appendix 15 Mixed Methods Matrix.................................................... 319 


\section{CHAPTER 1: Introduction}

\subsection{Background}

This thesis examines the attitudes and experiences of practitioners towards implementation of evidence based practice in English and Welsh early intervention mental health services. I set out to generate evidence for the purpose of developing an in-depth understanding of implementation of evidence based practices by deploying an explanatory sequential mixed methods design (Creswell \& Plano Clark, 2011) for the purpose of building a comprehensive picture of how evidence is understood and used to develop and improve a key area of mental health service delivery. I was seeking to first measure attitudes towards evidence implementation of lead practitioners and followed this by conducting in-depth research interviews with a range of lead practitioners to examine everyday experiences of implementation issues.

The NHS planned on spending £11.9 billion on mental health in 2017/ 2018 (Five Year Forward Review, 2016). The cost of mental ill health to the economy, the NHS and society as a whole is ( $£ 105 \mathrm{bn}$ ) a year (Department of Health, 2016) suggesting a real need for improvements in the delivery of effective evidence-based care. A key element of the future plans for mental health services in England will be, 'evidence based service models and approaches' (Department of Health, 2009 p 100). Community mental health services in England and Wales are delivered by multidisciplinary teams, led by a team manager. Team managers are key individuals in ensuring that their services are organised according to the best evidence and deliver care and treatments that are also based on the best evidence (Lloyd \& King, 2009). These managers are frequently professionals who have taken on a managerial role, often without any specific training for it. Managers come from a variety of disciplinary backgrounds. They may be nurses, occupational therapists, psychologists, psychiatrists or social workers. According to Gilbert (2003) mental health services' greatest assets are the practitioners who provide care and their managers. From their own clinical experience, new managers are aware of the 
practicalities and demands of everyday work, but now they have another set of demands from higher up the system to which they must also respond. Managers are regularly expected to implement, and be accountable for, a vast number of changes (Arnold, 2005). This can occur without benefit of a comprehensive understanding of the processes involved in initiating, implementing, and sustaining change.

Looking at evidence based practice in mental health settings Drake et al., (2008) note that practices that have been demonstrated to be effective by clinical services research could improve the lives of many people if they were widely adopted in routine health care settings. Evidence-based practice integrates individual practitioner expertise with the best available evidence while also considering the values and expectations of clients. In this thesis the attitudes and implementation of early intervention practitioner leaders to evidence based practice is examined.

Most of the research concerning evidence based practice, evidence based management and evidence informed practice has been conducted discretely within the disciplines of management (business and economics), medicine and allied professionals, and social care (Crilly, 2009). Crilly, Jashapara and Ferlie (2010) have reviewed the management literature; Nutley and colleagues have reviewed the health care literature; and Pawson, McDonald and Huxley et al., $(2003,2009)$ have reviewed the evidence base and research culture in social care and they found that different types of knowledge overlap but are by no means the same as the types of knowledge used in social work practice. Social workers tend to favour the constructivist paradigm (favouring reflexivity and narrative) as opposed to the positivist or post-positivist position.

The mental health field brings together the different disciplines of psychology, psychiatry, social work and nursing in the same context. Each of these disciplines has different traditions of empirical research in community services, and each draws differentially on qualitative and quantitative methods, and 
underlying theories of knowledge. Without a doubt, it could be argued that it would be hard to find anywhere that encompassed such a wide range of opinion about the nature of evidence, the means of implementing it, and the forms of justification for beliefs about 'what works' in practice than a multidisciplinary community mental health team (Davies, Nutley \& Smith, 2004).

User-led research has brought the service user view of these matters to the fore Sweeney, Beresford and Faulkner et al., (2009). User involvement and userresearch activity are possibly more developed in mental health providing an ideal opportunity to add experimental knowledge into the evidence mix.

Looking at evidence based practice in mental health settings Drake, Torrey and McHugo (2008) notes that practices that have been demonstrated to be effective by clinical services research could improve the lives of many people if they were widely adopted in routine health care settings. Most evidence-based interventions never become implemented in real-world practice despite a substantial focus on implementation of evidence-based psychological interventions (Haines, Kuruvilla, Borchert, 2004; McHugh \& Barlow, 2010).

Early intervention teams promote early detection and engagement to reduce the duration of untreated psychosis (DUP). They employ specialist staff to provide a range of interventions and anti-psychotic medications, tailored to the needs of young people with a view to facilitating recovery. Early intervention teams are distinguished by their cultural sensitivity to the unique needs of younger adults, their focus on families, and their attention to the impact of interrupted development and the social consequences of serious mental illness (NHS Confederation, 2011). We have seen the establishment of a specialised service model that provides evidence-based interventions for treating psychosis in the early phase and at a relatively young age (14-35 years old). An increasing body of evidence supports this approach as more effective than the traditional generic community mental health team approach (Bird \& Premkumar et al., 2011). This includes evidence that early intervention for psychosis results in a 
better course for reducing illness, fewer symptoms at eight years on and halving of the suicide rate (Mihalopoulos \& Harris et al., 2009).

Early Intervention in Psychosis (EIP) has been a radical service reform stimulated by a growing dissatisfaction from young people and their families to the 'one size fits all' approach of the late 1990s when ineffectual community services too often failed to give people good and timely support and resulted in crisis responses, hospitalisations, poor outcomes, and long term dependency on social services (IRIS Guidelines, 2012). As a result EIP became a government priority via the Department of Health (1999). The Initiative to reduce the impact of schizophrenia (IRIS) was established in the West Midlands, as a multidisciplinary group of expertise drawn together to improve local service provision. Subsequently policy commitment and an increasingly robust evidence base encouraged the establishment of EIP service model in most parts of England. From two teams supporting around 80 people in 1998, the capacity grew to about 150 teams providing care at any one time for about 22,000 people by March 2010 (Early Intervention in Psychosis IRIS Network, 2012). The implementation of new community mental health teams such as early intervention and crisis resolution teams and assertive outreach teams in England and Wales is geographically variable. The Policy Implementation Guidance (DoH, 2001 p.55) states that, "ideally each Early Intervention Service should manage 150 new cases per year and have a total caseload of approximately 450. It is envisaged that each early intervention service will cater for a population of around one million people. An understanding of local epidemiology is needed as the size of population covered will depend on a number of different factors including: geography of the area, health and social service boundaries and demography and epidemiology".

In addition, in Wales an audit in 2005 revealed that early intervention services were found to be poorly developed across the country (Welsh Audit Office, 2005). The failure to develop a comprehensive range of services in Wales might highlight a capacity issue where some authorities representing very small 
geographical areas struggle to populate these new teams without impinging on existing provision. The following quote from the Audit Office report supports this view,

"Very few areas have a dedicated early intervention and treatment service or team. A number of CMHTs report that they include early intervention as part of their remit, and in one area a limited number of CMHT staff has been identified and trained to provide an early intervention service" (Welsh Audit Office, 2005 1.39 , p.25).

The evidence shows that specialist EIP teams have proved effective at implementing research evidence, clinical guidelines, and core service features into practice. Bird et al., (2010) showed that effectiveness of EIP implementation may be linked to services being able to deliver interventions recommended by NICE schizophrenia guidance (NICE, 2009, CG 82 \& NICE, 2014, CG178).

What has also become increasingly clear is that a specialist team model is most able to deliver clinical and cost effectiveness. For example, research in Norfolk (Fowler et al., 2009) examined the differences in outcomes between clients provided with CMHT based EIP and those under the care of a comprehensive EIP service. Only $24 \%$ of individuals made a full or partial functional recovery at two years under the CMHT model compared with $52 \%$ of the cases who were under the care of a comprehensive EIP service. A large reduction in inpatient admissions was a further measured benefit of the specialist EIP. Furthermore a systematic review of research evidence (Bird et al., 2011) attributed the effectiveness of EIP to the specialist model of service delivery, recognising its role in enabling the implementation of NICE guidelines and psychological therapies. This evidence informed the National Institute for Clinical Excellence in its 2009 review of the treatment of schizophrenia. NICE favoured the specialist EIP service model as the optimal service configuration system: concluding "Early intervention (for psychosis) can be effective with benefits lasting at least 2 years" (NICE 2009, p29). There are also NICE guidelines for children and young people which puts a much needed emphasis on early recognition and assessment of possible psychotic symptoms (NICE 2013, 
CG155). The updated NICE guidelines for psychosis and schizophrenia for adults aims to improve care through early recognition and treatment which focuses on long-term recovery (NICE, 2014, CG178).

When commenting on a previous model the review went on to say:

"Despite the fact that $\mathrm{CMHT}$ s remain the mainstay of community mental health care (for psychosis), there is surprisingly little evidence to show that they are an effective way of organising services (for psychosis)" (NICE, 2009, CG 82, Section 9.3.4, p336).

The NICE CG178 replaced the previous 2009 title of 'schizophrenia' with 'psychosis and schizophrenia'. Perera and Taylor (2014) critique the CG178 guideline in that the authors use non evidence-based recommendations for instance that the course of CBT should be at least 16 planned sessions. The effectiveness of CBT depends on the skill of the therapist and its fidelity and quality can be difficult to evaluate (Perera \& Taylor, 2014).

\subsection{Policy for Early Intervention}

Mental health policy in England has seen the publication of an array of policy documents and national strategies. The mental health strategies documents have acknowledged the importance of early intervention services (DoH, 1999; DoH, 2000; HM Government, 2009; HM Government, 2011 \& HM Government 2017).

The government's response to the Five Year Forward View for Mental Health (2017) was that children and young people's mental health is a priority in that the government will commit to work with key partners to deliver a five year programme in England. The first access and waiting time standard for people experiencing a first episode of psychosis came into effect in April 2016 which apply to children and young people aged 14 or over.

The Five Year Forward Review (2017) has set out a shared vision on how services need to change in England and what models of care (with emphasises on much more integration) will be needed in the future (Ham \& Murray, 2015). 
The report argues that much more attention should be given to promotion of public health; patients having greater control of their own care; and how barriers in care provided should be broken down. The Five Year Forward View for Mental Health has identified the need to target funding at EIP (Mental Health Taskforce, 2016). The review looks at 'system leadership' and suggests that new kinds of leadership will be needed to make a reality of new models of care. This poses a challenge in that most NHS leaders are foremost organisational leaders rather than system leaders and in turn will have to learn new skills to operate in the NHS of the future (Ham \& Murray, 2015).

The review of the Mental Health Act (2018) says that reducing the numbers of mental health nurses on the NMC register has been declining and services are struggling to recruit staff. Hence, the reduction of the numbers of mental health nurses will make it difficult to deliver services and will prevent the necessary changes to the systems of care needed. The legality of such implications has a profound relevance to delivering services that require expertise, experience and knowledge about the field and potentially could limit developments.

The implementation of early intervention services in England have had concerns that funding for mental health services have disappeared or diluted within non specialist teams. There have been tensions in providing good quality services in the Policy Implementation Guidance (DoH, 2001) and performance targets related to caseloads to continued future funding of services which has a negative effect on the quality of services (Lester et al., 2009). In a study by Radhaknshnan et al., (2017) the authors looked at the costs and the cost effectiveness of degrees of fidelity to the gold standard ( $\mathrm{DoH}, 2001)$. The study examined the cost effectiveness of fidelity levels when using the Department of Health's Policy Implementation Guidance (PIG). The results showed that high fidelity services may not result in improved cost-effectiveness. The high fidelity elements included specialist support from CAMHS, employment interventions with efforts to reduce stigma and improve access and DUP. The analysis showed that the modal level of fidelity was high in terms of the core concept of 
early intervention services; these are dedicated standalone services which operate in an assertive outreach format, with an emphasis on referral and treatment with a skill mix that adheres to those indicated by NICE guidelines. Radhaknshnan et al., (2017) results showed that adhering to fidelity levels of $81-90 \%$ in the PIG was more cost effective than adhering to lower or higher levels. Dropping to a lower level of fidelity may result in wasteful use of resources.

McCrone et al., (2010) has previously established the evidence on the cost effectiveness of early intervention services. Knapp et al., (2013) also showed that individual placement and support which is a NICE approved intervention has been shown to be cost-effective in first episode psychosis which in the longer duration will reduce costs of state support.

Psychological interventions are an important part of care that should be available to service users with a diagnosis of schizophrenia (Haddock, Eisner et al., 2014). The National Institute for Clinical Excellence (NICE, 2014) guideline covers the treatment and management of psychosis and schizophrenia and related disorders in adults (18 years and older) with onset before 60 years. In a study by Haddock, Eisner et al., (2014) aimed to survey mental health services to investigate how many people with a diagnosis of schizophrenia and their families were offered and received CBT or family therapy. The audit of a sample of 187 service users over a 12 month period found that $13(6.9 \%)$ of service users were offered and 10 (5.3\%) received individual CBT, while 3 $(1.6 \%)$ services users were offered and $2(1.1 \%)$ received family interventions within the twelve month audit period. This study reported that the implementation of NICE guidelines for CBT was low, the average number of therapy sessions received fell significantly short of the recommended 16 individual sessions.

The study highlighted barriers to implementation which relate to organisational factors such as lack of time and lack of resources which prevent trained therapists delivering CBT to clients. These findings were in line in Prytys, 
Garety \& Jolley et al., (2011) where lack of resources such as time due to caseloads. Staff attitudes to the effectiveness of psychological therapy were the most prominent barriers to implementation.

In 2015-2016 the Welsh Government invested $£ 800,000$ to develop early intervention in psychosis teams which followed by a further $£ 318,000$ to fund third sector support workers to support with early onset psychosis (Welsh Government, 2018). A strategy was published by the Welsh Assembly Government in 2018 'Together for Mental Health' which aimed to improve mental health and well-being in Wales along with priority areas (Welsh Government, 2018). The strategy acknowledges that while progress has been made across all priority areas that there is always more to achieve to continue to improve and develop services in Wales.

Over the first year the strategy has focused on models of practice which discussed timely access to interventions and has agreed on standardised measures to monitor the impact of interventions. For people accessing psychological therapies a twenty six week wait time target has been set to ensure that people access psychological therapies in a timely way following assessment and progress against the target will be monitored by the Welsh Government (Matrix Cymru, 2017). The document highlights the importance of choice for a range of support for common mental health conditions and individuals with complex needs which is in concordance with the NICE guidelines. There remains however a significant challenge because being in concordance with NICE guidelines is not sufficient implement evidence based practices.

Evidence based Practice (EBP) from a service management perspective is a key quality issue. When a service is operating within an EBP culture both service users and the wider public can be confident that the highest quality and most appropriate clinical services are being provided. Lloyd and King (2009) note that when a service operates outside an EBP culture there is risk that clinical services are outdated, idiosyncratic or simply ineffective. 
It has been previously recognised in England that the majority of healthcare has, in the past, been largely based upon opinion rather than research evidence of clinical effectiveness (Department of Health, 1999). It has been argued that managers and management educators make limited use of the vast behavioural science evidence base relevant to effective organisational practice (Walshe \& Rundall, 2001; Rousseau 2005, 2006a; Pfeffer \& Sutton, 2006). Hodson (2003) found that based on interviews with staff members responsible for promoting the development of EBP in England a combination of 'micro' and 'macro' approaches are most likely to achieve lasting change. Micro approaches focus on changing the attitudes and behaviour of individuals, the assumption being that overall system change will emerge as a result of growing numbers of individuals and groups changing their own ways of working and macro approaches relate to the "top-down" strategy to redesign key systems (such as the dissemination of evidence). Interest in behaviour change interventions targeting health professionals' adoption of clinical guidelines is growing. Existing initiatives to achieve EBP often combine elements of both micro and macro approaches (Nutley \& Davies 1999b). Initiatives that stress education and continuing professional development are at the micro end of the spectrum. Those that focus on issuing guidelines for practice backed up by audit and inspection regimes are more macro in nature.

A critical step towards ensuring the widespread availability of evidence based practices (EBPs) is identifying factors that promote both the initial implementation of EBPs and their long term continuation over time. Sustainability refers to the successful continuation of a program following implementation (Shediac-Rizkallah \& Bone, 1998). Aarons (2004) argues that a better understanding of individual provider attitudes may help to explain staff acceptance of innovation and implementation in mental health settings. There are many examples of attempts to introduce evidence based practice that have failed, even though practitioners accept that recommended approaches are effective and even that they have the skills but nonetheless state, 'I just don't work that way' (King \& Deane, 2009). Statements such as these reflect 
underlying beliefs or attitudes regarding how one practices. For some individuals, these beliefs can be difficult to shift. Yet still little is known about health service provider attitudes towards adoption of EBP's in the British context specifically in community mental health settings.

\subsection{Key Concepts in Organisation Social Context}

The concepts of culture and climate are central constructs in organisational social context (Ashkanasy et al., 2000). The relevance of the organisational social context is important because these are believed to affect implementation of EBPs.

Theory and research suggest the social context of mental health service organisation plays an important role in creating and sustaining shared expectation, attitudes of the clinicians who provide mental health services (Aarons \& Palinkas, 2007; Glisson 2002; Nelson \& Steele, 2007). The adoption of an EBP depends on certain aspects (expectations, perceptions and attitudes). Such as the extent, to which clinicians' are expected to be proficient in their work, perceptions whether clinicians perceive a high level of personal engagement in their work with clients, clinicians' attitudes commitment to the organisation in which they work. These are all believed to encourage or inhibit the adoption of best practices, strengthen or weaken fidelity to establish protocols, support or attenuate positive relationships between service providers and consumers.

Porras and Robinson (1992) and Rousseau (1990) say that one of the most conceptually useful models from the organisational literature for implementation science integrates the social context and technical processes of an organisation to understand how each affect the other. This assumption is particularly relevant to the development of implementation science of the implementation effectiveness in mental health services, because mental health service depends on both social and technical processes and clinicians' expectations, and attitudes can directly affect how consumers are served. 


\subsection{Culture and Climate}

A literature review in the late 1990s found more than 50 definitions of culture and more than 30 definitions of climate within organisations (Verbeke, Volgering \& Hessels, 1998). The literature review helped to address the perplexity around the boundaries that separated the two constructs. Based on a content analysis of the 84 definitions, the review found consensus that culture captured the way things are done in an organisation, and climate captured the way people perceive their work environment (Glisson \& Landsverk et al., 2008).

Culture and climate are related, complex, multidimensional constructs but there is evidence that they are distinct and that each construct affects work attitudes in unique ways (Aarons \& Sawitzky, 2006). Glisson and James (2002) demonstrated that culture and climate are different, but are correlated constructs and thus culture and climate are held to influence attitudes in the workplace. In a more recent review by Jung, Scott and Davies (2009) they found 70 measures of organisational culture, 48 of which had sufficient psychometric information to be assessed. The authors note that culture and climate are often used mutually and that both have been linked to organisational outcomes. In a study by Aarons and Carmazzi (2003) they found that providers working in child and adolescent mental health agencies in the United States with more positive cultures had more positive attitudes towards the adoption of EBP, whereas those with more negative cultures endorsed more negative attitudes towards adoption of EBP.

There are multiple factors at system, organisational and individual levels influencing the implementation of evidence-based practices (EBPs) in mental health care settings (Aarons, Hurlburt \& Horwitz, 2011; Bond, Becker, Drake \& Rapp 2011; Glisson \& Schoenwald, 2005; Bright \& Shadoin, 2008). Any attempt to implement EBP in clinical settings and services needs to consider individual attitudes and organisational culture and climate (King \& Deane, 2009). 
A focus group study by Nelson, Steel and Mize (2006) involving 19 clinicians working in community mental health centres in the USA identified themes around challenges to implementing EBP. The main challenges identified were that most evidence-based practices were too long to be effectively implemented in community practice. They required substantial training to become competent and practitioners believed that research supporting EBP was not applicable to their settings (e.g. trials with restrictive sample characteristics). Practitioners repeatedly indicated that their heavy caseloads did not allow them to make time to learn new approaches and that they did not have the training or supervision needed to implement EBP.

Organisational studies offer a psychological perspective on context. For example Weick (1969) argues that 'external conditions' only become known through the perceptions of organisational members', so that context is fundamentally a mental concept. For Weick (1969:64), 'the human creates the environment to which the [organisational] system adapts'.

A form of this position is taken by writers such as Smircich and Stubbart (1985) and Meek (1988), who see context as wholly enacted through the social construction of actors. For example, organisational culture as a context for action is viewed as an enacted concept and as a result difficult, if not impossible, to change (Meek, 1988)

\subsection{Theory of Planned Behaviour}

The Theory of Planned Behaviour (TPB) contributes to the expectation that attitudes towards adopting an evidence based practice will be related to evidence based practice. In the theory of planned behaviour (Ajzen, 1991) an individual's attitudes regarding a specific behaviour represents an important component in determining whether a specific behaviour will be enacted. The TPB postulates three conceptually independent determinants of intention. The first is the attitude toward the behaviour and refers to the degree to which a person has a favourable or unfavourable evaluation or appraisal of the behaviour in question. The second predictor is a social factor termed subjective 
norm; it refers to the perceived social pressure to perform or not to perform the behaviour. The third precursor of intention is the degree of perceived behavioural control which refers to the perceived ease or difficulty of performing the behaviour and it is assumed to reflect past experience as well as anticipated impediments and obstacles.

The more favourable the attitude and subjective norm with respect to a behaviour, and the greater the perceived behavioural control, the stronger should an individual's intention to perform the behaviour control, the stronger should be an individual's intention to perform the behaviour under consideration.

\subsection{Systems Theory}

Systems thinking emerged within several disciplines (e.g. biology, engineering) in the 1920s. Systems' thinking explores properties that come into existence when several parts have been combined into a whole. It is a way of identifying the inherent organisation within a complex situation and has been called organised complexity. Coiera (2011) argues that the failure of the NHS to introduce system level changes is due to 'system inertia', which is a characteristic of natural systems. 'Systems inertia 'refers to a naturally emergent behaviour, which is aimed at enabling other competing demands to be met when there is a demand for change without the resources of human attention or physical resource. Bertalanffy (1968) distinguished between open and closed systems. Almost all of the systems that are of concern to health and social care professionals are open systems, those that openly exchange with their environment/contexts rather than being closed (entirely autonomous, having no relationship with their environment). It is clearly impossible to treat NHS mental health services as if they are entirely autonomous, given the need for rehabilitation, recovery and community inclusion post-treatment. Coiera (2011) treats the NHS as a bounded organisational system, but in mental health service system terms, this is too narrow a conception. The mental health services system, in order to innovate and change, requires action in more than 
just the NHS. Zokaei et al., (2010) found that the issue of system boundaries was important in that the interfaces between services have a role to play in how the system itself operates.

\subsection{Implementation Science}

Implementation science is defined as the scientific study of methods to promote the systematic uptake of research findings and evidence based practices into routine practice settings (Eccles \& Mittman, 2006). Implementation is the active diffusion-dissemination-implementation continuum. Diffusion is the passive, untargeted and unplanned spread of new practices, dissemination is the active spread of new practices to the target audience using planned strategies, and hence implementation is putting together or integrating new practices within a setting (Greenhalgh, Robert \& Bate, 2004). Of course there are barriers to implementing an evidence based practice which has led to the emergence of implementation and dissemination science. Implementation and dissemination science has its roots in psychological theories. The theory of reasoned action (Ajzen \& Fishbein, 1980), the theory of planned behaviour (Ajzen, 1991), diffusion of innovations theory (Rogers, 2003), and the social cognitive theory (Bandura, 1988; Miller \& Donald, 1941) have all influenced implementation and dissemination science.

In a systematic review by Colquhoun, Squires \& Kolehmainen et al., (2017) they suggest that research identifying the best approaches to implementation do not study interventions in isolation from barriers to practice change, tailored interventions, theory, and stakeholder engagement. The authors indicated that organisational and system level evidence for implementation of best practice remains limited with research on best practice often lacking clarity in the planning and methods.

In a study by Michie, Pilling and Garety et al., (2007) they applied psychological theory to examine possible explanations in implementation. The participants were twenty members of staff comprised of (social workers, nurses, team managers, psychologists and psychiatrists). Semi-structured interviews were 
based on the theoretical framework of behaviour change which addressed the respondents' knowledge, attitudes, and opinions regarding the NICE guideline for schizophrenia family intervention. The authors found differences in professional groups with fewer implementation difficulties among team managers than among nurses and social workers who make more therapeutic decisions about service delivery. Thus, differences between these groups are unlikely to be significant.

\subsection{Fidelity}

Fidelity is defined as the degree to which an intervention was implemented as it was prescribed in the original protocol or as it was intended by the program developers (Dusenbury et al., 2003; Rabin et al., 2008). Fidelity has been measured more often than the other implementation outcomes, typically by comparing the original evidence based intervention and the disseminated / implemented intervention in terms of the adherence to the program protocol, the dose or amount of program delivered and the quality of program delivery. Fidelity is measured through self-report, ratings, direct observations and coding of audio and videotapes of actual encounters, or provider-client/patient interaction.

Fidelity is relevant to my study because I want to examine attitudes towards evidence based practice and how people talk about their experiences of it. 


\subsection{Sustainability}

Sustainability is a key implementation outcome (Proctor et al., 2011) and a priority topic area within implementation science (Glasgow \& Chambers, 2012). Sustained delivery of evidence based interventions is essential to public health impact (Spoth et al., 2011). A critical yet unanswered issue in the field of implementation science is how to conceptualise and evaluate success.

Implementation studies are used widely in varying approaches to measure how well a new mental health treatment, program, or service is implemented. Proctor (2009) defines implementation outcomes as the effects of deliberate and purposive actions to implement new treatments, practices and services. Sustainability is important to the examination of attitudes to evidence based practice because sustainability emphasises the integration of a given program within an organisation culture through policies and practices (particularly the intervention) (Proctor et al., 2011).

\subsection{Implementing Evidence Based Practice in Mental Health}

Implementation of an evidence based guideline in mental health practice is an idea of enormous merit, but this does not always guarantee the acceptance by mental health practitioners (Bilsker \& Goldner, 1999). Despite widespread circulation and publicity of guidelines they are often not implemented that well which leaves a subsequent gap between evidence and practice with health outcomes not achieved (Haines \& Donald, 1998).

Implementation requires greater activity on behalf of researchers and clinicians, but most importantly the systems in which they will work. At a system level the adoption of NICE guidelines has been one implementation strategy, however the existence of guidelines alone does not mean that they are implemented well. 
The Evidence Based Practice Attitudinal Scale (EBPAS) developed by Aarons (2004) assesses four dimensions of attitudes towards adopting EBP, appeal, requirements, openness and divergence. In a sample of 322 clinical and case management mental health service providers for children and adolescent and their families, (Aarons, 2004) found higher educational status, less experience and working in inpatient settings were all associated with more positive attitudes to EBP. In the same sample, the association of organisational culture and climate on EBP attitudes was also explored (Aarons \& Sawitzky, 2006). It was found that workers in organisations considered to have more constructive cultures and workers earlier in their professional careers had more positive attitudes towards adopting EBP. The authors argued that, 'having a positively perceived local opinion leader who can influence organisational culture who can introduce and guide change in practice may facilitate receptivity to change in provider behaviour' (Aarons \& Sawitzky, 2006. p .68).

\subsection{Clinician Attitudes towards EBP}

Efforts to determine effective ways for disseminating and implementing interventions are supported by the results of rigorous research called evidence based practice (EBP). These efforts have included the examination of clinician attitudes towards EBP (Aarons, 2004) and the impact of organisational culture (Glisson et al., 2008). The role of attitudes and acceptance of innovation literature around evidence based practices has mainly been conducted in the United States within adolescent and children's mental health.

Attitudes can be a precursor to the decision of whether or not to try a new practice and the affective component of attitudes can impact decision processes regarding innovation (Frambach \& Schillewaert, 2002). In a study of 322 clinical and case management mental health service providers for children, adolescents and their families, Aarons (2004) found higher educational status, less experience and working in inpatient settings were all associated with more positive attitudes towards EBP. In a study by Jensen-Doss, Hawley, Lopez and Osterberg (2009) surveyed community clinicians in the US who were working 
under a mandate by the state to implement EBP. They found that the clinicians on average held slightly negative views of EBP and believed that their colleagues did as well. Interestingly clinicians with more positive attitudes towards EBP reported using the interventions more often. Proctor, Knudsen and Fedoravicius (2007) say that clinicians' attitudes toward EBP are influenced by the perceived fit between EBP within the organisation, with re-invention often needed.

In a study by Allen and Armstrong (2014) in a sample of 255 clinicians from the Treatment Attitudes, Perceptions and Practices for Neglected and Abused Children project found that the clinicians would use an intervention given various types of evidence. The most preferred type of evidence with the most positive attitudes towards evidence based practices predicted preferences for clinical trials. Clinicians preferred case studies regardless of the attitude towards evidence based practice. Case studies as a dissemination method may increase the implementation of evidence based practice.

In a study by Michie, Pilling, Garety et al., (2007) they suggested the importance of systemic influence and organisational attitude in the UK was highlighted in a study aiming to identify the barriers of family intervention in schizophrenia. The study showed resources such as time and training to be the biggest barriers to adoption.

This evidence suggests a number of issues. The way in which practitioners view knowledge is an issue as each discipline has different traditions of empirical research in community services and each draws differently on qualitative and quantitative methods and underlying theories of knowledge. The second issue is the process of implementation involves not only increasing awareness of research findings, but integrating them into routine clinical practice. The adoption of clinical guidelines such as the National Institute for Clinical Excellence (NICE) used in the UK have been implemented at a system level, however the existence of these guidelines alone does not mean that they are implemented. This study looks at early intervention practitioner's attitudes towards adopting EBPs in England and Wales in early intervention in psychosis 
teams and what they say about their experiences of adopting evidence in their practices.

\subsection{Research Questions}

This study set out to explore how evidence based practices were implemented and sustained in mental health services. In particular the focus was on early intervention services in England and Wales. The approach used was an explanatory sequential mixed method study to answer the following research questions;

1. What are the attitudes of early intervention lead practitioners in relation to evidence based practice?

2. To what extent is service context and culture relevant to practitioner's experiences of adopting evidence based practice?

3. What do early intervention lead practitioners say are the crucial factors in sustaining or not sustaining practices in their teams in England and Wales?

4. What do early intervention lead practitioners say are the facilitators and barriers to implementation of evidence based practices in their services?

\subsection{Rationale for this Study}

If the most efficacious and effective interventions are to be disseminated and implemented in community-based settings, a better understanding of attitudes of providers is needed in order to more effectively tailor dissemination and implementation efforts in relation to provider individual differences in the service context. The study reported here is a response to the call for a better understanding of the context into which evidence-based practices (EBPs) are likely to be disseminated (e.g., Burns, Hoagwood, \& Mrazek, 1999; Glisson, 
2002; Hoagwood, Burns, Kiser, Ringeisen, \& Schoenwald, 2001; Schoenwald \& Hoagwood, 2001).

\subsection{The Researcher}

My background is in health science with a masters degree in research methods - psychology. My interest in this area was prompted when working as a research assistant on a NIHR Service Delivery and Organisation funded research programme. That study looked at integration of social care within multidisciplinary community mental health teams (CMHTs) in England and Wales (Huxley et al.,2011). My particular interest was drawn to team culture, climate, in particular team composition. Interest naturally followed toward the adherence to policies (fidelity) and how these may or may not be implemented by senior managers within a clinical role. This work showed different professional backgrounds implemented evidence based practices (particularly supportive treatments) in community mental health services to varying degrees. There were marked differences in the ways that professionals of different disciplines, (health, social work and generic) implemented policies. Fortunately for me, this work led to two publications (Huxley \& White et al., 2011 \& 2012). Interest in schizophrenia and psychosis came about when I was appointed to my second research assistant position. I worked on the DUETs, the UK database of "uncertainties about the effects of treatments", this is part of the NHS evidence database (Fenton, Timimi \& Chalmers, 2006). This work was brought together jointly by the, James Lind Alliance (JLA) and DUETs. The aim was to bring the philosophy and practice much closer in such a way that; patient, carers and clinicians may rank questions about the effect of treatment/s for a given disease. The priority setting partnership was formed following an exercise by Swansea University to identify uncertainties from patients, carers and clinicians regarding treatment for schizophrenia. This work led to two publications in the journal Nature (Lloyd \& White, 2011; \& 2012). My role working as Research Portfolio Development Fellow on the Mental Health Research Network Cymru project further enriched my interest and knowledge in early intervention and implementation of these teams in England and Wales. My 
particular interest in these teams stems from previous research. It suggests that services can have better outcomes when delivered by multidisciplinary teams, and, that new teams may have even better outcomes than CMHT's.

Subsequently, I have taken a particular interest in the scientific study method that can promote, to some degree, the uptake of research findings into healthcare from a clinical organisational perspective. Throughout my PhD journey, I have become interested in how services in mental health are commissioned and the role of the hybrid manager (clinical professionals turned managers) in a clinical setting.

\subsection{The Study}

The approach I have adopted in this study has been to explore the attitudes and experiences of implementation of evidence based practices in mental health with early intervention practitioners. My interest was in exploring practitioners' attitudes and within different professional groups. I became interested how EBPs are implemented within clinical setting. This interest then led on to considering the concept of fidelity which refers to the degree to which an intervention was implemented as it was prescribed in the original protocol or as it was intended by the program developers.

My study is an explanatory sequential mixed method design which consisted of two phases; quantitative data collection and analysis followed by qualitative data collection (Creswell, Plano Clark, et al., 2003).

In addressing attitudes and adoption to an EBP I first administered Aarons (2004) Evidence Based Practice Attitudinal Scale (EBPAS) which represented four distinct constructs involving willingness to adopt EBPs given their intuitive appeal, willingness to adopt new practices if required, general openness toward new or innovative practices, and perceived divergence of usual practice with academically developed or research based practices. 
The second component of the study was the qualitative research interviews with 18 practitioners working in NHS trusts in England and Wales. The semistructured interviews looked at every day experiences of implementation issues in early intervention teams in relation to sustainability, fidelity, barriers and facilitators of evidence based practices in the practitioners' organisations. A mixed methods approach has enabled me to address my research questions. I was interested in the question 'what works' which is a pragmatist worldview for mixed methods (Cherryholmes, 1992). Pragmatism advances multiple pluralistic approaches to knowing using the 'what works' question.

\subsection{Structure of the Thesis}

The thesis is structured into seven chapters and these describe how the study has been undertaken.

- In the current I have provided a general introduction to the research topic, the research and the approach adopted in this thesis.

- In Chapter two I review a range of research evidence and use this to show the effectiveness of early intervention teams. The studies reviewed are early intervention services, cognitive behavioural therapy (CBT), and family intervention in early psychosis and two Cochrane Systematic Reviews of family intervention and Early Intervention in Psychosis.

- Chapter three describes the methods used in the study. It provides the methodological approach undertaken and explains why a mixed methods study was undertaken. The research design is described including the approach that was undertaken to data collection and analysis. Ethical issues arising from the study are also discussed.

- Chapter four presents the findings from the quantitative phases of the study. In the first section the results of the online survey which was undertaken with early intervention practitioners working in NHS trusts are reported. In this chapter I demonstrate via my analysis that openness and requirement subscales were good predictors of attitudes. 
- In chapter five I show via my analysis of interview talk of lead practitioners in early intervention services that they are concerned with sustaining evidence based practices. They also report challenges and opportunities to adopting evidence based practices which focuses on the barriers and protection of early intervention services.

- Chapter six presents the discussion of quantitative component findings along with qualitative discussion and then the integration of both components via the mixed methods matrix.

- Chapter seven presents the conclusion in which I lay out original contribution of this research and suggest a number of implications for future research in relation to evidence implementation. 


\section{CHAPTER 2: Literature Review}

\subsection{Introduction}

In this chapter I present a narrative review of the literature in regard to early intervention evidence based practices within mental health, particularly, early intervention teams in England and Wales and how people experience the delivery of these services. This chapter is structured in three parts. In the first part I examine the policy for early intervention which is largely specific to England. I follow this by considering the evidence base for the effectiveness of early intervention services including literature researching family therapy and cognitive behavioural therapy. In the second part of the chapter I review key concepts in organisational social context such as culture and climate and theories such as the theory of planned behaviour and social systems theory. In the third and final part I examine the definition of evidence based practice and how this can be conceptualised by researchers and practitioners.

\subsection{Rationale for Type of Review}

I originally anticipated conducting a systematic review via the Effective Practice and Organisation of Care (EPOC) Cochrane group. Systematic reviews however can become too narrow and limit the use of evidence arising from different types of studies, both qualitative and quantitative. Prior to beginning the review, searches were conducted in an attempt to identify previous systematic reviews on the topic of evidence based practice. One intervention review was published namely, 'the effectiveness of organisation infrastructure to promote evidence-based nursing practice' by Flodgren et al., (2011). The Flodgren et al., (2011) review was part of my decision making process deciding to conduct a narrative review. The Flodgren et al., (2011) found that many studies were ineligible for inclusion in their systematic review due to poor design and so I took the decision to use the more permissive inclusion approach allowed by a narrative review that would enable a greater range of research studies to be considered. 
With EPOC systematic reviews authors frequently experience the issue of 'lumping and splitting' (Gøtzsche, 2000). This is where the focus of a review is too narrow or too broad. Narrative reviews can offer a scholarly summary along with interpretation and critique (Greenhalgh, Thorne \& Malterud, 2018). Narrative reviews are aimed at identifying and summarizing what has been previously published, avoiding duplications and seeking new study areas not yet addressed (Ferrari, 2015).

\subsubsection{Decision making process around which studies were included}

In reading the papers I found that most of the research conducted on mental health and clinician attitudes has been done in the United States particularly focusing on children and adolescents.

The decision making process around the types of studies that I was interested in were those that generated empirical evidence on EIP which included quantitative studies. I only included UK studies for early intervention in psychosis particularly comparing NICE EIP to standard care. I used the PICO framework (Patient, Intervention, Comparison and Outcome) by Richardson, Wilson, Nishikawa and Hayward (1995) which allowed me to take an evidence based approach to my literature searching which enabled me to frame the type of studies to include and exclude. I did not use an appraisal approach as such but I judged the papers on sample size and methodological limitations.

I searched for and included studies of practitioner attitudes to evidence based practice and studies examining professional organisational culture and climate.

\subsubsection{Types of Studies (Eligibility Criteria)}

Studies where implementation of an intervention intended to improve practice and performance in the use of EBP were considered. Interventions which will make a change at an organisational level to improve healthcare performance 
were sought. Interventions could be CBT, family interventions, assertive outreach team, and early intervention teams.

Types of studies considered for the narrative review: Randomised Control Trials (RCTs), (example citations of RCT's that met the criteria are: Optimal Treatment Project (OTP) trial by Grawe, Falloon and Skogvoll (2006); CBT for psychosis single blind RCT by Lecomte et al., 2008 and an RCT of family intervention by Pharoah et al., (2010).

\subsection{Review Method}

Below I describe the process I undertook in searching for articles which included the search strategy, screening, and selection of studies according to my eligibility criteria.

\subsubsection{Searching}

Articles presented in this search strategy were identified and the search strategy developed with the help of an experienced librarian and was conducted through searches of databases including MEDLINE, PubMed, CINAHL, PsychARTICLES and PsychINFO via the EBSCO platform using key terms in the English language from 2007 to 2018. Grey literature was searched via theses collection for mixed methods and mental health theses. The search strategy is presented in Appendix 1.

I conducted my searches of these databases as three separate searches and finally as a combined search as follows:

Search one included the following terms: implementation-of-change OR implement* change OR change-management OR managing-change OR organisational-change OR organizational-change OR diffusion-of-innovation OR "dissemination and implementation".

Search two included: evidence-based OR evidence-informed OR researchbased OR research-informed OR EBP OR EBM OR clinical guidance OR clinical guidelines OR practice guidelines.

Search three included: mental OR psychiatric and search four combined searches one, two and three. 
Limits for the searches were as follows: papers in the English language, dates were from 2007 to 2018 and peer reviewed journal articles only were selected. The searches were conducted in 2014 and last updated in November 2018.

From the original search 348 articles were returned from the combined three searches. Visual inspection of the titles of papers resulted in 267 being excluded as they were clearly not relevant to the topic. A few examples of the type of papers excluded were that papers focused on quality assurance, nursing care standards, informatics, health promotion methods and primary care. There were 81 full text articles remaining and these were further assessed for eligibility. Upon closer inspection 45 papers were excluded with reasons such as they were commentary or discussion papers, social work practice papers, studies focusing on child and family youth mental health, substance abuse services, paediatric acute care, children's mental health treatment, USA Assertive community treatment, substance use treatment, medication management, dialectical behaviour therapy, medication management, case management, crisis management, family support, autism, psychotherapists, paediatrics, nursing, primary healthcare and military veterans.

I engaged in back-chaining by searching for references in papers located and then locating those papers to see if they were relevant.

I searched for and included studies of practitioner attitudes to evidence based practice and studies examining professional organisational culture and climate. This approach enabled me to focus on addressing the first two research questions to understand what attitudes to evidenced based practice had already been researched and any lessons arising from this work and the role of context and culture in adopting evidenced based practice.

A total of 11 articles were retained for review and obtained by the researcher. In re-running the literature searches to include new material from the combined three searches set out above 38 papers were identified which were relevant. Of these 38 papers, two papers were retained which focused on early intervention in psychosis. The two papers namely Stein, Common, Pilton et al., (2019) and 
Adamson, Barrass, Mcconville et al., (2018), were not included with the 11 papers as they were not adding anything new.

In reading and screening the papers I found that most of the research conducted on mental health and clinician attitudes has been done in the United States particularly focusing on children and adolescents.

The decision making process around the types of studies that I was interested in were those that generated empirical evidence on EIP which included quantitative studies. I only included UK studies for early intervention in psychosis particularly comparing NICE EIP to standard care. I made this decision because I wanted to examine evidence of direct relevance to the organisational context of UK mental health services.

The literature was themed as theme titles which are: the concept of early intervention in psychosis, service model options and evidence based practices. 


\subsection{The Concept of Early Intervention in Psychosis (EIP)}

Early intervention in Psychosis (EIP) is an evidence-based approach aimed at detection and treatment of psychotic symptoms in their early stages. The aim of early intervention is to reduce the long-term adverse impact of psychosis with the aim of preventing relapse (Csillag, Nordentoft, Masafumi et al., 2016). There are two elements in relation to early intervention. The first is early detection within the prodromal period (which is the disruption phase prior to the onset of psychosis). The second element is the delivery of early intervention services that bring intensive treatment services through multidisciplinary teams with a view to reducing duration of untreated psychosis (DUP). DUP is an important determinant of clinical and functional outcome (Marshal et al., 2005; Perkins et al., 2005). Pathways to care for patients with first episode psychosis (FEP) are vital for informing the provision of early intervention services, which focuses on shortening DUP and ensuring care during the initial stages of illness (McGorry et al., 2007).

\subsection{The Effectiveness of Early Intervention Teams}

There has been numerous debates and speculation upon the effectiveness of early intervention services. Below I present the evidence of the effectiveness of early intervention in terms of the evidence base. The services provided include early intervention services, interventions such as Cognitive Behavioural Therapy (CBT) and family intervention.

\subsubsection{Early Intervention Services}

The development of early intervention services for young people with firstepisode psychosis has become a priority in a number of countries including England, Canada, Denmark, Australia and Scandinavia (McDaid et al., 2016). England was the first adopter with early services piloted in the West Midlands in the 1990s (Lester et al., 2009 \& Marshall et al., 2014). Evidence from the West Midlands early intervention teams, increased campaigning, evaluative evidence from Australia, coupled with an increased focus on mental health with a change 
of government in 1997 all acted as an acceleration for the substantial investment in early intervention teams that was to follow.

There has been an ongoing debate as to whether Early Intervention in Psychosis (EIP) has been a waste of valuable resources (Pelosi \& Birchwood, 2003). Two randomised controlled trials namely, the Lambeth Early Onset trial and OPUS and several effectiveness studies of routine early intervention services show that specialised teams are superior to generic care in managing the critical phase of psychosis (Craig et al., 2004; Garety et al., 2006; Nordentoft, Rasmussen, Melau, Hjorthøj \& Thorup, 2015; Petersen et al., 2005 \& Singh, 2010).

In the section that follows I discuss the research evidence which includes randomised controlled trials (RCTs) for delivery for early intervention. In the Croydon Outreach and Assertive Support Team (COAST) study by Kuipers, Holloway, Rabe-Hesketh \& Tennakoon, (2004) a randomised controlled trial (RCT) was used to look at the effectiveness for its evidence base during its first year. Fifty-nine participants were randomised to COAST or treatment as usual (TAU). The interventions offered were atypical medication psychological interventions and a range of vocational help according to need. The outcomes were evaluated at baseline, 6 months and 9 months on a range of standardised clinical and social measures. Overall COAST and TAU clients improved over time, but there were no statistically significant improvements for COAST clients, bed days were less in COAST, though this was not significant. The research showed that both groups improved in symptoms and functioning, the study suggests that community mental health teams should offer high quality input at any stage of psychosis in order to meet the needs of clients and carers.

The Lambeth Early Onset (LEO) study evaluated the effectiveness of an Early Intervention in Psychosis service compliant with the 2001 DoH Policy Implementation Guidance (Grawe et al., 2006). The participants were 144 
people aged 16 to 40 years presenting to mental health services for the first or second time with non-affective psychosis. The study found that a specialist EIP team delivering care and treatment for young people with early psychosis was superior to standard care (defined as a traditional CMHT encouraged to follow EIP principles) for maintaining contact with services and reducing readmissions to hospital (Craig et al., 2004). The study did have limitations in that it was acknowledged that the sample was underpowered. The authors relied on record systems for data on relapse which introduces the possibility of risk of errors and biases. The authors indicate that they used an apriori operational definition of recovery and relapse with clear definitions and a rating manual to aid standardisation of assessments.

The OPUS study has been subjected to clinical and economic evaluation (Petersen et al., 2005). The study was conducted in Denmark and included more than 500 participants (aged 18 to 45 years and a legal residence). It evaluated the impact of a modified assertive community treatment integrated programme compared with standard treatment for people with first episode psychosis. In the assertive community treatment arm of the study the caseload did not exceed 10:1, the cornerstone of the treatment was supportive and outreaching contact. In the standard treatment arm of the study, the patient was offered mental health treatment at a centre. The caseload of the staff in the centres ranges from 20 to 30 people per staff member. Most patients were seen in office premises with contact by either a physician, CPN or a social worker. Social skills training was not offered in the community mental health centres. The antipsychotic medication offered was based on the same principles for both arms of the study. The study showed clinical benefits after one year of a flexible needs-based programme which was greater than standard treatment. The treatment focused on home based assertive case management integrated with pharmacotherapy, with family or individual psycho-education, with social problem-solving skills training offered when indicated. 
In an Optimal Treatment Project (OTP) trial by Grawe, Falloon and Skogvoll (2006) they randomised patients into two groups the first was standard treatment and the second was integrated treatment (patients being treated via a multi-disciplinary team). The standard treatment consisted of patients receiving regular clinic-based case management with antipsychotic drugs, supportive housing and daycare, crisis and in-patient treatment at psychiatric hospitals. Rehabilitation which promotes independent living, a brief psycho educational and supportive psychotherapy were also provided. Eighty percent of the patients received standard treatment from out of hospital out-patient services and the remainder from local community general health services. In the integrated treatment arm of the study the patients were treated by a multidisciplinary team which was independent of the standard treatment programme. Pharmacotherapy and case management was similar to standard treatment with a low case load (patient-staff ratio approximately 1:10). The integrated treatment group was superior to standard treatment group. The composite index showed that significantly more clinical recovery patients (53\%) had excellent two year outcomes than the standard treatment group (25\%). Integrated treatment reduced negative symptoms, minor psychotic episodes and in stabilising positive symptoms but did not reduce hospital admissions or major psychotic recurrences.

In a Cochrane review of early intervention for psychosis by Marshall and Rathbone (2011) the authors conducted systematic review of randomised controlled trials aimed at preventing progression of psychosis in people showing prodromal symptoms or early stages of schizophrenia. The study aimed to evaluate the effects of early detection, phase-specific treatments (psychological, social or physical treatment) and specialised early intervention teams. These results suggest some support for specialised early intervention services but more trials would be required to see if gains were maintained. The authors suggest that there was some support for phase specific treatment focused on employment and family therapy, but again, more trials were required. 


\subsubsection{Cognitive Behavioural Therapy}

The cognitive orientated psychotherapy for early psychosis (COPE) study (Jackson et al., 2005) aimed to facilitate the adjustment of the person and prevent secondary morbidity in the wake of a first psychotic episode. The study reported on outcomes of a controlled trial comparing two conditions: COPE versus No-COPE. Ninety-one people participated in the trial which analysed intention to treat, including 12 people who were assigned to COPE but refused to participate. The assessments were conducted at pre-treatment, midtreatment and post treatment. The study was conducted in a public mental health service, the Early Psychosis Prevention and Intervention Centre (EPPIC). The COPE consisted of four phases: engagement, assessment, adaptation and secondary morbidity. The therapy was manualised and engagement sessions ranged from three to four sessions. The study showed that there were no significant differences found between the two groups. The authors say that most people were readmitted over $50 \%$ in the COPE group and nearly $44 \%$ in the No-COPE group, with most readmissions occurring in the first year during the treatment period. There were a number of limitations with the COPE study. The authors did not measure non-compliance with medication and actual medication doses were not measured over the course of the trial, but rather in a static way at baseline. The protocol for the study did not fix the number, duration or frequency or order of sessions. Not all patients received all the components of the treatment. This study suggests that COPE does not present any advantage to those patients receiving therapy over those who are not received therapy.

Lecomte et al., (2008) evaluated the effectiveness of CBT for psychosis by conducting a single blind randomized controlled trial. They compared manualised group CBT intervention for first episode to a manualised group social skills intervention for symptom management. The workers delivering the CBT for psychosis were not qualified CBT therapists. They performed satisfactorily in the delivery of group therapy with the help of a structured manual, on most therapeutic aspects measured. The results suggest that qualified $\mathrm{CBT}$ therapists are not needed, but rather training mental health 
workers in delivering a group CBT intervention may be sufficient. The authors note that a group approach to delivering CBT might be an alternative to waiting for more funds and more highly qualified people to be trained. This is an interesting finding as the authors say that both interventions were delivered by mental health staff with minimal training.

\subsubsection{Family Intervention}

Family intervention is an effective intervention for people with schizophrenia. (Pilling et al., 2002). The UK clinical guideline recommends that family intervention should be offered to those families of people with schizophrenia who have relapsed or who are considered at risk of relapse (NICE, 2014). Despite the guideline, the intervention is underused (Magliano et al., 2005).

Leavey et al., (2004) conducted a randomised controlled trial of a brief intervention for families of patients with first episode psychosis. Carers of patients with first episode psychosis in North London were invited to take part. The intervention comprised of education and advice about the condition from a support team or usual care from community psychiatric services. The study found that the support and advice for families had little impact on their ratings of satisfaction and patient's outcomes. Uptake of the intervention was poor and hence this was reflected in the power effect size which was reduced. The authors say that providing support and educational intervention shortly after first onset may be difficult particularly if patient's symptoms have gone. Carers would often inform support workers that continued association with psychiatric services was perceived as both a painful reminder and unnecessary. The point of providing information and advice to carers of people newly diagnosed required further study.

Zhang, Wang, Li and Phillips (1994) conducted a randomised control trial of family intervention for 78 first episode men with a diagnosis of schizophrenia in China. The patients were assigned to family intervention or standard care. The results showed that there was significantly lower rate of hospital readmission in 
the family intervention group than the control group. This intervention therefore shows cross cultural effectiveness.

In a research paper by Ince, Tai and Haddock (2016) they looked at behavioural specific plain English in psychological intervention guidance to improve healthcare intentions in line with guidance for schizophrenia. Participants were randomly assigned to either the original text or the alternative behaviourally specific, plain English version. The authors found that writing psychological intervention guidance recommendations for schizophrenia is not sufficient to significantly improve the cognitive determinants towards action as defined by the theory of planned behaviour in CMHT health care staff.

A review of family intervention for schizophrenia by Pharoah et al., (2010) selected randomised and quasi-randomised studies focusing primarily on families of people with schizophrenia that compared community family based psychosocial intervention with standard care. Qualitative reviews highlight the possible advantages of using family interventions for those with serious mental illnesses (e.g. Leff, 1995). Quantitative reviews are less common (e.g. Mari, 1994). This updated review adds 21 additional studies, with a total of 53 randomised controlled trials included. The results showed that family intervention decreased the frequency of relapse. The authors report that quality of the reporting was poor. Only eight studies from 53 described the method of randomisation and one study described the method of allocation concealment.

\subsection{Summary}

In the previous sections I have reported on nine trials from early intervention services. These were; COAST (Kuipers, et al., 2004), LEO (Craig et al., 2004), OPUS (Petersen, 2005), OTP (Grawe et al., 2006) and two Cochrane reviews one by Marshall and Rathbone (2011) in Early Intervention in Psychosis and the second Cochrane review in family intervention for schizophrenia by Pharoah et al., (2011). 
The COAST trial (Kuipers, et al., 2004) showed clients in COAST and TAU improved over time, disappointingly there was no significant improvements for COAST clients. There was a trend for COAST carers' quality of life to increase. Bed days were also less in COAST, but not significantly so. The concluding remark from this study was that CMHT's should aim to offer high quality input at any stage of psychosis in order to meet client and carer needs.

Research conducted by the Lambeth Early Onset service (LEO) Craig et al., 2004) clearly indicate that Early Intervention in Psychosis (EIP) delivers better outcomes than the standard CMHT. The EIP clients were more often in regular contact with the clinical team, and were more likely to attend appointments.

Clients were more likely to have been offered psychosocial interventions and to be in recovery, they also had fewer admissions and better social and vocational functioning. Gafoor (2010) highlights that continuing the EIP approach for some clients beyond the three years is important as the LEO outcomes did show some loss of earlier gains.

The Danish OPUS study (Petersen, 2005) demonstrated a beneficial effect after 2 years. The intervention group had a significantly lower level of psychotic and negative symptoms, fewer in-patient days, better treatment adherence and higher level of service user satisfaction. The OTP by (Grawe et al., 2006) study showed that evidence-based treatment achieves greater clinical benefits than pharmacotherapy and case management alone for recent-onset schizophrenia. For the CBT there were two studies for CBT, COPE (Jackson et al., 2005) and (Lecomte et al., 2008).

For the CBT studies COPE (Jackson et al., 2005) there was no evidence that COPE added to the range of Early Psychosis Prevention and Intervention Centre (EPPIC). The authors' state that the study effect of providing more sessions and more active session. The study suggests that assessing the ability of therapist to engage patients in active therapy. In a study by Lecomte et al., (2008) the CBT for psychosis as well as the symptom management skills training offer specific advantages over treatment as usual in terms of psychosis symptom reduction for individuals with recent-onset psychosis. 
For family intervention there were four studies (Pilling et al., 2002), (Leavey et al., 2004), (Zhang et al., 1994), (Ince et al., 2016). For the family intervention study (Pilling et al., 2002) single family therapy had clear preventative effects on the outcomes of psychotic relapse and readmission, in addition to medication compliance. The CBT improved mental state and demonstrated positive effects of follow up.

In the study by Leavey et al., (2004) they found that the support and advice had little effect on satisfaction or on patients outcomes. This might have been the power to show an effect was reduced in that families did not take up, or dropped out of the intervention which threatened the internal validity of the trial which might have raised the possibility of a type 2 error. Authors state that providing education support shortly after onset may be difficult. However family intervention in general is considered important and is liked by carers. In the study by Zhang et al., (1994) the majority of sessions did not include the patient. There was significantly lower hospital readmission in the family intervention group than the control group. In the study by Ince et al., (2016) they conducted a systematic review on psychological interventions for schizophrenia. The rates of implementation for CBT and family intervention were compared. They found that rates of implementation for $\mathrm{CBT}$ and family intervention were below the recommended levels with wide variation rates found. Practitioners observations were: inequalities in the provision of psychological therapies for schizophrenia persist, CBT and family intervention do not ensure implantation and collaboration at all levels of healthcare is need for effective implementation. 


\subsection{Service Model Options}

There are several service delivery models that can be described as potential drivers for delivering key components of Early Intervention in Psychosis (EIP). These are (1) traditional or enhanced CMHT model (sometimes described as a 'dispersed model') (2) hub and spoke model (3) specialist team model.

Below I describe the models and the analysis of the strengths and weaknesses in terms of their ability to achieve the key tasks and delivery of components of early intervention.

Many of the clinical interventions require delivering an effective service to EIP service users and their families and it appears that these are easier to deliver from a specialist team (Dodgson \& McGowan, 2010). The strongest evidence base is also for this model type (Bird et al., 2010). In some parts of England, this model appears to be compromised due to the geography of the area or other service based factors such as area square miles and expected incidence rates along with population. If a specialist team is not possible, then any compromise should have a mechanism for ensuring fidelity to the EIP model and the new ways of working. Dispersed models such as the CMHT model do not have this mechanism, which can make them appear that there may be no need to fund a manager or specialist staff member such as psychiatrists. Dodgson \& McGowan (2010) have argued that even so, they are likely to be less effective and the roles of manager and psychiatrist will still be required, but will be provided by non-specialist workers. Therefore any potential financial saving is theoretical not real.

\subsubsection{Traditional Community Mental Health Team (CMHT) Model}

The traditional CMHT model can include enhanced Community Mental Health Team (CMHT) model which is sometimes described as a dispersed model. The CMHT model is designed to be multidisciplinary with a skill mix and that offers a range of bio-psycho-social interventions to clients with a range of mental health problems (Huxley \& White et al., 2011). The CMHT model as the 
standard model in England has been used as the condition for 'standard care' in empirical studies.

\subsubsection{Hub-and-Spoke Model}

The service is provided by staff who are to be embedded in 'spokes', often generic CMHTs, and in the central 'hub'. The hub usually provides access to leadership, specialist skills and support to the spoke workers. This model is often found in rural areas. There are risks involved with this model such as isolation of EIP workers, limited supervision time, lack of availability of competent therapists and gradual increase in caseload numbers (Singh \& Fisher, 2007).

\subsubsection{Specialist Model}

This model involves staff being mainly or exclusively responsible for the care and treatment of young people with early psychosis within a discrete, specialist, free standing team. The specialist service model has been supported by Marshall et al., (2005) which meticulously follows the recommendations for service management in the Policy Implementation Guide ( $\mathrm{DoH}, 2001)$. The staff are expected to follow the core principles of care, but often have limited contact with people in similar roles. Dodgson \& McGowan (2010) say that this is the least expensive model to implement. Analysis of service costs associated with specialist service teams compared to standard care have been demonstrated to be cost effective (McCrone \& Knapp, 2013).

The specialised model prescribed in the policy implementation guideline will not be appropriate in all localities. In rural areas for example the geographical spread is different, and hence the clients will determine the nature and intensity of the early intervention service. The incidence and prevalence of psychosis also has an impinging factor on the development of standalone services. The specialist service model has been supported as a model of choice by Marshall et al., (2004). Evidence from randomised controlled studies (Craig et al., 2004; Kuipers et al., 2004; Petersen et al., 2005 and Graawe et al., 2006) indicates that the approach to delivering EIP is highly effective. Specialist 
teams are highly valued by service users and carers (Lester \& Birchwood, 2009) and they produce better clinical outcomes than the traditional CMHT (Singh, 2010). Friis (2010) notes that specialist services invest in high levels of engagement with patients which is not possible in the traditional CMHT.

\subsection{Evidence-Based Practices}

Evidence-based practices (EBPs) are interventions for which there is consistent scientific evidence showing that they improve client outcomes (Drake, 2009). The most famous and quoted definition of EBPs is Sackett et al., (1996) definition below.

Sackett et al., (1996) define evidence-based practice as:

"The conscientious, judicious use of current best evidence in making decisions about the care of individual patients. The practice of evidence based medicine means integrating individual clinical expertise with the best available external clinical evidence from systematic research." (p.1).

Over the last fifteen years, there has been a big change in the way that health care professionals use evidence from scientific research in their day to day working practices (clinical practices). Sackett and Rosenberg's (1995) concept of evidence based health care has become part of the language of clinicians, managers, policymakers and researchers in health services throughout the world.

The American Psychological Association (APA, 2005) adopted a policy statement:

'Evidence-based practice in psychology (EBPP) is the integration of the best available research with clinical expertise in the context of patient characteristics, culture and preferences'. (p.173).

This is broadly consistent with a widely quoted definition applied by Sackett et al., (1997, p.2) definition of evidence-based medicine: 'the conscientious, explicit, and judicious use of current best evidence in making decisions about the care of the individual patients'. 
The APA definition arose out of a period of highly contentious debate within the profession (APA Presidential Task Force on Evidence Based Practice, 2006). One way of understanding this debate is to view it as a debate between researchers and practitioners. Researchers attach high levels of importance to the results of clinical trials and tend to view that clinical outcomes will be best when practitioners conduct their treatments in the manner of a clinical trial. By contrast practitioners are suspicious of clinical trials and fear that clinical judgement will be replaced by a straightjacket of highly standardised treatment protocols. The consensus definition like Sackett et al., 1997 definition is an attempt to accommodate the values and concerns of both groups.

The term evidence based practice assumes that it is practical and desirable to base practice on knowledge of what works. This urges the question of how knowledge is generated, validated, disseminated and adopted (Nutley \& Davies, 2000) and more crucially who is involved in each of these activities. A traditional model of evidence based practice is presented below in figure 1. In the model there are four stages in the linear process which starts with knowledge creation and ends with adoption. The process involves two main communities of experts (researchers based in Universities) and users (practitioners based in the field). There is limited interaction between these two communities. The model has been challenged on the account of linearity and the separate domains occupied by University researchers and practitioners (Gibbons et al., 1994).

An alternative conceptualisation is presented in figure 2 below noting the sharp boundaries between knowledge production and utilisation. Gibbons et al., (1994) tells us that this assumption is of a continuous interaction between knowledge creation, validation, dissemination and adoption stating that none of these activities belongs to the separate domain of experts as opposed to users. Instead both experts and users are partners in the generation and utilisation of knowledge. Gibbons (1994) refers to this as knowledge creation though applied partnerships. 
Figure 1: Mode 1 - traditional model of evidence based practice adapted from Nutley and Davies (2000) p 325.

RESEARCH EXPERTS

(evidence generators)

\section{PRACTITIONERS}

(research users)

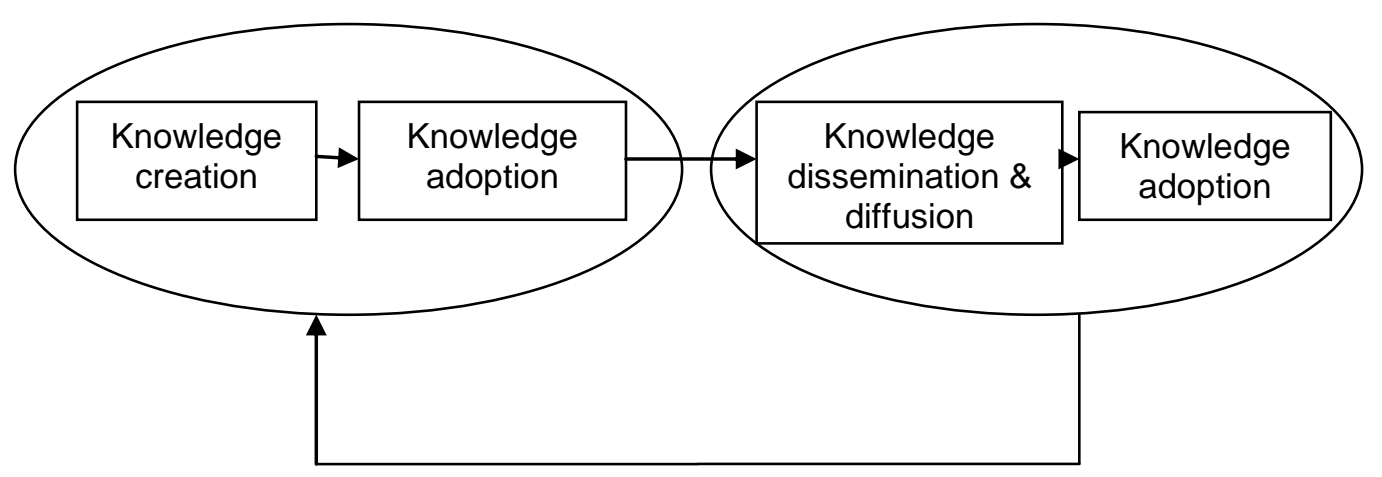

Figure 2: Mode 2 - An alternative model of evidence based practice adapted from Nutley and Davies (2000) p325.

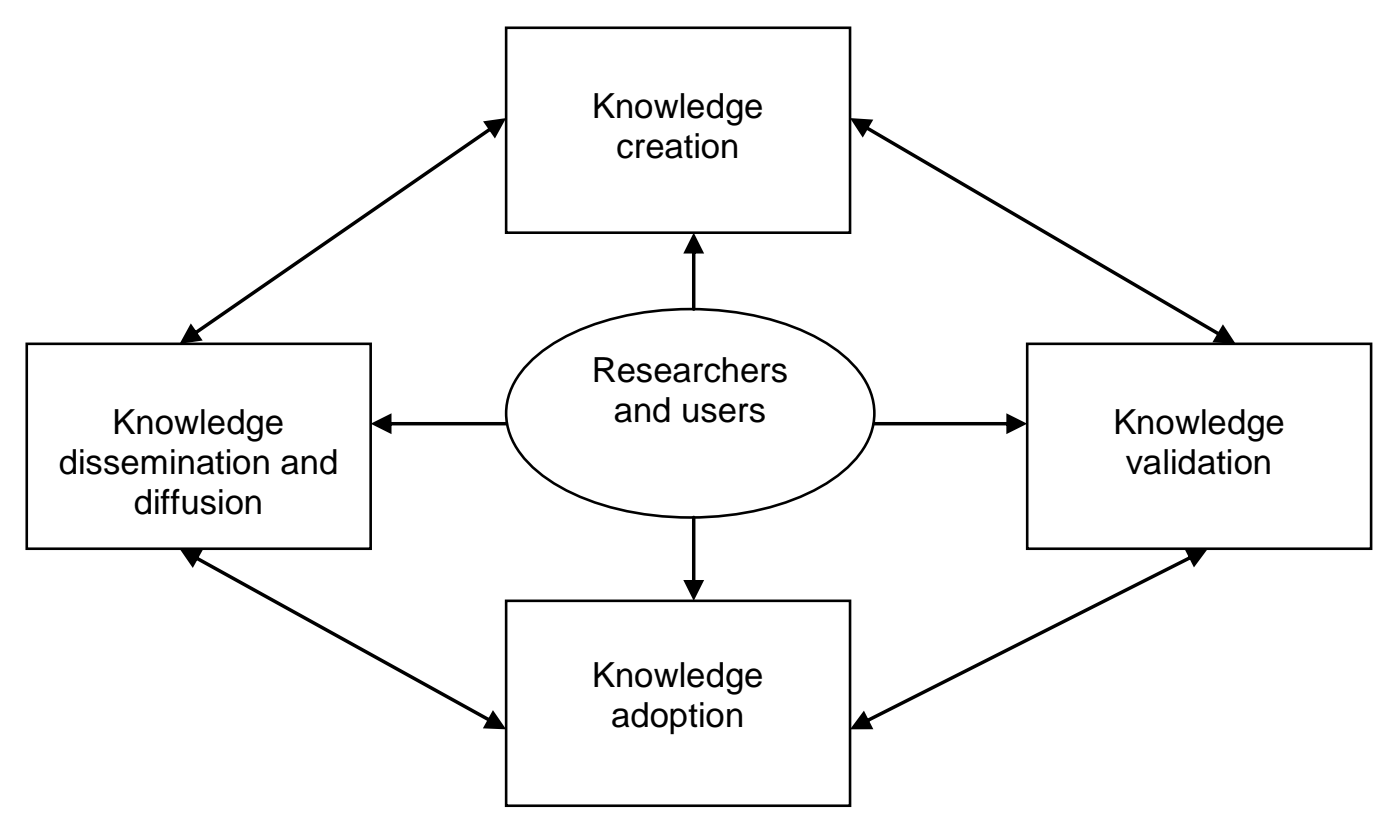


Evidence-based health care is, at its simplest, the idea that the care that health professionals provide should be based as closely as possible on evidence from well-conducted research into the effectiveness of health care interventions, thereby minimizing the problems of underuse, overuse, and misuse (Walsh \& Rundall, 2001).

\subsection{Conclusion}

The evidence is clear that the effectiveness of specialist early intervention teams is valuable for people that have psychosis. The benefits of reducing the duration of untreated psychosis are well documented in the literature. Any attempt to implement an evidence based practice in a clinical setting should consider the individual attitudes and organisational culture and climate. Most of the research conducted on mental health and clinician attitudes has been done in the United States, However it is difficult to draw conclusions from how services work in the US as in England and Wales as there are different structures for the delivery of health and social care and clinical training is more standardised which is largely due to professional bodies. There is currently a lack of research on the implementation of evidence based practices within early intervention teams in the England and Wales. The research that does exists suggests that attitudes of senior staff may be an important element of implementation but that wider contextual conditions relevant to the practitioners organisation may also be important features. This understanding derived from reviewing and analysing the available literature then led me further to consider the use of quantitative and qualitative methodologies and to attempt the integration of these data to allow for an increased understanding of implementation of evidence in early intervention services. In the chapter that follows I lay out in more detail my rationale for considering these methodologies and show how I used the related methods to conduct a study of early intervention in psychosis practitioners in England and Wales. 


\section{CHAPTER 3: Methods}

\subsection{Introduction}

In this chapter I describe the aims and the research design for my study of evidence implementation in early intervention mental health services in England and Wales. I provide a critical review of the methodological approaches that were considered and explain why a mixed methods approach was selected as the preferred approach to address these particular research questions. I will briefly discuss the debate on the divergent views on qualitative and quantitative methods and the emergence of mixed methods as a new paradigm. I will provide examples of how mixed methods designs have been used successfully. I describe in detail the design of the current study and my application of mixed methods to generate data for the purposes of conducting an analysis of evidence implementation in early intervention mental health services. In this chapter I present my rationale for choosing to use an explanatory sequential mixed method design to generate quantitative and qualitative data for analysis in this thesis. The limitations of this research design will be considered later in chapter 6 (discussion).

\subsection{Aims and Research Questions}

The overall aim of this research was to develop an in-depth understanding of implementation of evidence based practices in early intervention mental health services in England and Wales. This was achieved by examining attitudes and implementation of lead practitioners to evidence based practice in early intervention mental health services in England and Wales and in-depth interviews with a range of lead practitioners to examine everyday experiences of implementation issues, for example sustainability and fidelity. 
The research questions are:

1. What are the attitudes of early intervention lead practitioners in relation to evidence based practice?

2. To what extent is service context and culture relevant to practitioner's experiences of adopting evidence based practice?

3. What do early intervention lead practitioners say are the crucial factors in sustaining or not sustaining practices in their teams in England and Wales?

4. What do early intervention lead practitioners say are the facilitators and barriers to implementation of evidence based practices in their services?

\subsection{Pragmatism as a Research Paradigm}

A paradigm could be regarded as an "accepted model or pattern" (Kuhn, 1962, p. 23), as an organising structure, a deeper philosophical position relating to the nature of social phenomena and social structures.

Pragmatism, when regarded as an alternative paradigm, sidesteps the contentious issues of truth and reality, and accepts philosophically, that there are singular and multiple realities that are open to empirical inquiry and orients itself toward solving practical problems in the "real world" (Creswell \& Plano Clark, 2007, pp. 20-28 \& Dewey, 1925). In that sense, pragmatism allows the researcher to be free of mental and practical constraints imposed by the "forced choice dichotomy between post-positivism and constructivism" (Creswell \& Plano Clark, 2007, p.27), and researchers do not have to "be the prisoner of a particular [research] method or technique" (Robinson, 1993, p. 291).

\subsection{Epistemological Stance}

Epistemology is concerned with the relationship between the knower and the known (the researcher and the participant). Positivists and post-positivists perceive this relationship as being objective with dualism or separateness existing between the knower and the known. On the other hand, constructivists perceive research knowledge as subjective, with researchers and participants 
working together to co-construct social realities. From a pragmatic angle, pragmatists challenge this distinct contrast between objectivity and subjectivity. They believe that epistemological issues exist on a continuum rather than two opposing poles (Teddlie \& Tashakkori, 2009).

The research reported in this thesis adopted a contextualist approach, which is a perspective that lies between essentialism and constructionism (Braun \& Clarke, 2006). Contextualism is another epistemology which has a foot in both camps (Henwood \& Pidgeon, 1994). Tebes cites Pepper (1942) to describe the central metaphor of contextualism as 'the human act in context' (2005:216). It can be seen as a version of constructionism in that it doesn't assume a single reality, and sees knowledge as emerging from contexts and reflecting the researchers positions, so that's its local, situated and therefore always provisional (Madill et al., 2000; Tebes, 2005). However, this approach does retain an interest in understanding truth, and hence has a realist dimension. It argues that while no single method can get to the truth (Tebes, 2005), knowledge will be true in certain contexts. So contextualism retains a notion of 'the truth' which constructionism rejects.

\subsubsection{Axiological Considerations: Value Free vs Value Bound}

Positivists believe that inquiry is value free, whereas constructivists believe that inquiry is value bound (Teddlie \& Tashakkori, 2009). Post-positivists acknowledge both the value-ladenness and the theory ladenness of the facts (Raichardt \& Rallis, 1994). Despite this recognition (and to a large degree because of it), post-positivists have devoted considerable effort to developing methods whereby internal and external validity of their conclusions can be enhanced (e.g. Cook \& Campbell, 1979; Shadish et al., 2002). Pragmatists believe that values play a large role in conducting research and in drawing conclusions from their studies but they see no reason to be particularly concerned about it. Cherryholmes (1992) stated:

"For pragmatists, values and visions of human action and interaction precede a search for descriptions, theories, explanations and 
narratives. Pragmatic research is driven by anticipated consequences...Beginning with what he or she desires, our pragmatist would pick and choose how and what to research and what to do". (pp.13-14).

Pragmatists decide what they want to study based on what is important within their personal value systems. They then study that topic in a way that is congruent with their value system, including units of analysis and variables that they feel are most likely to yield interesting responses (e.g. Tashakkori \& Teddlie, 2009). This description of pragmatists' behaviours is consistent with the way that many researchers actually conduct their studies.

Howe (1988) suggests that researcher should forge ahead with what works. Truth, he states, is a normative concept, like good. Truth is what works. This appears to be the prevalent attitude in mixed methods research. Howe's argument seems to suggest that only pragmatists, or those not wedded to either paradigm, would attempt to combine research methods across paradigms. But this does not address the issue of differing ontological assumptions of the two paradigms.

\subsubsection{Ontology: Essentialist or Realist}

In this thesis I have adopted a contextualist approach, a perspective that sits between essentialism and constructionism (Braun \& Clarke, 2006).

Braun and Clarke (2013) explain that thematic analysis can be an essentialist approach or a constructionist approach. An essentialist approach would suggest that data reflects experiences, meanings and the reality of participants. In contrast, a constructionist approach would examine the ways in which events, realities, meanings and experiences are the effects of a range of discourses operating within society.

They also explain that thematic analysis can be a 'contextualist' method, sitting between the two poles of essentialism and constructionism, and characterised by theories, such as critical realism (e.g. Willig, 1999), which recognize the ways individuals make meaning of their experience and in turn the ways the 
broader social context impinges on those meanings, while retaining focus on the material and other limits of 'reality'. Therefore, thematic analysis can be a method that works both to reflect reality and to unpick or unravel the surface of 'reality'. This thematic analysis was conducted in a realist style, reporting participants own reality and experiences as they were told. Realism assumes a knowable world, which is comprehensible through research that the truth (and there is only one) is 'out there' and can be accessed by the appropriate application of research techniques. Hence realism for me means that we can ascertain the truth but only if we use the right tools. Braun and Clarke use the analogy of looking at realism.

"...realism would be looking at a view through a perfect glass window in your house. The information access from the perfect glass window corresponds exactly to what really is outside - if you go outside, the path and garden you have seen would be there; and thus verify the truth you have viewed. Your window has given you a way to determine the reality that exists beyond it..." (Braun \& Clarke, 2013, p. 28).

\subsubsection{Inductive or Deductive}

A second key division that has centred on research is whether analysis of data should be inductive or deductive. Deductive theory represents the commonest view of the nature of the relationship between theory and social research (Bryman, 2012). Figure 3 below from Bryman (2012) captures the essence of the difference between inductivism and deductivism.

Quantitative researchers commonly use methods such as surveys and questionnaires to generate data that can be assigned numerical values and subjected to statistical analysis. May (2001) suggests that this approach is often criticised for putting words into participants' mouths. Such methods fundamentally test preconceptions of the researcher, and one is unlikely to discover new findings unless actively looking for them. In contrast, with the popularity of approaches such as grounded-theory, which focuses upon 'discovery' of theory which 'emerges' from data (Glaser \& Strauss, 1965), qualitative research has usually been seen as inductive, allowing insights 
beyond the preconceived ideas of the researcher, though more deductive qualitative approaches such as framework analysis (Pope, Ziebland \& Mays, 2000) are common in health research. I acknowledge too that the framework also allows for inductive analysis.

In pragmatism, the approach may combine deductive and inductive thinking as the researcher collects, analyses and mixes both quantitative and qualitative data. In this thesis I have been be guided by a combination of prior theory and theory as produced from the themes I have generated via my analysis of data.

\subsection{Reflective Commentary}

Reflexivity carries the connotation that researchers should be self-aware about the implications of their research methods, values, biases, and decisions for the knowledge of the social world they generate (Bryman, 2012).

Berger (2015) denotes that researchers should increase focus on selfknowledge to better understand the role of the self in the creation of knowledge.

Reflexivity plays an important role in qualitative studies particularly in the conduct of interviews. I was very conscious from the very outset of the study to build up a good rapport with my participants. My previous research experience working as a research assistant looking at the culture and climate of CMHTs where I interviewed team managers in NHS trusts helped me to understand how teams were structured and how busy managers can be. I used my previous knowledge and my awareness of how research and researchers may not be the usual day to day priority for practitioners. Practically this means being persistent in pursuing data and flexible in relation to availability to speak with busy workers.

In my role working on the Mental Health Research Network Cymru, I felt I was well connected with academics across the board in mental health research. Networking at conferences was very advantageous and provided opportunities for collaboration with researchers and colleagues alike. I felt I was able to exploit my existing social ties particularly with Early Intervention in Psychosis 
(EIP) leads in England and Wales for the purposes of identifying a sample and collecting data for the current study.

My own reflections particularly my research experience enabled me to recognise how my own biases may impact on the research process. My own philosophical position holding positivist beliefs about research was challenged as I developed my research questions and begin to appreciate the possibilities of other approaches. Pragmatism allowed me to be free of constraints from positivism and constructivism domains. Embarking on analysis I was conscious that I needed to give equivalence to both sets of data. This reflection enabled me to increase my self-awareness in the data integration phase so that I was able to explore deeper meanings of what practitioners were saying beyond the impact of attitudes as barriers to utilising knowledge and skills.

Figure 3: Deductive and inductive approaches to the relationship between theory and research.

Deductive approach

Inductive appr

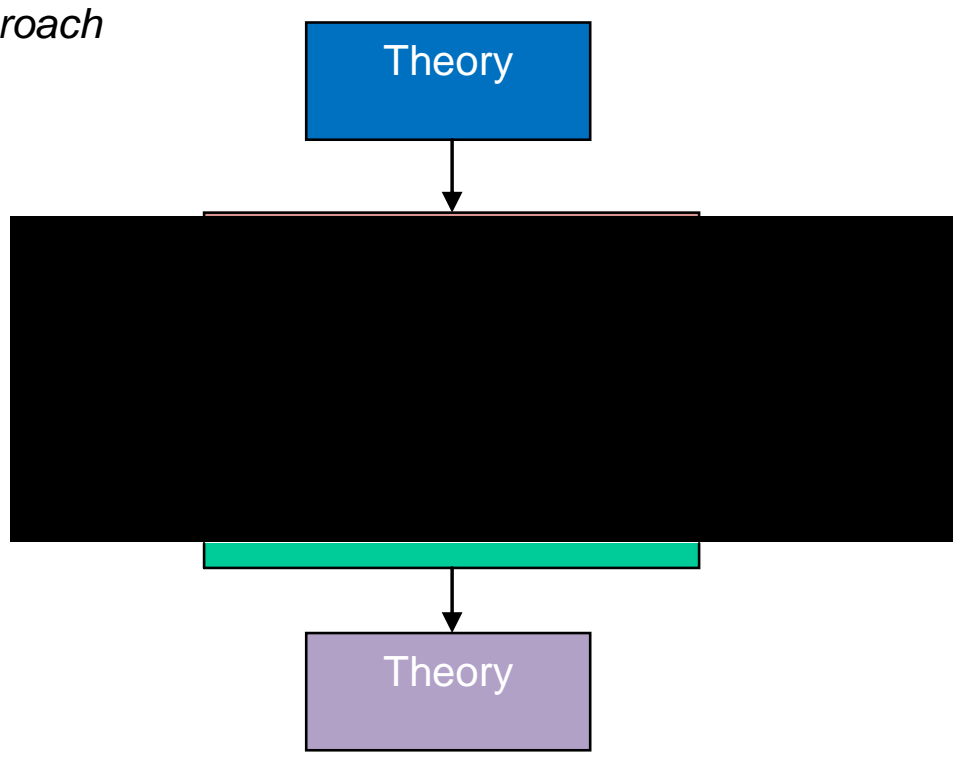

Adapted from Bryman (2012), p.26 


\subsection{Three Communities: Continua, not Dichotomies}

The goal of mixed methods research is to not replace either of these philosophical approaches, but to rather draw from the strengths and minimise the weaknesses of both single research studies and across studies. Johnson and Onwuegbuzie (2004) envisage them on a continuum with qualitative research anchored at one pole and quantitative researcher anchored at the other with mixed methods research covering the large set of points between. If one prefers to think in categories then mixed methods sits in a new third chair with qualitative research anchored on the left side and quantitative research sitting on the right side. It has been argued that mixed methods as the third paradigm can also help bridge the division between qualitative and quantitative research (Onwuegbuzie \& Leech 2004a).

\subsection{Adopting a Mixed Methods Approach}

Regardless of paradigmatic orientation, all research in the social sciences represents an attempt to provide warranted assertions about human beings (or specific groups of human beings) and the environments in which they live and evolve (Biesta \& Burbules, 2003). In the social and behavioural sciences, the aim is to understand the various aspects including: experiences, intentions, attitudes, culture and holistics.

Today's research world is becoming increasingly interdisciplinary, complex, and dynamic; therefore, many researchers need to complement one method with another. Researchers need a solid understanding of multiple methods used by other scholars; this will facilitate good communication and collaboration in order to provide superior research (Johnson \& Onwuegbuzie, 2004). By taking a nonpurist or compatible mixed position it allows researchers to mix and match design components that offer the best opportunity to answer specific research questions.

Teddlie and Tashakkori (2009) suggest that researchers in the social and behavioural sciences can be categorised into three groups. The first group are quantitative orientated social and behavioural scientists primarily working within 
a positivist paradigm and principally interested in numerical data and analysis. Second, the qualitative orientated social and behavioural scientists primarily working within a constructivist paradigm and principally interested in narrative data and analysis. Third, the mixed methodologists working primarily within the pragmatist paradigm with an interest in both narrative and numeric data and their analysis.

Combining both methods of quantitative and qualitative approaches is mixed methods research, and has been defined by Tashakkori and Cresswell (2007) as:

"Research in which the investigator collects and analyzes data, integrates the findings, and draws inferences using both qualitative and quantitative approaches and methods in a single study of a program of inquiry" (p. 4).

Mixed methods research has been called the third path (Gorard \& Taylor, 2004), the third research paradigm (Johnson \& Onwuegbuzie, 2004) and the third methodological movement (Teddlie \& Tashakkori, 2003) by various individuals in the field. Hence the overall central premise of a mixed method is that the use of a quantitative and qualitative approach combined. It can provide a better understanding of research problems and complex phenomena rather than either approach set alone (Creswell \& Clark, 2007).

The most common and well-known approach to mixing methods is the triangulation design (Creswell, Plano Clark et al., 2003). The purpose of the design is "to obtain different but complementary data on the same topic" (Morse, 1991, p. 22) to best understand the research problem. Although the design is the most popular mixed methods design, it's probably the most challenging. Triangulation in itself has no guarantee of internal and external validity. It's real value is not that it guarantees conclusions about which we can be confident but that it prompts researchers with a more critical stance towards their data (Fielding \& Fielding, 1986). Tashakkori and Teddlie (2003) denote 
that one limitation of mixed methods is a perceived incompatibility of mixing methods that have two opposing world views.

The explanatory design also known as the explanatory sequential design is illustrated below in Figure 4 and is the approach chosen for the current study. My study combined a quantitative survey with qualitative semi-structured interviewing which are two distinct methods categorised by two different paradigms to explore different aspects of the same or different phenomena. The design is sequential (e.g. first the survey then the interviews) with the methods being independent of each other but with each method having equal status. Conducting the research in a sequential way allowed me to complete an initial analysis of the EBPAS and then use this information to select participants for the semi-structured interview stage. The qualitative data allowed me to gain insights into the richer context and generate understanding of issues around implementation (sustainability and fidelity).

Figure 4: Explanatory Design: Participant Selection Model (Qualitative emphasized) taken from Creswell, 2007, p.73.

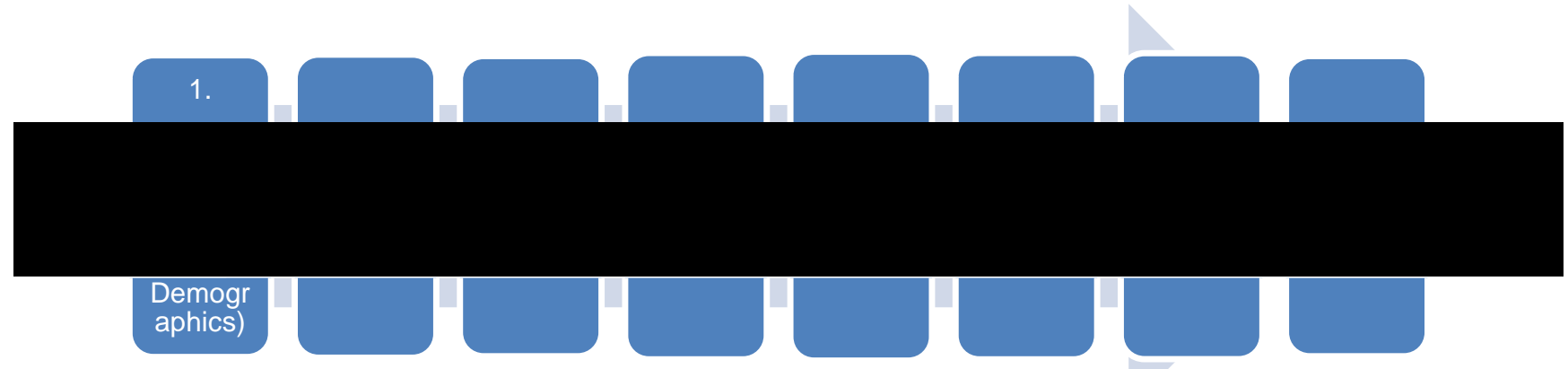

\subsection{Integration of the Survey and Interviews}

A key benefit of mixed methods research is that both quantitative and qualitative strengths can be incorporated into a study to minimise the effects of the weaknesses of both approaches (Johnson \& Onwuegbuzie, 2004).

Teddlie and Tashakori (2009) suggest that integration does not necessarily mean creating a single understanding on the basis of results. 
Integration in mixed methods research denotes making meaningful conclusions on the basis of consistent or inconsistent results. The term incorporates linking, elaboration, completeness, contrast, comparison and alike (Teddlie \& Tashakori, 2009).

The explanatory sequential mixed methods design used in this thesis is where one phase is followed by another. The first phase is quantitative followed by qualitative. The second phase is connected to the first phase. Connection of data is between the two phases. This design was the best design for this study because I wanted to follow-up with the same group of practitioners and to use what I gleaned from the initial analysis to focus the qualitative research. The process was a sequential explanatory mixed methods design (Creswell \& Plano Clark, 2007) see figure 4 above.

The exploratory design uses a qualitative method first and a quantitative method second. It is often used in instrument development or taxonomy development. Instrument development was not a focus of this particular study.

Data integration occurred at the interpretation phase where there was a comparison of data. I outline the process I engaged in to achieve integration of analysis in the section below. I found that this approach enabled me to gain a deeper understanding of practitioners attitudes towards evidence based practices. The qualitative data was used to give a richer context to the quantitative data analysis. By interviewing practitioners and having their input enabled me to enhance the validity of findings. This enabled me to gain more meaning from the data, than the single mono-method.

Data integration and interpretation did identify some conflicting results between both samples, but also identified areas of agreement and using qualitative data gave an additional context to quantitative data that was not apparent within this as a single method.

The mixed methods sequential explanatory design consists of two distinct phases: quantitative data collection followed by qualitative data collection 
(Creswell, Plano Clark, et al., 2003). For the integration of quantitative and qualitative components, I decided to follow O'Cathain et al.'s (2010) paper in which the authors see a role in examining a subset of the data. Hence the subset is for integration purposes only. A unique aspect of mixed methods studies is the availability of both qualitative and quantitative data on the same cases (O'Cathain et al., 2010). The mixed methods matrix was used to look at the main summary findings from the quantitative component of the survey and the main themes from the qualitative aspect of the study to examine whether the extent to which the findings from each strand agreed with each other. The subset of the data was the statistical significant findings of the EBPAS scales requirements, openness and total EBPAS score along with the individual participants. I compared participants' quantitative responses with their interview transcript. The subset was relevant to addressing research question 2, 'to what extent is service context and culture relevant to practitioner's experiences of adopting evidence based practice?'

The integration offered a deeper description and explanation to be offered that the survey did not but these may also have shown where the limits are to assessing attitudes when other factors are in play e.g. organisational factors.

In the matrix (see Appendix 15 - summary of findings from quantitative and qualitative study) the table comprises of five columns (participant ID, findings from quantitative study, and findings from qualitative study, aspects upon which quantitative and qualitative studies agree or disagree). Within the mixed methods matrix, the rows represent the cases for which there is both qualitative and quantitative data and the columns display different data collected on each case (O'Cathain et al., 2010).

I used the mixed methods matrix to look at statistically significant quantitative findings and qualitative themes (see Chapter 6). Two key significant variables in the quantitative component of the survey were EBPAS requirements and openness sub scales. 
Interviews were conducted with 18 participants. The matrix was used to see and show where findings from the quantitative and qualitative components diverged, agreed or complement each other.

In particular, it helped to identify negative cases (where data in the analysis doesn't fit with the conclusions the analysis is coming to) within the qualitative analysis to facilitate understanding.

\subsection{Qualitative Integrity}

Guba and Lincoln (1981) stated that while all research must have truth value, "applicability" and "neutrality" in order to be worthwhile, the nature of knowledge within the rationalistic (or quantitative) paradigm is different from the knowledge in naturalistic (qualitative) paradigm. Hence Guba and Lincoln (1981) propose that it is necessary to specify terms and ways of establishing and assessing the quality of qualitative research that provide an alternative to reliability and validity. They propose two primary criteria for assessing a qualitative study: trustworthiness and authenticity.

\subsubsection{Validity and Trustworthiness}

In conducting interviews there has been a long debate in relation to methodological rigor within qualitative research and is contested within the qualitative research community (Morse, 1999 \& Sparkes, 2001). Silverman (2005) says that trustworthiness is made up of for criteria each of which has an equivalent criterion in quantitative research:

1. Credibility, which parallels internal validity;

2. Transferability, which parallels external validity;

3. Dependability, which parallels reliability;

4. Confirmability, which parallels objectivity. 
Validity

'By validity, I mean truth: interpreted as the extent to which an account accurately represents the social phenomena to which it refers'.

(Hammersley, 1990:57).

Reliability

'Reliability refers to the degree of consistency with which instances are assigned to the same category by different observers or by the same observer on different occasions. (Hammersley, 1992:67).

\subsubsection{Credibility}

The credibility of findings involves establishing that the results of qualitative research are credible or believable from the perspective of the participant in the research (Bryman, 2012). The purpose of qualitative research is to describe or understand the phenomena of interest from the participants' eyes. Credibility relates to the truth of my findings thus using direct quotes from participants to illustrate or develop themes can enhance credibility. From the outset of my research I did not aim to conduct member checks. Member checking refers to the practice of checking analysis with the participants (Seale, 1999). My rationale for not including member checks is because this is a contested notion in qualitative research which McLeod (2001) explains can also lead to practical problems. For example, some participants may be reluctant to engage in the process: they may not have time to participate in the 'extra' process or perceive no value in doing so. My view is that member checking also increases participant burden and could lead to recruitment problems from already busy participants who may be reluctant to commit to further effort on their part.

\subsubsection{Transferability}

Transferability refers to the degree to which the results of qualitative research can be generalized or transferred to other contexts or settings (Bryman, 2012). From a qualitative perspective transferability is primarily the responsibility of the one doing the generalizing meaning the researcher. The qualitative researcher can enhance transferability by doing a thorough job of describing the research 
context and the assumptions that were central to the research. The person who wishes to "transfer" the results to a different context is then responsible for making the judgment of how sensible the transfer is.

\subsection{Ethics}

Ethical standards prescribe that respondents should never be coerced to take part in a study and that participation should be free, voluntary and fully informed (Sarantakos, 2005). A vital element of the ethical considerations of the study was to ensure that research participants were sufficiently informed about the study and were able to give informed consent to participate. Consent for participation in the online survey was assumed based on the participant choosing to return the survey to the researcher.

The consent agreement for the semi-structured interview was sent to the participant with the information sheet on the study prior to the telephone interview commencing. The participant information sheet explained in plain English what the study was about, what would be involved if they agreed to take part, and what would happen to the information that was collected. The information sheet explained that it was intended to include some anonymised quotes from the interviews in the thesis and in publications.

The consent agreement was emailed back to the researcher or posted to the researchers Swansea University work address. Consent to audio record the interview sessions were rechecked prior to the telephone interview. Participants were reminded that they were free to withdraw from the study at any time which was their right to do so without giving a reason, and it also provided contact details for the academic supervisor. The interviews were voluntary and were arranged at a time that was convenient for the participant and researcher.

\subsubsection{Ethical Approval}

A protocol was submitted to Swansea University College of Human and Health Sciences research ethics committee (REC) in October 2014, and approval was 
granted on 30 October 2014 (see Appendix 5). Prior to the protocol being submitted a full application was submitted to the National Research Ethics Service ethical review NRES Committee North West Lancaster in August 2014 (see Appendix 6). From the application it was evident that the research was being conducted on NHS staff only and not on patients. Therefore the advice from the REC manager at Hywel Dda University Health board was that approval should be sought via Swansea University Research Ethics Committee and hence this is the advice that I acted upon.

\subsubsection{Anonymity and Confidentiality}

The basics of ethical standards in social research are privacy, anonymity and confidentiality (Bryman, 2012). Bryman (2012) suggests that researchers should not be expected to ask personal and sensitive questions if they realise that respondents do not feel comfortable about revealing such information. When anonymity is upheld the researcher ensures that the name of the participant does not appear on the research instrument or the data. Questionnaires or interview guides have no names. Confidentiality is maintained by ensuring that information made public will not include the name of the participant, or make it possible that the information can be linked with a participant.

One ethical concern I had was related to the protection of individual participant's anonymity. I anonymised the participants in the study by giving them a pseudonym; however there is always a risk that readers with insider knowledge might be able to identify locations or individuals. To reduce this risk I provided psedudonym e.g. "Martin, nurse from England".

All participants were assigned a study number and only the researcher had access to this key. The participants were identified by the research study number on all transcripts and data extracts. All identifiable material was removed from all interview transcripts. Pseudonyms were given to the participants who were selected for the semi-structured interviews. Maintaining confidentiality was an important issue, and care was taken to ensure that all the 
data collected from research participants were password protected. The PhD project followed the relevant guidance which is fully in accordance with Swansea University's Research Governance Guidelines and the Data Protection Policy (based on the 1998 Data Protection Act), and the related guidelines Research Projects and Personal Data: Guidance on use of personal data in staff and student research' and the Security of Personal Data: Guidance to staff and students of their responsibilities for ensuring the security of personal data. The six Caldicott Principles were upheld throughout the project and writing up of the thesis (DoH, 2010).

\subsection{Sample Population}

My aim was to obtain a total population sample for the survey and a purposive sample for the research interviews. The total population sample included all health care organisations comprising of staff that work in an early intervention multi-disciplinary teams in mental health which comprise of hybrid managers (managers that are in a clinical role). These could be community psychiatric nurses (CPNs), psychiatrists, social workers, occupational therapists, psychologists. Participants were excluded if; they did not work in an early intervention team, or were working in a role with no leadership responsibility for the team. I made contact with the national lead for England via the initiative to reduce the impact of schizophrenia (IRIS) for the purposes of establishing a sampling frame for the study (see section 3.12) who predicted that a sample of 80 teams would be achievable based on the response of the last IRIS audit. I made contact with the lead for Wales via the 1000 Lives Wales network for the purposes of establishing a sampling frame for the study (see section 3.12) who predicted that participation of 16 clinical leads would be achievable.

At the design stage of the study and following discussions with colleagues working in the relevant fields it was recognised that there was significant risk that early intervention practitioners working in primary and community care settings might be unwilling to participate in the interviews. This was anticipated due to the time pressures and workload faced by those staff, and was 
exacerbated by other demands on their time. Hence, to alleviate time pressures the survey was designed to be relatively short and convenient to complete.

Phase one of the study was the quantitative survey Evidence Based Practice Attitudinal Scale (Aarons, 2004) demographics and phase two of the study was the qualitative semi-structured interviews with early intervention practitioners. The findings from the survey phase one were used on the basis for the selection of a purposive sample for the semi-structured interviews in phase two. The 18 interviewees were selected using a rudimentary sampling frame based upon the following criteria; EBPAS scores (high and low), urban and rural sites, newly established early intervention teams (less than one year) and more established early intervention teams (more than one year). This sampling strategy for the semi-structured interviews allowed interviewees to be selected purposively which is a non-probability form of sampling i.e. the researcher does not seek to sample research participants on a random basis (Bryman, 2012) in terms of the criteria (see above) that ensured that practitioners with different experiences of early intervention which I wanted to learn more about.

\subsection{Negotiating Access to Early Intervention Practitioners}

I established contacts with administrative and clinical leads from the initiative to reduce the impact of schizophrenia (IRIS) and 1000 Lives Wales network. The IRIS was the inspiration behind the ground breaking reforms across England which has seen early intervention for psychosis become a standard feature of mental health care. The 1000 Lives Wales network is the national improvement body for NHS Wales delivered by Public Health Wales. The aim is to support the NHS to improve outcomes for people using services in Wales.

I attended the 1000 Lives Improvement conference in North Wales where I had the opportunity to meet key individuals in England such as the Joint National Early Intervention Programme Lead for the National Mental Health Development Unit (NMHDU) and the early intervention clinical development 
lead for Worcestershire early intervention service. For Wales I made contact with the clinical lead for psychosis, recovery who was a member of the 1000 Lives Plus network. Early introductions and communications with key contacts above helped me tremendously in moving forward with 'in-principle' access.

In-principle access was negotiated with the early intervention national leads from the psychosis initiative to reduce the impact of schizophrenia (IRIS) in England, and the 1000 Lives Plus programme in Wales who had distribution lists of contact details of early intervention services around the country. The research PhD project was formally supported at the IRIS national leads' meeting and it was agreed to support gaining access to as many teams via the national leads as possible via their networks. The national lead for the IRIS Early Intervention in Psychosis (EIP) network suggested that contact with individual national leads should be made via them rather than directly. This was to avoid teams feeling overwhelmed by requests and it was thought that a better response rate would be obtained if requests came directly from the national group. The group had a substantive amount of requests from other researchers and audits were underway at that time.

The same arrangement was followed in Wales with the 1000 Lives Plus network clinical lead for psychosis and recovery. The plan was that an email would be sent to teams which included information about the study and a link to the online survey questionnaire with a two week deadline for completion. Reminders were sent on behalf of the national leads in order for a greater response rate.

\subsection{Surveys}

Surveys can be designed to measure certain phenomena (events, behaviour, attitudes) in a population of interest, by using reliable and valid measure is to look at the key phenomena of interest. Surveys are methods of data collection in which information is gathered through oral or written questioning (Sarantakos, 
2005). Oral questioning is known as interviewing and written questioning is accomplished through questionnaires (Sarantakos, 2005).

\subsubsection{Design, Conduct, Advantages and Disadvantages of Surveys}

Questionnaires can either be handed out to the respondents personally, or are sent to them by mail which includes three main parts which are the cover letter, the instructions and the main body of questions to be addressed or rated. In the current study a link to the online questionnaire was sent to potential participants via email.

Sarantakos (2005) say that the cover letter can influence the response rate in the way the questionnaire is presented and introduced and the assurances given to respondents determine to a large extent the probability of their retuning the questionnaire and answering all of the questions.

The instruction about how to compete the questionnaire is very important as inadequate instructions are one of the major sources of non-responses which should be avoided (Sarantakos, 2005). The main body of the text of the questionnaire should include the questions to be answered paying attention to content, structure, wording, flow and format. Question development is essential for data gathering in self-administration questionnaire.

Sarantakos (2005) says that questionnaires, as methods of data collection, have strengths which the researcher must be aware of. The advantages of questionnaires, as methods of data collection are as follows: they produce quick results, they can be completed at the respondents' convenience and they offer great assurance of anonymity.

Paper surveys tend to be costly, even when using a relatively small sample and the costs of a traditional large-scale survey using mailed questionnaires can be significant (Bachmann \& Elfrink (1996). The use of online surveys circumvents 
this problem by eliminating the need for paper and other costs, such as those incurred through postage and printing (Llieva et al., 2002).

Survey questionnaires present some weaknesses and these are: they do not allow for probing, prompting or clarification of questions, they do not provide opportunities for motivating the respondent to participate in the survey or to answer the question, it is not possible to check whether the question order where required was followed and due to lack of supervision, partial response is quite possible (Sarantakos, 2005).

I selected Survey Monkey as an online survey questionnaire tool because I thought this would be more convenient for busy practitioners, it was available via a university wide subscription and given that my $\mathrm{PhD}$ was not funded resources were limited in terms of time and money. Online surveys can also save money by moving to electronic medium from paper and saving costs on postage and possible delays or loss of returns (Bachmann \& Elfrink, 1996). An added incentive was that the College of Human and Health Sciences in Swansea University had gold membership access to Survey Monkey for researchers in the college meaning there would be no extra cost to me as a researcher. 


\subsection{Evidence Base Practice Attitudinal Scale (EBPAS, Aarons, 2004)}

Any effort to implement EBP in clinical settings and services needs to consider individual attitudes and organisational culture and climate. Organisational social context includes the norms and expectations (i.e. culture) of the organisation for its members as well as the psychological impact of the work environment on the individual workers (i.e. climate) (Aarons, Glisson \& Hoagwood, et al., 2012). Factors such as level of education and level of professional experience have been found to be associated with attitudes towards adoption of EBP (Aarons, 2004). The contextual questions such as type of service, policies may be important in understanding adherence or lack of to practice change (Glisson, 2002; Strupp \& Anderson, 1997).

In her thesis Cunningham (2013) looked at dissemination methods and attitudes to family intervention for psychosis in trainee clinical psychologists using the EBPAS and her study findings showed that the openness and requirements subscales were significantly positive predictors of attitudes to family intervention. The finding that trainees' responses to the requirement subscale, which measures the impact that local (both supervisor and organisational level) and national requirements had on the likelihood of them adopting family intervention, was a significant factor which had implications for dissemination and implementation of family intervention.

In the current study the EBPAS scale (Aarons, 2004) was chosen to help assess the implementation of evidence based practices in early intervention services (Appendix 2). The EBPAS scale is a well validated, 15-item self-report questionnaire that assesses constructs related to the implementation of Evidence Based Practices (EBP). The four dimensions of attitudes adoption of EBP had been identified previously by Aarons (2004): (1) intuitive appeal of EBP (2) likelihood of adopting EBP given the requirements to do so, (3) openness to new practices, and (4) perceived divergence of usual practice with research-based/academically developed interventions. Participants were asked 
to respond to each of the 15 questions which enquired about attitudes to using new types of therapy, interventions, or treatments by noting the extent to which they agreed with each item using a five-point Likert scale that ranges from 0 (not at all) to 4 (to a very great extent). The scale was initially designed for use in child and family mental health settings within the United States. I have replicated the use of Aarons EBPAS in an England and Wales context with early intervention practitioners to assess their attitudes to adopting an EBP. Aarons' (2004) confirmatory factor analysis on the EBPAS found that reliability was good (Cronbach's alpha $=0.77$ ), with subscale alphas ranging from 0.59 to 0.90 and the measures validity is supported by studies of EBPAS score associations with mental health clinic structure and policies (Aarons, 2004), culture and climate (Aarons \& Sawitzky 2006) and leadership (Aarons, 2006).

Aaron's included professional status in his study which indicated whether the respondent was an intern or employed professional. Interns are those still completing their education and transitioning into professional roles (Aarons, 2004). I decided not to include this in my survey as I thought it would be very unlikely in England and Wales to find individuals working in the capacity of an intern.

The terminology in Aarons' EBPAS attitude scale was altered to reflect the England and Wales context, so that the practitioners would relate to the services in the England and Wales. Aarons conducted his research with 322 public sector clinical service workers from 51 programs providing mental health services to children and adolescents and their families in the United States of America. Mental health services in the United States are delivered by a range of providers and in range of both public and private settings. Terminology was changed to reflect England and Wales service organisation and delivery. In England and Wales context 'agency' was changed to 'organisation' namely NHS trust and 'state' was changed to 'country'.

Primary disciplines were identified in England and Wales context as psychiatry, nursing, social work, occupational therapy, psychology and other category. The 
"other" category included disciplines that were not one of those mentioned above (e.g. counselling and psychotherapy). The primary discipline in Aarons (2004) study was identified as 'marriage', 'family therapy', 'social work', 'psychology', 'psychiatry' and 'other'. The 'other' category in Aarons study included disciplines that were not one of those mentioned i.e. (criminal justice, drug rehabilitation, education and public health).

There are three main models for delivery of EIP services in England and Wales: the 'standalone', 'hub and spoke' and 'enhanced community mental health team' (see literature review Chapter 2 for definitions). The survey included a service model question listing all three categories along with an 'other category please specify'. The responses were categorised for the most part as 'standalone', 'hub and spoke' or 'enhanced'.

The question in relation to the types of services provided by the respondents organisation which was a multiple response item was operationalised in terms of dummy variables of 'inpatient', 'outpatient', 'day treatment' and 'case management'. All were labelled as $(0=$ no; $1=y e s)$. Due to the multiple responses all items could be included in regression modelling in contrast to single response questions such as service model.

The question, 'Does your organisation adhere to the following practice policies was another multiple response item all the policies were listed and were labelled as $(0=$ no; $1=$ yes $)$.

Upon completion of the EBPAS and demographic information which included country, age, gender, educational level, primary discipline and organisational questions (service model, types of services provided by service model, practice policies, primary service area, years of experience working in mental health services, establishment of early intervention team participants were asked at the end of the survey, 'if you are selected, would you take part in a semistructured interview'? Two tick box options were available, 'yes I would be willing to take part' or 'no I wouldn't be willing to take part'. Participants that selected 'yes' to take part in the interview were then asked, 'if you would like to 
take part in a semi-structured interview at what email address would you like to be contacted on'?

A copy of the questionnaire, including the demographics sheet and EBPAS Kish grid (Kish, 1949) is included in Appendix 2.

\subsection{Piloting the EBPAS and Demographics Survey}

The purpose of piloting a questionnaire (Bryman, 2012) is to help recognize whether questions are clearly worded, given that when an online questionnaire is used the researcher will not be there to explain any uncertainty. It can also help to check if the ordering of questions is appropriate. In this research study the aim of the piloting was to make sure that the terminology and definitions used were appropriate for the target audience and to ensure that the questionnaire could be completed within a few minutes and design, content of the questionnaire and covering note, all identified as important principals of good questionnaire design. The survey questionnaire was piloted and tested with five colleagues who were researchers, academics and practitioners from the mental health research team based at Swansea University.

Feedback from the academics and practitioners involved in the pilot test said that the questionnaire was very straightforward and was completed in a timely fashion. Some respondents suggested minor changes to the wording of some of the demographic questions and these comments were taken on board and adopted. For example, for the gender question two other categories were added 'Prefer not to say' and 'Other (please specify)'. Definitions were provided for three questions in the demographic part of the survey. These were for type of 'model' used in the respondents service (standalone specialist team; hub and spoke; enhanced community mental health team); which types of services are provided by the respondents service model (inpatient, outpatient, day treatment, case management) and primary discipline service area for the respondents organisation (urban, rural, and suburban). 


\subsection{Research Interviews in Qualitative Research}

The term 'qualitative interview' is often used to capture different types of interview that are used in qualitative research (Bryman, 2012). The most common interviews are the semi-structured and unstructured ones. However, it's the flexibility of the interview that makes it so attractive. Britten (1995) describes three types of interview: 'structured interviews' which typically used in quantitative research, 'semi-structured interviews', which are conducted on the basis of a loose structure with open-ended questions and 'depth interviews' which may only cover only one or two topics.

Researchers have focused on how the research interview has particularly strong meanings for the research participant particularly for sensitive topics (Brannen, 1988). The research interview can be a site for the construction of one's moral identity (Presser, 2004).

Interviewing does have its advantages and limitations which I will briefly discuss here. Interviews can be adjusted to meet diverse situations, they attract high response rates, easy administration in that they do not require participants to have the ability to read or handle complex documents, control over time, date and place of interview Sarantakos (2005). Interviews can be conducted exactly as planned regarding time and date and according to specific conditions. Limitations of interviews are the following: costly and time consuming than other methods such as questionnaires, interviews are affected by the 'interviewer' factor and the possible bias associated with it, interviewing is less convenient than other methods such as questionnaires and sensitivity issues in relation in that participants prefer to write about sensitive issues rather than talk about them (Sarantakos, 2005). Research interviews also have to be transcribed and analysed and this takes time and resources.

For the research in this thesis, semi-structured interviews were selected for the qualitative component which consisted of open questions for discussion with early intervention practitioners working in NHS settings. I will describe below the 
construction of interview guides that were used, how the interviews were undertaken and how the data was subsequently handled.

A semi-structured interview refers to a context in which the interviewer has a series of questions that are in the general form of an interview guide but is able to vary the sequence of questions. The questions are frequently somewhat more general in their frame of reference from the typically found in a structured interview schedule. The interviewer usually has some latitude to ask further questions in response to what are seen as significant replies (Bryman, 2012).

Semi-structured research interviews were used for this phase and were considered to be more appropriate than unstructured interviews. A semistructured research interview gives allowances for the participant to raise what is of interest to them and they allow the interviewer to pursue interesting lines of inquiry that the participant raises which had not been covered in the original schedule (Bryman, 2012). Semi-structured interviews also probe follow-up questions and exploration of topics unanticipated by the interviewer, facilitate development of subtle understanding of what happens in the case and why (Mabry, 2009). With semi-structured interviews the researcher has a list of questions or fairly specific topics to be covered, often referred to as an interview guide, but the interviewee has a great deal of leeway in how to reply. The questions may not follow on exactly in the way outlined on the schedule. Also, the emphasis must be on how the interviewee frames and understands issues, what the interviewee views as important in explaining and understanding events, patterns, and forms of behaviour (Bryman, 2012).

The semi-structured interview schedule used for this study focused on sustainability of EBPs, barriers, facilitators to implementing EBPs and fidelity which were based on previous studies by Swain (2010) and Bond (2012) which looked at the sustainability of evidence based practices in routine mental health agencies. My choice of questions were determined by the literature reviewed in particularly sustainability. 
The interviews were conducted with early intervention practitioners to explore everyday experiences of implementation issues within their teams in relation to issues such as suitability and fidelity. A copy of the interview schedule is provided in Appendix 4.The interview schedule started with straightforward questions, such as 'what is your job title' and when did you start this position (month/year)' then moving onto three main component sections. Section A sought participant views on critical factors in sustaining early intervention teams in their organisation and factors that have worked against sustaining early intervention teams. A summary question was asked if there was anything else the participant would like to add that would help in understanding the sustainability (or not) of Evidence Based Practice at their organization. A final question was asked for the respondent to report three things, either in or out of their control that they thought worked against sustaining Evidence Based Practice at their organisation. Section $\mathrm{B}$, asked about barriers and facilitators that their organisation faces with regards to implementing Evidence Based Practices in their service and section $C$ asked about fidelity in early intervention teams (fidelity being defined as the degree to which an intervention was implemented as it was prescribed in the original protocol or as it was intended by the program developers). Prompts were provided if participants didn't mention adherence indicators (e.g. stand-alone service model, dedicated consultant psychiatrist input or care provided up to three years).

The research interviews with early intervention practitioners were held between May and August 2015. The interviews varied in length from 12 to 52 minutes, with the average interview lasting 26 minutes.

Following each research interview, the recording was uploaded to the researchers computer which was password protected based at Swansea University premises. The audio recordings were checked by the researcher to ensure that the recordings were audible and then the original recording was secured on a memory stick which was password protected thus providing protection against loss of data. Following submission of thesis the audio recordings will be deleted from the researcher (JW) computer after five years 
once the doctorate is completed and ready for publication in the University library.

At the design stage, the researcher decided for practical purposes and to ensure a timely completion that a mixture of a confidential professional transcription agency and transcription by the researcher would be used to transcribe the audio recordings. There are advantages of when the researcher transcribes the data as the researcher is immersed in ones dataset, as it was necessary to listen to the recording of each interview several times in order to complete the transcription (Braun \& Clarke, 2003). Another advantage of the researcher transcribing is that they have participated in both the verbal and nonverbal exchanges with the participants. The main disadvantage of this approach was the time taken to complete this stage of the project. Britten (1995) estimates that a one hour interview can take six to seven hours to transcribe and my experience was this proved to be a realistic estimate.

\subsection{Telephone Interviewing in Qualitative Research}

I chose to use telephone interviews as the method to conduct the semistructured interviews and I outline here my reasons for this choice. The advantages of using the telephone include decreased cost (Novick, 2008), increased access to geographically disparate participants (Sturges \& Hanrahan, 2004), increased interviewer safety (Carr \& Worth, 2001; Sturges \& Hanrahan), and the ability to take notes unobtrusively. Telephone interviewing allows participants to remain on "their own turf" (McCoyd \& Kerson, 2006, p.399), permit more anonymity and privacy (Sturges \& Hanrahan), decreases social pressure.

There are limitations of telephone interviewing as the researcher cannot engage in direct observation of the participant. Nonverbal interview data can contain cognitive or emotional content, and are thought to contribute to the richness of data and interpretation of participants' verbal responses (Burnard, 1994; Novick, 2008; Fontana \& Frey, 2005). This limits interviewers in their tools for communication. For instance, as no nonverbal communication is possible, the 
researcher has to say explicitly 'thank you' or 'yes', instead of a nod or a smile. Information conveyed in gestures and actions is undeniably lost via telephone. However, these data may not always be essential or helpful, as nonverbal behaviour can easily be misinterpreted (Burnard 1994; Chapple 1999; Sturges \& Hanrahan, 2004).

I felt that telephone interviews helped reduce the nonverbal cues and potential bias as interviewees could not be influenced by facial expressions as there is no visual feedback. In conducting telephone interviews I felt I had to really listen to the respondents' especially at their pauses, and laugh's as this provided context to what they were saying. Telephone interviews enabled me to overcome the problems of geography and limited finances that would have prevented me from conducting interviews with this dispersed group of participants.

\subsection{Data Collection and Transcription}

For the quantitative phase of the study the data was downloaded from the Survey Monkey website with each participant response numbered. I entered the data manually in SPSS, I double checked the entries.

For the qualitative phase of the study, participants indicated their willingness to participate at a mutually convenient time agreed by the researcher and participant. In each case the researcher emailed the participant the information sheet and consent form in advance inviting them to take part in the second phase of the study. Once interviews had been arranged in each case the participant was asked to give their explicit consent to take part in the study by verbally agreeing to the seven points of consent (see Appendix 7) prior to the interview agreed date and time. All eighteen participants agreed to the interview being audio-recorded via the Olympus digital voice recorder WS-832. 


\subsection{The Online Survey Questions: (Evidence Based Practice Attitudinal Scale and Demographics)}

Anderson and West (1998), Birleson (1999), Damanpour (1991) and Glisson (2002) all denote that provider attitudes towards innovation and change are likely to interact with both individual differences (e.g. professional experience, training) and contextual factors such as organisational structure and organisational type.

The first part of the survey questions (1 to 6 ) which were in relation to the provider demographics such as country, age, gender, educational level (professional qualification, degree, masters degree, PhD, MD, Fellowship and other category please specify included). Questions 7 to 12 were organisational /contextual questions such as service model, types of services, adherence to policies, primary service area, years of experience working in mental health services, years of establishment of early intervention team. Questions 13 and 14 were about the number of care coordinators in the team, the discipline of care coordinators in the team. Question 15 asked about familiarity to 'evidence based practices' and 'empirically supported treatments' In assessing attitudes towards adoption of an evidence based practice (EBP) lack of familiarity with the concept of EBP requires consideration (Aarons, 2004) (see section 3.14 for rationale and literature behind the choice of questions).

\subsection{Data Analysis}

This first section will describe and discuss the quantitative data analysis. The second section will describe and discuss the qualitative data analysis.

This section will describe the univariate, bivariate and regression analysis that I undertook on the quantitative components of the study. Data analysis for this part of the study was conducted in IBM SPSS version 22.0. For some of the variables I had to create 'dummy' variables. A dummy variable is an artificial variable created to represent an attribute with two or more distinct categories or levels. For example two dummy (yes / no) variables were coded for 'hub and spoke' and 'new enhanced community mental health team' with 'stand-alone' 
being the baseline category for the purposes of regression modelling because it was the most popular category.

\subsubsection{Missing Data}

Missing values arises in behavioural science data for many reasons. Unit nonresponse in sample surveys when some individuals are not contactable or refuse to respond, refuse to answer particular items in a questionnaire (Everett \& Howell, 2005, p.1234). Hence frequencies were computed one-way in order to identify where missing data were situated. Single imputation method was used for missing data. Frequencies were computed on EBPAS scale and the mode value was taken as the most popular. The pragmatic approach to handling missing data was to manually impute a missing value with the modal value of the variable whose value is missing.

Other missing data occurred where survey questions had 'other category please specify'. For example questions 5 (education), 6 (primary discipline) and 7 (service model).

\subsubsection{Exploratory Analysis}

One way frequencies were produced on the demographic categorical variables on the survey. This helped to operationalize the variables which confirmed the baseline category. The baseline category was the number of cases with the highest frequency number. Recoding was conducted on certain categorical variables allowing the combination of two or more categories which would tend to have lower frequencies / numbers of cases.

Bivariate analysis (two variables) were conducted next in order to uncover whether or not the two variables were related. Crosstabs were constructed and chi-squared tests were performed to explore associations and to compare the pattern of response between categories e.g. how the responses to age recode vary by years of experience working in mental health services. In the 'Findings' chapter four I present chi-squared and cross tabulations for selected pairs of variables. Chi-square was used to observe and test for difference in the pattern 
of response between two or more groups. An example of a cross tabulation of (reference to chapter 4) would permit an examination of how highest level of education differ in their response to the primary discipline. The corresponding test would allow a test of whether these differences observed in the crosstabs are statistically significant.

Two separate factor analytic procedures were conducted: confirmatory factor analysis and exploratory factor analysis.

\subsubsection{Statistical Modelling}

There are two basic types of factor analysis: exploratory factor analysis (EFA) and confirmatory factor analysis (CFA). CFA is form of multivariate statistical procedure that is used to test how well measured variables represent the number of constructs. In CFA, researchers can specify the number of factors required in the data and which measured variables is related to which latent variable.

EFA is most appropriately used when the links between the observed variables and their underlying factors are unknown or uncertain. Hence it is considered to be exploratory in the sense that the researcher has no prior knowledge that the observed variables do, indeed, measure the intended factors. In Everitt and Howell (2005) the authors stipulate that researchers use EFA to determine factor structure. In summarising the primary distinction between the two methodologies we can say that whereas EFA operates inductively in allowing the observed data to determine the underlying factor structure a posterior, CFA operates deductively in postulating the factor structure a priori (Bryant \& Yarnold, 1995).

Exploratory Factor Analysis was conducted using Principal Factor Analysis (PFA) in order to partition systematic and error variance in the solution (Fabrigar, Wegener, MacCallum \& Strahan, 1999; Nunnally \& Bernstein, 1994). The PFA suggested a four factor solution in accordance with examination of the scree plot, simple structure criteria, item total correlations, and Cronbach alpha 
analysis of internal consistency reliability. Assumption of normality was conducted on requirements scale, openness and EBPAS (see Appendix 3). A statistical model embodies a set of assumptions concerning the generation of the observed data. Models are relationships between variables.

Regression analyses were conducted in order to examine associations between the demographic variables and EBPAS subscales and total scores. Tables are presented in Appendix 13. Regression analyses were conducted in order to examine the association of EBPAS subscale (requirements and openness) and total scores with the demographic variables and organisational variables. For example a significant regression equation was found between EBPAS requirement scale and age recode variable. A significant regression equation was found on the EBPAS openness scale and psychiatry. More detailed descriptions of specific significant analysis are provided in the quantitative findings chapter four

Level of familiarity with the term "evidence based practice" or "empirically supported treatment" among early intervention practitioners was assessed. A mean score was computed in order to assess the degree which practitioners were familiar with the term EBP.

\subsubsection{Principle of Parsimony}

Mulaik (2005) states that parsimony or simplicity as a desirable feature of theories and models' was first popularized by the Franciscan William Ockham (1285-1347) using the principle 'Entities are not to be multiplied unnecessarily'. In other words, theoretical explanations should be as simple as possible, evoking the fewest explanatory entities as possible. In short the principle is one of simplicity in that we should not go looking for more complex explanations when a simple one will do. In practice it is essential that we have a philosophically justified method of choosing between explanations of our data. The parsimony principle is basic to all science and tells us to choose the simplest scientific explanation that fits the evidence (Sober, 1981). 


\subsubsection{Modelling: Building Processing and Selection}

In discussing model selection, Cortina (2005) explains that when a behavioural scientist statistically models relationships among a set of variables, the goal is to provide a meaningful and parsimonious explanation for those relationships, ultimately achieving a close approximation to reality. However, given the complexity inherent in social science data and the phenomenon they attempt to capture, there are typically multiple plausible explanations for any given set of observations. Even when one model fits well, other models with different substantive interpretations are virtually always possible.

One final caveat about model selection bears mention. Specifying a parsimonious model based on strong theory, testing it against viable alternatives, and evaluating it to have superior fit and interpretability, a researcher still cannot definitively claim to have captured 'Truth', or even to have identified the model that best approximates reality. Everett and Howell (2005) note that many models are always plausible, and selection of an excellent model could artifactually result from failure to consider every possible alternative (MacCallum, 1995). With behavioural processes being the complex, messy phenomena that they are, we can only aspire to represent them imperfectly in statistical models, rarely if ever knowing the 'true' model (Cortina, 2005).

The use of mechanically applied software-driven stepwise selection of variables is one approach to deciding what variables to include in a model when theoretical guidance is limited (Menard, 2010). Another approach sometimes used in research is, as a first step, to examine the bivariate relationship of each predictor to the dependent variable and then, in the second step, to include only those predictors satisfying some criterion (typically a statistically significant relationship with the dependent variable) in the model. Subsequent steps may further modify the model, possibly including the use of backward stepwise elimination to further reduce the model. 
Building process that includes key stages such as:

1. Each RV vs one EV main effects

2. Each RV vs main effects of two EVs

3. Each RV vs ME and interaction between two EVs

4. Stages 1 to 3 informed building of final or most parsimonious model

\section{Key stages 1 to 3}

The example below is from the quantitative chapter as applied to one of the dependent variables of interest, requirements scale.

The below is an example of the modelling building process. Simple and multiple regression analysis were conducted. The headings below outline the modelling building process.

Model 1: Gender main effect

The gender main effect was included in the regression analysis.

Model 2: Age main effect

The second model included the independent variable age with the requirement scale as the dependent variable.

Model 3: Age and Gender main effects

The third model includes the main effects of gender and age.

Model 4: Age and Gender main effects plus interactions

The fourth model includes the independent variable between age and gender.

Stages 1 to 3 were repeated for selected pairs of explanatory variables of primary interest and also for the other response variable of interest, namely EBPAS and openness. 


\section{$\underline{\text { Key stage } 4}$}

The results of stages 1 to 3 were used to inform the building of the final (or most parsimonious) model. For example, significant main effects and interactions were noted from stages 1 to 3 .

In order to arrive at the final main effects model all main effects whether significant or not in stages 1 to 3 were included initially. The final main effects model was determined using backward variable selection (see section 4.14.1).

To the final main effects model were added:

- Significant interactions from stage 3 (whilst ensuring that the respective main effects - whether significant or not - were previously included in the model).

- Any other interactions of substantive interest not previously noted as being significant at stage 3 .

Stepwise variable selection (a combination of both forward and backward methods) resulted in the final main effects plus interactions model (see section 4.15).

The goodness of fit for the final model was looked at for the EBPAS requirement scale. Then goodness of fit describes how well it fits the set of observations. The measure of goodness of fit typically summarizes the discrepancy between observed values and the values expected under the model in question.

\subsection{Qualitative Data Analysis}

Eighteen complete interview transcripts were audio-recorded with an Olympus digital voice recorder WS-832. The transcripts included pauses, laughter, each participant's manner, crosstalk, style and speech for example "Oh, no it has been about - gosh". The recordings consisted of almost 8 hours of talk with 55,672 words of transcribed talk available for analysis. 


\subsubsection{Thematic Analysis}

A thematic approach to analysis was adopted for all qualitative data (Braun \& Clarke, 2006). Braun and Clarke argue that thematic analysis is a method for identifying, analysing and reporting patterns (themes) within data. The six steps are set out below.

\section{Phases of thematic analysis:}

1) Familiarizing yourself with your data (transcribing data, reading and rereading the data, and noting down initial ideas)

2) Generating initial codes (coding interesting features of the data in a systematic fashion across the entire data set, collating data relevant to each code).

3) Searching for themes (collating codes into potential themes, gathering all data relevant to each potential theme)

4) Reviewing themes (checking if the themes work in relation to the coded extracts (Level 1) and the entire data set (level 2), generating a thematic 'map' of the analysis).

5) Defining and naming themes (ongoing analysis to refine the specifics of each theme, and the overall story the analysis tells, generating clear definitions and names for each theme.

6) Producing the report: (selection of vivid, compelling extract examples, final analysis of selected extracts, relating back of the analysis to the research questions and literature, producing a scholarly report of the analysis).

The semi-structured research interview was organised into three sections $A, B$ and $C$. Section A was sustaining practices in teams, section $B$ was barriers and facilitators of evidence based practices and section $\mathrm{C}$ was adherence to policies and protocols.

I first read the eighteen transcripts and then re-read them line by line horizontally and vertically. We are reminded that the analysis of qualitative data begins with the immersion in the data and as noted by Braun and Clarke this 
phase is to become intimately familiar with ones data set content and to begin to notice things that might be relevant to the research questions. The second phase was to read the data again and generate initial codes throughout the whole data set. Codes are conceptualized variously as the "names or symbols used to stand for a group or similar terms, ideas, or phenomena" that you notice in your data (LeCompte \& Schensul, 1999, p. 55).

Coding is a process of identifying aspects of the data that relate to the researchers research questions. Braun and Clarke (2013) describe two approaches to coding in pattern-based forms of qualitative analysis which they call selective coding and complete coding. For the purposes of my qualitative analysis I used complete coding. In complete coding the researcher aims to identify anything and everything of interest or relevance to answering the research questions within the entire dataset. This means that rather than selecting out a particular corpus of instances which then is analysed the researcher codes all the data that's relevant to the research questions. It's only later in the analytic process that one becomes more selective. In complete coding Braun and Clarke (2013) explain that codes identify and provide a label for a feature of the data that is potentially pertinent for answering the research questions. A code is a word or brief phrase that captures the essence of why you think a particular bit of data may be useful. When coding in this study I used words or a brief phrase which captured the essence of why I thought a particular bit of data may be useful in relation to my research questions. I coded chucks of data in as many ways as needed throughout the whole data corpus. Braun and Clarke (2013) recommend to over code rather than under code as it is much easier to discard codes than go back to the data and recode it all later. In Table 1 below I provide an example of a quote extract along with the code, category, theme and theme definition from the question asked about how practitioners could help me understand the sustaining or not sustaining of evidence based practices at their organisation. 
Table 1: Example of coding from research (See Appendix 8 for theme 1 and then

\begin{tabular}{|l|l|l|l|l|}
\hline \multicolumn{1}{|c|}{ Quote } & \multicolumn{1}{c|}{ Code } & \multicolumn{1}{c|}{ Category } & \multicolumn{1}{c|}{ Theme 1 } & \multicolumn{1}{c|}{ Theme c c } \\
\hline $\begin{array}{l}\text { "I think secondly, fortunately } \\
\text { the robust evidence base for } \\
\text { early intervention and that has } \\
\text { finally kind of filtered down into } \\
\text { the politics and Department of } \\
\text { Health; so early intervention is } \\
\text { a by-word now used within } \\
\text { various political manifestos. Not } \\
\text { necessarily that they } \\
\text { completely understand what it } \\
\text { means and what the evidence } \\
\text { base is but, at least there does } \\
\text { appear to be an understanding } \\
\text { there". (Richard) }\end{array}$ & & $\begin{array}{l}\text { What constitutes } \\
\text { EBP in relation } \\
\text { to EIP? }\end{array}$ & $\begin{array}{l}\text { Sustaining } \\
\text { Evidence } \\
\text { Based } \\
\text { Practices }\end{array}$ & $\begin{array}{l}\text { Robust effectiven } \\
\text { that EIP; evidenc } \\
\text { how this fits or do } \\
\text { evidence based p }\end{array}$ \\
\end{tabular}




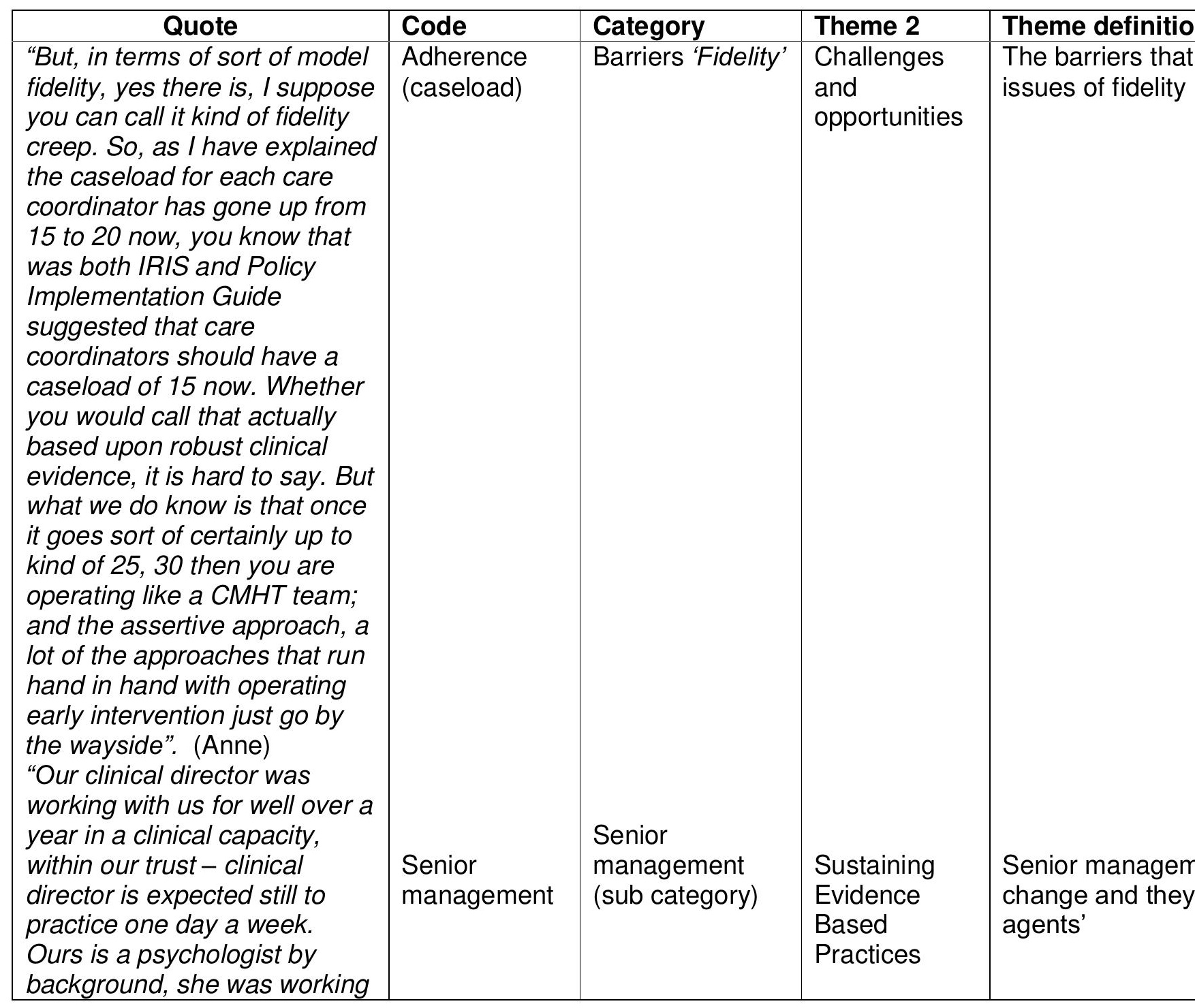


with our service users and within our team. I think that helped enormously for her to understand what we do, and the value of it and the demands and challenges". (Martin) 


\subsection{Conclusion}

In this chapter the aims and research questions of the study have been described, the research design has been presented and the reasons why the approach was considered suitable for this particular research question have been explained.

The various elements of data collection process have been described. These include quantitative methods involving the use of an online survey, and the use of qualitative methods that involved semi-structured interviews with early intervention practitioners within NHS Trusts in England and Wales.

Validity and rigour of the project have been described. Ethical issues have been discussed, and anonymity and confidentiality.

In the following pages I present analysis of the data collected for this study. In Chapter 4 I present the quantitative results from the survey of early intervention practitioners using the EBPAS and show that EBPAS requirement and openness scales are strong predictors of attitudes. In chapter 5 I present my analysis of the qualitative findings and show how practitioners talk about their experiences of delivering evidence based early intervention services. 


\section{CHAPTER 4: Quantitative Findings from the Survey}

\subsection{Introduction}

In this chapter I address the research question one, 'what are the attitudes of early intervention lead practitioners in relation to evidence based practice?' The purpose of this chapter is to show the significant findings from my analysis of demographic information and Evidence Based Attitudinal Scale (EBPAS) data collected from the phase 1 survey. In this analysis I will show descriptive, bivariate and statistical modelling such as regression analysis. This analysis forms the first part of the explanatory sequential mixed methods approach used to assess the implementation of EBPs in mental health services in England and Wales. My intention was to achieve a total population sample of practitioners identified through the IRIS and the 1000 Lives networks.

\subsection{Data Description / Exploratory Analysis}

Research was conducted to examine implementation of early intervention practitioner attitudes to evidence based practice in England and Wales. The early intervention practitioners were accessed via two programmes in England and Wales. Firstly, the early intervention Initiative to Reduce the Impact of Schizophrenia (IRIS) in England and secondly, the 1000 lives plus programme set up in Wales. Early intervention practitioners who were invited to take part worked within early intervention teams in NHS trusts and health boards.

\subsection{Selection Criteria of Participants}

A total population sample included health care organisations comprising of staff who worked in an early intervention mutli-disciplinary teams in mental health which comprised of hybrid managers (managers that are in clinical role) these were community psychiatric nurses (CPNs) psychiatrist, social workers, occupational therapists, psychologists. 


\subsection{Sample Description}

There were 37 females and 33 males who responded to the survey for this study. The majority of the participants fell into the age category of 35 to 44 years $(n=33)$.

Table 2: Age descriptives

\begin{tabular}{lc}
\hline \multicolumn{1}{c}{ Age categories } & Sum \\
\hline $18-24$ years & 1 \\
$25-34$ years & 4 \\
$35-44$ years & 33 \\
$45-54$ years & 28 \\
$55-64$ years & 4 \\
$65-74$ years & 0 \\
75 years or & 0 \\
older \\
\hline \multicolumn{2}{c}{ Note: $N=70$}
\end{tabular}

\subsection{Descriptive Analysis Responses to the Survey Questions}

In table 4 below show the descriptive statistics: frequency, percentage which were computed on the demographic variables and the Evidence Based Practice Attitudinal Scale (Aarons, 2004). The re-coding is explained in the methods chapter under the analysis section 3.20.2. An example is provided below in Table 3.

\subsection{An Example of Data Processing: re-coding Primary Discipline}

Table 3 below shows the primary discipline variable had six categories, with 'other' category for the participant to specify. Due to low numbers of participants in primary disciplines such as 'social work' $(n=3)$, 'occupational therapy' $(n=4)$ and 'other' ( $n=2)$ a re-code was made to combine counselling and psychotherapy. This new category was called 'therapists / social work'. Table 4 shows the final recode of the four dummy variables which were created for the purpose of modelling. These were: 'nursing', 'psychology', 'psychiatry' and 
'therapy / social work'. Other and the missing value were subsumed into the 'therapists / social work'. See Appendix 10 for all data processing recoding.

Table 3: Original variable - primary discipline

\begin{tabular}{lcc}
\hline \multicolumn{1}{c}{ Primary discipline } & Frequency & Percent \\
\hline Psychiatry & 12 & 17.1 \\
Nursing & 35 & 50.0 \\
Social Work & 3 & 4.3 \\
Occupational Therapy & 4 & 5.7 \\
Psychology & 13 & 18.6 \\
Other & 2 & 2.9 \\
Missing & 1 & 1.4 \\
Total & 70 & 100 \\
\hline
\end{tabular}

Table 4: Final recode of the primary discipline variable

\begin{tabular}{lcc}
\hline \multicolumn{1}{c}{ Primary discipline } & Frequency & Percent \\
\hline Nursing & 35 & 50.0 \\
Psychology & 13 & 18.6 \\
Psychiatry & 12 & 17.1 \\
Therapists / Social work & 10 & 14.3 \\
Total & 70 & 100 \\
\hline
\end{tabular}

\subsection{Summary of Demographic Variables Used in the Survey}

Participant variables used in the survey by demography, see Appendix 11 .

There were 35 participants (50\%) who worked in nursing, 13 participants (18.6\%) who worked as psychologists, 12 participants (17.1\%) who worked in psychiatry and 10 participants (14.3\%) who worked as therapists or social work.

The variability of adherence to Mental Health Policy Implementation Guidelines (MHPIG) between England and Wales was substantial 55 (78.6\%). Responses varied in accordance to years of experience that practitioners had worked within mental health services zero to ten years $(n=10,14.35 \%)$ from eleven to fifteen years $(n=20,28.6 \%)$ and sixteen plus years $(n=40,57.1 \%)$. The lengths of time that early intervention teams had been established were evenly spread. Respondents stated one to five years $(n=15,21.4 \%)$, six to nine years $(n=19$, $27.1 \%)$, ten years $(n=17,24.3 \%)$ and eleven years or over $(n=19,27.1 \%)$. 


\subsection{Descriptives on the Evidence Based Attitudinal Scale (EBPAS)}

Descriptive statistics included: frequency, percentage, mean and standard deviation that were computed on EBPAS. The four scales consisted of: requirements, appeal, openness and divergence which are presented in Appendix 12. Respondents were asked about their views on using new types of therapy interventions or treatments from using the Survey Monkey, indicating the extent to which they agreed with each item; from not at all (0) to a very great extent (4). The divergence scale is presented below in sets of pie charts. See the EBPAS scale description below on the divergence scale. The first pie chart is the divergence scale. The second pie chart is the divergence scale reversed. 
When respondents were asked, 'I know better than academic researchers how to care for my clien not at all, $22(31.4 \%)$ stated to a slight extent and $20(28.6 \%)$ respondents stated to a moderate ext

\section{Figures 5}
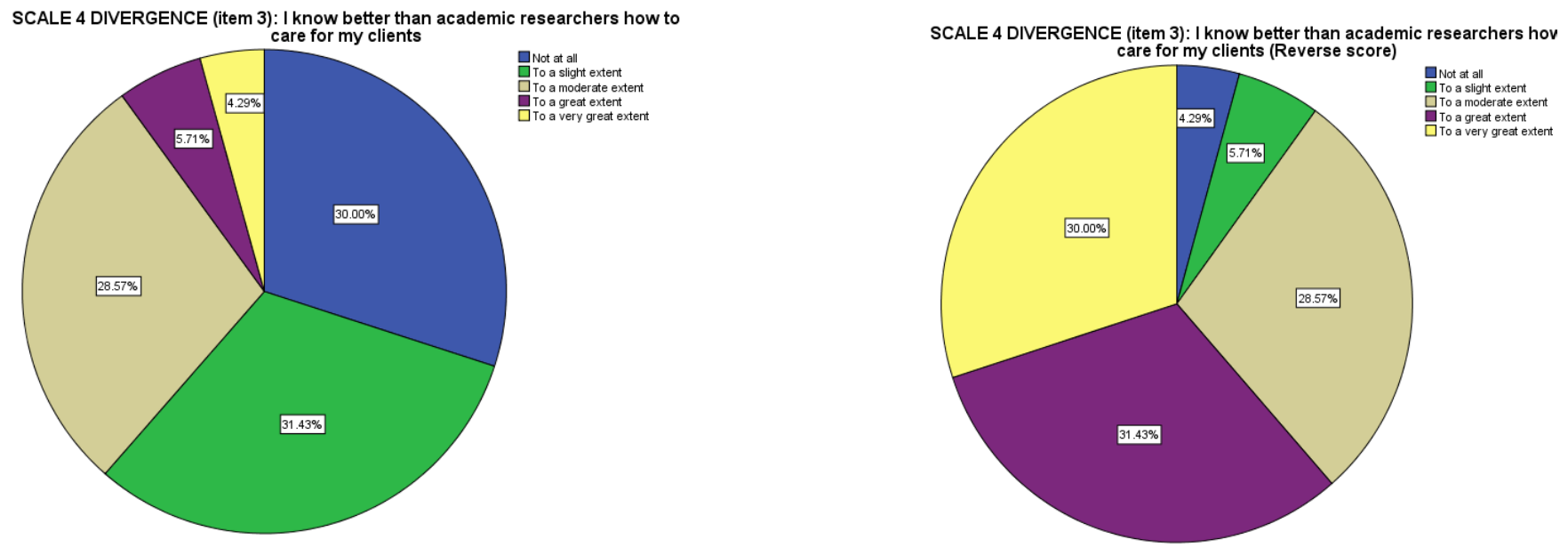
When respondents were asked, 'clinical experience is more important than using manualised thera $(28.6 \%)$ stated to a slight extent with 32 respondents $(45.7 \%)$ stating to a moderate extent.

\section{Figures 6}
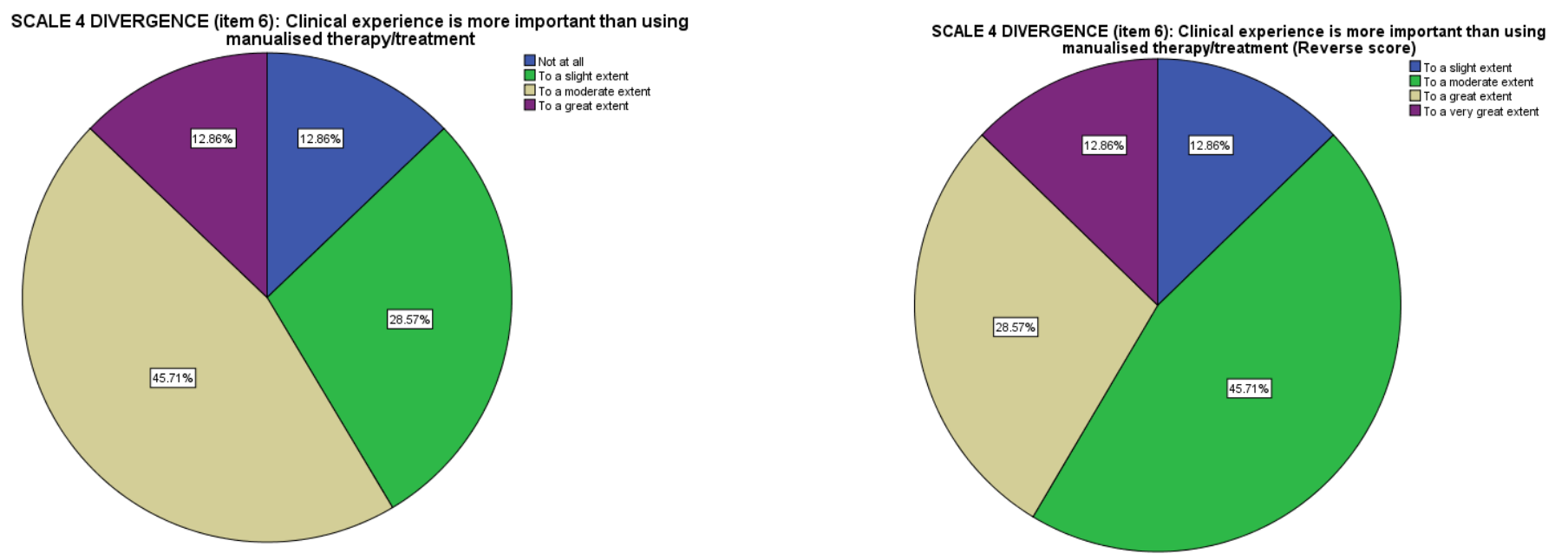
When respondents were asked, 'Research based treatments / interventions are not clinically usefu stated to a slight extent (17.1\%).

\section{Figures 7}
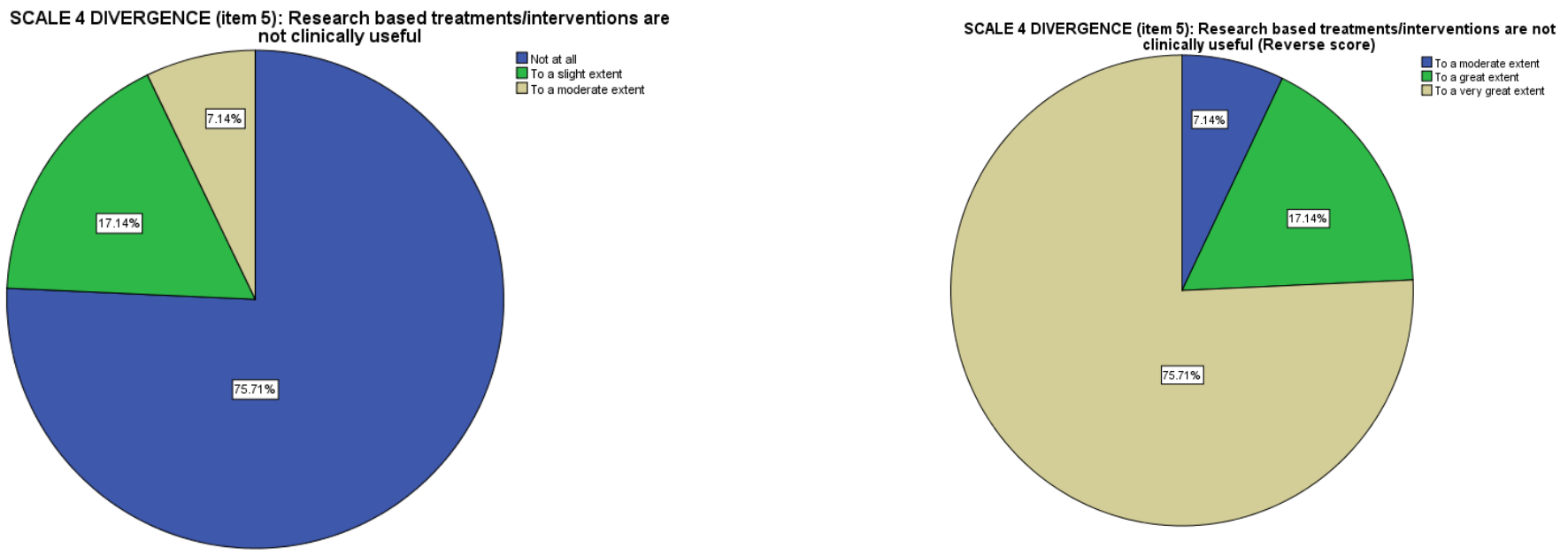
When respondents were asked, 'I would not use manualised therapy / interventions' 55 (78.6\%) sta stating to a slight extent and $4(5.7 \%)$ stating to a moderate extent.

\section{Figures 8}
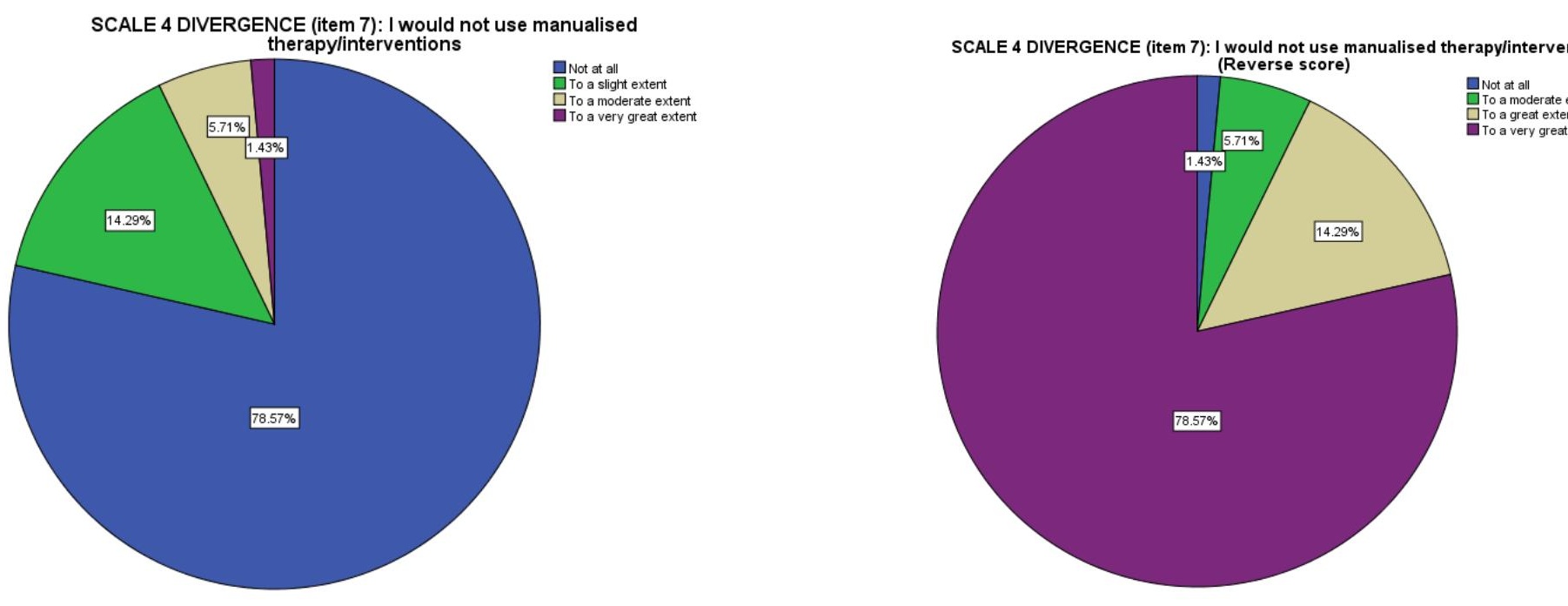


\subsection{Bivariate Analysis: Examining the Relationship Between two Categorical Variables}

Bivariate analyses were conducted in the following sections selected pair of demographic variables. The test statistic chi-squared and corresponding ' $p$ ' values for the variables across categories. Clustered bar charts were used as a graphical display of relationships between pairs of categorical variables.

\subsubsection{Age with how many years' experience you have been working in mental health services}

Figure 9 is a clustered bar chart showing the age and relationship between years of experience in the services.

The respondents within the 44 years or less are, $28.9 \%$, who had $\geq 16$ year's experience. Within the 45 years experience category, this rose to $90.6 \%$ with participants also having $\geq 16$ years experience in the service. The respondents within 44 years or less had 11 to 15 years experience with a percentage reduced to $44.7 \%$.

A smaller percentage within the 45 year category, $9.4 \%$ also had 11 to 15 years work experience. Again, within the 44 year or less category, from zero to ten years the percentage was $26.32 \%$.

The association between age and years of experience working in mental health services is highly significant at the $0.1 \%$ (chi-squared $=27.58, p<0.0005$ ). 


\section{Figure 9}

A clustered bar chart showing age with years experience of working in mental health services

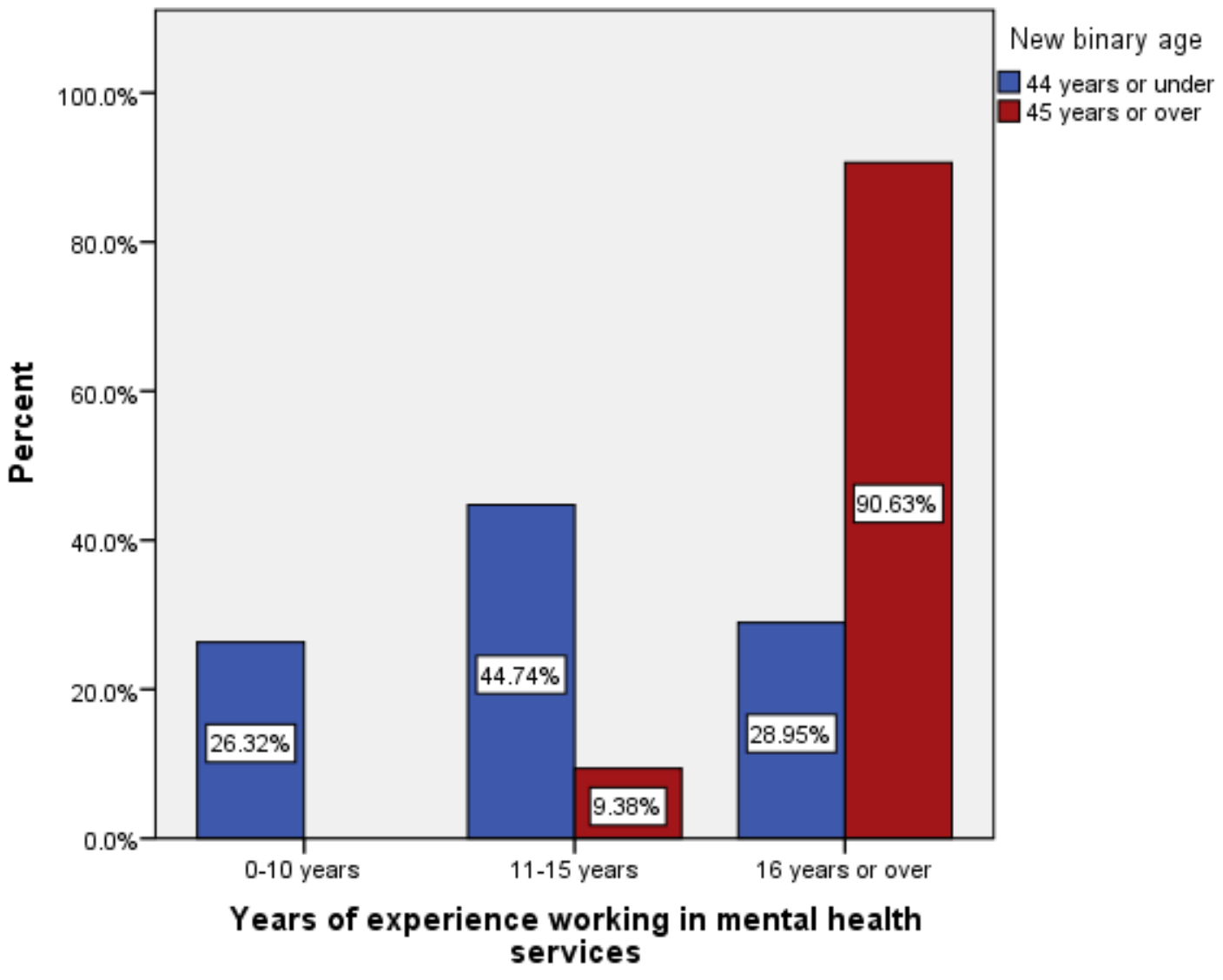




\subsubsection{Highest Level of Education with Primary Discipline}

Figure 10 shows a clustered bar chart showing highest level of education within the primary disciplines. The educational level question was a multiple response asking the participant to tick all that apply. The responses were re-coded into the highest level of education. Professional qualification is the highest within nursing discipline at, $(88.9 \%)$, compared to psychiatry at (8.3\%). Degree is highest within the nursing discipline at (70.8\%), compared to other disciplines. Participants that were educated to $\mathrm{PhD} /$ Doctorate or fellowship were present in disciplines such as psychology (52.94\%), psychiatry (35.29\%) and social work $(11.76 \%)$. The association between highest level of education and with primary discipline is highly significant at the $0.1 \%$ level (chi-squared $=35.55, p=$ $<0.0005)$. However, this result should be treated with caution due to the sparse nature of the cross-tabulation (13 cells out of 16 (81.3\%) have an expected count less than 5).

\section{Figure 10}

\section{A clustered bar chart showing the highest level of education within each primary} discipline

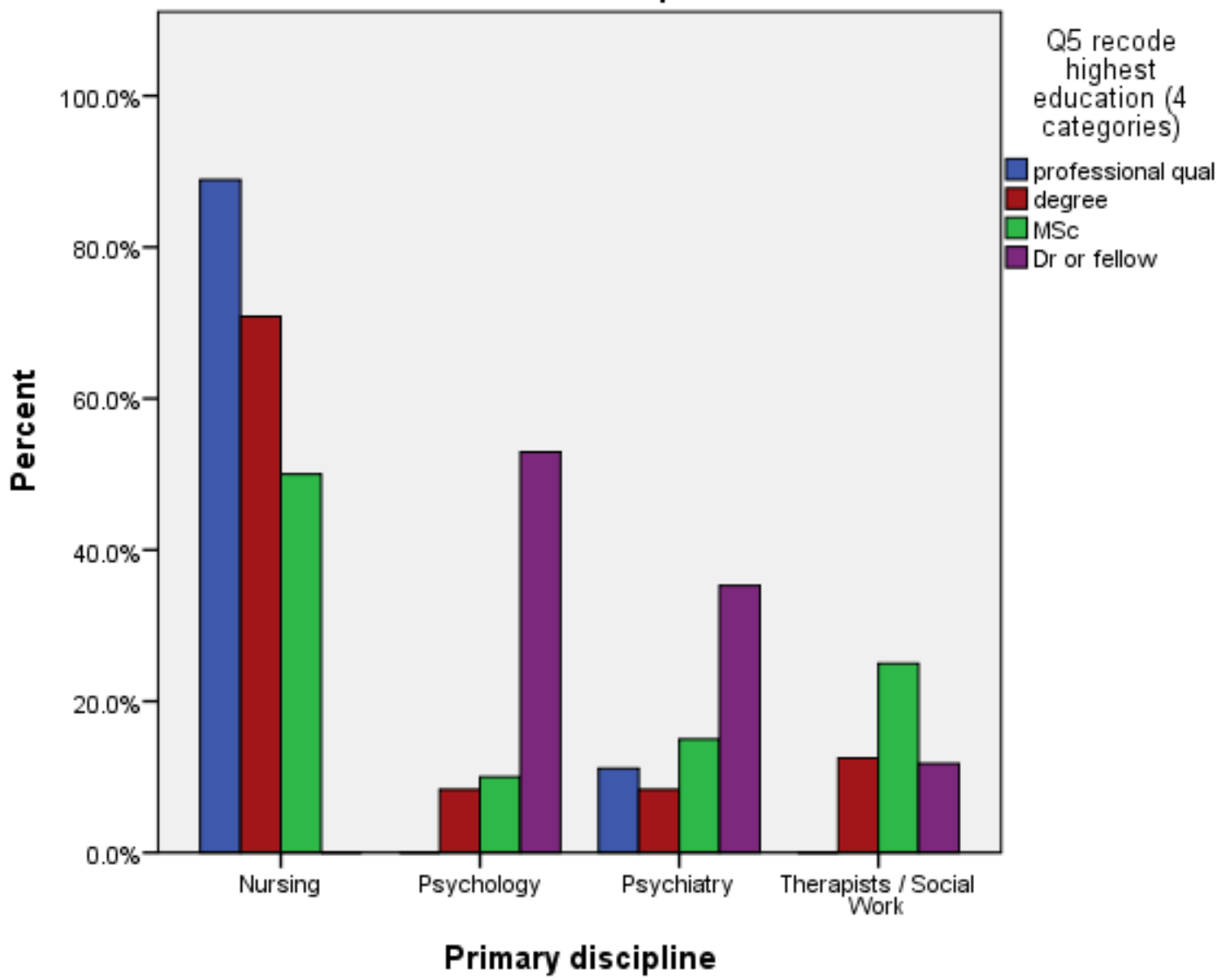




\subsubsection{Highest Level of Education with Types of Services Provided by Service Model: Inpatient}

Figure 11 shows a clustered bar chart with highest level of education by service model inpatient. The bar chart shows that those respondents who did not provide inpatient as part of their service model $100 \%$ of these had a professional qualification; $58.3 \%$ had a degree; $95 \%$ had a Masters degree and $88.2 \%$ had a doctor or fellowship.

The association between highest level of education with inpatient service is approaching significance at $0.1 \%$ level (chi-squared $=13.47 .55, \mathrm{p}=<0.0005$ ). However, this result should be treated with caution due to the sparse nature of the cross-tabulation ( 4 cells out of $8(50 \%)$ have an expected count less than 5 ).

\section{Figure 11}

A clustered bar chart showing the highest level of education provided by service model (inpatient)

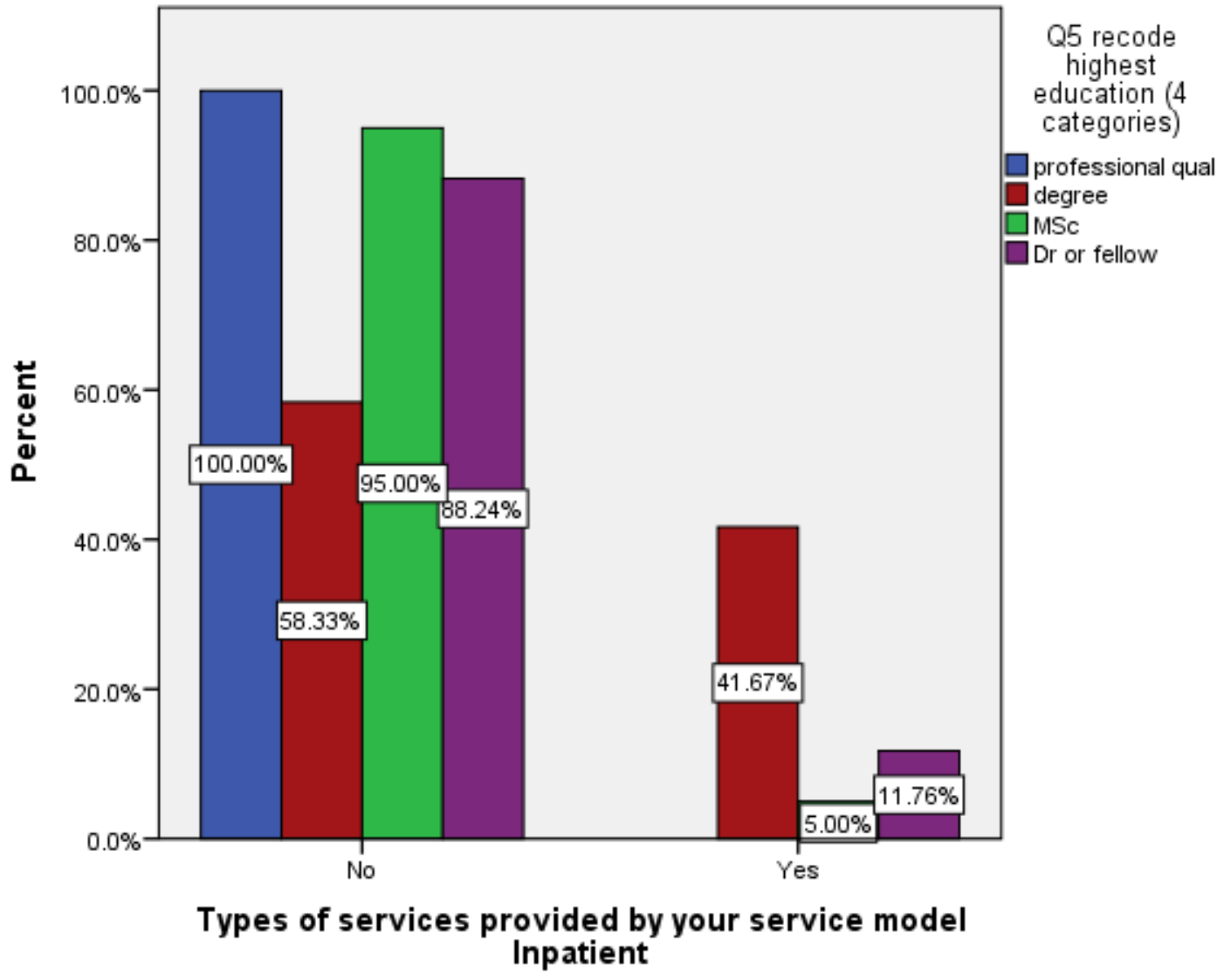




\subsubsection{Types of Services Provided by your Service Model: Inpatient with Outpatient}

Table 6 shows a cross tabulation of row percentages of respondents provided by service model inpatient and outpatient. Of the respondents who did not provide inpatient services, $17.5 \%$ did provide outpatient services. Of the respondents that provided inpatient services, $84.6 \%$ did provide outpatient services. The association between types of services provided by the respondents' service models between inpatient and outpatient is highly significant at the $0.1 \%$ level (chi-squared $=22.67, p<0.0005$ ).

Table 5: Cross tabulation showing the type of service provided by service model inpatient and outpatient

\begin{tabular}{lcccc}
\hline \multirow{3}{*}{ Inpatient } & \multicolumn{3}{c}{ Outpatient } & Totals \\
\cline { 2 - 5 } & & No & Yes & \\
\cline { 2 - 5 } & No & $82.5 \%$ & $17.5 \%$ & $100 \%$ \\
& Yes & $15.4 \%$ & $84.6 \%$ & $100 \%$ \\
\hline
\end{tabular}

\subsubsection{Types of Services Provided by your Service Model Inpatient with Day Treatment}

Table 6 shows a cross tabulation of the percentage of respondents provided by service model inpatient and day treatment. Of the respondents who did not provide inpatient services, $8.8 \%$ did provide day treatment services. Of the respondents that provided inpatient services, $38.5 \%$ did provide day treatment services. The association between types of services provided by the service model between, inpatient and day treatment is highly significant at the $0.1 \%$ level (chi-squared $=7.620, p<0.0005)$.

Table 6: Cross tabulation showing the types of services provided by your service model inpatient with day treatment

\begin{tabular}{ccccc}
\hline \multirow{3}{*}{ Inpatient } & \multicolumn{4}{c}{ Day Treatment } \\
nyyyy & No & Yes & Totals \\
\cline { 2 - 5 } & No & $91.2 \%$ & $8.8 \%$ & $100 \%$ \\
& Yes & $61.5 \%$ & $38.5 \%$ & $100 \%$ \\
\hline
\end{tabular}




\subsubsection{Does your Organisation Adhere to the following Policies:}

Welsh Psychological Therapies with Practice Policy Mental

\section{Health Wales's Measure}

Figure 12 shows a clustered bar chart showing adherence to the Welsh psychological therapies policy with the Wales mental health measure policy. Of those respondents who did not adhere to the Welsh psychological therapies, $14.3 \%$ did adhere to the Wales Mental health measure. Of the respondents that did adhere to the Welsh psychological therapies, all did adhere to the Wales mental health measure. The association between whether the NHS or trust adheres to policies (Welsh psychological therapies) with Wales Mental Health Measure is highly significant at the $0.1 \%$ level (chi-squared $=26.25, p<$ 0.0005).

Figure 12

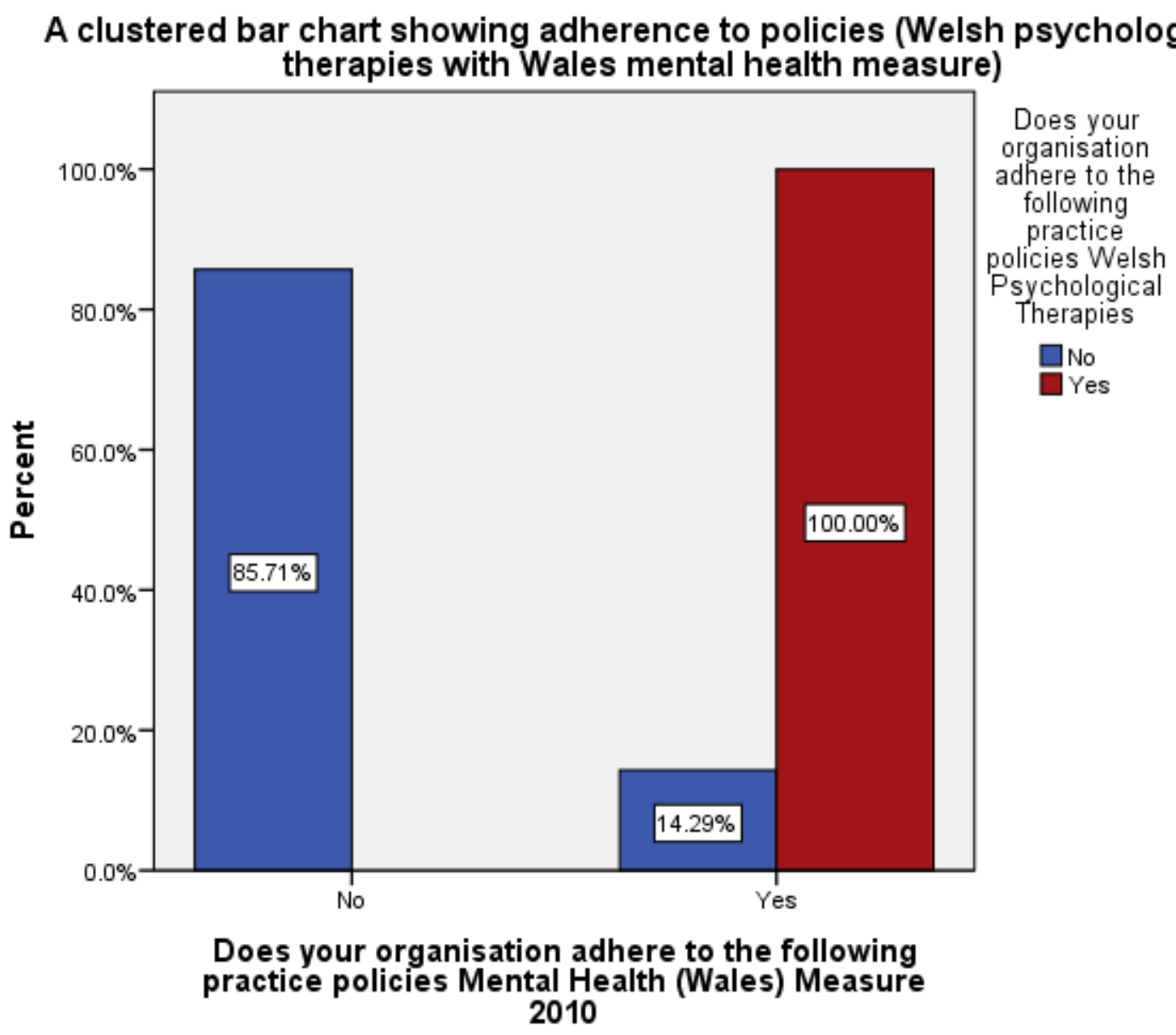




\subsubsection{How long has your Early Intervention Team been Established with does your Organisation Adhere to the Following Policies: Wales's Mental Health Measure}

Figure 13 shows a clustered bar chart which shows how long early intervention team has been established with adherence to the Wales mental health measure.

The bar chart shows that those teams established 11 years and over, $31.5 \%$ of the respondents did not adhere to the Wales mental health policy. The same applied to those teams established 10 years, $31.5 \%$ of the respondents did not adhere to the Wales mental health policy.

Those early intervention teams established 1 to 5 years $62.5 \%$ of the respondents said that their organisation did adhere to the Wales mental health measure policy. Early intervention teams that had been established 6 to 9 years, $25 \%$ of the respondents said that their organisation did adhere to the Wales mental health measure.

The association between whether the NHS or trust adheres to policies (Wales Mental Health Measure) and the establishment of an early intervention team is highly significant (chi-squared $=23.03, p<0.0005$ ). However, this result should be treated with caution due to the sparse nature of the cross-tabulation (4 cells out of $8(50 \%)$ have an expected count less than 5). 
Figure 13

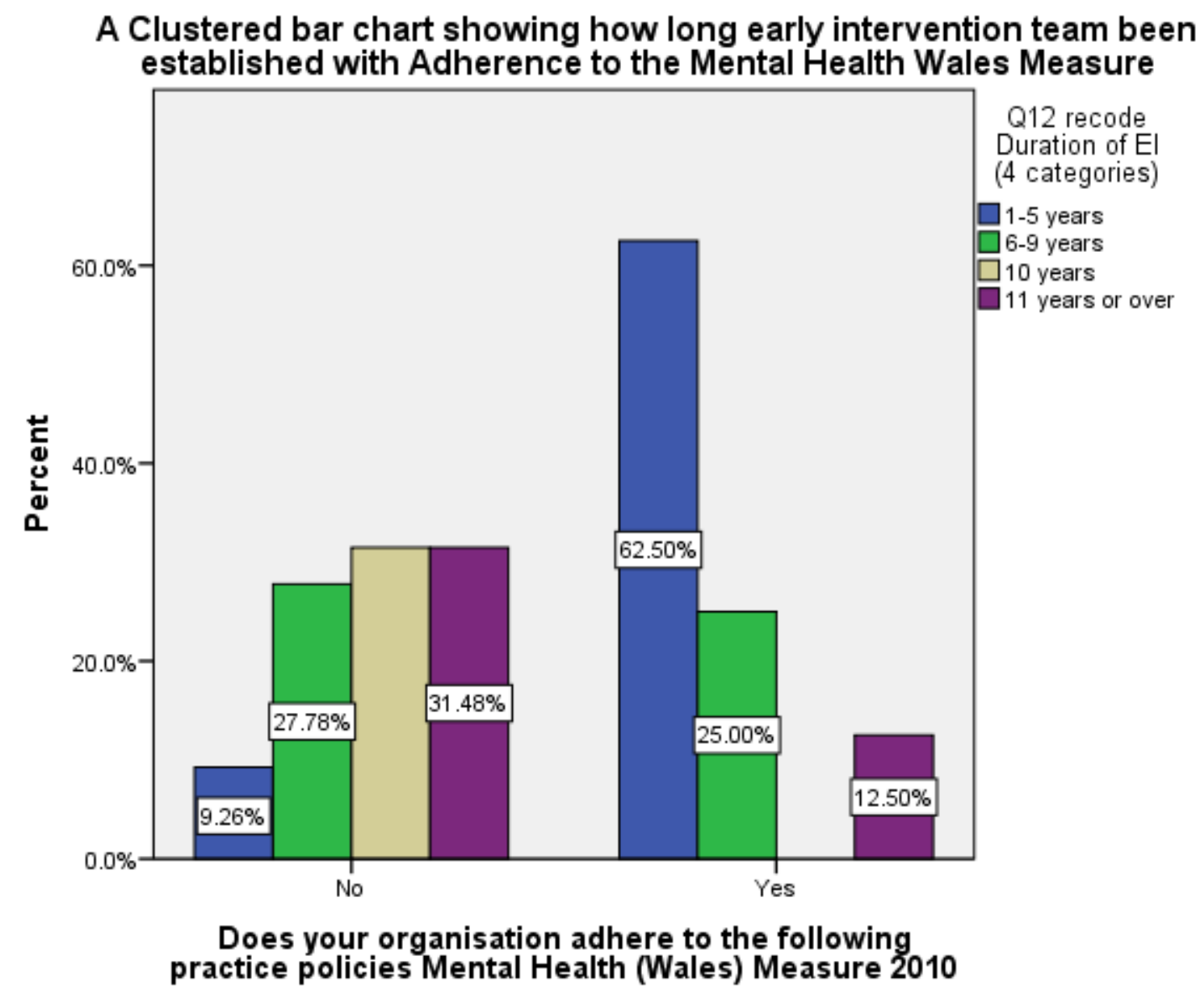




\subsection{Level of Familiarity with Evidence Based Practice and Empirical Supported Treatments}

Level of familiarity with EBP was assessed with early intervention practitioner leads. Early intervention practitioner leads were asked the degree to which they were familiar with the terms "evidence based practice" and "empirically supported treatment" (Aarons, 2004). A mean score was computed in order to assess the degree to which practitioners were familiar with the term 'Evidence based Practice'. The figure 14 shows the breakdown of percentages of how early intervention practitioners were familiar with the terms "evidence-based practice" and "empirically supported treatments". Overall the mean familiarity rating was 2.97 (SD = 0.722). One practitioner reported "not at all familiar" $(1.4 \%)$; thirteen practitioners reported "to a moderate extent" (18.6\%); forty-two practitioners reported "to a great extent" $(60 \%)$ and fourteen practitioners reported "to a very great extent" $(20 \%)$.

\section{Figure 14: A Pie Chart showing Familiarity of EBP and EST}

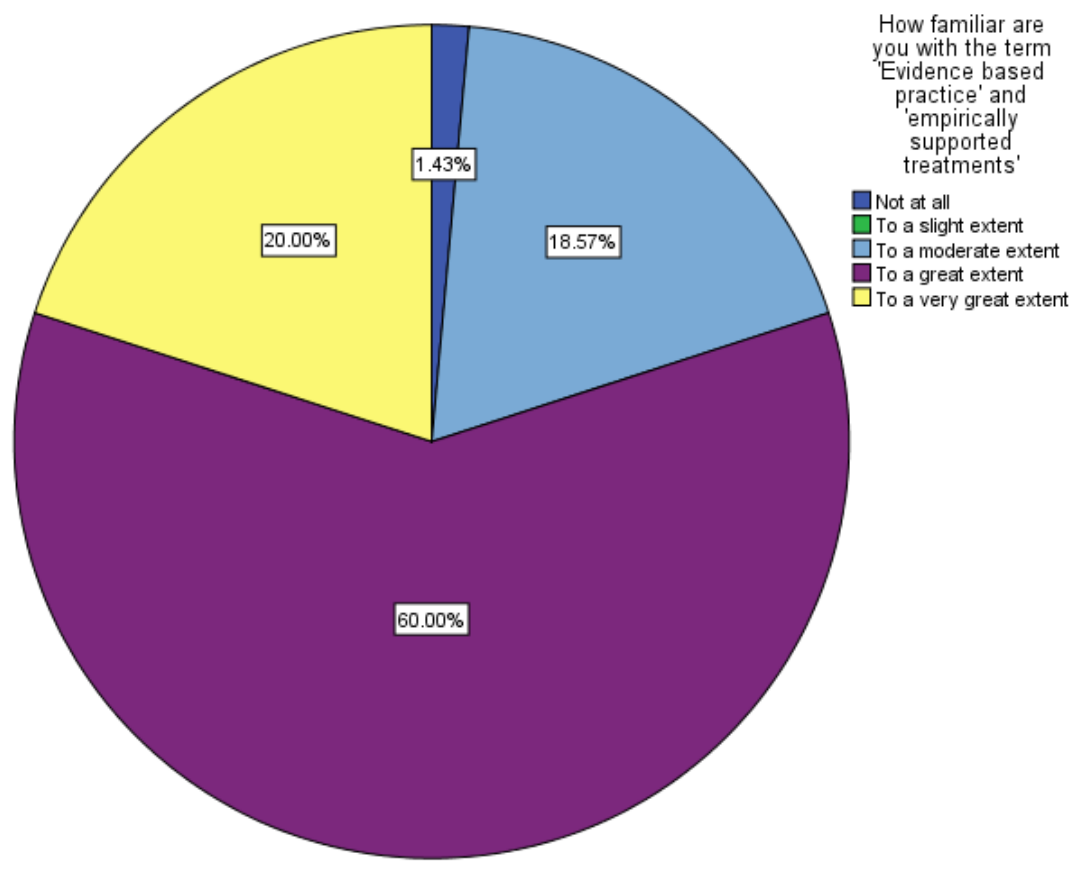


Fifteen of the original eighteen items were retained and the Principal Factor Analysis model accounted for $81 \%$ of the variance in the data. Table 8 shows overall means, standard deviations, item total correlations, eigenvalues, internal consistency reliabilities, and item loadings for each of the scales. Cronbach's alphas ranged from .89 to .47 with an overall scale alpha of .81 . The factors represented four subscales of attitudes towards adoption of EBPs.

Appeal (four items; $\alpha=.77$ ) is the extent to which the provider would adopt a new practice if it is intuitively appealing, makes sense, could be used correctly, or is being used by colleagues who are happy with it. Requirements (three items; $\alpha=.89$ ) is the extent to which the provider would adopt a new practice if it is required by the organisation, supervisor or country. Openness (four items; $\alpha$ $=.83$ ) is the extent to which the provider is generally open to trying new interventions and would be willing to try or use new types of therapy. Divergence (four items; $\alpha=.47$ ) is the extent to which the provider perceives research-based interventions as not clinically useful and less important than clinical experience.

The table 7 shows the factors which represent the four subscales of attitudes towards adoption of EBP in Aarons (2004) study and in the retrospect study. The alphas show a good range of internal and level of consistency between studies.

Table 7: Comparison of alphas

\begin{tabular}{lccccc}
\hline & $\begin{array}{c}\text { Requirements } \\
\alpha\end{array}$ & $\begin{array}{c}\text { Appeal } \\
\alpha\end{array}$ & $\begin{array}{c}\text { Openness } \\
\alpha\end{array}$ & $\begin{array}{c}\text { Divergence } \\
\alpha\end{array}$ & $\begin{array}{c}\text { Overall } \\
\alpha\end{array}$ \\
\hline $\begin{array}{l}\text { Aarons } \\
\text { (2004) study }\end{array}$ & .90 & .80 & .78 & .59 & .77 \\
$\begin{array}{l}\text { White (2015) } \\
\text { study }\end{array}$ & .89 & .77 & .83 & .47 & .81 \\
\hline Note: $\alpha=$ alpha & & & & &
\end{tabular}


Table 8: Overall means, standard deviations, item total correlations, eigenvalues, internal and item loadings for each of the scales for EBPAS

\begin{tabular}{|c|c|c|c|c|c|c|}
\hline EBPAS subscales and total & Mean & SD & $\begin{array}{l}\text { Item total } \\
\text { correlation }\end{array}$ & EV & $\alpha$ & Scale 1 \\
\hline 1. Requirements & 2.09 & .022 & & 4.612 & .897 & \\
\hline Organisation required & 2.10 & .965 & .574 & & & .916 \\
\hline Supervisor required & 2.09 & .944 & .660 & & & .816 \\
\hline Country required & 2.07 & 1.054 & .610 & & & .872 \\
\hline 2. Appeal & 2.93 & .243 & & 1.799 & .771 & \\
\hline Makes sense & 3.06 & .883 & .574 & & & \\
\hline Intuitively appealing & 2.79 & .866 & .541 & & & \\
\hline Get enough training to use & 3.20 & .734 & .465 & & & \\
\hline Colleagues happy intervention & 2.67 & .717 & .521 & & & .451 \\
\hline 3. Openness & 2.64 & .158 & & 1.138 & .839 & \\
\hline Will follow a treatment manual & 2.51 & .944 & .516 & & & \\
\hline Like new therapy types & 2.73 & .760 & .522 & & & \\
\hline Therapy developed by researchers & 2.81 & .687 & .500 & & & \\
\hline Therapy different than usual & 2.50 & .897 & .526 & & & \\
\hline 4. Divergence & 3.13 & .643 & & .785 & .472 & \\
\hline $\begin{array}{l}\text { Research based treatments not } \\
\text { useful }\end{array}$ & 3.69 & .603 & .213 & & & \\
\hline Will not use manualised therapy & 3.66 & .778 & .257 & & & \\
\hline Clinical exp more important & 2.41 & .876 & 181 & & & \\
\hline Know better than researchers & 2.77 & 1.079 & -.117 & & & \\
\hline EBPAS total & 2.736 & .504 & & & .812 & \\
\hline
\end{tabular}




\subsection{Statistical Modelling}

Regression, like correlation coefficient, numerically describes important features of a scattergram relating two variables. Upon inspection of table 8 there are strong correlations on the EBPAS requirements and openness scales.

A series of three separate linear regression analysis were computed. The first regression analysis was conducted on the EBPAS 'requirement' scale as the dependent variable and the demographic variables were entered as independent predictor variables. The independent variables were entered into the equation depending on whether the demographic variables had singular response or multiple responses, where variables had multiple responses the baseline category was excluded from the analysis as the researcher made conservative decision in that this was the highest out of the reference group.

The second regression analysis was conducted on the EBPAS 'openness' scale as the dependent variable and the demographic variables were entered as above. The third regression analysis was conducted on the EBPAS means total as the dependent variable and the demographic variables were entered as above. The significant results are presented below. All results are presented in Appendix 13.

\subsection{Regression Analysis}

Regression analysis was conducted in order to examine the association of EBPAS subscale (requirements and openness) and total scores with the demographic variables and organisational variables. The significant results are presented below.

\subsubsection{Factor 1 of Analysis 3, EBPAS Scale Requirement}

Table 9 below shows the results of one simple linear regression analysis. This was calculated to predict EBPAS requirement scale based on age variable. A significant regression equation was found $\left(F(1,68)=3.340, p=0.072, R^{2}\right.$ of $0.047)$. The results indicate that older respondents were scoring lower on average than the younger respondents subsumed in the baseline. The older respondents were less likely to adopt a therapy or intervention if they received 
training if it was required by their supervisor, organisation and country than the younger respondents in the baseline category.

Table 9: Factor 1 of analysis 3, EBPAS scale Requirement with age

\begin{tabular}{ccccc}
\hline Model & $\mathrm{B}$ & $\mathrm{SE}$ & $\mathrm{t}$ & $\mathrm{Sig}$ \\
\hline Baseline (younger) & 0.188 & 0.153 & 1.236 & 0.221 \\
Age (older) & -0.412 & 0.226 & -1.828 & 0.072 \\
\hline
\end{tabular}

Table 10 below shows the results of one simple linear regression analysis. This was calculated to predict EBPAS requirement scale based on the organisation adherence to Policy Implementation Guidance. A significant regression equation was found $\left(F(1,68)=7.216, p=0.009, R^{2}\right.$ of 0.310$)$. These results indicate that those respondents whose organisations adhere to the PIG policy, on average are scoring significantly higher on the requirements scale than those who don't adhere who are scoring significantly lower in the baseline. 
Table 10: Factor 1 of analysis 3, EBPAS scale Requirement with Organisation adheres to Policy Implementation Guidance (PIG)

\begin{tabular}{lcccc}
\hline \multicolumn{1}{c}{ Model } & $\mathrm{B}$ & $\mathrm{SE}$ & $\mathrm{t}$ & $\mathrm{Sig}$ \\
\hline $\begin{array}{l}\text { Baseline (Organisation } \\
\text { does not adhere to PIG }\end{array}$ & -0.563 & 0.236 & -2.381 & 0.020 \\
$\begin{array}{l}\text { policy) } \\
\begin{array}{l}\text { Organisation adheres } \\
\text { to PIG }\end{array}\end{array}$ & 0.716 & 0.267 & 2.686 & 0.009 \\
\hline
\end{tabular}

Table 11 below shows the results of one simple linear regression analysis. This was calculated to predict EBPAS requirement scale based on the organisation adherence to NICE. A significant regression equations was found $(F(1,68)=$ 4.322, $p=0.041, R^{2}$ of 0.244$)$. These results indicate that those respondents whose organisations adhere to the NICE policy, on average, scoring significantly higher on the requirements scale than those who don't adhere who are scoring significantly lower in the baseline.

Table 11: Factor 1 of analysis 3, EBPAS scale Requirement with Organisation adheres to National Institute for Clinical Excellence (NICE)

\begin{tabular}{lcccc}
\hline \multicolumn{1}{c}{ Model } & $\mathrm{B}$ & $\mathrm{SE}$ & $\mathrm{t}$ & $\mathrm{Sig}$ \\
\hline $\begin{array}{l}\text { Baseline (Organisation } \\
\text { does not adhere to }\end{array}$ & -0.486 & .259 & -1.876 & 0.065 \\
$\begin{array}{l}\text { NICE) } \\
\begin{array}{l}\text { Organisation adheres } \\
\text { to NICE }\end{array}\end{array}$ & 0.597 & .287 & 2.079 & 0.041 \\
\hline
\end{tabular}

Table 12 below shows the results of one simple linear regression analysis. This was calculated to predict EBPAS requirement scale based on the organisation adherence to WTP. A significant regression equations was found $(F(1,68)=$ 4.150, $p=0.046 R^{2}$ of 0.240 ). These results indicate that those respondents, whose organisations adhere to the practice policy WPT, are on average scoring significantly higher on the requirements scale than those who don't adhere who are scoring significantly lower in the baseline. 
Table 12: Factor 1 of analysis 3, EBPAS scale Requirement with Organisation Adheres to Welsh Psychological Therapies (WPT)

\begin{tabular}{lcccc}
\hline \multicolumn{1}{c}{ Model } & B & SE & t & Sig \\
\hline $\begin{array}{l}\text { Baseline (Organisation } \\
\text { does not adhere to }\end{array}$ & -0.76 & 0.118 & -0.644 & 0.522 \\
$\begin{array}{l}\text { WPT) } \\
\begin{array}{l}\text { Organisation adheres } \\
\text { to WPT }\end{array}\end{array}$ & 0.759 & 0.372 & 2.037 & 0.046 \\
\hline
\end{tabular}

\subsubsection{Factor 2 of Analysis 3, EBPAS Openness with Primary Discipline 'Psychiatry'}

Table 13 below shows the results of one simple linear regression analysis. This was calculated to predict EBPAS openness scale based on the primary discipline variable psychiatry.

A significant regression equation was found $\left(F(3,66)=2.216, \mathrm{p}=0.023, \mathrm{R}^{2}=\right.$ 0.092). The results indicate that the psychiatrists were scoring lower on average than the nurses on the openness scale. The psychiatrists were less likely than the nurses to follow a treatment manual, to try therapy interventions developed by researchers, to use therapy/interventions, to try therapy/interventions different than usual. Differences between the psychologists and the therapists relative to the nurses on the openness scale are not statistically significant.

Table 13: Factor 2 of analysis 3, EBPAS openness scale with Primary discipline

\begin{tabular}{ccccc}
\hline Model & $\mathrm{B}$ & $\mathrm{SE}$ & $\mathrm{t}$ & $\mathrm{Sig}$ \\
\hline Baseline (nurses) & 0.142 & .155 & 0.915 & 0.363 \\
Primary discipline & -0.221 & .297 & -0.741 & 0.461 \\
recode psychology & & & & \\
$\begin{array}{l}\text { Primary discipline } \\
\text { recode psychiatry }\end{array}$ & -0.714 & .306 & -2.331 & 0.023 \\
$\begin{array}{l}\text { Primary discipline } \\
\text { recode therapsw }\end{array}$ & 0.152 & .328 & 0.463 & 0.645 \\
\hline
\end{tabular}




\subsection{EBPAS Attitudinal Scores with Demographic Variables}

Table 14 below shows the results of two simple linear regression analysis on the EBPAS attitudinal score. The first model includes the independent variable primary discipline which comprises four categories: nursing is the baseline category against the remaining three categories (psychology, psychiatry and therapy).

This model explains a significant amount of variation in EBPAS attitudinal scores at the $5 \%$ level $\left(F(3,66)=3.421, \mathrm{p}=0.006, \mathrm{R}^{2}=0.135\right)$. The results indicate that the psychologists had a significantly lower mean EBPAS attitudinal score than the nurses. There were no statistically significant differences in mean EBPAS score between the psychologists and the therapists relative to the nurses.

Table 14: EBPAS Attitudinal score with Primary Discipline

\begin{tabular}{lcccc}
\hline Model & $\mathrm{B}$ & $\mathrm{SE}$ & $\mathrm{t}$ & $\mathrm{Sig}$ \\
\hline Baseline (nurses) & 5.883 & 0.130 & 45.113 & $<0.0005$ \\
$\begin{array}{l}\text { Primary discipline } \\
\text { recode psychology }\end{array}$ & -0.715 & 0.251 & -2.852 & 0.006 \\
$\begin{array}{l}\text { Primary discipline } \\
\text { recode psychiatry }\end{array}$ & -0.334 & 0.258 & -1.294 & 0.200 \\
$\begin{array}{l}\text { Primary discipline } \\
\text { recode therapsw }\end{array}$ & 0.133 & 0.277 & 0.480 & 0.633 \\
\hline
\end{tabular}




\subsubsection{EBPAS Attitudinal score with Practice Policy Welsh Psychological Therapies (WPT)}

Table 15 below shows the result of one simple linear regression analysis on the EBPAS attitudinal score. The first model includes the independent variable practice policy. The policy variable comprised of four categories: Welsh psychological therapies, the mental health policy implementation guideline, NICE clinical guideline on psychosis / schizophrenia and the mental health Wales measure. There is a distinction between question (above) primary discipline and question nine adherence to policy guidelines that the latter was a multi response question in that respondents could have chosen multiple selection answers whereas question six was a singular response in that respondents made one selection.

This model explains a significant amount of variation in EBPAS attitudinal scores at the $10 \%$ level $\left(F(1,68)=3.413, \mathrm{p}=0.069, \mathrm{R}^{2}\right.$ of 0.048$)$. These results indicate that those respondents who do work in organisations which adhere to the Welsh psychological therapies (WPT) policy are more likely at the $10 \%$ level if not quite at the $5 \%$ level to adopt the policy than the baseline respondents (which is subsumed in the baseline). The baseline respondents in this particular model are those who work in organisations which do not adhere to the WPT policy.

Table 15: EBPAS Attitudinal score with Practice Policy Welsh Psychological Therapies (WPT)

\begin{tabular}{lcccc}
\hline \multicolumn{1}{c}{ Model } & $\mathrm{B}$ & $\mathrm{SE}$ & $\mathrm{t}$ & $\mathrm{Sig}$ \\
\hline $\begin{array}{l}\text { Baseline (Organisation } \\
\text { do not adhere to WPT) }\end{array}$ & 5.653 & 0.100 & 56.283 & $<0.0005$ \\
$\begin{array}{l}\text { Organisation adheres } \\
\text { to WPT }\end{array}$ & 0.587 & 0.318 & 1.847 & 0.069 \\
\hline
\end{tabular}




\subsection{Statistical modelling and interactions}

The statistical modelling section is comprised of the demographics and organisational variables. The demographics will be presented first and then the organisation variables along with the demographic variables.

\subsubsection{Demographics}

\section{Model 1: Age by Gender, requirement scale}

Table 16 below shows the result of one simple linear regression analysis. The first model includes the independent variable gender with the requirement factor as the dependent variable. There is no significant result with this model $(F(1$, $68)=0.488, p=0.487, R^{2}$ of 0.007$)$. These results indicate that the female respondents scored higher on average on the requirements scale than the male respondents, but not significantly so.

Table 16: Model 1 Gender main effects

\begin{tabular}{lcccc}
\hline \multicolumn{1}{c}{ Variable } & $\mathrm{B}$ & $\mathrm{SE}$ & $\mathrm{t}$ & $\mathrm{p}$ \\
\hline $\begin{array}{l}\text { Baseline } \\
\text { (males) }\end{array}$ & -0.085 & 0.167 & -0.508 & 0.613 \\
Females: males & 0.161 & 0.230 & 0.699 & 0.487 \\
\hline
\end{tabular}

\section{Model 2: Age main effects}

Table 17 below shows the result of one simple linear regression analysis. The second model includes the independent variable new binary age with the requirement factor as the dependent variable. This model explains a significant amount of variation at the $10 \%$ level $\left(F(1,68)=3.340, \mathrm{p}=0.047, \mathrm{R}^{2}\right.$ of 0.047$)$. The males and the females were scoring lower on the requirement scale than the younger male's respondents which are subsumed in the baseline.

\section{Table 17: Model 2 Age main effects}

\begin{tabular}{lcccc}
\hline \multicolumn{1}{c}{ Variable } & $\mathrm{B}$ & $\mathrm{SE}$ & $\mathrm{t}$ & $\mathrm{p}$ \\
\hline Baseline & 0.188 & 0.153 & 1.236 & 0.221 \\
younger males & & & & \\
Older: younger & -0.412 & 0.226 & -1.828 & 0.072 \\
\hline
\end{tabular}




\section{Model 3: Age and Gender main effects}

Table 18 below shows the results of linear regression analysis. This model includes the main effects of gender and age with the requirement factor as the dependent variable. This model explains $5 \%$ of the variation in the requirement factor which is approaching significance at the $10 \%$ level $(F(2,67)=1.783, p=$ $0.176, R^{2}$ of 0.051 ). The older respondents are scoring lower on average on the requirements scale than the younger respondents who are subsumed in the baseline category. The females score higher on average than the males.

\section{Table 18: Model 3 Gender and Age main effects}

\begin{tabular}{lcccc}
\hline \multicolumn{1}{c}{ Variable } & $\mathrm{B}$ & $\mathrm{SE}$ & $\mathrm{t}$ & $\mathrm{p}$ \\
\hline Baseline & 0.121 & 0.202 & 0.598 & 0.552 \\
younger males & & & & \\
Females: males & 0.117 & 0.228 & 0.512 & 0.610 \\
Older: younger & -0.399 & 0.228 & -1.750 & 0.085 \\
\hline
\end{tabular}

The ANOVA table 19 indicates that the interaction between age and gender is approaching significant at the $0.1 \%$ level. This means that the gender effect is varying significantly between age groups. Alternatively the age effect varies significantly between the males and the females.

The fourth model includes the independent variable age, gender plus the interaction between age and gender with the requirement factor as the dependent variable. This model explains a significant amount of variation at the $10 \%$ level $\left(F(3,66)=2.354, p=0.080, R^{2}\right.$ of 0.097$)$.

The estimates of the parameters for regression coefficients in model 4 are presented in the column headed ' $\mathrm{B}$ ' in table 20.

The older females are scoring lower on the requirement scale than the younger males who are subsumed into the baseline category. The younger females score higher on average than the younger males. These parameter estimates are used to calculate the predicted value of the requirements factor for all four combinations of gender and age group. These four predicted values are plotted on figure 13 . 
Model 4: Age and Gender plus interactions

Table 19 below shows the result of linear regression analysis.

Table 19.1: ANOVA table between age and gender

\begin{tabular}{lccccc}
\hline \multicolumn{1}{c}{ Model } & SS & DF & MS & F & Sig \\
\hline Model 3: & 3.187 & 2 & 1.594 & 1.85 & $\mathrm{p}>0.05$ \\
$\begin{array}{l}\text { Age and } \\
\text { gender } \\
\text { (Main Effects }\end{array}$ & & & Df 2,66 & 0.165 \\
only) & & & & \\
Model 4: & 2.91 & 1 & 2.91 & 3.37 & $\mathrm{p}>0.10$ \\
$\begin{array}{l}\text { Model 3 plus } \\
\text { Age by }\end{array}$ & & & & & \\
Gender & & & & & \\
interaction & & & & & \\
$\begin{array}{l}\text { Residual } \\
\text { Total }\end{array}$ & 56.971 & 66 & 0.863 & & \\
\hline
\end{tabular}

Table 19.2: Model 4 Gender and Age plus interactions

\begin{tabular}{lcccc}
\hline \multicolumn{1}{c}{ Variable } & $\mathrm{B}$ & $\mathrm{SE}$ & $\mathrm{t}$ & $\mathrm{p}$ \\
\hline Baseline & -0.100 & 0.232 & -0.429 & 0.669 \\
younger males & & & & \\
Older: younger & 0.029 & 0.324 & 0.089 & 0.929 \\
$\begin{array}{l}\text { Females: males } \\
\text { Age by gender }\end{array}$ & 0.498 & 0.305 & 1.631 & 0.108 \\
$\begin{array}{l}\text { interaction } \\
\text { (older females) }\end{array}$ & -0.824 & 0.449 & -1.836 & 0.071 \\
\hline
\end{tabular}


Figure 15: A line graph showing Age by Gender Interaction

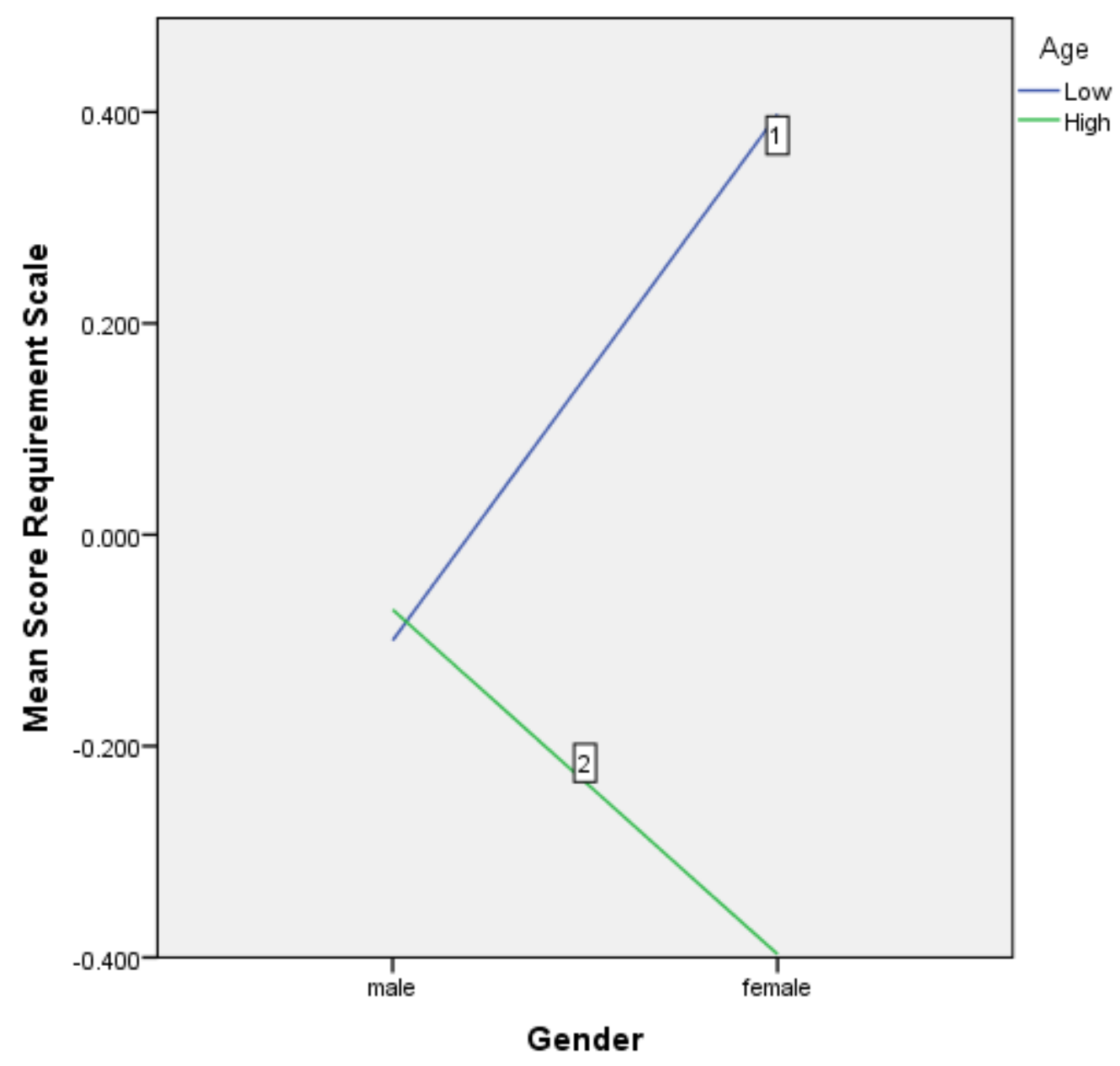

Interpretation of Figure 15

The line graph shows age by gender interaction. Line 1 signifies young age group and line 2 signifies older age group.

In the younger age group the females score higher on the EBPAS requirements scale than the males where as in the older age group the males are outperforming the females.

For the males' there is little to choose between the two age groups where as for the females' the mean on the requirement scale is markedly higher in the younger age group. 


\section{Models 1 to 4: Primary discipline and service model (Requirement scale)}

A series of models 1 to 4 was fitted with primary discipline and service model (inpatient, outpatient, case management, and day treatment) with the requirement scale. There were no significant effects within the models so these results are not presented here (see Appendix 14).

\subsection{Organisational Variables}

\section{Gender by Policy (Requirements scale)}

The policy variable, 'does your organisation adhere to the following practice policies' was a multi response categorical variable which provided the opportunity for respondents to choose all policies that applied to their organisations. It is worth noting that there are differing policy contexts in England and Wales and this is reflected in how the respondents stipulated adherence to these policies. The frequencies are presented in Table 22 below. Forty-five of the respondents from England stipulated that their organisation did adhere to the Policy Implementation Guidelines (PIG) compared to 10 respondents in Wales. For the National Institute for Clinical Excellence Policy (NICE), 48 of the respondents from England stipulated that their organisation adhered to this policy compared to 9 respondents in Wales. For the Welsh policies the Wales psychological therapies (WPT) 53 of the England respondents stipulated that they their organisation didn't adhere to compared to 10 of the Wales respondents. The Wales Mental Health Measure (WMHM) again 53 of the England respondents didn't adhere compared to 16 of the Welsh respondents.

Table 20: Frequencies on adherence to practice policies

\begin{tabular}{lccc}
\hline \multicolumn{1}{c}{ Policy } & England & Wales & Totals \\
\hline PIG: yes & 45 & 10 & 55 \\
PIG: no & 8 & 7 & 15 \\
\hline NICE: yes & 48 & 9 & 57 \\
NICE: no & 5 & 8 & 13 \\
\hline WPT: yes & 0 & 10 & 10 \\
WPT: no & 53 & 7 & 60 \\
\hline WMHM: yes & 0 & 16 & 16 \\
WMHM: no & 53 & 1 & 54 \\
\hline
\end{tabular}


The two zero counts in Table 20 are classified as structural zeros. These two cells with structural zeros have an expected value of zero. This means that no observations could fall into that cell, in other words, none of the English respondents could be expected to adhere to Welsh policies and measures.

Berger and Zhang (2005), "Structural zero are not part of the data, therefore they do not contribute to the likelihood function or model fitting. Contingency table containing structural zero is in same sense an incomplete table the A usual chi square test cannot be applied directly" (1958).

\subsubsection{Models of Gender and Policy Main Effects Plus Gender and Policy Interaction}

A series of models were computed involving gender and policy main effects, and the gender by policy interaction. The models included the following effects: gender main effects only; policy main effects only; gender and policy main effects; gender and policy main effects plus gender by policy interaction. WPT was significant as a main effect only. There were no significant results within the models (see Tables 12 to 16 for full model results in Appendix 14).

\section{Model 2: Policy main effects}

Table 21 below shows the result of linear regression analysis. The second model includes the independent variables practice policy implementation guideline (PIG), Wales mental health measure (WMHM) and the Welsh Psychological Therapies with the requirement factor as the dependent variable. This model explains a significant amount of variation $(F(4,65)=3.877, \mathrm{p}=$ $0.007, R^{2}$ of 0.193 ). Of those respondents that adhere to WPT score significantly lower than respondents whose organisation adheres to PIG and WMHM. 
Table 21: Model 2: Policy main effects

\begin{tabular}{|c|c|c|c|c|}
\hline Variable & $B$ & SE & $\mathrm{t}$ & $p$ \\
\hline Baseline (NICE) & -0.342 & 0.271 & -1.264 & 0.211 \\
\hline $\begin{array}{l}\text { Organisation } \\
\text { adheres to PIG }\end{array}$ & 0.453 & 0.290 & 1.561 & 0.123 \\
\hline $\begin{array}{l}\text { Organisation } \\
\text { adheres to } \\
\text { WMHM }\end{array}$ & 1.124 & 0.491 & 2.291 & 0.025 \\
\hline $\begin{array}{l}\text { Organisation } \\
\text { adheres to WPT }\end{array}$ & -0.552 & 0.353 & -1.562 & 0.123 \\
\hline
\end{tabular}

\section{Model 3: age and policy main effects}

Table 22 below shows the result of linear regression analysis. The third model includes the independent variables age, practice policy implementation guideline (PIG), Wales mental health measure (WMHM) and the Welsh Psychological Therapies with the requirement factor as the dependent variable. This model explains a significant amount of variation $(F(4,65)=3.877, \mathrm{p}=$ $0.007, R^{2}$ of 0.193$)$.

Table 22: Model 3: Age and policy main effects

\begin{tabular}{|c|c|c|c|c|}
\hline Variable & $\mathrm{B}$ & SE & $\mathrm{t}$ & $p$ \\
\hline Baseline (NICE) & -0.165 & 0.291 & -0.566 & 0.573 \\
\hline Age (younger) & -0.339 & 0.217 & -1.557 & 0.124 \\
\hline $\begin{array}{l}\text { Organisation } \\
\text { adheres to PIG }\end{array}$ & 0.406 & 0.289 & 1.404 & 0.165 \\
\hline $\begin{array}{l}\text { Organisation } \\
\text { adheres to } \\
\text { WMHM }\end{array}$ & -0.488 & 0.352 & -1.386 & 0.171 \\
\hline $\begin{array}{l}\text { Organisation } \\
\text { adheres to WPT }\end{array}$ & 1.123 & 0.485 & 2.314 & 0.024 \\
\hline
\end{tabular}

\section{Model 4: Age and policy main effects plus interaction}

Table 23 below shows the result of linear regression analysis. The fourth model includes the independent variables new binary age, practice policy implementation guideline (PIG), Wales mental health measure (WMHM) and the Welsh Psychological Therapies plus the interaction variables with the requirement factor as the dependent variable. This model explains a significant amount of variation $\left(F(7,62)=3.571, \mathrm{p}=0.003, \mathrm{R}^{2}\right.$ of 0.287$)$. 
Table 23: Model 4: Age and Policy main effects plus interactions

\begin{tabular}{|c|c|c|c|c|}
\hline Variable & $B$ & SE & $\mathrm{t}$ & $p$ \\
\hline $\begin{array}{l}\text { Baseline } \\
\text { (younger) }\end{array}$ & -0.791 & 0.358 & -2.208 & 0.031 \\
\hline Age (older) & 0.983 & 0.529 & 1.860 & 0.068 \\
\hline $\begin{array}{l}\text { Organisation } \\
\text { adheres to PIG }\end{array}$ & 1.162 & 0.385 & 3.017 & 0.004 \\
\hline $\begin{array}{l}\text { Organisation } \\
\text { adheres to } \\
\text { WMHM }\end{array}$ & -0.450 & 0.519 & -0.868 & 0.389 \\
\hline $\begin{array}{l}\text { Organisation } \\
\text { adheres to WPT }\end{array}$ & 0.910 & 0.707 & 1.287 & 0.203 \\
\hline $\begin{array}{l}\text { Age by PIG } \\
\text { interaction }\end{array}$ & -1.628 & 0.570 & -2.857 & 0.006 \\
\hline $\begin{array}{l}\text { Age by WMHM } \\
\text { interaction }\end{array}$ & -0.545 & 0.705 & -0.773 & 0.442 \\
\hline $\begin{array}{l}\text { Age by WPT } \\
\text { interaction }\end{array}$ & 0.932 & 0.961 & 0.970 & 0.336 \\
\hline
\end{tabular}

The two non significant interaction terms (age by WMHM and age by WPT) were removed from model $4 \mathrm{a}$. The two non significant main effects (organisation adheres to WPT and organisation adheres to WMHM) were taken out of the model in order to test for the significance of the one remaining interaction term (age by PIG), the reduced main effects model $3 b$ first before adding the age by PIG interaction (model $4 b$ ).

Table 24: ANOVA: age and policy main effects plus policy Interaction (PIG and Welsh and Mental health measure)

The ANOVA table below goes with the previous table of model results.

\begin{tabular}{lccccc}
\hline \multicolumn{1}{c}{ Model } & SS & DF & MS & F & Sig \\
\hline $\begin{array}{l}\text { Model 3a: } \\
\begin{array}{l}\text { Age and } \\
\text { policy (Main }\end{array}\end{array}$ & 12.149 & 4 & 3.037 & 4.189 & 0.005 \\
$\begin{array}{l}\text { Effects only) } \\
\text { Model 4a: }\end{array}$ & & & & & \\
$\begin{array}{l}\text { Model 3a } \\
\text { plus Age by }\end{array}$ & 5.973 & 3 & 1.991 & 2.746 & 0.050 \\
$\begin{array}{l}\text { policy } \\
\text { interactions }\end{array}$ & & & & Df 3, 62 & \\
$\begin{array}{l}\text { Residual } \\
\text { Total }\end{array}$ & 44.946 & 62 & 0.725 & & \\
\hline
\end{tabular}


Table 25 shows an ANOVA age and PIG main effects plus age by PIG interactions. There was a significant main effect $(p=0.002)$ at the $1 \%$ level.

Table 25: ANOVA: age and PIG main effects plus age by PIG interactions

\begin{tabular}{lccccc}
\multicolumn{1}{c}{ Model } & SS & DF & MS & F & Sig \\
\hline Model 4b: & 14.812 & 4 & 3.703 & 1.269 & 0.292 \\
$\begin{array}{l}\text { PIG (Main } \\
\text { Effects only) }\end{array}$ & & & & Df 4,62 & \\
$\begin{array}{l}\text { Model 4b: } \\
\begin{array}{l}\text { Age by PIG } \\
\text { interactions }\end{array}\end{array}$ & 2.361 & 3 & 0.787 & $\begin{array}{c}0.269 \\
\text { Df 3,62 }\end{array}$ & 0.048 \\
$\begin{array}{l}\text { Residual } \\
\text { Total }\end{array}$ & 51.957 & 62 & 2.916 & & \\
\hline
\end{tabular}

Table 26 shows the main effect plus interactions. There is a significant age by PIG interaction $(p=0.048)$ at the $5 \%$ level.

Table 26: ANOVA Age by policy (1 category PIG)

\begin{tabular}{lccccc}
\hline \multicolumn{1}{c}{ Model } & SS & DF & MS & F & Sig \\
\hline $\begin{array}{l}\text { Model 3b: } \\
\begin{array}{l}\text { Age and } \\
\text { policy (Main }\end{array}\end{array}$ & 7.914 & 2 & 3.957 & 5.028 & 0.009 \\
$\begin{array}{l}\text { Effects only) } \\
\text { Model 4b: }\end{array}$ & 3.196 & 1 & 3.196 & $\begin{array}{c}4.061 \\
\text { Df 2, 66 }\end{array}$ & 0.048 \\
$\begin{array}{l}\text { Model 3b } \\
\text { plus Age by }\end{array}$ & & & & & \\
$\begin{array}{l}\text { policy } \\
\text { interaction }\end{array}$ & & & & & \\
$\begin{array}{l}\text { Residual } \\
\text { Total }\end{array}$ & 51.957 & 66 & 0.787 & & \\
\hline
\end{tabular}


Figure 16: Age by Policy interaction

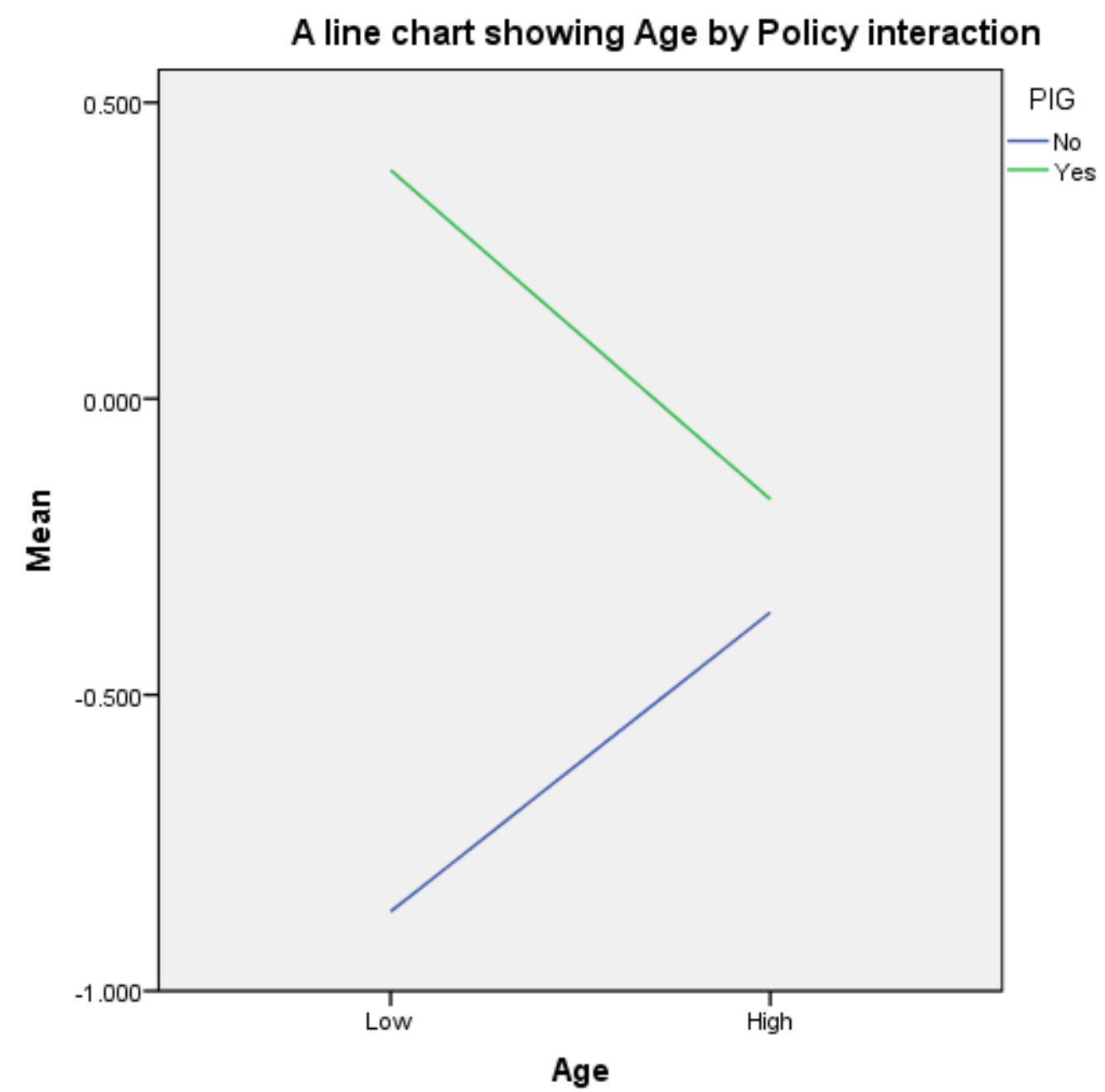

Interpretation of the line chart

In Figure 16 the younger respondents that do not adhere to the PIG score lower than those younger respondents who do adhere to the PIG.

The older respondents that do not adhere to PIG score higher than the older respondents who do adhere to PIG.

The difference between 'PIG-yes' and 'PIG-no' is markedly higher in lower age group. Or alternative interpretation: 'PIG-no': positive association between age and mean on requirements scale 'PIG-yes': negative association between age and mean scale. 


\subsection{Finding the Most Parsimonious Model of the Requirements Scale: Defining the Full Model}

\section{Model 6: primary discipline by inpatient plus interactions}

Table 27 below shows the result of linear regression analysis. The sixth model includes the independent main effect variables of the following: primary discipline recodes psychology, psychiatry and therapy / social work and type of service model provided (inpatient) plus the interaction variables with the requirement factor as the dependent variable. There is a significant result at the $10 \%$ level within this model $\left(F(7,62)=1.210, p=0.311, R^{2}\right.$ of 0.120$)$. The psychiatrists and psychologists are scoring lower than the nurses (subsumed in the baseline) on the requirements scales.

Table 27: Model 6 primary discipline by service model (inpatient) main effects plus interactions

\begin{tabular}{|c|c|c|c|c|}
\hline Variable & $\mathrm{B}$ & SE & $\mathrm{t}$ & $p$ \\
\hline Baseline (nurses) & 0.012 & 0.176 & 0.069 & 0.946 \\
\hline Primary discipline psychology & -0.052 & 0.335 & -0.156 & 0.877 \\
\hline Primary discipline psychiatry & -0.706 & 0.378 & -1.868 & 0.067 \\
\hline Primary discipline therapsw & 0.483 & 0.361 & 1.337 & 0.186 \\
\hline $\begin{array}{l}\text { Types of services provided by } \\
\text { your service model inpatient }\end{array}$ & 0.253 & 0.424 & 0.596 & 0.553 \\
\hline $\begin{array}{l}\text { Psychology by inpatient } \\
\text { interaction }\end{array}$ & -0.878 & 0.842 & -1.043 & 0.301 \\
\hline $\begin{array}{l}\text { Psychiatry by inpatient } \\
\text { interaction }\end{array}$ & 0.665 & 0.718 & 0.926 & 0.358 \\
\hline $\begin{array}{l}\text { Therapies by inpatient } \\
\text { interaction }\end{array}$ & -0.718 & 1.084 & -0.663 & 0.510 \\
\hline
\end{tabular}

\section{Model 6: primary discipline by outpatient plus interactions}

Table 28 shows the result of a linear regression analysis. The seventh model includes the independent main effect variables of the following: primary discipline re-codes psychology, psychiatry and therapy / social work. Also type of service model provided (outpatient), with interaction variables, the requirement factor as the dependent variable. There are significant results at 
the $5 \%$ and $10 \%$ level within this model $\left(F(7,62)=1.536, p=0.172, R^{2}\right.$ of 0.148).

Notably the psychiatrists are scoring lower than the other respondents in the base line category subsumed in the constant. The therapists and social workers are scoring higher than the other respondents who are subsumed in the baseline (nurses).

Table 28: Model 6 primary discipline by service model (outpatient) main effects plus interactions

\begin{tabular}{lcccc}
\hline \multicolumn{1}{c}{ Variable } & $\mathrm{B}$ & $\mathrm{SE}$ & $\mathrm{t}$ & $\mathrm{p}$ \\
\hline Baseline (nurses) & 0.032 & 0.176 & 0.181 & 0.857 \\
Primary discipline psychology & -0.310 & 0.357 & -0.868 & 0.389 \\
$\begin{array}{l}\text { Primary discipline psychiatry } \\
\text { Primary discipline therapsw }\end{array}$ & -1.021 & 0.498 & -2.052 & 0.044 \\
$\begin{array}{l}\text { Types of services provided by } \\
\text { your service model outpatient }\end{array}$ & 0.657 & 0.373 & 1.761 & 0.083 \\
$\begin{array}{l}\text { Psychology by outpatient } \\
\text { interaction }\end{array}$ & 0.118 & 0.393 & 0.299 & 0.766 \\
$\begin{array}{l}\text { Psychiatry by outpatient } \\
\text { interaction }\end{array}$ & 0.343 & 0.684 & 0.501 & 0.618 \\
$\begin{array}{l}\text { Therapies by outpatient } \\
\text { interaction }\end{array}$ & 0.786 & 0.693 & 1.134 & 0.261 \\
\hline
\end{tabular}

The interaction between primary discipline, and day treatment was not significant (See Appendix 14 for model results)

Table 29 below shows the result of the final model for the regression analysis. This model includes all significant main effects (age, PIG), significant interactions (age by gender; and age by PIG), and non-significant main effects (qualification, service model, years of experience and primary discipline) with the requirement factor as the dependent variable. This model is significant at the $5 \%$ level $\left(F(17,52)=2.159, \mathrm{p}=0.017 \mathrm{R}^{2}\right.$ of 0.414$)$. Deduction was done manually, until the most parsimonious model remained. This is an application of the principle of parsimony or Ockham's razor (Sober, 1981) presented. The model presents significant finding at below $5 \%$ level $(F(5,64)=6.77$, p < $0.0005, R^{2}$ of 0.364$)$. Also, the model demonstrates a significant amount of variation in the factor requirement, $p<0.0005$. Those respondents who are 
Dr/Fellow are more likely to score significantly more highly on the requirements scale than those respondents who not $\mathrm{Dr}$ or Fellow.

Table 29: The most parsimonious model of the requirements scale

\begin{tabular}{|c|c|c|c|c|}
\hline Variable & $\mathrm{B}$ & SE & $\mathrm{t}$ & $\mathrm{p}$ \\
\hline Baseline & -0.201 & 0.260 & -0.773 & 0.442 \\
\hline Age & -0.536 & 0.201 & -2.661 & 0.010 \\
\hline $\begin{array}{l}\text { Educational level Dr or Fellow } \\
\text { (yes/no) }\end{array}$ & 1.295 & 0.299 & 0.585 & $<0.0005$ \\
\hline $\begin{array}{l}\text { Does your organisation } \\
\text { adhere to the following } \\
\text { practice policies PIG DG } \\
2001\end{array}$ & 0.638 & 0.244 & 2.619 & 0.011 \\
\hline Primary discipline psychology & -1.083 & 0.317 & -3.421 & 0.001 \\
\hline Primary discipline psychiatry & -0.983 & 0.300 & -3.281 & 0.002 \\
\hline
\end{tabular}

\subsubsection{Goodness of Fit}

Goodness of Fit of a linear regression model attempts to get at the issue of how well a model fits a given set of data, or how well it will predict a future set of observations. The famous Bayesian statistician George Box, who said, 'all models are wrong, but some are useful' (Box, 1987, p.424).

Often researchers choose significance level equal to $0.01,0.05$ or 0.10 ; but any value between 0 and 1 can be used. To test this method the chi-squared goodness of fit test to determine whether observed sample frequencies differ significantly from those frequencies predicted by the final model. Table 30 below shows the comparison of observed values and EBPAS for the requirement scale. Assumptions checked by inspecting the histograms given of normality for EBPAS score total, requirements scale and openness scale are in Appendix 3. 
Table 30: Comparison of observed and predicted values for EBPAS requirement scale

\begin{tabular}{lrrrrr}
\hline & \multicolumn{1}{c}{ Minimum } & Maximum & \multicolumn{1}{c}{ Mean } & \multicolumn{1}{c}{ SD } & N \\
\hline Predicted & -1.6196890 & 1.6478810 & 0.0000000 & .57718689 & 70 \\
value & -1.79552543 & 1.56954312 & 0.00000000 & .76215543 & 70 \\
Residual & -2.806 & 2.855 & 0.000 & 1.000 & 70 \\
Std. Predicted & -2.251 & 1.968 & 0.000 & 0.956 & 70 \\
value & & &
\end{tabular}

Figure 17: Goodness of Fit for EBPAS Requirements

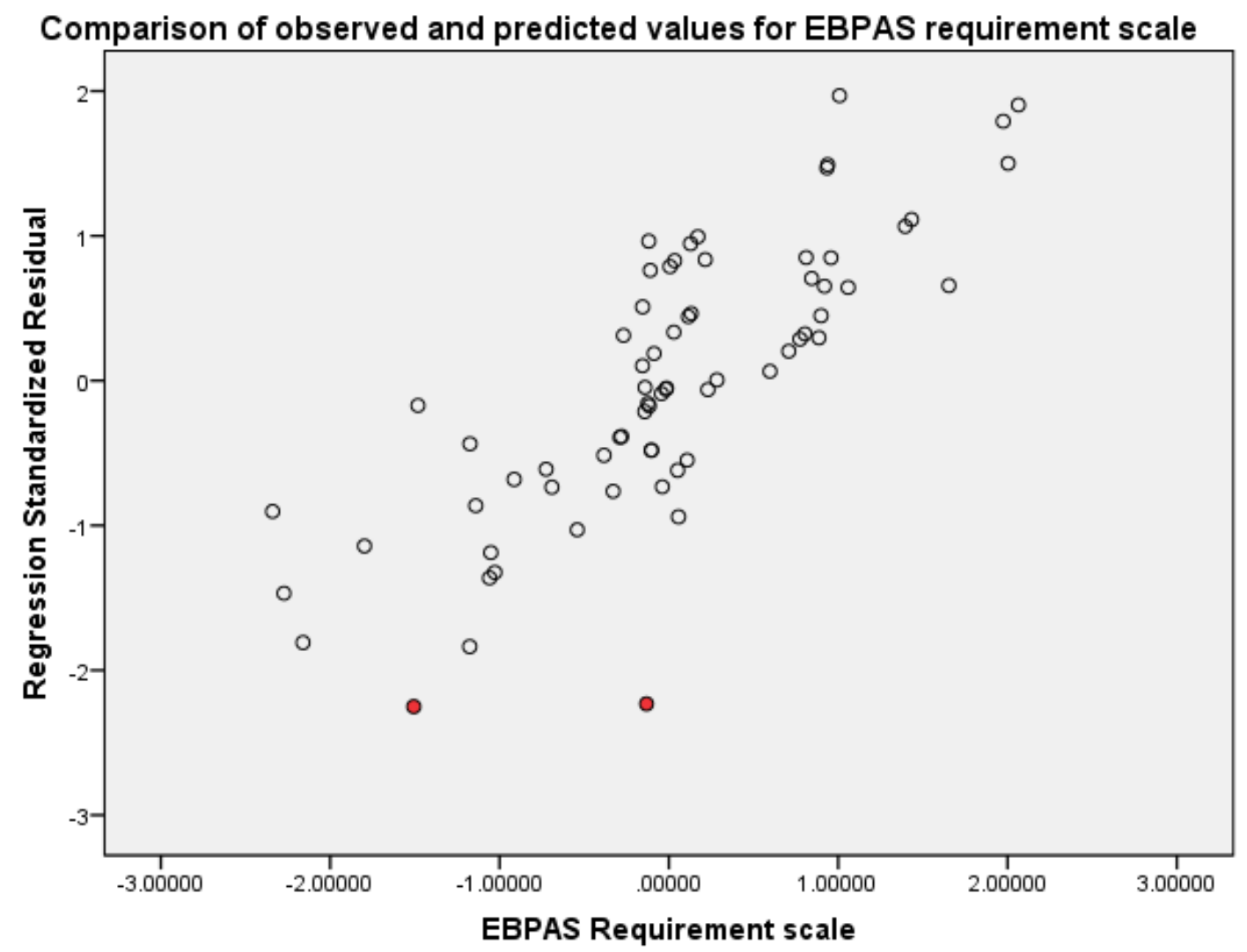


Figure 17 shows the goodness of fit for the requirements model. This model is doing well at factor score 1, for most respondents with a factor score close to zero, centred on zero. For those respondents with a high observed factor score; the model is under predicting, (predicted factor scores lower than the observed factor scores). For those observed with a low factor score the model is over predicting (predicted factor scores higher than the observed factor scores). The goodness of Fit shows outliers for row 54 and row 55 (red). The model is performing poorly for these cases. Upon examination the participants' characteristics these respondents had certain combination of characteristics which were interesting. The characteristics of the two respondents were as follows: outlier one stipulated that they had a professional qualification with a fellowship qualification within a hub and spoke model in an urban area. The second outlier had a professional qualification, degree and $\mathrm{PhD}$ within the discipline of psychology who also stated that the main service provided was a hub and spoke model within an urban area. Usually, hub and spoke models are provided in rural areas only. The National Collaborating Centre for Mental Health (2016) says that if the hub and spoke model is used improperly (for example in urban or suburban areas) as a means of reducing short-term costs, this can have negative impacts on clinical effectiveness, service user and carer and carer experience.

\subsubsection{The most Parsimonious Model: Final Model for EBPAS Total Score}

Table 33 below shows the result of the most parsimonious model for the regression analysis. This model includes all independent significant main effects (age, gender, PIG), significant interactions (age by gender; and age by PIG), and non significant main effects (qualification, service model, years of experience and primary discipline) with the EBPAS factor as the dependent variable.

There are no significant results within this model $(F(2,67)=5.051, \mathrm{p}=0.009$ $\mathrm{R}^{2}$ of 0.131 ). On average, those respondents whose organisations adhere to WPT score significantly higher at the $1 \%$ level on the total EBPAS score than those whose organisations do not. 
On average, the older respondents' score lower on the total EBPAS score than younger respondents, and, this difference is approaching significance at the $5 \%$ level.

Table 31: The most parsimonious model of the EBPAS scale

\begin{tabular}{|c|c|c|c|c|}
\hline Variable & $B$ & SE & $t$ & $p$ \\
\hline Baseline & 2.777 & 0.071 & 39.113 & 0.000 \\
\hline Age & -0.185 & 0.103 & -1.797 & 0.077 \\
\hline $\begin{array}{l}\text { Does your organisation } \\
\text { adhere to the following } \\
\text { practice policies WPT }\end{array}$ & 0.472 & 0.172 & 2.751 & 0.008 \\
\hline
\end{tabular}

\subsection{Summary of Regression Analysis and Interactions}

\section{Requirements with age}

The older respondents were less likely to adopt a therapy or intervention if they received training if it was required by their supervisor, organisation and country than the younger respondents.

\section{Requirements with Policy Implementation Guideline (PIG)}

Respondents whose organisations adhere to the PIG policy, on average are scoring significantly higher on the requirements scale than those who don't adhere who are scoring significantly lower in the baseline.

\section{Requirements with National Institute for Health and Care Excellence (NICE)}

The respondents whose organisations adhere to the NICE policy, on average, scoring significantly higher on the requirements scale than those who don't adhere.

\section{Requirements with Welsh Psychological Therapies (WPT)}

The respondents, whose organisations adhere to the practice policy WPT, are on average scoring significantly higher on the requirements scale than those who don't adhere. 


\section{Openness with primary discipline}

The psychiatrists were less likely than the nurses to follow a treatment manual, to try therapy interventions developed by researchers, to use therapy/interventions, to try therapy/interventions different than usual.

\section{EBPAS score with primary discipline}

The results indicate that the psychologists had a significantly lower mean EBPAS attitudinal score than the nurses.

\section{EBPAS Welsh Psychological Therapies (WPT)}

The respondents who do work in organisations which adhere to the Welsh psychological therapies (WPT) policy are more likely at the $10 \%$ level if not quite at the $5 \%$ level to adopt the policy than the baseline respondents.

\section{Interactions}

Two interactions were found. (1) Age by gender and (2) age by policy interaction.

Age by gender interaction. In the younger age group the females scored higher on the EBPAS requirements scale than the males where as in the older age group the males are outperforming the females.

Age by policy interaction. The younger respondents that did not adhere to the PIG scored lower than those younger respondents who do adhere to the PIG. The older respondents that do not adhere to PIG scored higher than the older respondents who do adhere to PIG.

\subsection{Summary of Findings}

The primary finding of the quantitative component of this study is that attitudes towards the adoption of evidence based practices can be identified and assessed in Early Intervention Psychosis (EIP) practitioners in England and Wales. The EBPAS subscales represent four distinct constructs involving willingness to adopt and evidence based practice given their intuitive appeal, willingness to adopt new practices if required, general openness toward new or innovative practices if required, and openness toward new or innovative 
practices and perceived divergence of usual practice with academically developed or research based practices (Aarons, 2004).

My findings show that requirements and openness scales were good predictors of attitudes towards adopting evidence based practice (EBP).

Requirements with age varied significantly between older and younger respondents. The older respondents were less likely to adopt a therapy or intervention if they had received training. This might suggest that the older respondents may be placing preferential weight on their own clinical experience (Stewart \& Chambless, 2007).

The respondents whose organisations adhered to policies such as the Policy Implementation Guideline (PIG), National Institute for Health and Care Excellence (NICE) and the Welsh Psychological Therapies (WPT) on average scored higher on the requirements scale than those respondents who did not adhere to the policies. This suggests that those respondents scoring higher, work in more positive cultures than those working in organisations scoring significantly lower. With respondents scoring higher on the requirements scale indicating a more positive attitude toward adopting an evidence based practice if required to do so may highlight less bureaucratic organisations.

Openness varied with primary discipline. The psychiatrists were less likely than the nurses to follow a treatment manual, to try therapy interventions developed by researchers. Openness could be changed or developed in relation to exposure of training courses or delivery of interventions for psychosis (Aarons, 2004). Openness to innovation can be an important component of mental health organisational context in that it denotes in the development of learning organisations (Anderson \& West, 1998; Birleson, 1999 \& Garvin, 1993).

Psychologists had significantly lower EBPAS mean scores than the nurses. This could be a plethora of issues. It may be that there are other factors interacting with provider discipline in multifaceted ways. Barrick and Mount (1991) argue that providers with different personality characteristics may respond to organisational constraints in complex ways and such characteristics are important to consider in understanding and improving job performance. 
In chapter 5, I present my analysis of the qualitative findings of two themes generated from analysis of data collected in research interviews. These are sustaining evidence based practices and challenges and opportunities. I will show how practitioners talk about their experiences of delivering evidence based early intervention in psychosis services. 


\section{CHAPTER 5: Qualitative Findings from the Early Intervention Practitioner's Semi-Structured Interviews}

\subsection{Introduction}

In this chapter I present two themes arising from my analysis of research interviews with early intervention practitioners for this study. I will argue that the two themes identified are relevant factors in sustaining early intervention teams. These themes are sustaining evidence based practices and challenges and opportunities. The themes align with my research questions as follows; Theme one 'sustaining evidence based practices' addresses research question three, 'what do early intervention lead practitioners say are the crucial factors in sustaining or not sustaining practices in their teams in England and Wales?'. Theme two 'challenges and opportunities addresses research question two, 'to what extent is service context and culture relevant to practitioner's experiences of adopting evidence based practice?' and research question four, 'what do early intervention lead practitioners say are the facilitators and barriers to implementation of evidence based practices in their services?'.

My analysis shows the challenges and opportunities that arise in regard to the implementation of evidence-based practices within specialist mental health services. In the following sections I will show how the theme of 'sustaining early intervention practices' is constructed of multiple categories that focus on claims of the special nature of this work. Data collected and analysed for the current study suggests that there is a need to champion and assert the value of this work within services and there is a focus on dealing with contested notions of what constitutes evidence based practice in early intervention services. In theme two I examine the challenges and opportunities to adopting evidence based practices based on the accounts provided by early intervention practitioners. These focus on claims of barriers and protection of early intervention services to achieve outcomes which I argue is the justification provided for resources at an organisational level. 


\subsection{Selection of Interview Participants}

Of the seventy participants who completed the survey thirteen respondents stated that they did not wish to take part in the semi-structured interview. Fiftyseven participants stated that they would like to participate in the interview if contacted. I aimed to ensure that I had at least one participant in each of the sampling cells, e.g. high and low EBPAS scores, geographical area (urban, rural, suburban) and team establishment. I also selected nine participants in England and nine in Wales by design (see Table 32). Sampling stopped at eighteen interviews because of limitations on time and resources.

\subsection{Analysis of Data}

Qualitative research tends to use smaller samples than quantitative research (Braun \& Clarke, 2013). I did not aim to achieve data saturation. I aimed to collect data to provide a complete and truthful picture of the phenomenon at study. Guest et al., (2006) conducted experiments with data they had collected from in-depth interviews with women in two West African countries. They had conducted and collected and transcribed sixty interviews. They found that data saturation was achieved once around twelve transcripts had been thematically analysed suggesting it might be reasonable to assume that this number is sufficient to ensure that adequate coverage can be gained in qualitative research interviewing with small samples.

It became clear to me whilst reading through the transcripts horizontally line by line that participants were questioning the researcher on the questions being asked. For example, "Things like CBT, CBTP, family interventions, that sort of thing. Is that okay?" Another example from a participant's transcript, "were you just asking about barriers"? Another example, "I don't think I'm giving you the answers that you need really am I"? These questions where the participant is asking the researcher are illustrated by Mishler (1991) in his text on the joint meaning of construction. Mishler says that one-way an interview develops in through mutual reformulation and specification of questions, by which they take on particular and context bound shades of meaning. Mishler (1991) tells us that understanding the meaning of the respondent's "answer" depends on our 
recognition of it as an answer to our own specification of the question rather than to the original question asked by the interviewer. He proposes that questions asked have many possible meanings through which its intention may be realised, and in the exchange it has taken on only one meaning, the one specified by the respondent and accepted in turn by the interviewer. He tells us that rather than serving as a stimulus having a predetermined, presumably shared meaning and intended to elicit a response, a question may more usefully be thought of as part of a circular process through which its meaning and that of its answer is created in the discourse between interviewer and respondents as they try to make continuing sense of what they are saying to each other. 
Table 32: Selection of Interview Participants

\begin{tabular}{|c|l|l|c|c|c|}
\hline $\begin{array}{c}\text { Participant } \\
\text { No }\end{array}$ & Eng/Wales & \multicolumn{1}{|c|}{ Gender } & Job & $\begin{array}{c}\text { EBPAS } \\
\text { Scores }\end{array}$ & Urbal \\
\hline 1 & England & Female & Psychiatrist & 3.47 & Urbar \\
\hline 2 & England & Male & Manager & 2.53 & Urbar \\
\hline 3 & England & Female & Psychiatrist & 1.80 & Subu \\
\hline 4 & England & Female & Manager & 2.67 & Subu \\
\hline 5 & England & Male & Psychologist & 2.60 & Rural \\
\hline 6 & England & Male & Psychiatrist & 2.20 & Rural \\
\hline 7 & England & Female & Psychiatrist & 2.33 & Urbar \\
\hline 8 & England & Male & Psychologist & 1.93 & Rural \\
\hline 9 & England & Male & Nurse & 3.00 & Subu \\
\hline 10 & Wales & Male & Nurse & 2.87 & Urbar \\
\hline 11 & Wales & Female & Therapist & 3.73 & Rural \\
\hline 12 & Wales & Female & Recovery practitioner & 3.40 & Urbar \\
\hline 13 & Wales & Male & Nurse & 3.27 & Urbar \\
\hline 14 & Wales & Male & Nurse & 2.33 & Urbar \\
\hline 15 & Wales & Male & Psychologist & 2.80 & Urbar \\
\hline & & & Associate specialist & & \\
16 & Wales & Male & psychiatry & 1.73 & Subur \\
\hline 17 & Wales & Male & Psychiatrist & 2.60 & Rural \\
\hline 18 & Wales & Male & Nurse & 3.07 & Urbar \\
\hline
\end{tabular}




\subsection{Sustaining Evidence Based Practices}

This theme addresses research question three which is, 'what do early intervention lead practitioners say are the crucial factors in sustaining or not sustaining practices in their teams in England and Wales?'

In this theme I have collated claims made by participants who have constructed what can be read as important factors in sustaining early intervention teams within their organisations. The theme comprises three categories which are displayed in the visual representation below (Figure 18). The categories are: (1) what constitutes EBPs in relation to early intervention, (2) specialised practice and (3) champions. It has been previously argued that an important step in ensuring the widespread availability of evidence-based practices is to identify factors that promote both, the initial implementation of EBPs and their long term continuation over time (Bond, Drake \& McHugo, 2012; Shediac-Rizkallah \& Bone 1998), indicating sustainability in regard to the continuation of programs.

\section{Figure 18}

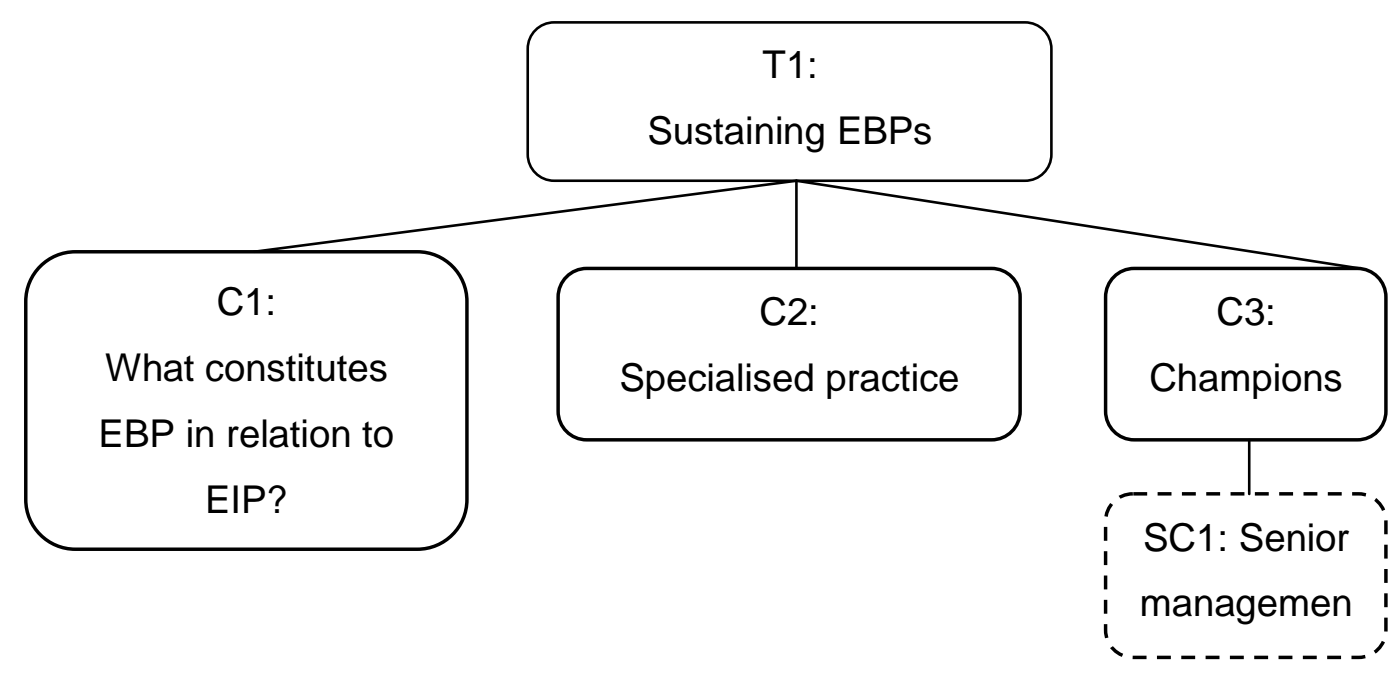

Figure 18: A visual representation of theme and categories

$\mathrm{T}=$ Theme; $\mathrm{C}=$ Category; $\mathrm{SC}=$ Sub category 


\subsection{Category 1: What Constitutes Evidence Based Practice in Relation to Early Intervention in Psychosis?}

\section{'What constitutes as evidence?': there is no simple answer!}

Participants were asked what they thought to be primary factors that would help maintain early intervention teams. My aim in doing so was to establish if there was an answer to the question, "Is there a common thread described by practitioners that reinforces the need for early intervention for early intervention teams'?

In the data extract below taken from a research interview with participant Richard, he advances a claim in relation to the robust effectiveness of evidence that EIP has delivered. He describes how the evidence for early intervention has filtered down to policy and how this has acted as a driver to sustaining EIP teams. Richard implies a direct link between evidence and policy and how this fits or does not fit with what we know about evidence based policy making.

"I think secondly, fortunately the robust evidence base for early intervention (meaning EIP) and that has finally kind of filtered down into the politics and Department of Health; so early intervention is a by-word now used within various political manifestos. Not necessarily that they completely understand what it means and what the evidence base is but, at least there does appear to be an understanding there". (Richard)

Richard positions early intervention in psychosis services as a kind of higher standard for evidenced based health delivery. He claims that, its meaning is not fully understood but there is an embedded understanding of the principle. This can be read as a form of special pleading, but it could also be interpreted as slightly naïve to think that evidence is prioritised over other contingencies in policy making.

The data extract below is taken from a research interview with Phil who is a consultant psychologist working in England at an improving access to psychological therapies for severe mental illness demonstration site. Phil's team has been established for six years, the main model used in his service being standalone specialist with case management being the main type of 
service provided. Phil has sixteen years' experience of working in mental health services. Phil describes how evidence based practice is not such a straightforward notion.

"I think Evidence Based Practice isn't such a straight forward notion so it's not a simple or uncontested domain as to what constitutes adequate evidence. So the question you ask opens up broader philosophical consideration of what constitutes evidence. There aren't many practitioners who would say um what I am delivering is not evidence based, but there are many practitioners who would say I am not delivering manualised CBT as recommend by NICE, so NICE guidelines don't have a monopoly on what constitutes Evidence Based Practice, I guess that's what I am saying". (Phil)

Phil talks about 'what constitutes evidence' and evidence that comes from NICE is not always implemented or delivered as per se. This returns to the question of how evidence is constituted and how we derive knowledge. Knowledge is obtained from clinician experience, patients and carers (Nutley, Powell \& Davies, 2013). Phil argues that EBP is not so clear cut as maybe initially thought of. Phil also disputes the primacy of guidelines and in part reflects awareness among knowledgeable practitioners that the methods used by NICE may not always capture all the relevant evidence and may prioritise certain types of knowledge over others (Guy, Thomas, Stephenson \& Loewenthal, 2011). Practitioners with many years of experience have learned to adapt their approach to interventions to better suit individuals they are treating. While this is likely to challenge ideas of fidelity it is nevertheless located in the real world of service delivery and non-research selected clinical populations where there is likely to be much more variation (Cleary, Hunt, Walter \& Jackson, 2010).

The data extract I present below is taken from a research interview with Steve who is a nurse by professional background. Steve advances a claim in relation to the notion of what constitutes Evidence Based Practice (EBP). Steve describes, 'what is good evidence, and what is not in terms of interventions that are being used' and how this is communicated (with what is coming through from NICE). 
"Then more broadly in terms of evidence-based practice I think also one of the difficulties is the general access of the work on the ground to an ongoing discussion in the area about what is evidence-based practice, what is good evidence and what is not in terms of the interventions that they are using. Stuff like NICE guidelines doesn't necessarily filter through to the teams in a very systematic way of making sure that all of the CMHT for instance are regularly keeping up to date with what is coming through from NICE to make sure that what they are doing is evidence-based practice”. (Steve)

Steve seems to indicate that practitioners may be somewhat be passive in expecting someone to 'filter through' information about what is 'up to date'. Of course it is understood that all professionals have a duty to keep up to date with the latest changes within their organisation which is a feature of 'codes of practice' for most professional groups (NMC, 2015 \& BPS, 2009). I acknowledge that there is a wider issue in terms of drift from a particular model. In using the term 'drift' I refer to the variation from the model and possibly increasing distance of time from initial training. There can be a drift from consensus on how best to deliver interventions (Mazzucchelli \& Sanders, 2010). I will pick up and return to this point again in theme two within the fidelity category.

Steve is not explicitly discussing fidelity, but he is indicating that in order to maintain fidelity and to work in evidence based ways requires teams to be aware of developments and have access to information within their field. NICE guidelines are freely available so there is a choice here for practitioners to either keep up to date or not and there should be no need for these guidelines to 'filter down'. An option for services is to address any passivity among staff by building in refresher courses and team supervision. Steve seems to be highlighting a need for something like this in his opening lines when he indicates that there is difficulty in frontline workers getting access 'to an ongoing discussion'.

One way of understanding this discussion is to view it as one between researchers, practitioners and policy makers. When looking at the 'what works' question different designs are placed in a hierarchy to decide the standard of evidence in support of a particular practice or programme (Nutely, Powell \& Davies, 2003). Some researchers may attach high levels of importance to the 
results of clinical trials and tend to view that clinical outcomes will be best. Practitioners are said to develop routines based on their procedural knowledge (Cohen, 1996). Policies are developed to create changes and improvements in services such as to improve the health of populations and change finding or accountability structures (Kane, 2002). Policy making is not conducted at one level, it occurs at national, regional and local levels. Groups involved in policymaking process include: government ministers, regional levels or government and regional officers of central government, local authorities, health authorities, NHS trusts and primary care groups and trusts, local practitioners and managers. It is not always the same evidence that is dominant at these levels. The question of what evidence is powerful, why, when, at what level is critical to establishing an evidence based policy framework for mental health.

Evidence from randomised control trials are important in everyday clinical practice. Guy et al., (2011) discuss doubtful consequences for patients of their inability to access the full range of psychotherapies due to a mixture of NICE's approach to mental health, and implementation of its guidance through to improving access to psychological therapies. For example while service user views were well represented in the NICE guideline on depression, when it came to making treatment recommendations their preferences were disregarded in favour of RCT evidence. This is somewhat evident in Phil's extract when he refers to NICE guidelines and that they don't have the monopoly of what constitutes evidence based practice, but Phil does assert a new claim that NICE may not capture all relevant evidence and may prioritise certain types of knowledge over others. Konnerup and Kongsted (2012) found that the majority of Cochrane Collaboration reviews are limited to considering RCTs only. Critics argue that this means that important evidence is overlooked and this weakens the value of evidence syntheses (Ogilvie, Egan, Hamilton \& Petticrew, 2005). Randomised control trials in psychiatry have had problems with flawed designs, small sample sizes, differences between settings and comparison groups, high attrition rates, fidelity of interventions and different outcome measures (Cleary, Hunt, Matheson, Siegfried \& Walter, 2010). 
There is a very substantial and growing literature reporting on the effectiveness of psychosocial interventions for people with severe mental illness (Lutgens, Gariepy \& Malla, 2017). The challenge both for practitioners and for service managers is to determine when evidence of effectiveness is sufficient for an intervention to be considered as being 'evidence based' or 'empirically supported' for application in mental health service. Evidence is considered to be knowledge derived from a range of sources. Titchen and Higgs (2000) describe knowledge as fundamental to reasoning and decision making and thus central to professional practice. Eraut $(1985,2000)$ has broadly categorised knowledge into two types; propositional (derived through research and scholarship) or codified knowledge and non-propositional (informal, implicit and derived through practice) or personal knowledge (life experiences and cognitive resources).

My findings from category one show that knowledge means different things to different practitioners working in different contexts. Hence, the evidence quality depends on what we want to know, why we want to know it and how we envisage that evidence being used within the context of it being implemented. My analysis of data for this category show that there is an issue about how to define EBPs and according to whom, this is evident in Phil's extract, 'what constitutes evidence'. Steve's extract he seems to be highlighting the need for courses and supervision. Encapsulating EBP is complex particularly in light of patient values, experiences and preferences.

Interlinking the amalgamation of individual values, experiences and choices into evidence based practice is complex issue because what might be scientifically proven by science, for example, a good quality RCT might not necessarily fit well with the patient choice (Rycroft-Malone, Seers \& Titchen, et al., 2004). Hence having the skill and ability of the practitioner in eliciting these issues and negotiating the most fitting course of action is key to improving patient outcomes. 
The claims presented by Richard, Phil and Steve show what may be regarded as some of the important factors to sustaining evidence based practices in early intervention in psychosis teams.

To summarise category one there is a notion of what evidence based practice means and what it constitutes and to base practice knowledge upon what works. There is an underlying assumption that RCTs are the gold standard, but NICE don't have the control of what constitutes evidence based practice. There is a suggestion that guidelines are not disseminated sufficiently so that practitioners can stay up to date and a need for a discussion of how this happens to ensure a systematic way of dissemination.

\subsection{Category 2: Specialised Practice}

This category consists of a collection of research interview data that refers to the values, care and philosophy of early intervention in psychosis. Specialist practice is seen as an important part of the theme because participants argue or make claims on its behalf.

Sustainability is key to the implementation of a desired outcome, (Proctor, Silmere \& Raghavan, 2011) and a priority topic in implementation science, (Glasgow \& Chambers, 2012). The science of implementation focuses on the question of how we can effectively and efficiently get people to use research results and evidence based practice and programs in a consistent way. This may include the adoption of clinical best practice by service providers, also, the implementation of processes in facilitating the acceptability of intervention in communities.

\section{'Lack of understanding amongst the managers': advancing the case for special knowledge}

A common pattern in the data used for this thematic analysis was that participants were describing a 'lack of understanding' amongst managers. The managers that Jeremy refers to in the first data extract below are external to the early intervention in psychosis team; these are managers who are 'corporate' 
who make clinically strategic decisions on behalf of the NHS Trust. The 'lack of understanding' is captured in the first two research data extracts below by Jeremy and Geraldine. The third data extract from Carolyn demonstrates a different strand from an early intervention practitioner in that she advances a claim that others don't understand their work or the evidence base supporting it.

The data extract I present below is taken from a research interview with Jeremy who is a nurse consultant who works in a suburban area, southern England that covers six early intervention teams. Jeremy's team has been established for eight years with the main service model used in his service being a stand-alone specialist team with case management being the main type of service provided. Jeremy has sixteen years' experience of working in mental health services. Jeremy talks about managers who make corporate strategic decisions that have repercussions that have direct effects on early intervention team sustainability. $\mathrm{He}$ is referring to specialised practice in early intervention teams. The question asked to Jeremy was, 'what are the critical factors to sustaining evidence based practices in your organisation?'

"Well I would say that the main one is lack of understanding amongst the managers who make more corporate or clinically strategic decisions for the trust. I don't think they generally have a good enough understanding of day to day practice in the trust but certainly not specialist practice in the early intervention teams (meaning EIP). So they often send out edicts that clearly compromise the way we can and can't work". (Jeremy)

The philosophy of specialist early intervention in psychosis teams is a multidisciplinary community mental health service that provides treatment and support to people experiencing or at high risk of developing psychosis (IRIS, 2010). The ethos of early intervention teams of intervening early and effectively in the course of psychosis can limit initial problems and improve long-term prospects for recovery through the provision of individually tailored, evidence based interventions and support to service users and their families and carers (NICE, 2014).

In Jeremy's quote he makes a confident claim that people making decisions are not cognisant of actual service delivery and his reference to 'edicts' invokes the 
idea that decision makers are not collaborative and involving, but rather can be positioned as dictatorial in their judgements about service needs. Jeremy implies that this then challenges how teams deliver evidence based interventions and may actually disrupt the face to face delivery and experience of this care.

The data extract below is taken from a research interview with Geraldine who is a consultant psychiatrist in EIP who works in an urban area of England. Geraldine's team has been established for seven years with the main model used in her service being a standalone specialist team with case management and inpatient services as the main types of services provided. Geraldine has had more than eleven years' experience working in mental health services. Geraldine advances a claim that there is a lack of understanding of what EIP teams' do and as a result some ignorance of the specialist knowledge that EIP brings.

"I think there is quite a lack of understanding of what we do. Um so when lots of services are being disbanded there was a quite a lot of talk about, 'I don't know why they don't get rid of EIP, I don't know what they do'. You know they would have been better off working ... keeping assertive outreach. And getting rid of EIP and I think that's because what we do we do well. So the inpatient consultants don't see our patients cause we keep them out they don't know who we keep out. And um that we don't use up the crisis service. So I think you know there is a degree when doing things well that nobody knows what we are doing because it doesn't impact on them". (Geraldine)

In Geraldine's quote she talks about organisational and restructuring changes within her service when she refers to, 'services being disbanded'. She refers to her team doing well because the consultants that work in inpatient wards do not see EIP clients; hence adherence to the EIP model must be high. Effectiveness of EIP is dependent on the fidelity of the model, and changes to the model can impact on quality, care and outcomes. She claims there is ignorance from the people that commission services in wanting to get rid of EIP; this echoes the importance of the principles of EIP and what it does. 
At the outset of this data extract Geraldine introduces what she sees as threats, 'a lack of understanding' and 'lots of services are being disbanded' and suggests that there is wider discussion on the value and continued presence of the EIP services. Her counter argument is that this discussion and potential threat is based on a lack of knowledge and stems from the success of the service, 'what we do we do well'. She then reinforces this claim of doing things well with examples, 'inpatient consultants don't see our patients' and are unaware of the people who never get admitted because of the good work of the EIP and also 'we don't use up the crisis service'. Geraldine again reiterates that what they are doing they are doing well and this gives her argument a sense of strength and a difficult to dispute quality. The account reads as well-rehearsed and perhaps suggests that in the light of cuts to other services the EIP staff have got their arguments well prepared.

It is interesting that Geraldine like other participants in specialist services consistently complain about a lack of understanding of their work, but shows no effort or attempt to bridge the gap in understanding. It appears that Geraldine expects others to do the work of finding out about the EIP service rather than communicating the value of the work herself. Geraldine refers to, 'so the inpatient consultants don't see our patients'. This is interesting in that one would imagine a service under threat would collect outcome data on their service, and be able to present this to senior colleagues to show how successful they have been relative to other services. It is perhaps a key element of the role of EIP to intervene early and delay or prevent the need for hospital admission and Geraldine is advancing this argument along the lines of 'we are so successful we are anonymous'. This is an argument that has its limitations and services might be keen to celebrate such successes. In a time of economic austerity other services might be doing things just as well perhaps and yet still come under threat so Geraldine is showing awareness of the precariousness of current service provision even when evidence based and producing good results.

The data extract below is taken from a research interview with Carolyn who is a cognitive behavioural therapist in Wales. Carolyn's team has been established 
for six years; the service model used was a hub and spoke model with case management and inpatient services being the main type of service provided. Carolyn has more than six years' experience working in mental health services.

"I go back to the rationale for early intervention, and are people on board with it. In my experience, there's been a lot of staff, a lot of managers that don't agree that early intervention is needed, that felt that standard services were doing okay and why do we need this, why do we need to work differently. I found that as being quite a big issue. I don't think that people have a very clear understanding of what early intervention (meaning EIP) is about, exactly what we do and, more importantly, why we do it. That's been my experience". (Carolyn)

Carolyn is advancing a claim that others don't understand their work or the evidence base supporting it. These others who are referred to as 'a lot of staff, a lot of managers' are positioned as advancing an alternative version implying that, standard services can do the work and EIP services are working in a different way and is being challenged. Carolyn argues too that ignorance of the evidence leads to these attitudes. In doing so Carolyn positions EIP as requiring a special knowledge set that is absent even among other managers in mental health settings. It seems however, that in saying that others do not agree that EIP is needed, these managers must be using some knowledge themselves. My reading of this data extract is that it indicates the presence of the contested nature of service organisation and delivery in which the evidence itself is up for contention and claims and counter claims abound. This becomes important for services that might be smaller and on the periphery especially as cost cutting can mean their service could be easily removed as suggested by Gareth in his quote below.

"The current financial economic climate, however you want to describe it, that's been a challenge for El (meaning EIP) services and l've seen - this isn't the first early intervention service l've worked in I guess. I've seen that around the country, where they've been disbanded or been gotten rid of, they've just been absorbed back into CMHTs". (Gareth)

The data presented indicates that practitioners advance claims of the specialist nature of the work they do and that this requires specific awareness of the 
evidence base to enable decision makers and others to fully understand the role of these types of services. Traditionally there has been a lack of transparency between clinical and managerial work with little understanding between clinicians and managers. Ackroyd (1996) argues that clinicians, as professionals have occupied encapsulated enclaves within medical organisations and have assumed a narrow field of focus, thereby differentiating themselves both from senior executive management and routine operational management. Clinical directors as two way windows are seen as mediating persons as they work through sets of ideas belonging to management and sets of ideas belonging to clinical practice (Llewellyn, 2001). The two way window implies greater communication between practitioners' and management. These two domains (medicine and management) previously blurred into each other (previously formed as 'one way windows') become communally visible through the activities of clinical directors because they occupy boundary roles and Janus thinking - the ability to join two sets of ideas.

These communities of knowing have developed different logics; clinicians have been guided by the logic of appropriateness and managers have operated according to a logical consequence (March \& Olsen, 1976). In advancing the case for special knowledge the managers may be drawing upon some other forms of knowledge to aid their decisions about commissioning services. Messages from clinicians have tended to make sense to other clinicians and vice versa with managers which are known as the 'one way window' encounter (Llewellyn, 2001).

It is inevitable that however well innovations are supported by science they are always influenced by cost priorities and much debate concerns around the costbenefit analysis (Fitzgerald \& Dopson, 2009). Participants are communicating that managers that are making decisions about commissioning services don't understand the evidence for early intervention. One of the critical debates is in relation to who makes the decisions when adopting an innovation. The bearing of power between clinical hybrid managers (clinical professionals turned managers) and general managers is likely to have influence on decisions which in my view can influence adoption of innovation. In turn, defining roles and 
responsibilities of clinical managers and general managers is fundamental. This becomes more evident in the next section covering category two 'champions'.

\subsection{Category 2: Champions}

The category of 'Champions' refers to analysis of research interview data that show how individuals or a group who adopt a role of promoting and defending specialist services are considered important at sustaining early intervention in psychosis teams. This category links to the subcategory of senior management because opinion leaders can play a creative role in organisational settings to initiate change. In this section, I will show via analysis of research interviews how the champions' role is positioned by participants as influencing and articulating the effectiveness of the service and is considered crucial to the ongoing sustainability and funding of early intervention teams.

\section{'Commitment from some people - sort of champion of it...': initiating change}

Participants described that in order to sustain early intervention teams there is a need for commitment by certain groups and individuals who are established as being champions of the service. The manner in which evidence is communicated to key stakeholders is argued as being critical to championing EIP services.

The data extract presented below is taken from a research interview with Nigel who is a consultant clinical psychologist who works in an urban area of Wales. Nigel's EIP team has been established for a period of one year. Previous to the team being set up, there was no dedicated first-episode service; hence the team developed a pilot project within the CMHT. The main model of service is an enhanced CMHT with case management being the main type of service provided. Nigel has had sixteen years' experience working in mental health services. 
"I think there's also been a commitment from some people working within the health board to develop this sort of service, you know, sort of, champion of it". (Nigel)

In this extract from the research interview with Nigel he is arguing that even for services with a very good evidence base it requires some political support within organisations to get these up and running. Although he doesn't say so directly it does appear that clinicians really need to engage and make connections with like-minded individuals at board level who are open to hearing and acting on evidence for change in provision.

The data extract presented below is taken from a research interview with Steve who is a nurse specialising in psychosocial intervention care, working in Wales. Within the service that Steve works in, there is no early intervention in psychosis team; he works within a CMHT that has been established for some eleven years and it is this team that first encounter referrals of first episode psychosis clients. Steve has worked in mental health services for over sixteen years. In this data extract Steve argues that champions within teams are important to sustaining EIP teams.

"We've had good champions like the [clinical psychologist] in Wales. He was brilliant at maintaining enthusiasm for the first episode network in spite of getting all sorts of mixed messages from senior management. He was very good at maintaining optimism". (Steve)

Steve refers to a colleague and how he was an excellent champion who maintained enthusiasm for first episode networks. Steve suggests that noteworthy qualities are important for instance I note the use of 'enthusiasm' and 'optimism 'which are individual qualities that he suggests his colleague has for maintaining interest and the pursuit of early intervention. Scheirer (2005) evaluated 19 empirical studies of sustainability of American and Canadian health related programs examining the extent of factors that contributed to greater sustainability. Of the total of nineteen studies included, thirteen emphasized the important role of a program champion; this person may be strategically located, having access to upper management as well as having influence on, or control over, day-to-day program operations. The champion is 
often an enthusiastic advocate for the needs of the program, particularly to secure resources for its continuation. Having a champion, a key influencer is paramount where say, funding may be a problem, and, their influence would be a key factor in sustaining its long-term existence. Aarons and Sawitzky (2006) argued that having a positively perceived local opinion leader who can influence organisational culture and who can guide change in practice may facilitate receptivity to change in provider behaviour (Aarons and Sawitzky, 2006, p68). McGorry and Yung (2003) state that greater coalitions for investment in EIP can make a difference as seen in an approach used in Australia. McDaid and Kapp et al., (2016) suggest that effective advocacy for the presence of champions of EIP, whether these are: leading academics, clinicians, and institutional structures such as professional associations or nongovernmental organisations.

In looking at the importance of system level change, including support from the upper levels of management and support from clinical staffs' immediate line managers: Dopson, FitzGerald, Ferlie, Gabbay and Locock (2012) highlight the importance of 'opinion leaders' to the implementation process. Chambers, Surender and Dopson et al., (1999) argue that such advocates bring credibility and establish leadership within their own professional groupings. One significant finding across promoting action of the clinical effectiveness initiative was the importance of opinion leaders to the implementation process (Locock et al., 2001).

There is no doubt that, the role of clinical leaders' opinion is complex. The evidence from Chambers, Surender and Locock et al., (1999) study tends to suggest that there is an effect, but, it is part of a wider process and cannot be seen in isolation from other contextual variables, with which it may interact. It should also be noted that the effect is not always positive, in the sense of supporting the desired change the influence of hostile or ambivalent opinion leaders is an important and neglected area. Chambers, Surender and Locock et al., (1999) have therefore adopted the use of the term opinion leader as opposed to the narrower term change champion. Whatever the exact mechanism by which opinion leaders exert influence; their active support for and involvement in a particular initiative is powerful. 
In Steve's extract he refers to a colleague who was a good champion and maintaining enthusiasm for the first episode network. Steve refers to a network which I argue functions in a similar way to a community of practice which can be seen as a mechanism to exert influence. Communities of practice are defined as a group of people who share knowledge, learn together, and create common practices (Wenger, McDermott \& Snyder, 2002). Communities of practice are wrought by three fundamental elements: domain of knowledge creating common ground, identity and inspiring members to contribute and participate (Barwick, Peters \& Boydell, 2009). Connected to the category of champions, was a subcategory of senior management and this subcategory is discussed below.

\subsubsection{Subcategory 1: Senior Management}

The subcategory senior management is connected to the category of champions because like champions senior management can influence change and act as agents and make a case for investing in EIP. Participants advance that having a clinical director who also works 'on the ground' is able to understand the challenges and day to day practice EIP brings.

\section{'Our clinical director was working with us for well over a year in a clinical capacity': the two way window, the privilege of boundary roles}

Within the category of champions senior management is a common reoccurring theme, a subcategory - senior management was a feature in a number of research interviews with participants. Senior management is related to champions because participants provided examples of positive involvement of senior management in supporting, and recognising the work of other services. In the data extracts presented below Martin and Richard describe a situation of a clinical director who was working in the team that had senior responsibility at management level. The sub category senior management links back to the 
theme sustaining EBPs as participants viewed this as critical feature of sustainability because they are seen as change agents.

The first data extract below is from a research interview with Martin who is a team manager in an urban area of London. The early intervention in psychosis team that Martin manages has been established for four years. His early intervention team is a standalone specialist team which provides case management as the main service model. Martin has worked in mental health services for sixteen years. He advances the claim that, having a clinical director who is also a clinician working within the early intervention in psychosis team helps to disseminate the day to day working practice of the team.

"Our clinical director was working with us for well over a year in a clinical capacity, within our trust - clinical director is expected still to practice one day a week. Ours is a psychologist by background, she was working with our service users and within our team. I think that helped enormously for her to understand what we do, and the value of it and the demands and challenges". (Martin)

I suggest that Martin is stating that in having a person who operates on a management level who is a clinician they bring together the clinical expertise along with the management responsibilities collectively. This can be seen as a powerful alignment for the organisation because there are two sets of role expertise that Martin's colleague can bring. Hence the metaphor of the two way window is apparent here in that a clinical director who is also a practitioner is able to operate, and explore the states of the 'in-between-ness' (Learmonth, 1999). The 'in-between-ness' is a metaphor which is employed to encourage thinking outside accepted paradigms commonly used to examine management. The in-between-ness leads to new understanding that, in turn can lead to creative action.

The data extract below is from a research interview with Richard who is a consultant psychiatrist in an early intervention team in England. Richard's team has been established for eleven years with a hub and spoke model used within the service with case management as the main service provided. Richard has been working in mental health services for sixteen years. Richard emphasises 
the importance of having a clinical director who also works in EIP keeping the matter well up on the radar.

"One of the most important things for us is having a clinical director who works in early intervention and who has been able to keep it on the radar". (Richard)

The data presented above are testimony to the view that some importance is attached to having senior people working within EIP teams, they gain valuable understanding of the mind-set and philosophy of EIP teams. Moreover, the knowledge gained can be effectively communicated back to key decision makers or commissioners. Power (1994:12) introduces the metaphor of the 'two-way window' which encodes information suggesting increased interchange. Clinical directors are positioned in a privileged way because they can operate as the two-way window (Llewellyn, 2001). McCasky (1998) states that, they exhibit 'Janusian' thinking: this is the ability to constructively join two sets of traditionally contrasting ideas. I acknowledge that this might not necessary be the case universally, but this can be advantageous.

\subsection{Summary of Theme One}

My analysis from theme one shows the important factors to sustaining evidence based practice in early intervention in psychosis services as articulated by practitioners in those services. Evidence based practice is not a 'one size fits all' approach to treatment. Nor it does not imply that practitioner knows best for the client. Furthermore it implies careful collaboration between client and practitioner by which both make decisions about treatment in the light of goals and preferences.

A common pattern in the data was that participants were stating that there was a lack of understanding amongst the managers in relation to specialist practice which might be an issue for future skills that early intervention practitioners hold. This finding was also found in Court, Cooke and Scrivener (2017) where they interviewed clinical psychologists about beliefs about NICE guidelines. Participants were concerned that there was an increasing dependence on 
guidelines, predominantly by managers and commissioners without specialist knowledge.

Champions and senior management were seen as agents that could initiate change, who knew the evidence for early intervention. Hybrid managers (clinician with senior management responsibility) were seen as initiating change at more upper management level, who knew the knowledge for EIP and were able to make a good case for EIP to decision makers and commissioners.

I advance the case in theme one of bringing about change to achieve evidence based practice and basing knowledge on what works. Bringing change is expected to emphasis systems thinking which might adopt (micro and macro approaches). These approaches and constituent parts are brought together in a reciprocal relationship (Nutely, Davies \& Powell, 2003).

In the next theme I will discuss challenges and opportunities which consist of two categories of barriers and protection of early intervention in psychosis services.

\subsection{Theme 2: Challenges and Opportunities}

This theme addresses research questions two and four which are, 'To what extent is service context and culture relevant to practitioner's experiences of adopting evidence based practice?' and what do early intervention lead practitioners say are the facilitators and barriers to implementation of evidence based practices in their services?' This theme collects together claims made by participants in which they construct their versions of challenges and opportunities to implementing evidence based practices in their services. This theme includes two categories of 'barriers' and 'protection of early intervention services'. The barriers include four sub-categories which are (1) fidelity (2) funding (3) practice proficiency and (4) individual attitude. The second category of protection of early intervention services includes one sub-category which is outcome data. The theme and categories are displayed in the visual representation below in Figure 19. 
In the following sections, I will show how the theme of 'challenges and opportunities' is constructed of multiple categories that focus on claims of barriers and protection of early intervention services to adopting evidence based practices in early intervention services.

Most implementation frameworks attempt to identify barriers and facilitators to practice change. Facilitators and barriers can be categorised as two types of attributes - attributes of individuals and attributes of innovative systems.

Attributes of individuals identified by Greenhalgh, Robert, Macfarlane, Bate \& Kyriakidou (2004); \& Rogers, (2003) that contribute to successful adoption include: relative change, compatibility with current norms and values, low complexity, trialability, observable benefits and flexibility in the setting. Attributes of innovative systems include decentralized decision-making, diverse professionals with specialized knowledge, lack of formality, good internal communication, and technical support for change (Damanpour, 1991).

\section{Figure 19}

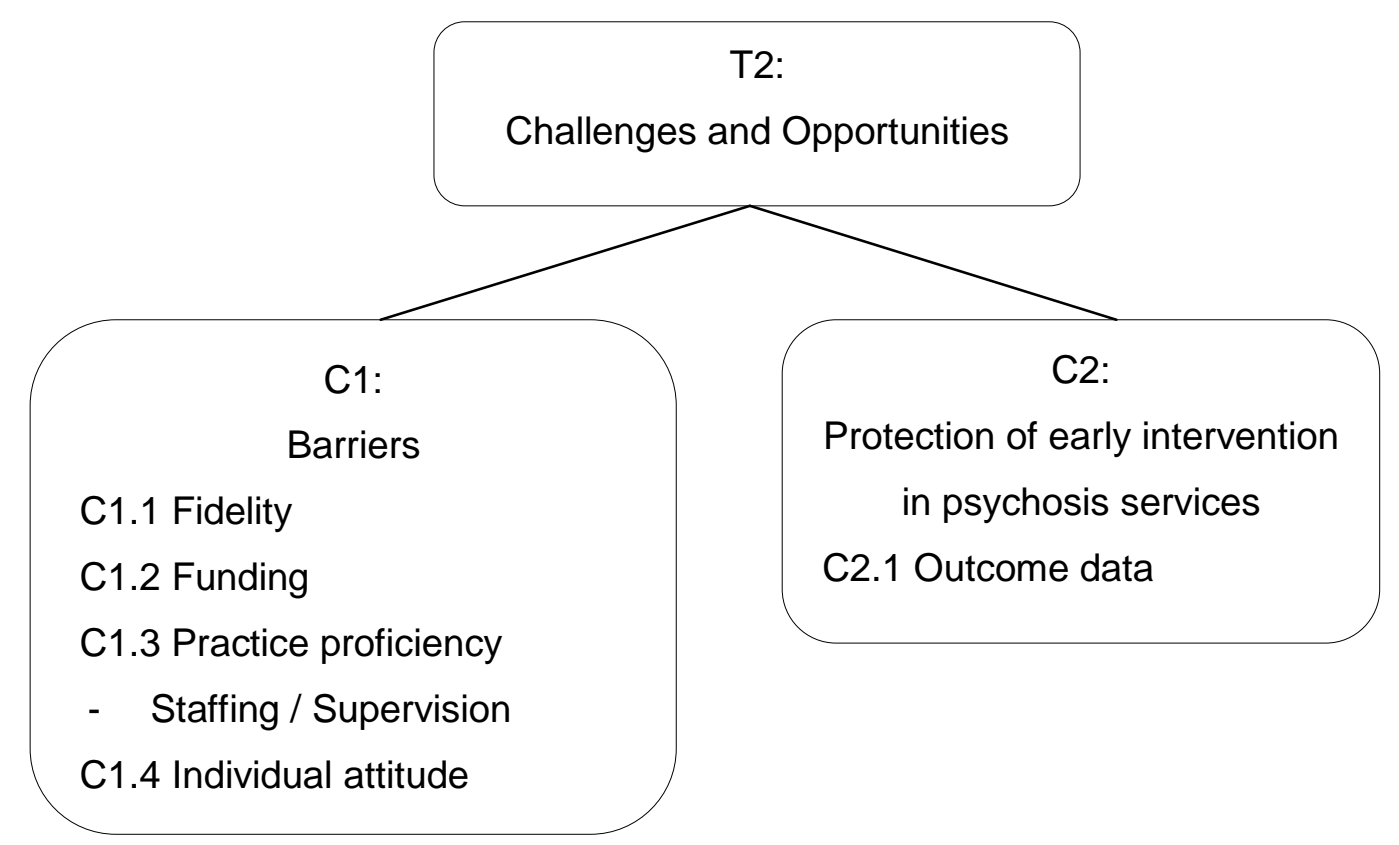

Figure 19: A visual representation of theme and categories $\mathrm{T}=$ Theme; $\mathrm{C}=$ Category 


\subsection{Category 1: Barriers}

In this category I collect together participants talk on the barriers their organisation faced with regards to implementing evidence based practices in their services. Another way of looking at this is, 'what are the obstacles in implementing EBPs in their organisation?' I will show in this category of 'barriers' how participants implicate the issues of fidelity, funding, practice proficiency and individual attitude as potential obstacles to the delivery of evidence based practice in early intervention services.

The theoretical framework by Aarons, Hurlburt and Horwitz (2011) indicate that the adoption and implementation process is multilevel with many challenges influencing how evidence based practices are successfully implemented at different stages over time.

\subsubsection{Fidelity}

Fidelity is defined as the degree to which an intervention was implemented as it was prescribed in the original protocol or as it was intended by the program developers (Dusenbury, Brannigan, Falco, Hansen, 2003 \& Rabin, Brownson, Haire-Jushu, Kreuter \& Weaver, 2008). The concept of implementation fidelity is defined in the literature in terms of five elements that need to be measured (Mihalic, 2004; Dane \& Schneider, 1998), however examining what people say about the introduction of evidence based practices may also help us tease out and understand the nuances of how fidelity is implemented or becomes an obstacle in achieving the aims of early interventions services. The elements of implementation fidelity are: adherence, quality of delivery, program component differentiation, exposure to the intervention, and participant responsiveness or involvement.

Qualitative research interviews can explore in-depth phenomena or detailed insights provided by individual participants which quantitative methods cannot achieve. This in turn helps us to understand the processes in which the context of the sustainment an EBP can prevail. If implementation adheres completely to the content, frequency, duration and coverage prescribed by its designers then 
fidelity can be said to be high. I think that having adherence all to be high in content, frequency, duration and coverage might not always be desirable and may work to exclude some people from services where there needs are not met by services providing a one size fits all approach.

Fixen, Naoom, Blase, Friedman \& Wallace (2005) say that prevention programs shown to be effective in clinical trials may not impact the health of society unless they are delivered with fidelity.

Drake, Goldman Leff et al., (2001) note that if two programs offer a practice of care that is effective, the program with higher fidelity to the defined practice model tends to produce superior outcomes. This is an important finding which contradicts the conventional wisdom that model programs do not transfer and need to be modified extensively to fit local circumstances (Drake et al., 2001).

Fidelity is important because it refers to the degree which particular programme follows a program model, hence well-defined interventions and procedures to help individuals achieve some desired goal (Bond, Evans, Salyers, Williams \& Kim, 2000).

\section{'...real world clinical practice sometimes requires deviation from models': the role of context in the implementation of EBPs}

A common pattern in the data was how adherence to the EIP model was constructed by participants and how the notion of fidelity was a barrier for practitioners. I was surprised by this as fidelity is considered key to delivering interventions that are manualised or part of a protocol. The patterns are reflected in three data extracts from research interviews I have selected and analyse below.

In a data extract from a research interview with participant Anne she describes caseloads and how this is important in terms of the EIP model fidelity. Another participant Phil describes how real world clinical practice requires deviation from 
models because service users don't fit the prescribed manual. Participant Huw describes how fidelity can be time consuming for example in the use of audio tapes of interventions and the time needed to score them. These three extracts encapsulate the issues raised by fidelity in their unique ways. Participants discuss model drift and they indicate some loosening of definitions and applications of fidelity.

The data below is taken from a research interview from Anne who is a consultant psychiatrist who works in a suburban area of South England. Anne's team has been established for ten years with the main model used in her service being standalone specialist team with outpatient and case management being the main types of services provided. Anne has eleven to fifteen years' experience working in mental health services.

"But, in terms of sort of model fidelity, yes there is, I suppose you can call it kind of fidelity creep. So, as I have explained the caseload for each care coordinator has gone up from 15 to 20 now, you know that was both IRIS and Policy Implementation Guide suggested that care coordinators should have a caseload of 15 now. Whether you would call that actually based upon robust clinical evidence, it is hard to say. But what we do know is that once it goes sort of certainly up to kind of 25, 30 then you are operating like a CMHT team; and the assertive approach, a lot of the approaches that run hand in hand with operating early intervention just go by the wayside". (Anne)

Anne is describing EIP model fidelity in relation to adherence indicator of 'caseloads' within her team. Anne is advancing a claim that EIP caseloads should remain low and with any increase above 15 people on caseloads the model fidelity is lost and hence the team is operating more like a CMHT than an early intervention service. The effectiveness of EIP services is dependent on the fidelity of the model and changes to the model can impact on quality of care and outcomes (Breitenstein, Gross, Garvey, Fogg \& Resnick, 2010). Anne makes reference to polices such as Policy implementation Guide and IRIS and that care coordinators should have caseloads of 15. When Anne makes reference to IRIS she is referring to the initiative which was the inspiration behind the ground breaking reforms scaled up across England which is seen as a standard feature of mental health care. 
An important ingredient of an effective EIP team is a low caseload which should equate to no more than average than 12 to 15 cases per key worker (IRIS guidelines, 2012). The case load size is important and if it goes over 15 the adherence to EIP model is not being accomplished. In a qualitative study involving interviews with a wide range of health commissioners, EIP team members and service users Lester, Birchwood, Bryan, England, Rogers \& Sirvastava, (2009) suggests that team size can vary with key workers expected to manage an active case load of 15 cases, much lower caseloads than in conventional community mental health teams.

Lester et al., (2009) also found that a major barrier to developing services from an EIP perspective was indecisiveness over funding due to commissioner inexperience to lack of understanding the ethos, delivery of services leading to a delayed decision-making, and problems securing ongoing funding. A recurring pattern in the interviews was that most team managers particularly the newer teams stated that commissioners were too focused on ensuring that case-load of 15 patients per key worker was achieved, which was connected in part to securing the following years funds.

Maintaining an active caseload is an important adherence indicator for EIP as Amanda states in a data extract from a research interview below because she highlights the fact that interventions may take more time to deliver due to the very nature of their specificity. Amanda is highlighting the pragmatics of a low caseload as it is not possible to see more people than this on a regular basis when interventions and travel take so much time in a working day.

"You might have a caseload of 15 which is roughly a low caseload. You might only see two to three people a day because your interventions are more specific, more timely and the distance travelling between them eats into your day, so that's another challenge". (Amanda)

The issue of geography was also found in Lester et al., (2009) study where the influence of geography was felt more in rural services, where balancing travel time and face-to-face work with service users was a constant issue. 
The data extract below is from a research interview with Phil. He describes how the team haven't used fidelity assessments over the last couple of years. He then explains how resources in terms of staff time are a barrier to implementing EBP. He speaks about how real world clinical practice requires deviation from the manual and that flexibility is required for the client group.

"I guess it's true over the past couple of years we haven't used by fidelity assessment you mean things like the cognitive therapy rating scale? "We don't use anything like that not routinely anyway there may be occasions when people are doing particular courses that is used, but I would say there is a number there are two reasons obvious reasons that come to mind it's the resourcing question the kind of thing that takes extra time and ends up being neglected, but another thing I think real world clinical practice sometimes requires deviation from models because service users don't fit and sometimes the therapy doesn't fit as it might be prescribed in a manual. There's a requirement for flexibility in working which might actually mean that not that focusing on fidelity limits the flexibility for working that is required for this client group". (Phil)

Phil refers to 'flexibility' in working with clients as they don't fit or the therapy doesn't fit as prescribed in the manual. There are two important aspects in Phil's data extract which is the 'flexibility to adapt' the intervention within real world clinical practice, and drift from consensus on how best to deliver interventions. Phil is advancing a claim that real world clinical practice does bring its challenges when he mentions 'resources' and deviation from models.

In many early intervention in psychosis teams the standard practice is to measure fidelity through self-report, ratings, and direct observation and coding of audio and videotapes of actual encounters, or provider-client/patient interaction. Achieving and measuring fidelity in usual care is beset by a number of challenges (Proctor et al. 2009; Mihalic 2004; Schoenwald et al. 2005). The foremost challenge may be measuring implementation fidelity quickly and efficiently (Hayes, 1998). I acknowledge actually achieving and sustaining fidelity would be a greater challenge then measuring it. This can be seen in Huw's data extract below on the day to day challenges with fidelity. 
The data below is taken from a research interview with Huw who is a clinical psychologist who works in rural North West England. Huw has six to ten years' experience working in mental health services. The main model used in his service is hub and spoke with case management and day treatment being the main types of services provided. Huw notes that achieving and sustaining fidelity requires significant amounts of time to do this regularly. In services that are pressured clinical supervision is usually the first thing sacrificed or suspended when time is short and the type of supervision required to sustain and check fidelity with audio tapes of sessions being reviewed would take longer than usual. He refers to scoring the tapes and seems to be taking it a step further again and the reference to it being a cultural thing suggests it fits with certain types of service delivery and not others.

"I have used them (tapes), but the reason why that's not ongoing, I think, is... There's a range of reasons, I think some people are more likely to take questions to supervision and focus it on that, rather than listening to tapes and scoring the tapes, so I think there's a cultural thing that makes that sometimes difficult, but also I think just the time that it takes to listen to a tape of a session and score it, given just the many challenges. Certain aspects of performance there are fidelity scales which can help, but the time taken to do them, score them, record them, and feedback is another factor that needs to be made against the many challenges that we have just to keep the service going. Whilst that's a facilitator of fidelity, I think it's with increased challenges on time and that's one of the first things that can go". (Huw)

Although manual based treatments are not synonymous with evidence based practice, they do provide a potentially helpful means to utilize efficacious interventions in real world clinical settings (Addis, Wade \& Hatgis 1999). In a paper by Addis, et al., (1999) the immediate issues are explored around the practical, psychological and systemic variables that directly affect practitioners' ability to use manualised treatments. The authors discussed practitioner's common concerns such as effects on therapeutic relationship, unmet client needs, competence and job satisfaction, treatment credibility, restriction of clinical innovation, and feasibility of manualised based treatments.

Addis (1999) says that using a treatment manual is not a matter of theoretical or empirical debate for practitioners. It's a psychological reality. He explains that in 
using the term 'psychological reality' it is the practitioners that are the ones who must grapple with attitudes, feelings regarding autonomy, competence and the perceived threat of manual-based treatments. The economic and political context in which evidence based practice is another important psychological reality. Over the last 20 years practitioners have begun to feel the direct effects of the economic and accountability contingencies and will continue to do so. More than ever, clinicians must answer questions about why they're treating particular clients, why they're choosing a particular intervention and whether such choices are justified economically in terms of outcomes. The context is important because it's where the proposed change is to be implemented. EBPs are complex and multi-faceted interventions that are difficult to implement without some level of pre-existing structure and support (Torrey et al., 2001). This category captures the notion of the context in which EBPs are implemented in EIP teams, it is important to understand the environment in which EIP services are delivered as clinical reality seems far apart from manuals due to resource implications.

\subsubsection{Funding}

Practitioners described how funding was seen as a barrier when faced with implementing evidence based practices in their organisations. There are increased challenges in regard to financial pressures on services for EIP provision in England and Wales (Docherty \& Thornicroft, 2015). Even where the availability of services are relatively good, the present economic climate places substantial strain on services (McDaid, 2016). Community services have undergone considerable reconfiguration in recent years including remodelling, decommissioning and integration, for example the majority of assertive outreach teams have been dismantled, with some functions integrated into community mental health teams (Firn, Hindhaugh, Hubbeling, Davies, Jones \& White, 2013).

Early intervention in psychosis teams are distinct due to their function, but many have been integrated back into CMHTs (National Confidential Inquiry into Suicide and Homicide by People with Mental Illness, 2013). The data extracts 
below are from participants who construct their meanings of how funding was seen as a barrier to implementing EBPs.

\section{'I suppose NHS cuts would be the other thing: lack of protection for EIP budgets}

The data extracts below are from research interviews with Jeremy, Gareth and Rob. Funding of EIP services is a barrier to adopting EBPs along with the way current standard services operate and apply treatment as usual to EIP. Jeremy describes how they experienced a lot of political and economic pressures to establish EIP services. Gareth describes how funding cuts have affected the establishment of EIP teams and recruitment and retention issues that they have experienced in relation to the medical time allocation. Rob on the other hand explains that they do not have a fully-fledged EIP team; theirs is only a pilot project. Funding is limited and also protection of staff time to deliver interventions has been a barrier.

The data below is taken from a research interview with Jeremy who is a nurse consultant. Jeremy's team covers six early intervention in psychosis teams in the south of England. He is describing how their service had a lot of financial and economic pressures to establish an early intervention in psychosis service. Jeremy describes how financial pressures were imposed on the team to establish an EIP service. He refers to the calibre of staff that were recruited as early intervention in psychosis staff are required to be highly skilled to deliver interventions. He refers to 'principally established services' wanting to apply treatment as usual to an early intervention population.

"We had an awful lot of financial pressures put on to us to establish an early intervention service(meaning EIP) across the six teams from what would have been the equivalent of the strategic health authority back in the day. So there was some considerable political and economic pressure to get it up and running, but what's actually maintained it or what's realised it I think has been the calibre of the staff who were initially recruited. I don't think there's ever been any that have actually threatened El (meaning EIP), but they've made it more difficult. They are principally the established services, particularly established medical services wanting to 
apply treatment as usual to an early intervention population. It doesn't work and might be contra-indicated, so sort of standard or high dose medications, inpatient units that are designed for general populations and not for first episode or young people". (Jeremy)

Jeremy refers to the CMHT which are the established teams that he asserts have made it more difficult to apply treatment to early intervention in psychosis population. Jeremy is advancing a view that the established services are operating very much like the medical model. He does this with reference to "high dose medications' and 'inpatient units'. Again it seems that the ethos, philosophy and evidence for early intervention are not understood. He mentions the qualities in staff who were initially recruited which highlights the importance of skilled and knowledgeable people which is similar to theme one category three of champions.

The following data extract is taken from a research interview with Gareth who is an early intervention psychosis team manager from a nursing background. Gareth works in an urban area of Wales. Gareth's team has been established for five years with the main model used in his service being standalone specialist team with inpatient, outpatient and case management all being the main services provided. Gareth has more than three years' experience of working in mental health services.

The question asked to Gareth was, 'please tell me factors that worked against sustaining early intervention in psychosis teams at your organisation'. Gareth refers to investing to save with EIP services. That is funding early intervention in psychosis to gain long term benefits. Funding has been a barrier to sustaining EIP as Gareth says. Gareth then advances a claim in relation to workforce issues such as 'lack of doctors'. Recruitment and staff shortages are positioned as current barriers hence allocating medical staff to CMHTs for early intervention in psychosis services. This can be read that early intervention in psychosis is not on the priority list of medical staff time allocation.

"I suppose NHS cuts would be the other thing. Again, you have to invest in early intervention services (meaning EIP) to make those services long term. The current financial economic climate, however you want to 
describe it, that's been a challenge for EIP services and I've seen - this isn't the first early intervention service (meaning EIP) I've worked in I guess. I've seen that around the country, where they've been disbanded or been gotten rid of, they've just been absorbed back into CMHTs. The current economic climate, I would say, that's been definitely a challenge. If you look at the evidence, or the evidence that l've seen certainly, incidents of psychosis increase when the economic climate worsens. That's been a challenge. The other thing has been, this is quite Wales specific now, but a lack of doctors applying for training posts in Wales, which means that there's a shortage of staff grade level, registrar level staff, doctors.

We've got a consultant, although his time his very precious. We only have him two days a week. Ideally we'd have a registrar or a staff grade level doctor but there aren't enough in the health board as a whole. That's one problem.

I would say we're probably a fairly low priority in terms of, or at least in the eyes of those people who allocate medical time to the different teams. The CMHTs are already struggling to get enough doctors. We're not first on the list to be allocated a registrar". (Gareth)

Mental health budgets have come under pressure in England, but obtaining accurate data to explore whether EIP spending has reduced is not easy. A survey undertaken by a leading mental health charity Rethink and the IRIS network of mental health professionals that support the promotion of Early Intervention in Psychosis (Rethink Mental Illness, 2014) stated that the services have reached tipping point, where critical additional investments are needed to maintain services, some teams have been disbanded and other subsumed into conventional community mental health teams. This is reflected in Gareth's quote above when he refers to EIP teams being disbanded and absorbed back into CMHTs.

The data extract below is taken from a research interview with Rob who is a nurse consultant in Wales. Rob's team has been established for one year with the main model used in his service being enhanced CMHT with case management being the main service provided. Rob has sixteen years' experience working in mental health services. They have a model which is only in the early development of delivering through generic CMHTs. The service has an organisational commitment to each generic CMHT which involves having a care pathway for people with first episode psychosis in which the team would adhere to the model within the service. 
"Obviously, like I said we haven't got funding for a fully-fledged team at the moment. This is just a pilot project, so we haven't got separate funding or anything else. Locally, there's limited investment in this initiative. There's no capital or recurring funds that are then ring fenced." (Rob)

Rob states that they do not have the funding for a fully funded service. They have a pilot project, so they are operating like a CMHT. Since October 2009, the 22 Local Health Boards (LHBs) and seven of the NHS trusts were replaced by a new structure made up of seven new Health Boards. The new health boards are responsible within their area for planning, funding and delivering of services. This is not usual in Wales where the service provision varies due to need determined by health boards and very few areas have Early Intervention in Psychosis services.

According to the Wales Audit Report (2005) in general, early intervention in psychosis teams and support outreach services are poorly developed across Wales. Very few areas have a dedicated early intervention in psychosis and treatment service or team. A number of Community Mental Health Teams (CMHTs) reported that they included early intervention in psychosis as part of their remit, and in one area the CMHT had identified and trained a limited number of staff to provide an early intervention service. Therefore early intervention in psychosis services provided from within CMHTs were unprotected and risked being squeezed out (Wales Audit Report, 2005, 1.39, p25).

In looking at service availability, the current economic climate places considerable strains on services. In England overall spending on adult mental health services has declined since 2011 after a decade of growth (Mental Health Strategies, 2012, Campbell, 2014, McNicoll, 2014). In England funds are distributed from NHS to local clinical commissioning groups who are then responsible for commissioning the majority of health services in their local areas. Thus, entering into a contract with one mental health trust for the provision of services. Consequently while the allocation of resources theoretically has included an adjustment for local mental health needs, funds 
are not earmarked CCGs and their former Primary Care Trusts have always had prudence on how much funding they actually devote to mental health services (McDaid, 2011). With this in mind this has led to considerable disparity in spending as noted by Campbell (2014) that some CCGs spend less than 7\% of their budgets on mental health compared with more than $18 \%$ in other areas. While variation in spending is inevitable the analysis found that in some areas the equal need and similar socio-demographic characteristics spending in one may be less than half of the other.

I am asserting a case here that the funding provision of early intervention in psychosis services delivery varies. This is evidenced in the claims that Gareth and Rob make above in relation to funding and staffing issues as seen in the 'lost generation' report by Rethink (mental health charity) which revealed that early intervention in psychosis services in England were struggling to survive in the face of major funding cuts. The survey found of 96 of 125 early intervention psychosis services in England 50\% had seen their budgets cut in the last year. More than half of early intervention services had lost staff (58\%) (McNicoll, 2014)

\section{'I guess one of the really important things is supervision. Supervision and availability of supervision': promoting a learning culture}

\subsubsection{Practice Proficiency (Staffing and Supervision)}

Participants viewed practice proficiency as integral to sustaining an evidence based practice which links back to fidelity. When participants talked about practice proficiency they included: staffing, supervision and training which in their view were seen as barriers to adopting EBPs.

Early Intervention in psychosis teams work intensively with young people experiencing a first episode psychosis. Gillam (2010) explains that this work presents challenges for team members. Amongst the excess of the demands practitioners may feel that there is little time for discussing their work in a 
considered way and little time to reflect on practice. Practitioners are expected to reflect upon their work and commit to lifelong learning to be constantly improving practice and ensuring it remains evidence based. Clinical supervision can be seen as a vital means of maintaining a healthy effective team.

There is variability in supervision across EIP teams across countries may be attributable to model difference, whereas within practice variation may reflect the level of resources. Cleary and Freeman et al., (2006) argue that successful implementation of clinical supervision can only occur when there is a 'learning culture' within the service hence a service wide commitment to staff development.

Participants constructed practice proficiency in terms of workforce issues such as staffing, supervision and training, which were barriers to adopting EBPs in their organisations. The definition of supervision did not vary much between participants. The frequency of supervision and the lack of supervision across participants was common. The process of accessing supervision in which client groups differed was a problem.

Huw's quote articulates his view of how important supervision is, and the availability of supervision to skill-up a workforce to deliver EBPs. In the data extract from a research interview with Nigel he advances that fidelity is an area of difficulty and he acknowledges that the team have attempted to address the issue in terms of resources such as supervision and clinical reviews.

"Yes, I guess one of the really important things is supervision. Supervision and availability of supervision, both people having time to take supervision and having people in place to provide that, is really important". (Huw)

"Yes. I mean, I think fidelity is an area of difficulty. We've attempted to address is by providing the team with adequate resources, and also by providing monthly supervision and regular review of the, kind of, clinical work with people". (Nigel)

The types of model that participants have in their organisation suggest profound effects on resource implications. For example, the hub and spoke model which 
Huw indicates operates in his organisation which is found in rural areas is difficult to support because the dedicated EIP team workers are based within more generic CMHTs and link to an EIP hub (IRIS, 2010) This can have subsequent effects on the staff that supervise the 'spoke staff' at a distance which quality may suffer because of this. The enhanced $\mathrm{CMHT}$ model that operates in Nigel's organisation is the least expensive model to implement which has least contact with people in similar roles (Fowler, Hodgekins, Howells, et al., 2009).

Without a dedicated Early Intervention in Psychosis leader it would seem that training and supervision will be difficult to implement. As such the fragility of EIP function demands a reliance on the wider mental health team - hence the CMHT culture will dominate. Swain and Drake et al., (2010) looked at factors affecting sustainability of EBP and supervision was viewed as integral to sustaining a practice. At some sites where multiple evidence based practices were implemented, group supervision was integrated with selected times for individual practices on a rotating basis.

The authors note that the variability of 'supervision time' across practice may be attributable to model differences, whereas within-practice variation may reflect the level of resources. A review of research by Freitas (2002) into the impact of clinical supervision on client outcomes found the field surprisingly sparse. A randomised control trial conducted by Bambling et al., (2006) found that clients being treated with psychotherapy for depression had better outcomes if their therapists were supervised than did those whose therapists were unsupervised. Bradshaw et al., (2007) found that nurses receiving clinical supervision showed improved knowledge of psychological interventions compared with peers who had not received supervision. Consequently the literature does show evidence to support the proposition that clinical supervision has an influential function that can benefit clients. This category of practice proficiency shows the importance of service context and organisational culture. Supervision and resources will promote implementation fidelity which is seen as facilitators but if these are nonexistent due to lack of resources then this makes implementation of EBPs difficult. 


\section{'I think that there's only so much that you can do in terms of changing a culture': assessing the readiness for change}

\subsubsection{Individual Attitude}

In this section my analysis will show how practitioners described how a person's individual attitude was a barrier to adopting an EBP. Work attitudes form an individual-level construct and most frequently include job satisfaction and organizational commitment (Glisson \& Durick, 1988). An important aspect of organisational culture is individual attitude as this can impact on how readily new technologies are considered and adopted in practice.

The two data extracts presented below are from research interviews with Huw and lan who were responding to the question, 'what things either in your control or out of your control that you think worked against sustaining EBPs at your organisation'? The two data extracts below address individual attitude in different ways. Huw's data extract encompasses culture change and individuals not wanting to change or adapting to change innovations. In lan's data extract he states that it's up to the individual to search for evidence in relation to EBPs via literature searching.

In Huw's extract below he advances the 'culture change' aspect of the organisation and how this was out of his control in implementing EBPs. He refers to the atmosphere and the climate in which people work and there being 'strong personalities' who are not motivated to address those cultural elements.

In an increasing need for data and measurement given the increased pressure on different services to justify themselves in a climate of cost improvements these are the cultural aspects that Huw is referring too that are out of his control.

"I think that there's only so much that you can do in terms of changing a culture as well, in terms of the atmosphere and the climate within which people work, and that if there are strong personalities who are not 
motivated by addressing those culture - those cultural elements, then that's quite difficult to manage and it can lead to disempowerment of staff and reduced morale, which will have a very negative impact on quality of care". (Huw)

Huw is saying that individuals can have a negative effect on the rest of the service such that cultural change becomes difficult to implement. He goes further to advance his view that this not only has negative effects on staff 'reduced morale' but more potently perhaps that it has a 'very negative impact on the quality of care'. So for Huw individuals who don't fall into line and engage with the agenda for changing service foci are seen as damaging both staff and service users. His view that there is 'only so much you can do' works to both express his frustration but also to some degree of releasing him of responsibility for any view that the service is not achieving what might be expected of it.

The data extract I present below is taken from a research interview with lan. lan is an associate specialist in psychiatry working in Wales. lan's team has been established for one year, with the main model used in his service, being standalone specialist with day treatment being the main type of service provided. Ian has sixteen years' experience of working in mental health services.

lan advances his claim in this data extract that it's up to the individual to keep up to date with developments with evidence based practices. Although he suggests that people use the excuse of lack of 'ideal resources' he sees this as no excuse and that libraries are well equipped to provide this service.

"Those things are totally out of our control, but talking about evidence based, I think that's down to the individual. We can't really complain that we haven't got the ideal resources, because you can go into the library and even if you have got technophobia or whatever go through anything, if you don't know how to go about doing a lit search or whatever. We've got very well equipped libraries and so, yes, I don't think there's any excuses really". (lan)

lan is doing something very similar to Huw in positioning the achievement of change as 'totally out of our control'. He locates the source of this as 'down to 
the individual'. He closes off the expected excuse about lack of information resources by noting the availability of 'well equipped libraries'. In doing so lan is suggesting that available knowledge is there for anyone who is suitably motivated. He is perhaps neglecting to acknowledge however that implicit here is that individuals should somehow find the time to do this themselves, outside of their work commitments which presumably don't include academic learning time. So this emphasis on the responsibilities of the individual is to some extent unilateral, it places the full responsibility on staff who are positioned as recalcitrant without acknowledging the significant personal and unpaid resource commitment that may be involved. In a sense it locates blame as well as responsibility with the individual and ignores structural issues that may be at play in these situations.

This category of individual attitude advances an important aspect in that individual attitude was seen as something 'out of their control' which went against sustaining EBPs at their organisation. Huw's quote reflects the cultural aspect of innovation and individual personalities who might be resistant to change.

Ian on the other hand advances that it's up to the individual to keep up to date with evidence. Both of the quotes show an expectation that is being placed on the motivation and commitment of individuals to deliver on the outcomes of EIP services but this can have a slightly evangelical air to it as if there should be no way of criticising or otherwise objecting to the approach. Anderson and West (1998) denote that openness to change is seen as an important component of workplace climate that can impact on innovation in mental health services.

\subsection{Category 2: Protection of Early Intervention in Psychosis Services}

In this category I have collected together data and analysis where practitioners provide justification in an attempt to defend and protect services from external decisions to reduce resources. 
The category outcomes refer to information which is collected and presents the reasoning for early interventions in psychosis services to be funded at an organisational NHS level.

\subsubsection{Outcomes}

The ultimate purpose of EIP services is to improve the outcomes of people and families accessing the service. This however can be challenging in that EIP teams are faced with providing audit data in terms of providing evidence of outcomes. Recording clinical data and inputting data along with writing up notes and updating care plans and having to do clinical work presents day to day challenges for EIP teams (Gillam, 2010). The outcomes category describes how participants saw quality data and key performance indicators that EIP teams should provide almost as justification for the service. Early intervention in psychosis services need to make sure that they audit their clinical outcomes, include quality of life scores. It's much more difficult to cut or curtail a service which is popular with parents or carers (Tait, Ryles \& Sidwell, 2010). In so doing so if services can show good outcomes then it makes it politically difficult to limit these services.

\section{'Communicating that data in a way which justifies the service and also hits certain targets in relation to funding': information is power}

The data extracts below are taken from research interviews with participants Martin, Huw and Steve. The participants construct how important performance and data capture are for an EIP service to survive. In Martin's data below he is cautious in his claim in relation to performance and quality data in relation to key performance indicators. The Quality standard from the National Institute for Health and Care Excellence (NICE) published in 2015 recommended that, "adults with a first episode of psychosis start treatment in early intervention in psychosis services within two weeks of referral' (National Institute for Health and Care Excellence, 2015). 
"I don't know if it's - this is only my perception, but I think particularly our team, has excellent kind of performance and quality data. We are sort of consistently probably the best-performing team in the trust, in terms of meeting the key performance indicators. We never sort of go into the amber or red: this kind of thing". (Martin)

Martin is hesitant in his claims, 'we are sort of' 'probably', and 'it's only my perception'. Martin is also telling us something about EIP teams that they demonstrate a focus and a concern with showing their value to the wider NHS. I read this as awareness of the competitive climate for resources within the wider NHS and EIP services seeing themselves as the 'new kid on the block' and needing to fight their corner for limited funding.

Huw describes how their EIP service had become better at measuring outcomes. He refers to the financial climate and how measuring the performance of the service and how this is communicated in a way which justifies the service and targets. He positions the 'communicating that data' as a facilitator for justification of EIP service and his view that information is power. Huw advances a claim that working with service users in partnership will be more beneficial to them which will be more realistic. Huw denotes 'to use that data in a way which empowers' suggests a nuance of co-production with service users which in turn can facilitate services by helping organisations to become agents of change rather than being service providers.

"given increasing challenges in the financial climate, the service has become better at measuring the outcomes, and measuring the performance of the service, and in communicating that data in a way which justifies the service and also hits certain targets in relation to funding. I think that if we're measuring client outcomes - working with people and collecting outcomes from them - is to use that data in a way which empowers both the client and the clinician so that it's used to support and develop quality, not as an indication of the performance of that staff member, because I think we'd get better quality and more realistic data when it's seen as a part of everyday practice that helps care, not to fit into a more target-based culture of performance. We'd get more data and better data. All of those things, I think, together would evidence and help sustain the quality of what we deliver". (Huw) 
Huw is showing in this account that services are acutely aware of the financial situation within the NHS and the need to proactively collect and demonstrate effectiveness. He seems to be suggesting the services should be recruiting service users in this quest for collecting data as it is something that will be in everyone's best interest longer term. His preferred strategy appears to be to routinize the collection of outcome data and he positions this as a means to improve care as opposed to 'a more target-based culture of performance'.

The quote below is from Steve who is a clinical nurse that specialises in EIP in Wales. In Steve's team they do not have an early intervention in psychosis service. Steve's role is protected or 'ring fenced' to deliver interventions. His team tried to lobby for a hub and spoke model for early intervention in psychosis, but service changes were not made. In Steve's quote below he states that a database was set up to try and capture information for First Episode Psychosis.

"In 2010, I think it was, we set up a database to try and capture information about first episode psychosis, our clients with first episode psychosis coming in to both the CMHTs, well coming into the service, so CMHTs, CAMHS and inpatient services". (Steve)

Steve advances justification for early intervention in psychosis service when he makes reference to 'we set up a database'. Information is power in collating in terms of numbers of people coming through with first episode psychosis. This can be read as some sort of lobbying for an early intervention in psychosis service. Of course this can be naive to think that numbers can justify the need for an EIP service when service changes were not made as will be made clear below in the Wales Annual Operating Framework.

In Wales, the Annual Operating Framework (AOF) (2009 / 2010) which is aimed at securing continuous improvement in the services that are delivered by the NHS to the people of Wales. In the AOF (2009 / 2010) a requirement to develop Early Intervention in Psychosis (EIP) services was a priority, but this was not systematically advanced across all Health Boards in Wales. Early Intervention in Psychosis (EIP) services remain under developed across Wales with inequity 
in the availability, accessibility and provision of high quality evidence based care for young people developing psychotic disorders. To reinforce the national priority for the development of effective EIP services, the Welsh Government established an expert clinical group with service user and carer input to develop an intelligent target for First Episode Psychosis (FEP). There is differing spread and range in early intervention services across both countries. This is illustrated in the quote below from the National Audit Office Wales below.

\begin{abstract}
"Very few areas have a dedicated early intervention (meaning EIP) and treatment service or team. A number of CMHT's report that they include early intervention as part of their remit, and in one area a limited number of CMHT staff have been identified and trained to provide an early intervention service". (Annual Operating Framework, 2010 1.39, p25).
\end{abstract}

The participants have advanced that outcome data were facilitators or almost justification for EIP teams to continue and with their projected survival. When investing in such services such as EIP policy makers are unlikely to invest in such services without seeing such evidence on the effectiveness of the service. I think that in advancing the case for EIP services such services will be strengthened by making the use of follow up data on clients over long term impacts.

\title{
5.12 Summary for Theme Two
}

My analysis showed that fidelity was a barrier for participants. Participants constructed fidelity in different ways such as caseloads, real world clinical practice requiring deviation from models in that service users do not fit the prescribed manual. Dark, Whiteford, \& Ashkanasy, et al., (2015) tell us that the environment (context) in which services are delivered can be aided by the understanding of not only the skills within mental health services but the attitudes of staff towards early intervention in psychosis programmes. The context in which evidence based practices are implemented as clinical reality seems far from that set out in intervention manuals. 
Funding was seen as a barrier when faced with implementing evidence based practices as early intervention in psychosis services are not protected. Funding provision of early intervention in psychosis varies as evidenced in Gareth and Robs extracts in relation to staffing issues. Even where the availability of services is good, the economic climate is placing substantial strains on services which were evident in participants accounts. Practice proficiency included staffing, supervision and training were seen as integral to sustaining an evidence based practice.

Individual attitude was a barrier to adopting an evidence based practice and is also an important contributor to organisation culture. Attitudes can have a negative effect on staff morale and quality of care as demonstrated in Huw's extract. Participants were attempting to provide justifications of early intervention in psychosis services in relation to external decisions to reduce resources. Participants viewed outcome data and how in doing so made it difficult to politically limit these services.

\subsection{Summary of Key Findings from Data Analysis}

Two main themes were produced from the qualitative data. The first theme was important factors in sustaining early intervention teams within participant's organisations. Theme one had three categories which were 'what constitutes EBPs in relation to early intervention'. The claims presented by participants tell us that knowledge means different thing to different practitioners working in different contexts. There is an issue in relation to how to define EBP and according to whom. Evidence based practice is complex particularly in light of patient values, experiences and preferences. Secondly, 'specialised practice' is seen as an important part of the theme because participants argue or make claims on its behalf. Practitioners were advancing the specialist nature of the work they do and that it requires specific awareness of the evidence base to enable decision makers and others to fully understand these services.

The 'champion' category had a sub category of 'senior management'. In order to sustain early intervention teams participants described that there is a need for commitment by certain groups and individuals as champions of the service. 
The subcategory senior management is connected to the category of champions because senior management can initiate change. The hybrid manager (clinician with senior management responsibility) were seen as initiating change at more upper management level due to their knowledge of early intervention and were able to make a good case for early intervention to decision makers and commissioners.

Theme two in which participants constructed their versions of challenges and opportunities to implementing evidence based practice in their services. The theme included two categories of 'barriers' and 'protection of early intervention services'. The barriers included four sub-categories which were (1) fidelity (2) funding (3) practice proficiency and (4) individual attitude. The second category of protection of early intervention services includes one sub-category which is outcome data. Fidelity was a barrier for participants such as caseloads, real world clinical practice requiring deviation from models in that service users do not fit the prescribed manual. Funding was seen as a barrier when faced with implementing evidence based practices. Participants were constructing how funding and support was seen as barriers to implementing EBPs.

Practice proficiency was seen as an integral part of sustainment which included staffing, supervision and training. Individual attitude was seen as a barrier to adopting evidence based practice. Participants were attempting to provide justifications of early intervention services in relation to external decisions to reduce resources.

\subsection{Conclusion}

In this chapter I have shown that my results provide important insights into the nature of issues which interfere with implementation of evidence based practices such as fidelity, funding, practice proficiency, attitudes and outcome data. 
In looking at change interventions in mental health care clinics Kauth, Sullivan, Cully and Blevins (2011) stipulate that the target of change needs to be identified to implement interventions and theories. If the target of change is practitioners then interventions should draw upon cognitive, motivational, and the theory of planned behaviour (Grol, Wensing, Hulscher \& Eccles, 2013). If the social context or network is the target, interventions may come from social learning theory, social network theories and theories on leadership which employ opinion leaders to influence clinicians and engage administrators in promoting change. If the target is the organisation or system, intervention grounded in theories of innovative organisations, quality management, complexity and economics may for instance, encourage creative, decentralised problem solving by front line staff and modify incentive schedules (Kauth et al., 2011).

In the next chapter I will discuss the quantitative results, the qualitative findings and show integration of both sets of findings. I will also show how my research findings address the research questions. 


\section{CHAPTER 6: Discussion and Integration of Findings}

\subsection{Introduction}

The overall purpose of this thesis has been to assess the implementation of evidence based practices (EBP) in mental health with early intervention practitioners. This chapter will explore firstly the quantitative findings, secondly the qualitative findings and thirdly the integration of both methods by using the mixed methods matrix proposed by O'Cathain et al., (2010). I will argue that this thesis has offered insights into the implementation and sustainability of EBPs in early intervention teams in England and Wales. In this chapter I will also show how the findings from each stage of my research addresses the research questions for this study. The research questions for this study are;

1. What are the attitudes of early intervention lead practitioners in relation to evidence based practice?

The EBPAS survey directly answers question one.

2. To what extent is service context and culture relevant to practitioner's experiences of adopting evidence based practice?

The integration of both quantitative and qualitative addresses question two.

3. What do early intervention lead practitioners say are the crucial factors in sustaining or not sustaining practices in their teams in England and Wales?

The qualitative component theme 1 (sustaining EBPs) addresses question three.

4. What do early intervention lead practitioners say are the facilitators and barriers to implementation of evidence based practices in their services? The qualitative component theme 2 (challenges and opportunities) addresses question four. 


\subsection{Quantitative Findings}

The survey component of the study aimed to assess attitudes to EBPs in early intervention practitioners in England and Wales. The quantitative findings from the survey of EIP practitioners in England and Wales provided in Chapter 4 addressed research question one, 'what are the attitudes of early intervention lead practitioners in relation to evidence based practice?'

I have shown that attitudes towards adoption of EBP can be assessed within early intervention teams. The four EBPAS scales represented distinct constructs of: willingness to adopt an EBP given their intuitive appeal, willingness to adopt a new practice if required, openness towards innovation or practice and perceived divergence of usual practice with academic or research based treatments (Aarons, 2005). Attitudes to towards innovation can be a facilitating or restrictive factor in dissemination and implementation of new technologies (Damapour, 1991; Frambach \& Schllewaert, 2002). Attitudes can be a forerunner to the decision of whether or not to try a new practice, and the expressed emotion component of attitudes can impact decision processes regarding innovation (Frambach, 2002, Candel \& Pennings, 1999 \& Rogers, 1995). Attitudes towards adoption of an EBP are proposed to be influenced by organisational facilitators, individual provider characteristics, provider dispositional innovativeness and social networks (Aarons, 2005).

In the next section I will deal with each of the quantitative findings in turn and discuss them with reference to the wider literature. The regression analyses were conducted in order to examine the association of EBPAS subscales (requirements and openness) and the total scores with the demographics and organisational variables. Upon inspection there were strong correlations between the EBPAS requirements and openness scales. It has been previously shown that scores on these subscales are significant positive predictors of attitudes towards the use of psychological interventions and in the current study it appears that where these subscale scores are high then EBP is likely to be easily adopted. 


\subsubsection{EBPAS Requirement Scale: Older Respondents vs. Younger Respondents}

I have shown in Chapter 4 that older respondents to the survey were less likely than the younger respondents to adopt a therapy or intervention if they received training or if it was required by their supervisor, organisation or country.

A reason for this might be that the older respondents were more likely to rely on their experience rather than follow a manual. The older respondents might also be more flexible with service users. The younger respondents were more likely to adopt a therapy or intervention in comparison to the older respondents. In Cunningham's (2013) thesis she applied Aarons' (2004) EBPAS measure to a sample of trainee clinical psychologists. The study findings showed that the openness and requirements subscales were significant positive predictors of attitudes to family intervention. Cunningham (2013) suggests that the requirements subscale was a strong predictor of attitudes, and that this might be that the trainee psychologists are more prominent in a training population. In my findings the younger respondents were more likely to adopt an EBP if required which suggest that they are more exposed to training and transitioning into professional roles which suggests that they are more flexible in regard to learning new interventions.

I conclude from these results of my study that openness and requirements scales were strong predictors of attitudes only for certain professional groups, for example psychiatrists (low score, less open to innovation) and nurses (high score, more open to innovation).

Research by Day, Arthur \& Gettman (2001) and Rentsch \& Kilmoski (2001) found that workers that have pre-professional status are more inclined to the acquisition of new practices because of more flexible knowledge structures. They suggest that such flexibility may facilitate the effectiveness of training in EBPs. I am suggesting that it may be the case that the younger respondents are more flexible to knowledge and hence are more likely to adopt an EBP than the older respondents and this is because of the pre-professional status of the younger respondents. Aarons (2004) says there is evidence that those still 
completing their education (transitioning into professional roles) maybe more flexible in regard of learning new interventions because they are less influenced by a long history of practice. In his study Aarons (2004) found that interns showed more positive attitudes towards new treatments. In my study when I compared professional groups I was able to show that the psychiatrists were less open to innovation than the nurses. Psychiatrists holding doctoral degrees, compared to those who did not hold doctoral degrees, had more reserved attitudes towards EBPs. Aarons (2004), on the other hand, reports that participants with higher educational attainment showed more positive attitudes towards treatments, however, only for intuitively appealing ones. In relation to the differences between my study and Aarons (2004), I conclude that the differences between groups are small, and because my sample is relatively small I suggest that my results should be treated with some caution.

The requirements to provide services in a specified way based on organisational policies or funding exigencies may or may not be followed by service providers (Aarons, 2004). Those participants whose organisations adhered to policies such as the National Institute for Health and Care Research, Policy Implementation Guidelines (DoH, 2001) and the Welsh Psychological Therapies (PIG Wales, 2012) scored significantly higher than those participants whose organisations did not adhere. Garland (2003) notes that there is variability in the degree to which providers adopt and comply with new practices even when required by supervisors or under authoritative orders.

Glisson (2002) tells us that some providers may be more or less compliant to required changes. Individual and organisational variability can affect the degree to which innovations are adopted and sustained in practice. Compliance with requirements differs from openness (McCrae \& Costa, 2003) in that it indicates how employees respond to organisational rules and regulations. An employee may score highly on the characteristic of openness, but may resist authority. 


\subsubsection{EBPAS Openness: Psychiatrists and Nurses}

The psychiatrists were less likely than the nurses to try new interventions. The openness scale assessed the extent to which the provider is generally open to trying new interventions and would be willing to try or use EBPs.

There is considerable supporting evidence of the continued dominance of the medical profession within the clinical setting, particularly in the process adopted to draw up clinical protocols and guidelines. These processes are almost universally led by doctors and, in some settings, all decisions are taken by doctors even without consultation with other professionals. There is also clear structural reinforcement of the dominant medical position, in that many managerial and leadership roles are held by doctors (Dopson \& Fitzgerald, 2009).

Dopson and Fitzgerald (2009) suggest that, in addition to social boundaries, the diffusion of innovations is impeded by the presence of cognitive or epistemological boundaries, such as those advanced by doctors, physiotherapists, clinical professions and managers. Dopson and Fitzgerald (2009) also state that knowledge may diffuse within communities of practice, but stick where practice is not shared. Brown and Duguid (2001a) debate how knowledge manifests 'stickiness' and 'leakiness' concurrently. Professions display different research cultures, agendas, and questions. The barriers have cognitive boundaries as well as a social or an identity based element. These cognitive boundaries operate in combination with the social group boundaries to form the complex social context that influences the career of evidence based health care innovations. This knowledge suggested by Brown and Duguid relates to my findings as it might suggest that certain professions hold onto their knowledge while others share knowledge.

There is an array of reasons in regard to professional discipline and openness to try new interventions which will be discussed below. Openness to change has been identified as an important component of workplace climate that can impact innovation in mental health services (Anderson \& West, 1998). In relation to the organisational structure, organisations with high levels of 
bureaucracy may be less flexible in responding to change and promoting internal change (Frambach \& Schillewaert, 2002). The level of bureaucracy might be an important factor to consider why certain professions are open or not to try new interventions.

In relation to my findings the psychiatrists were less likely to try interventions than the nurses. This might suggest that there are issues with organisational structure (i.e. levels of bureaucracy). Aarons (2004) found that level of bureaucracy was also associated with attitudes towards adoption of EBPs. He found that providers working in low bureaucracy programs were more predisposed to adoption of EBPs scoring higher on the openness and requirement scales. This is an indication of openness to new practices and willingness to engage in new practices when required to do so.

Aarons (2004) tells us that openness to change is also considered an important characteristic of staff learning organisations. The nurses may have more positive working cultures than the psychiatrists. This in turn will lead to more positive views around EBPs. Aarons (2004) found no difference in EBPAS scores by discipline (social work, psychology), but a later study found that providers with a degree in social work had higher openness subscale scores and higher total EBPAS scores than those with a degree in psychology. Clinicians in mental health services with proficient organisational cultures have more positive views of EBPs and are more likely to adopt EBPs (Glisson \& Schoenwald, 2008).

Gallo and Barlow (2012) have reviewed reasons which have been put forward for non-adoption of EBPs by providers. These reasons are: inability to remain current on new developments, perceived difficulties accessing training, selective adoption of EBP and providers' preconceptions and misconceptions of EBPs. Gallo and Barlow (2012) say that clinicians are willing to adopt some EBPs but not others. The literature around the adoption of EBP is seen as an all or nothing phenomenon. One reason may be the level of comfort and familiarity with various interventions, which would most likely predict adoption of any probable new treatments. McGovern et al. (2004) found that clinicians reported 
higher levels of motivation to adopt certain EBPs over others. Clinicians will often encounter acceptance of EBP as a principle or practice ideal, but with minimal commitment to practice change. In a survey of 649 occupational therapists in Australia, 885 agree strongly agreed that EBP improved client care (Bennett et al., 2003). However, 39\% had rarely or not at all relied on current research evidence in their practice over the previous two months. In relation to my own findings the openness domain results suggests an important aspect for future research. Clinicians being exposed to training courses could be one factor in whether the clinician adopts an EBP or not.

\subsubsection{EBPAS Score with Primary Discipline}

The EBPAS attitudinal score results indicate that the psychologists had a significantly lower mean EBPAS score than the nurses. The nurses had a higher EBPAS score (a high score denotes higher attitude to an EBP and a low score is a lower attitude towards an EBP). This might suggest a negative organisational culture within which psychologists work compared to the nurses. Carmazzi and Aarons (2003) found that a negative organisational culture was associated with providers' negative attitudes towards adoption of EBPs while a positive culture was associated with openness to adoption of an EBP. In a qualitative study, Court et al. (2017) examined clinical psychologists' beliefs in relation to NICE guidelines. Opinion was divided within the profession. Clinical psychologists recognized that NICE guidelines have to be seen in the context of the current climate of limited resources. Guidelines were portrayed as being useful to evidence base and valued but there was worry amongst clinical psychologists that the guidelines created a false impression of neatness which is unhelpful in the context of the complexity that every day clinical practice brings in the NHS.

As well as scoring lower on the openness scale, the psychiatrists also had a lower EBPAS attitudinal score which means they had an overall lower attitudinal score to adopting an EBP. In finding the most parsimonious model for the requirements scale, those respondents who were a doctor or fellow were more likely to score significantly more highly on the requirements scale than those 
respondents who were not a doctor or fellow. This finding resonates with Aarons (2004) in educational attainment being associated with positive attitudes to adopting EBPs given their intuitive appeal. Educational attainment has been found to be positively associated with endorsement of evidence based treatments and adoption of innovation (Loy, 1968 \& Ogborne et al., 1998).

Research on provider decision making suggests that providers do not rely on evidence (Rosen et al., 1995) but are more influenced by such factors as lay practices (Rosen, 2003), power structures, ingrained routines and established resource configurations (Rosenheck, 2001). In Aarons' (2004) study he found no significant differences in attitudes towards adoption of EBPs across disciplines. He suggests that other factors might interact with provider discipline in complex ways.

In a survey by Mullen and Bacon (2004), $86.7 \%$ of psychiatrists and $81.4 \%$ of social workers said they were inclined to use guidelines, compared to $54.5 \%$ of psychologists. Addis and Krasnow (2000) tell us that provider receptivity to empirically based treatments varies considerably within professional disciplines but not between disciplines. Aarons (2004) surveyed clinical and case management service providers from 51 public sector programmes providing mental health services to children, adolescents and families and found that interns endorsed more positive attitudes (than staff) towards the adoption of EBPs. There were no significant differences found in attitudes towards adoption of EBPs across disciplines. The literature on diffusion of innovation makes the distinction between various types of decision to adopt or reject an innovation such as EBP. The decision may be that it is made by an individual independently of decisions made by other members of the system; or the decision may be collective, in that all members the system reach a consensus and all units are expected to conform to the decision made. Authority based decisions are made by a small group of individuals who possess power, status, or technical expertise. The role of authority based decisions provides the fastest rate of adoption (Rogers, 1995). 


\subsubsection{Primary Discipline with Service Models}

Two separate regression analysis with the items primary discipline and the type of service model (inpatient) provided showed that the psychiatrists and psychologists were less likely to adopt a therapy or intervention if it was required by their supervisor, organisation or country. The second regression analysis with primary discipline and service model (outpatient) showed that the social workers and the therapists were scoring higher than the other respondents.

In relation to the psychiatrists and psychologists scoring lower in the inpatient service model, Damanpour (1991) says that the type of services to be delivered (e.g. outpatient, inpatient) may be related to adoption of innovation. There is evidence that organisation innovativeness varies by type of organisation. Aarons (2004) found that outpatient providers were more open than those working in case management programmes who were less open to adoption of EBPs suggesting that it is important to consider the programmatic context into which EBPs are disseminated. Future studies could look at how program type interacts with attitudes.

\subsubsection{Interactions}

During the quantitative analyses, I found two statistically significant interactions. The first interaction was age by gender and the second was age by policy. The interpretation of the age by gender interaction is as follows.

Among the younger respondents, female participants scored higher on the EBPAS requirements scale than male participants, whereas in the older age group, the males are outperforming the females.

There was an interaction with Policy Implementation Guideline (PIG) in relation to the younger and older respondents. The younger respondents that did not adhere to the PIG scored lower than those younger respondents who did adhere to the PIG. The older respondents that did not adhere to PIG scored higher than the older respondents who do adhere to PIG. 
In the literature around the EBPAS requirements scale, Garland, Kruse and Aarons (2003) tell us that requirements to provide services in a specific way based on organisational policies or funding exigencies may or may not be followed by service providers. For example there is changeability in the degree to which providers adopt and comply with new practices even when required by supervisors or organisational policies. Glisson (2002) proposes that some providers may be more or less compliant with required changes, individual and organisational variability which can affect the degree to which innovations are adopted and sustained in practice. In relation to my findings with the older and younger respondents adhering to the policy or not this might resonate to the degree in which organisational variability permeates an innovation and whether a practice is sustained or not.

\subsection{Qualitative Findings Discussion}

The qualitative component of the study involved eighteen semi-structured interviews with early intervention practitioners. Two themes were derived from the interviews which were labelled as 'sustaining evidence based practices' and 'challenges and opportunities'. The themes were constructed by constitutive categories and below I discuss the main findings arising from my analysis of these data. The research questions being addressed by these findings are; What do early intervention lead practitioners say are the crucial factors in sustaining or not sustaining practices in their teams in England and Wales? What do early intervention lead practitioners say are the facilitators and barriers to implementation of evidence based practices in their services?

\subsection{Sustaining Evidence Based Practices: Evidence and Context}

In the first theme my analysis examined how participants constructed the important factors in sustaining early intervention teams within their organisations. An important finding from the research was how evidence based practice was not such a straight forward notion. The role of context is pivotal in conditioning the spread and sustainability of new practices (Strang \& Soule, 1998) and in determining readiness for change in adopting health services 
innovations (Greenhalgh et al., 2004). For example, participant Phil talked about what constitutes evidence and evidence that comes from NICE is not always implemented or delivered as per se. Phil argues that EBP is not so clear cut initially as perhaps he had initially thought. He also disputed the primacy of guidelines and argued that NICE do not always capture relevant evidence and may prioritise some types of knowledge over others. Proctor et al., (2011) says established evidence for a proven treatment does not ensure successful implementation. Implementation also requires addressing a number of important contextual factors, such as provider attitudes, professional behaviour, and the service system.

Dopson et al., (2002) describe how context is an important mediator of diffusion of innovations which they say is a poorly understood mediator. Dopson et al., argue that organisational context is a sophisticated notion which acknowledges local contexts are multidimensional, multifaceted configuration of forces, which can be seen as a set of stationary independent variables or an ordered series of hierarchical layers, but as a syndrome of forces, which interact in complex ways.

\subsubsection{Knowledge}

In theme one, category one, participants described evidence based practice in relation to early intervention. In the data extract from Phil's research interview he talked about what constituted early intervention and that evidence that comes from NICE is not always implemented. There are two issues for discussion here; there is clearly some dispute and the idea that NICE doesn't have a monopoly on what constitutes evidence is open to contest and implementing evidence based practice is much more complex than a seemingly straightforward route of passing on new knowledge to practitioners. These will be discussed below.

In terms of looking at knowledge Crilly et al., (2012) say that epistemology matters in that epistemologies demand different responses to create and exploit knowledge for example knowledge-as-practice, knowledge-as-data and 
knowledge-as-meaning. The breadth of what is considered evidence is therefore wide and dynamic (Shaxson, 2005). Rycroft-Malone et al., (2003) say that there is no such thing as 'the' evidence. In a study by Dopson et al., (2002) who conducted a comparison and synthesis of seven evidence based practice studies which included 49 cases with 1400 interviews. One of the themes from their analysis was that they suggested there were precise clinical topics supposedly capable of scientific testing and proof.

Guidelines alone are not enough to implement evidence based practices, systems change is required (Dopson \& Fitzgerald, 2005). Despite the assorted definition of evidence, it would be a mistake to assume that in reality all forms of evidence share equal importance, relevance or weighting.

Nutely (2003) highlights the fact that the interaction between policy makers and researchers is limited by the divergence of these two worlds. They use difference languages, have different priorities, different agendas, different timescales and different rewards systems. Sutcliffe and Court (2005) say that there needs to be an increased communication interaction between research and policy worlds such as through discussion and joint training. In terms of the policy world there are factors that impinge on this. These factors occur at an individual level for example policy maker's own experience, expertise and judgement. At an institutional level, there might be constraints, which will limit the extent to which evidence can affect policy and pressures to process information quickly. Lloyd and King (2009) say that EBP is not a one size fits all approach to treatment. Nor is it a paternalistic application of the principle that the practitioner knows what is best for the client. However they say it implies careful assessment of client goals and priorities and sensitivity to cultural background and values as they relate to treatment. This provides a collaborative process by which the practitioner and client make decisions about treatment in the light of goals and preferences. 


\subsubsection{Shared Understanding}

In relation to theme one category 2 of specialised practice participants were describing a lack of understanding that make decisions on behalf of the trust this is evident in Jeremy's extract below.

In the data extract from Jeremy's research interview he makes a confident claim that people making decisions are not aware of actual service delivery. There is a contentious issue raised here around relationships between clinicians and general managers. In research by Dopson and Fitzgerald (2005) they showed that there was a lack of managerial engagement in some of the clinical effectiveness and evidence based health care projects. On the other hand, they found that many general managers that they interviewed had been well informed and positive about local initiatives which suggest that projects which had made most progress were more likely to have had active senior management support. They also found that general managers and clinical hybrid managers often express strong commitment to the principle of evidence based practice and a belief that it is important for quality of care. A lack of involvement may stem more from a mixture of lack of appropriate knowledge and lack of time, given the pressure to meet other performance management requirements rather than from lack of interest.

Defining the relative roles and responsibilities of clinical managers and general managers for decisions about the adoption of innovations is crucial. Furthermore, the balance of power between clinical hybrid managers and general managers is also likely to have a major influence on decisions, especially at a local level. The differing orientation and training of clinical professionals compared with hybrid managers means that they will probably weigh the advantages and disadvantages of an innovation according to varied criteria. 


\subsubsection{Transformational Leaders: Champion Leads on Senior Management}

In theme one, category three, participants described how the presence of champions was important in sustaining evidence based practices and how they acted as agents for change. Participant Steve referred to individual qualities such as 'enthusiasm' and 'optimism' which are individual qualities that he suggested that his colleague had for maintaining interest and pursuit of early intervention. The presence of a champion was also found in Swain et al., (2010) which was seen as an important aspect of the initial implementation phase, and it was also important in the sustaining phase. I am advancing the claim here that 'qualities' that Steve refers to are a transformational type of leadership which is an important aspect of context. Opinion leaders who are local, on hand to promote the work and begin the process of changing service culture have shown to be successful, by influencing dissemination from the bottom-up (Dopson, FitzGerald \& Ferlie, et al., 2002).

Avolio and Bass (1999) describe how transformational leadership which is motivational, and can engage staff in supporting the vision of a leader and promote climate for innovation and change. Transactional leadership style is suitable in many settings and may support adherence to practice protocols but not necessarily openness to innovation. A transformational leader would influence attitudes by inspiring acceptance of innovation through the development of enthusiasm trust and openness (Aarons, 2006).

The presence of champions is consistent with EBP implementation conceptual models such as the Exploration, Preparation, Implementation and Sustainment (EPIS), (Aarons, 2011). Sustainment of practices are more successful when placed in an organisational culture that highly values EBP use and facilitates team participation (Aarons et al., 2016). 


\subsubsection{Senior Management}

Within category three which I labelled champions, a subcategory called senior management branched from champions. Like the champion category, senior management can influence change and act as an agent and makes a case for investing in early intervention. Participants made reference to a clinical director who was also a clinician working with an early intervention team.

An important aspect of senior management sub category was the clinical director who operates at two levels. The first is management level and the second is the clinical expertise that one can bring to this role. This is seen as the 'two-way window' because clinical directors exhibit Janusian thinking which is the ability to join two sets of traditionally contrasting ideas (McCasky, 1998).

Champions, who have earned and maintained and who are often at the centre of interpersonal communication networks (Rogers, 1995), can be effective messengers for EBP. Champions and senior management possess leadership qualities. Research has identified two types of leadership (Avolio, Bass, \& Jung, 1999) which are effective for services team: transformational and transactional. The transformational leadership model has been elaborated by Bass (1985). Leaders who use transformational skills encourage team members to view their work with work related problems. Transactional leadership is more focused on the day-to-day tasks which need to be completed to keep a team or a department running smoothly (Garman, Davis-Lenane, \& Corrigan, 2003). Garman et al., (2003) says that part of this process involves using contingent reward behaviours where team members are rewarded by the leader for achieving established goals or tasks. Transactional leadership is theorised to involve management by exception behaviours (Deane \& Gourney, 2009).

In principal, champions could provide effective clinical leadership for organisational change. Dopson and Fitzgerald (2009) found two major categories of opinion leaders: experts and peers. Expert opinion leaders are seen as the higher authority, who are able to explain the evidence and respond to academic debate. They may be important in the early stages of negotiating the evidence. Peer opinion leaders, on the other hand are individuals who have 
applied the innovation in their own practice and can give colleagues confidence and support. Dopson and Fitzgerald (2009) concluded from their study that clinical opinion leaders needed to have and retain genuine credibility with their colleagues to be effective. Dopson and Mark (2003) provide an overview of issues in leadership research in health care organisations. They say that leadership is more likely to be effective when it's distributed throughout the organisation and issues of professional power are understood.

Long (2013) states that a challenge to translational research effectiveness is the mediating role of cultural factors. Centrally located leaders who have relevant expertise and a vision of how successful translational research is achieved (Grey, 2008) or who can act as influential opinion leaders for the adoption of new findings, or who use their positions as brokers between two cultures are well placed to mediate and smooth members interactions (Skokols, Misra, Moser, Hall, Taylor, 2008).

Aarons (2006) suggested that transformational leadership would influence attitudes by inspiring acceptance of innovation through the development of enthusiasm, trust, and openness, whereas transactional leadership would lead to acceptance of innovation through reinforcement and reward. Leadership therefore is important to consider in relation to acceptance of innovations and to work attitudes, perceptions, behaviour. Leadership is associated with organisational and staff performance. Hence, I propose that leadership is likely to influence attitudes towards adoption of innovation of evidence based practices.

Research into organisational change and diffusion has emphasised the fundamental role of context in the sustainability of new practices (Strang \& Soule, 1998) and in determining the readiness for change in adopting health service innovations in particular (Greenhalgh et al., 2004). Implementing evidence based practice requires whole system change implicating the individual and the organisational levels (Grimshaw, Eccles \& Tetroe, 2004 \& Dopson \& Fitzgerald, 2005). The literature on achieving change emphasises the 
importance of considering context and choosing change interventions that suit that context.

Evidence based practices implementation has to attract resources, time and sustained attention if it is to succeed. At the corporate level health care organisations can adopt evidence based practice as a formal policy, it only becomes real if it is enacted by a large number of front-line clinical groups over which senior management has limited control.

\subsection{Challenges and Opportunities}

The challenges and opportunities theme helped me address research question two 'to what extent is service context and culture relevant to practitioner's experiences of adopting evidence based practice?' whereby I combined the quantitative and qualitative components.

The challenges and opportunities theme two, category one 'barriers' helped me address research question four, 'what do early intervention lead practitioners say are the facilitators and barriers to implementation of evidence based practices in their services?'

Participants identified barriers or obstacles to adopting evidence based practices at their organisation these were fidelity, funding, practice proficiency and individual attitude. To bridge the gap between scientific evidence and patient care Grol and Wensing (2004) highlight the need for an in-depth understanding of the barriers and incentives to achieving change in practice. Participants identified the need to protect early intervention services. The category 'outcome' refers to information which is collected and presents reasoning for early intervention services to be funded.

\subsubsection{Fidelity}

Torrey et al., (2001) say that evidence based practices are complex and mutlifaceted interventions that are difficult to implement without pre-existing support and structure. Davies et al., (2000) talks about how organisational structures and cultural norms that prevail within an organisation can enable or disable particular forms of individual practice. 
Fidelity was seen as a barrier in implementing evidence based practices as the service user does not fit with the prescribed manual. In a study by Botvin, (2004); Hill, Maucione and Hood, (2007) they identified several barriers to maintaining implementation fidelity in real life context. These included local adaptations of interventions, individual variations in practitioner adherence and competence, lack of available training and technical support, limited resources for supporting the intervention at the site level and competing demands for practitioners time which diminishes their commitment effectiveness. Clinical guidance has been created to try and reduce this inequality (National Institute for Clinical Excellence, 2014) including specific recommendations concerning psychological therapies. Cognitive behavioural therapy (CBT) and Family intervention (FI) have been recommended for the treatment of people with schizophrenia (National Institute for Clinical Excellence, 2014).

In a study by Haddock, Eisner and Boone, et al., (2014) they reported rates of implementation far below $5.3 \%$ for CBT and $1.1 \%$ for family intervention. It appeared that despite the widespread dissemination of guidance, there is still unmet need in relation to access to psychological interventions, and that awareness of them does not necessarily guarantee successful implementation. Berry and Haddock (2008) have shown that barriers to implementation behaviours are met not only at the individual level, but also at the organisational level.

In the current study participants spoke about fidelity in slightly different ways. Participant Anne described EIP model fidelity in relation to adherence indicator of caseloads. Anne advances the claim that caseloads should remain low as anything above 15 people and the model fidelity is lost. In a study by Nelson et al., (2006) practitioners indicated that their heavy caseloads did not allow them the time to learn new approaches and that they did not have the training or supervision needed to implement EBP. The lost generation report by Rethink Mental Health charity survey found that nearly a third of teams (31\%) reported that their caseloads had risen above the recommended level. Services 
stipulated that this was due to staff losses, pressure to meet targets and difficulties in discharging people to other mental health teams.

In the data extract from Phil's research interview he referred to clients not fitting the prescribed manual. Therefore having flexibility to adapt within real world clinical practice is essential. There is an issue in terms of real world clinical research and mental health research and this is echoed by Cleary et al., (2010) highlights that for research findings to make the leap to evidence based practice, the research needs to include real-world consumers and families typical of clinical practice supported by clinically relevant outcomes. Oestrich, Austin \& Tarrier (2007) say that when designing and implementing any research, the influence of patient characteristics, staff variables and organisational context needs to be considered.

\subsubsection{Funding}

Practitioners described how funding was a barrier when faced with implementing evidence based practices. There are likely to be numerous decision points and obstacles when implementing early intervention in psychosis teams. Resources for mental health services are not infinite and generally scarce. When resources are abundant it seems to be common to provide primarily for patients who have established disorders and to continue to configure services to cater for such patients (Edwards \& McGorry, 2002). As McGorry (2000, p.22) states:

'Investment in new and better treatments must continue, but we must learn to deliver them effectively in real-world settings'.

Participant Jeremy talked about how his organisation had a lot of financial pressures put on them as a team to establish an early intervention service. He also asserted the 'established CMHT' had made it more difficult to apply treatment to an early intervention population. Jeremy advanced that the established services are operating like the medical model. The philosophy and evidence for early intervention are not understood. Gareth on the other hand argued that funding was a barrier to sustaining early intervention in terms of 
recruitment and staff shortages for example recruiting doctors. Participant Rob stated that they do not have funding for a fully-fledged early intervention service. They operated a pilot project within the CMHT service. Funding provision varies this is evidenced in Gareth and Rob's quotes.

Analysis by the Health Services Journal shows (2018) that nearly a quarter of clinical commissioning groups are spending less than the estimated sum needed to provide proper care to seriously ill mental health patients. Funding is a real world consideration in which the provision varies.

\subsubsection{Practice Proficiency}

Practice proficiency was viewed by participants as integral to sustaining evidence based practices. Practice proficiency included staffing, supervision and training in which in their view were seen as barriers to adopting evidence based practices. In a study by Pagoto et al., (2007) in a survey of 37 practitioners, lack of time and money for training, a part of their logistical concerns surrounding implementation and issues with obtaining training were identified barriers in their study of early intervention services.

In Swain et al., (2010) supervision was viewed as integral to sustaining a practice, but site-level respondents reported that supervision time decreased after the initial implementation period due to time constraints. There is an emphasis on the need for appropriate facilitation to improve the likelihood of success (Swain et al., 2010). The type of facilitation, and the role, and skill of the facilitator that is required is determined by the "state of preparedness" of an individual or team, in terms of their acceptance and understanding of evidence, the receptivity of their place of work or context in terms of resources, culture and values, leadership, style, and evaluation activity. 


\subsubsection{Individual Attitude}

Individual attitude was seen as a barrier to adopting an evidence based practice. An important aspect of organisational culture is individual attitude as this can impact on how new technologies are considered and adopted in practice. Participants Huw and lan both responded to the question, 'what things either in your control or out of your control that you think worked against sustaining EBPs. Huw talks about the culture change aspect of the organisation and how this was out of his control in implementing EBPs. He refers to the atmosphere and the climate in which people work and there being 'strong personalities' who are not motivated to address those cultural elements. lan on the other hand advances the claim that it's up to the individual to keep up to date with developments in relation to evidence based practices.

lan's quote puts the onus on individuals to find the time to use libraries to do literature searches, he locates blame a well as responsibility with individuals and ignores structural issues that may be at play in these situations. Panzano and Herman (2005) say that in thinking about implementing new practices staff need the opportunity to develop new knowledge and skills. These could be internal (such as work satisfaction, values) and external factors (such as success stories, respect from peers, promotion) to implement the behaviour change. However, the opportunity to implement a particular EBP may require overcoming barriers such as lack of time or resources (Lloyd \& King et al., 2009). This category individual attitude resonates with the practice proficiency category in relation to how important service context and organisational culture are. Supervision and resources promote EBP, but lack of these makes implementation of EBPs difficult.

\subsubsection{Protection of Early Intervention Services}

The protection of early intervention services is an attempt to provide justification to defend and protect services from external decisions to reduce resources. This provides an answer to my fourth research question of 'what do early intervention lead practitioners say are the facilitators to implementation of evidence based practices in their services'? 
Participants Martin, Huw and Steve construct how important data was for early intervention teams to survive. Huw's talks about communicating data as a facilitator for justification of early intervention teams in which he positions information as power. He talks about using data which suggests a degree of coproduction with service users. This in turn facilitates services by helping organisations becoming more innovative whereby service users become the agents of change rather than service providers.

\subsubsection{Summary}

Evidence of what works frequently has a disappointing impact on eventual policy and practice. Traditional conceptions of the role of evidence have placed unreasonable and unhelpful boundaries between the creation and use of research. Evidence based practice has to be viewed as a partnership activity between all the key stakeholders, with no clear discontinuities between evidence creation, validation, dissemination or use. Literatures on diverse learning as well as personal learning, professional decision making, and the dynamics of organisational change and learning can all contribute insight into these partnerships. Davies and Nutley (2000) say what is needed is an approach that combines:

Insights from systems thinking (in terms of the setting the context within which evidence is to be used; Understanding of individual decision making and behaviour change (which acknowledges the importance of craft routines and tacit knowledge held by professionals); Awareness that the nature of the innovation being promulgated will influence its diffusion (and in particular, the fit between innovation the context and the adopters).

Psychological behavioural change such as the Theory of Planned Behaviour (Ajzen, 1991) has been widely used in implementation science to study determinants of clinical behavioural change (Nilsen, 2015).

The Theory of Planned Behaviour alone is not enough to bring change to healthcare settings. A whole systems approach is required which encapsulates, the individual and at an organisation level. 


\subsection{Integration of Quantitative and Qualitative}

This study was an explanatory sequential mixed methods design consisting of two distinct phases: quantitative methods via a survey followed by qualitative methods in the form of research interviews (Creswell et al., 2003). Mixed methods have faced philosophical, methodological and political challenges. Criticisms of mixed methods have come from purists who see them as a threat to their traditional qualitative or quantitative domains. They are concerned that mixed methods might somehow dilute the purity and legitimacy of traditional methodologies (Teddlie \& Tashakkori, 2009).

\subsection{Conceptualising Integration}

Bazeley (2009) tell us that the key to conceptualisation on integrated research is the requirement that each method retains its own character: different data types are not transformed into one type and then analysed using one analytic method. The retention of methodological character allows the findings of each dataset or method to contribute equally to answering research questions in their own paradigmatic terms and the methods interface with each other through some kind of designed and systematic combination.

For the integration of quantitative and qualitative components, I decided to follow O'Cathain et al.'s (2010) paper in which the authors see a role in examining a subset of the data. Hence the subset is for integration purposes only. The previous discussion in this chapter has focused on the whole of the data. A unique aspect of mixed methods studies is the availability of both qualitative and quantitative data on the same cases (O'Cathain et al., 2010). The mixed methods matrix was used to look at the main summary findings from the quantitative component of the survey and the main themes from the qualitative aspect of the study and whether the extent to which the findings from each strand agreed with each other.

In looking at the mixed methods matrix (Appendix 15), the rows represent the participants for which there is both qualitative and quantitative data, the columns display different data collected on each case. O'Cathain et al., (2010) 
tell us that this allows researchers to pay attention to surprises and paradoxes between types of data on a single case and then to look for patterns across all cases in a qualitative cross case analysis.

\subsection{Mixed Methods Matrix (Integration of Quantitative and Qualitative Findings)}

The mixed methods approach was used to examine early intervention practitioners' attitudes towards the implementation of evidence based practices in England and Wales. The integration of findings examined quantitative and qualitative results and I will discuss below where these converged or diverged to indicate integration as suggested by O'Cathain et al., (2010).

Quantitative analysis indicated a variety of positive and negative scores on the requirement scale, openness scale and EBPAS attitude score.

Qualitative interviews revealed two themes. Theme one was important factors sustaining evidence based practices in early intervention teams within practitioner's organisations. The categories within this theme were: what constitutes EBPs in relation to early intervention, specialised practice and champions.

The second theme was challenges and opportunities to adopting evidence based practices that focus on claims relating to barriers and protection of early intervention services such as outcomes which is the justification of resources at an organisational level.

The EBPAS requirements scale assessed the extent to which the provider would adopt an EBP if it were required by organisation, supervisor or country. In looking at the integration of the two data sets as whole there is no dominant pattern in this integration. Quantitative findings about positive EBPAS requirement scores converged with respondents interviews such that respondents discussed and implied a direct link with evidence, policy and how caseloads were important for model fidelity. Where there was an overall positive EBPAS score respondents discussed how internal practice champions in 
support of sustainment (hybrid managers' clinicians in a management role) were important to promote change at upper management levels.

The quantitative findings with positive EBPAS requirement scores diverged from the qualitative findings in that respondents discussed barriers to adopting EBPs such as: individual attitudes such as strong personalities not motivated in changing culture (resistance to change), recruitment issues, staff shortages, supervision and training, fidelity requires time and is a difficult area, lack of understanding about specialist practice, EBP is not a straightforward notion.

\subsubsection{Requirements Scale}

The EBPAS requirements scale assessed the extent to which the provider would adopt an EBP if it were required by organisation, supervisor or country. Aarons (2005) tells us that requirement to change practice varies from person to person. For example, some providers may be more or less compliant with required to changes: individual and organisational changeability can affect the degree to which innovations are adopted and sustained in practice (Glisson, 2002; Rogers, 1995). Participants' responses varied. The older participants had a lower requirement EBPAS score than the younger respondents. In some of the respondents that had a higher score on the requirements scale there was agreement in their extracts in relation to adopting policies.

In the case of respondents who had lower requirement scores there appeared to be congruence between what they said in their research interviews and their scores. There was a common thread of negative requirement for adopting an EBP and lack of understanding in relation to specialised practice. The nurses had positive requirement scores when asked does your organisation adhere to polices such as: PIG, NICE and Welsh psychological therapies. Some psychologists and psychiatrists also had positive scores on PIG, NICE policies whilst other psychiatrists had a negative score on requirements for policies. 


\subsubsection{Openness}

As mentioned above previously, openness to change has been identified as an important component of workplace climate that can impact innovation in mental health service programmes (Anderson \& West, 1998). The openness scores varied between the psychiatrists and nurses. The psychiatrists had a lower openness score than the nurses. The psychiatrists also had a lower overall EBPAS attitudinal score than the nurses who had a higher EBPAS attitudinal score.

The psychiatrists that had a lower EBPAS score also spoke about resistance to change in the interview extract. One nurse who had a positive EBPAS score, but spoke about organisational issues in terms of barriers such as recruitment and staff shortages. The psychologists had low EBPAS score, for example in one respondents extract he spoke about EBP not being a straight forward notion and that NICE don't have the monopoly on what constitutes evidence based practice. One respondent talked about how fidelity required significant amount of time and that practice proficiency in terms of supervision and training were barriers to adoption EBP.

Fidelity is a key ingredient for the implementation of evidence based interventions in community settings (Breitenstein et al., 2010). Glasgow, Lichenstein and Marcus (2003) say that, for large scale dissemination of interventions to be effective, researchers need to understand the processes required in order to implement the intervention consistently, especially when different practitioners with different levels of expertise are implementing the intervention in different contexts. One respondent had a low score on the requirements scale in relation to policies such as PIG, NICE and Welsh psychological therapies and in his interview extract he referred to fidelity and how it is a difficult area.

\subsection{Conclusion}

The process for integrating mixed methods does come with challenges. The first challenge is to identify promising threads. Mixed methods studies tend to be 
successful in health services research in that the qualitative and quantitative components are completed as planned (O'Cathain, 2008). The main issue with mixed methods is the quality issue in terms of lack of transparency of mixed methods with the individual components. O'Cathain (2008) denotes that due to the fact that there is a historical dominance of quantitative methods in health services research the qualitative component are more likely to be poorly described than the quantitative component.

In this chapter I have shown that attitudes are a strong predictor within early intervention teams. My findings have shown differences in professional disciplines in relation to openness and requirements of an EBP. The qualitative findings have provided participants talk of sustaining EBPs and identified barriers to adopting an EBP. This knowledge originating from this research may prove useful for practitioners and organisations alike as they seek to plan and develop mental health services that are informed by evidence of effectiveness. Implementing evidence based practice is more complex and managers need a sophisticated understanding of EBP. Managers can play a vital role in developing EBP but need to be aware of the contact change that presents in real world clinical practice.

The integration of quantitative and qualitative findings enabled me to explore deeper meanings of what practitioners were saying beyond the impact of attitudes as barriers to utilising knowledge and skills. The interviews allowed me to gain deeper description and explanations to be offered that the survey did not but these may also have shown where the limits are to assessing attitudes when other factors are in play e.g. organisational factors. 


\section{CHAPTER 7: Conclusion}

\subsection{Introduction}

In this final chapter I provide a summary of the thesis and a review of the limitations of the study. I show my original contribution of the field of implementation in early intervention services by assessing practitioners' attitudes to adopting an EBP. A reflective commentary is provided to show how the methodological challenges and ethical issues that arose during the course of the research project have been addressed. Finally the implications of the study for implementation policy and practice are considered, and suggestions are made for future research.

\subsection{Original Contribution of this Thesis}

In this thesis I have presented an analysis of data collected to assess attitudes towards the implementation of EBPs in early intervention teams in England and Wales. This is the first study of this type to examine attitudes to evidence based practice in England and Wales. I have shown that attitudes towards adoption of EBP can be assessed within early intervention teams and that the qualities of the EBPAS scale requirements and openness were strong predictors of assessing attitudes in early intervention practitioners. I have shown that the openness and requirements subscales were good predictors of assessing attitudes towards EBP, in particular only for certain professional groups, for example psychiatrists (low score, less open to innovation) and nurses (high score, more open to innovation). The qualitative interviews revealed two themes. Theme one was important factors sustaining evidence based practices in early intervention teams within practitioner's organisations. The categories within this theme were: what constitutes EBPs in relation to early intervention, specialised practice and champions. The second theme was challenges and opportunities to adopting evidence based practices that focus on claims relating to barriers and protection of early intervention services such as outcomes which is the justification of resources at an organisational level. The integration has enabled me to explore deeper meanings of what practitioners were saying beyond the impact of attitudes as barriers to utilising knowledge and skills. 
Sequential qualitative findings provided insights in relation to day-to-day practice issues identified by early intervention practitioners as sustaining EBPs and the challenges and opportunities to adopting EBPs. I have shown via my analysis of research interviews that these practitioners are concerned with sustaining evidence based practices in terms of what constitutes EBP in relation to early intervention, specialised practice, champions and senior management. Early intervention practitioners identified through talk the challenges and opportunities to adopting an EBP such as the barriers to fidelity, funding, practice proficiency, individual attitude, the protection of early interventions services and outcome data. These combined findings suggest that the uptake of evidence based practices is variable among individuals depending on age and experience and contingent on a range of contextual factors that are prone to the vagaries of individual organisation decisions.

Two theories were introduced in the introduction of this thesis. The first was the Theory of Planned Behaviour and the second was Systems Theory. These theories can help guide implementation practice because they potentially can identify barriers and facilitators which might be important when addressing the implementation endeavour. Dijkstra, Wensing, Thomas et al., (2006) says that we must be attuned to such growing awareness of factors relating to the social, organisation and economic contexts which influence the successful uptake of knowledge translation.

\subsection{Limitations of the Study}

In undertaking this project it has been possible to gain insights into services implementing EBPs in mental health within Early Intervention in Psychosis teams. However, as with any research project, this study has certain limitations. The research has revealed a rich picture of early intervention practitioner attitudes and sustainability of EBPs in Early Intervention in Psychosis teams. There was a limitation in relation to the way the data were collected. The quantitative component involved the use of an online survey. As is the case with surveys more generally if respondents had initial queries then I was not available to answer these. To ease the burden on busy practitioners' the survey 
was designed to be completed within ten to fifteen minutes. From my initial estimate of 110 early intervention practitioners, 70 of these practitioners participated in the survey. Although this is a high response rate the numbers recruited may have reduced the range of statistical analysis possible and reduced the opportunity to make broader claims based on this analysis.

Another possible limitation in this study relates to the subscales of the EBPAS. For example, the divergence scale assesses the extent to which the provider perceives evidence based practices as not clinically useful and less important than clinical experience. The divergence scale had poor reliability (alpha .47; Eigen value .785). Aarons (2004) says that the item analyses shows that the reliability coefficient for the divergence scale would not have been improved by removing items from the scale. The internal consistency reliability for the divergence scale was not at its best (<.60), attitudes have been reported as an important construct (Garland et al., 2003) and so the subscale was retained. Aarons, Calfri, Lugo and Sawasky (2012) expanded the domains of attitudes towards evidence-based practice to EBPAS 50. The EBPAS 50-item scale which combines the original 15 items with the new 35 items comprises the EBPAS 50-item version adds to the understanding of provider attitudes towards adopting EBP (Aarons, 2004; Aarons et al., 2007).

The EBPAS 50 study does pose limitations in that no confirmatory analyses were conducted and that the factor structure of the EBPAS 50 requires further validation with all the 50 items.

The method used for the qualitative component was semi-structured interviews, which has limitations. It could be argued that if the researcher concentrates too much on a pre-defined list of topics there is a risk that other information may be missed, for instance missing cues from participants that might have led to more fruitful avenues of discussion. Deviation from the interview schedule to explore certain topics in more detail can mean that some topics are not covered at all in every interview. The method of telephone interview has some limitations as well. Irvive (2011) tells us that telephone interviews tend to be shorter than face to face interviews; research participants tend to speak for a smaller proportion of the time; and participants tend to respond in less detail than in face to face 
interviews. My decision to use telephone interviews was in part related to the time available the diverse geographic spread of the participants. With more time and resources it might have been possible to conduct these interviews person or via real time computer based video technology.

Tashakkori and Teddlie (2003) denote that one limitation of mixed methods is the perceived incompatibility of mixing methods that have two opposing world views, with differing ontologies, epistemologies and conceptions in the pursuit of truth and knowledge in once research project. Rather than trying to apply findings in practice in a useful way mixed methods research generates more epistemological debate and continues to do so. Creswell and Plano Clark (2007) point out that conducting mixed methods research is not easy. In addition, mixed methods research requires that researchers develop a broader set of skills that span both the quantitative and qualitative paradigms (Azorin \& Cameron, 2010).

I acknowledge that I did not consult service users and carers early in the research process. As a University employee with no practitioner background I had no access to patients and I was unable to obtain a patient view on my proposal at that time. I am aware that patient views are very beneficial to contributing to better research questions and getting policy into practice (Simpson, Jones, Barlow \& Cox, 2014).

\subsection{Strengths of the Current Research}

The online survey data collection was a good way to reach the research target population who were dispersed across significant geographical space and would have been difficult to access via other means. The Survey Monkey software that was used was easy to administer and it was very flexible and allowed participants to complete the survey in their own time. Research via the internet is increasingly becoming popular as it is practical for researchers and participants (Kraut, Olson \& Banaji, et al., 2004).

In deciding to use a mixed methods approach I was able to gain a valuable perspective using data from both components of the study. The mixed 
sequential explanatory design, using a quantitative survey followed by qualitative interviews, enabled me to carry out more a detailed investigation of the phenomena being studied than would be gained through the use of just one method of data collection.

The work presented in this thesis shows that there is still a gap between evidence and practice which still exists and is of concern. I have shown that clinicians on the whole are keen to adopt evidence based practices and show a range of complex but rational reasons for not doing so in all circumstances. It is clear then that changing clinician attitudes is not enough on its own; a whole system change is required. My analysis also suggests that accounting for the practicing clinician's perception of the evidence and their experiences with that evidence should be addressed when considering how evidence is used in practice.

\subsection{Implications for Policy and Practice}

In this section I discuss implications for reflective practitioners in relation to what health care policy makers and practitioners can usefully learn from the findings of this thesis for their practice.

There is a broad consensus that a number of barriers stand in the way of implementing systemic change in mental health services. The most commonly imminent barrier is funding, which forms a core element of debate on achieving parity of outcomes. Community services have undergone considerable reconfiguration in recent years including remodelling, decommissioning and integration (Gilburt, Edwards \& Murray, 2014).

Since I started to conduct this research there has been a shift in policy in the early intervention access waiting times standard. From 1 April 2016, the new access and waiting time standards for Early Intervention in Psychosis (EIP) services requires that $50 \%$ of people who experience a first episode of psychosis will be treated with a NICE approved care package within two weeks of referral. However, new analysis from HSJ (2018) had found nearly a quarter 
of clinical commissioning groups are spending less than the estimated sum needed to provide proper care to seriously ill mental health patients.

The Five Year Forward Review (2016) indicates that new leadership will be needed to make reality of new models of care. Emergent models of system leadership in the NHS have been identified (Fillingham \& Weir, 2014). These include applying the principles of complexity science and understanding the importance of learning by doing. System leadership is more likely to develop where there is a shared focus on a particular community and when effort is put into the development of many system leaders at multiple levels of the system. My analysis for this current study suggests that senior managers with direct experience of service delivery can best advocate for continuing organisational support for specialist services.

There is a need to ensure that there is a regular dialogue between various stakeholders such as practitioners and manager's to consider how to address the differences in culture and in language used by these groups. If commissioners are to lead the transformation of mental health services then there needs to be sufficient investment in their capacity and expertise to ensure that they have sufficient knowledge of specialist practice. There are implications too about the need for NHS decision-makers to communicate how and why they make decisions. An understanding of specialist practice should be paramount.

The way knowledge is used by managers and practitioners is important. Practitioners need to reflect on what kind of knowledge is to be valued within their own occupational and organisational context and why (Crilly et al., 2012). Clinical managers need to reflect about the difference and similarities between medical evidence (their original research culture) and management knowledge. Practitioner's knowledge transfer happens through experience in the field. Organisations need to build conditions in which such formal knowledge transfer can take place through a learning organisation or effective communities of practice.

The balance of power between clinical, hybrid managers, nurse managers and general managers may influence decisions, especially at local level. Nurse 
managers may be empowered to take initiative in facilitating innovation, or they may be given discrete and defined responsibilities in this area. Nurse managers may negotiate credible power, in a multi-professional setting by working in collaboration with a key clinician on a desired innovation. My results showed that nurses scored high on attitudes to openness to innovation compared to the psychiatrists. This however will indeed have implications for education and training of mental health clinicians. There are implications for clinical training on evidence based practice. Clearly there is value in both clinical and research evidence and training approach which utilises both, this may lead psychologists who are more and willing to bridge the gap (Dopson \& Fitzgerald, 2006). Changing complex practice behaviours is likely to be more difficult than changing relatively simple behaviours (Torrey \& Gorman, 2005). Panzano and Herman (2005) describe a simple formula for thinking about factors that determine the success of implementing new practices. Implementation success is viewed as 'a function of know-how, motivation, and the opportunity to learn' (2005, p. 265). Staff should acquire the opportunity to develop new knowledge and skills. They need to have either internal or external motivators to implement the behaviour change.

Implementation interventions can be useful and draw upon theory, evidence, and practical issues. Michie, Johnson \& Abraham et al., (2005) suggest that theory can be used to understand factors that might influence clinical behaviour change by targeting the behaviour and to clarify how such techniques might work.

Leadership is an important consideration and is central to realising systemic change (Ham, Edwards \& Brooke, 2013). This includes addressing stakeholder involvement and leadership both within organisations and as well as across organisations. Ensuring corporate commitment and leadership from trust boards and senior managers not only impacts positively on organisational implementation but can have knock-on effects on commissioner decisionmaking and support (Mears, Kendall \& Strathdee, 2008). 


\subsection{Suggestions for Future Research}

Future research should look into all stakeholders' views on implementing evidence based practices as this research has shown that a whole systems approach is required. Future implementation research should assess how the focus on therapist professional development within implementation efforts impact sustainment of EBPs at the provider and organizational levels. Recent research by Rye, Torres, Friborg, Skre, and Aarons (2017) has expanded the range of attitudes and resulted in the development of an eight domains dispersed across 35 new items namely EBPAS-50, which were conducted separately on US and Norwegian samples. This new scale introduces the following domains: limitations of EBP; the EBPs fit with values and needs of client and clinician; the negative perceptions of monitoring; the balance between perceptions of clinical skills and science as importance in service provision; time and administration burden with learning EBPs; job security related to expertise in EBP; perceived organisational support and positive perceptions of receiving feedback. I think researcher's wishing to conduct future studies could look at administering the EBPAS-50 with practitioners who implement evidence based practices.

The move towards service user involvement in research suggest the possibilities of examining implementation in more democratic ways that include people who will actually have experience of using services. 


\section{References}

Aarons, A. G \& Sawitzky, C. A. (2006). Organisational Climate partially mediates the effect of culture on work attitudes and staff turnover in Mental Health Services. Administration and Policy in Mental Health Services Research, 33(3), 289-301.

Aarons, A. G. (2004). Mental Health Provider Attitudes Toward Adoption of Evidence-Based Practice: The Evidence-Based Practice Attitude Scale (EBPAS). Mental Health Services Research, 6, (4), 61-74.

Aarons, G. A., \& Palinkas, L. A. (2007). Implementation of evidence-based practice in child welfare: Service provider perspectives. Administration and policy in mental health and mental health services research, 34(4), 411-419.

Aarons, G. A., Cafri, G., Lugo, L., \& Sawitzky, A. (2012). Expanding the domains of attitudes towards evidence-based practice: the evidence based practice attitude scale-50. Administration and policy in mental health and mental health services research, 39(5), 331-340.

Aarons, G. A., Glisson, C., Green, P. D., Hoagwood, K., Kelleher, K. J., \& Landsverk, J. A. (2012). The organizational social context of mental health services and clinician attitudes toward evidence-based practice: a United States national study. Implementation Science, 7(1), 56.

Aarons, G. A., Glisson, C., Hoagwood, K., Kelleher, K., Landsverk, J., \& Cafri, G. (2010). Psychometric properties and US National norms of the EvidenceBased Practice Attitude Scale (EBPAS). Psychological assessment, 22(2), 356

Aarons, G. A., Green, A. E., Trott, E., Willging, C. E., Torres, E. M., Ehrhart, M. G., \& Roesch, S. C. (2016). The Roles of System and Organizational Leadership in System-Wide Evidence-Based Intervention Sustainment: A Mixed-Method Study. Administration and Policy in Mental Health and Mental Health Services Research, 6(43), 991-1008.

Aarons, G. A., Hurlburt, M., \& Horwitz, S. M. (2011). Advancing a conceptual model of evidence-based practice implementation in public service sectors. Administration and Policy in Mental Health and Mental Health Services Research, 38(1), 4-23.

Aarons, G. A., Woodbridge, M., \& Carmazzi, A. (2003, March). Examining leadership, organizational climate and service quality in a children's system of care. In The 15th Annual Research Conference Proceedings, A System of Care for Children's Mental Health: Expanding the Research Base (pp. 15-18).

Ackroyd, S. (1996). Organization contra organizations: professions and organizational change in the United Kingdom. Organization Studies, 17(4), 599621. 
Adamson, V., Barrass, E., McConville, S., Irikok, C., Taylor, K., Pitt, S., ... \& Andrews, M. (2018). Implementing the access and waiting time standard for early intervention in psychosis in the United Kingdom: An evaluation of referrals and post-assessment outcomes over the first year of operation. Early intervention in psychiatry, 12(5), 979-986.

Addis, M. E., \& Krasnow, A. D. (2000). A national survey of practicing psychologists' attitudes toward psychotherapy treatment manuals. Journal of consulting and clinical psychology, 68(2), 331.

Addis, M. E., Wade, W. A., \& Hatgis, C. (1999). Barriers to dissemination of evidence-based practices: Addressing practitioners' concerns about manual-based psychotherapies. Clinical Psychology: Science and Practice, 6(4), 430-441.

Ajzen, I (1991). The theory of planned behaviour. Organizational Behaviour in Human Processes, 50, 179-211.

Ajzen, I., \& Fishbein, M. (1980). Understanding attitudes and predicting social behaviour. Englewood Cliffs, NJ: Prentice-Hall.

Allen, B., \& Armstrong, N. E. (2014). Burden of proof: The evidence clinicians require before implementing an intervention. Child and Adolescent Mental Health, 19(1), 52-56.

Anderson, N. R, \& West, M. A. (1998). Measuring climate for work group innovation: Development and validation of the team climate inventory. Journal of Organisational Behaviour, 19(3) 235-258.

Arnold, E. (2005). Managing human resources to improve employee retention. The Health Care Manager, 24(2), 132-140.

Ashkanasy, N.M., Broadfoot, C .E \& Falkus, S (2000a). Questionnaire Measures of Organisational Culture. In N.M. Ashkanasy, C.P.M. Wilderom, \& M.F. Peterson (Eds.). Handbook of organizational culture and climate (pp. 131146). Thousand Oaks, CA: Sage Publications.

Atkinson, P., and Hammersley, M. (1994). Ethnography and participant observation. In N. K. Denzin and Y. S. Lincoln (Eds.), Handbook of qualitative research (pp. 248-261) Thousand Oaks, CA: Sage.

Avolio BJ, Bass BM, Jung DI. Re-examining the components of transformational and transactional leadership using the Multifactor Leadership Questionnaire. Journal of Occupational and Organizational Psychology. 1999; 72:441-462.

Azorín, J. M., \& Cameron, R. (2010). The application of mixed methods in organisational research: A literature review. Electronic Journal of Business Research Methods, 8(2), 95-105. 
Bachmann, D., Elfrink, J., \& Vazzana, G. (1996). Tracking the progress of email vs. snail-mail. Marketing Research, 8, 31-35.

Bambling, M., King, R., Raue, P., Schweitzer, R., \& Lambert, W. (2006). Clinical supervision: Its influence on client-rated working alliance and client symptom reduction in the brief treatment of major depression. Psychotherapy Research, 16(03), 317-331

Bandura, A. (1988). Organizational application of social cognitive theory. Australian Journal of Management, 13(2), 257-302.

Barwick, M. A., Peters, J., \& Boydell, K. (2009). Getting to uptake: do communities of practice support the implementation of evidence-based practice?. Journal of the Canadian Academy of Child and Adolescent Psychiatry, 18(1), 16.

Bazeley, P. (2009). Integrating data analyses in mixed methods research. Journal of Mixed Methods Research 3(3), 203-207.

Bennett, S., Tooth, L., McKenna, K., Rodger, S., Strong, J., Ziviani, J., ... \& Gibson, L. (2003). Perceptions of evidence-based practice: A survey of Australian occupational therapists. Australian Occupational Therapy Journal, 50(1), 13-22.

Berger, R. (2015). Now I see it, now I don't: Researcher's position and reflexivity in qualitative research. Qualitative research, 15(2), 219-234.

Biesta, G., \& Burbules, N.C. (2003). Pragmatism and educational research. Lanham, MD: Rowman and Littlefield.

Bilsker, D., \& Goldner, E. M. (1999). Teaching evidence-based practice in mental health. Evidence Based Mental Health, (2), 68-69.

Bird, V., Premkumar, P., Kendall, T., Whittington, C., Mitchell, J., \& Kuipers, E. (2010). Early intervention services, cognitive-behavioural therapy and family intervention in early psychosis: systematic review. The British Journal of Psychiatry, 197(5), 350-356.

Birleson, P. (1999). Turning child and adolescent mental-health services into learning organizations. Clinical Child Psychology and Psychiatry, 4(2), 265-274.

Bond, G. R., Becker, D. R., \& Drake, R. E. (2011). Measurement of fidelity of implementation of evidence-based practices: Case example of the IPS Fidelity Scale. Clinical Psychology: Science and Practice, 18(2), 126-141.

Bond, G. R., Becker, D. R., Drake, R. E., Rapp, C. A., Meisler, N., Lehman, A. F., ... \& Blyler, C. R. (2001). Implementing Supported Employment as an Evidence-Based Practice. Psychiatric Services, 52(3), 313. 
Bond, G. R., Evans, L., Salyers, M. P., Williams, J., \& Kim, H. W. (2000). Measurement of fidelity in psychiatric rehabilitation. Mental health services research, 2(2), 75-87.

Botvin, G. J. (2004). Advancing prevention science and practice: Challenges, critical issues, and future directions. Prevention science, 5(1), 69-72.

Bowling, A. (2002). Research Methods in health. Investigating health and health services Second Edition. Open University Press.

Box, George E. P.; Norman R. Draper (1987). Empirical Model-Building and Response Surfaces, p. 424, Wiley.

Bradshaw, T., Butterworth, A., \& Mairs, H. (2007). Does structured clinical supervision during psychosocial intervention education enhance outcome for mental health nurses and the service users they work with? Journal of Psychiatric and Mental Health Nursing, 14(1), 4-12.

Brannen, J (1988). 'Research Note: The study of sensitive subjects'. The Sociological Review 36: 522-563.

Braun, V. \& Clarke, V. (2006). Using thematic analysis in psychology. Qualitative Research in Psychology, 3(2), 77-101.

Braun, V. \& Clarke, V. (2013) Successful Qualitative Research. Sage.

Breitenstein, S. M., Gross, D., Garvey, C. A., Hill, C., Fogg, L., \& Resnick, B. (2010). Implementation fidelity in community-based interventions. Research in nursing \& health, 33(2), 164-173.

Britten, N. (1995). Qualitative interviews in medical research, British Medical Journal, 311: 251-253.

Brown, J. S., \& Duguid, P. (2001). Knowledge and organization: A socialpractice perspective. Organization science, 12(2), 198-213.

Bryant, F. B. \& Yarnold, P. R. (1995). Principal components analysis and exploratory and confirmatory analysis, in Reading and Understanding Multivariate Statistics, L.G. Grimm and P.R. Yarnold (Eds.), American Psychological Association, Washington.

Bryman, A. (2001). Social Research Methods. Oxford. New York. Oxford University Press.

Bryman, A. (2012) Social Research Strategies Chapter 2. Bryman, A. (Ed), Social Research Methods $4^{\text {th }}$ edition (p 26). In Oxford University Press.

Burnard, P. (1994). The telephone interview as a data collection method. Nurse education today, 14(1), 67-72. 
Burns, B. J., Hoagwood, K., \& Mrazek, P. J. (1999). Effective treatment for mental disorders in children and adolescents. Clinical Child and Family Psychology Review, 2(4), 199-254.

Bush, P. W., Drake, R. E., Xie, H., McHugo, G. J., \& Haslett, W. R. (2009). The long-term impact of employment on mental health service use and costs for persons with severe mental illness. Psychiatric Services, 60(8), 1024-1031

Campbell, D. ( 2014). NHS treats mental health as 'second-class service' (24th July). The Guardian.

Candel, M. J., \& Pennings, J. M. (1999). Attitude-based models for binary choices: A test for choices involving an innovation. Journal of Economic Psychology, 20(5), 547-569.

Carr, E. C., \& Worth, A. (2001). The use of the telephone interview for research. NT research, 6(1), 511-524.

Chambers, D., Surender, R., Dopson, S., and Gabbay, J. (1999), 'Evaluation of the Welsh Clinical Effectiveness Initiative National Demonstration Projects; Final Report', Templeton College, Oxford and the Wessex Institute for Health Research and Development, University of Southampton.

Chapple, A. (1999). The use of telephone interviewing for qualitative research. Nurse Researcher, 6(3), 85-93.

Cherryholmes, C. .C (1992). Notes on pragmatism and scientific realism. Educational Researcher, 21, 13-17.

Cleary, M., \& Freeman, A. (2006). Fostering a culture of support in mental health settings: Alternatives to traditional models of clinical supervision. Issues in Mental Health Nursing, 27(9), 985-1000.

Cleary, M., Hunt, G. E., Walter, G., \& Jackson, D. (2010). Fostering real-world clinical mental health research. Journal of clinical nursing, 19(23-24), 34533458.

Cohen, L. H., Sargent, M. M., \& Sechrest, L. B. (1986). Use of psychotherapy research by professional psychologists. American psychologist, 41(2), 198.

Cohen,. M.D. (1996). Individual learning and organisational routine', in M.D. Cohen and L.S. Sproull (eds) Organisational learning, London: Sage Publications, pp188-94.

Coiera, E. (2011). Why system inertia makes health reform so difficult. BMJ: British Medical Journal, 342.

Colquhoun, H. L., Squires, J. E., Kolehmainen, N., Fraser, C., \& Grimshaw, J. M. (2017). Methods for designing interventions to change healthcare 
professionals' behaviour: a systematic review. Implementation Science, 12(1), 30 .

Cook, T.D. (1979). Quasi-experimentation: Design and analysis issues for field settings. Boston: Houghton Mifflin.

Cortina M. L. (2005). Model Selection. In B.S.Everitt \& D.C.Howell (Eds.), Encyclopedia of Statistics in Behavioural Science (pp. 1251-1253). Wiley.

Court, A. J., Cooke, A., \& Scrivener, A. (2017). They're NICE and Neat, but Are They Useful? A Grounded Theory of Clinical Psychologists' Beliefs About and Use of NICE Guidelines. Clinical psychology \& psychotherapy, 24(4), 899-910.

Craig, T. K., Garety, P., Power, P., Rahaman, N., Colbert, S., Fornells-Ambrojo, M., \& Dunn, G. (2004). The Lambeth Early Onset (LEO) Team: randomised controlled trial of the effectiveness of specialised care for early psychosis. Bmj, 329(7474), 1067.

Creswell, J. W., \& Plano Clark, V. (2011). Designing and conducting mixed methods research Second Edition. Thousand Oaks, CA: Sage Publications Inc.

Creswell, J. W., \& Plano Clark, V. (2007). Designing and conducting mixed methods research. Thousand Oaks, CA: Sage.

Creswell, J. W., Plano Clark, V. L., Gutmann, M. L., \& Hanson, W. E. (2003). Advanced mixed methods research designs. Handbook of mixed methods in social and behavioral research, 209, 240.

Crilly T, Jashapara A, Ferlie E. Research Utilisation and Knolwedge Mobilisation. A scoping Review of the Literature. Service Delivery Organisation. Draft Report 3 July 2009. SDO Project Reference 08/1801/220. Retrieved from CiteSeerX website:

http://citeseerx.ist.psu.edu/viewdoc/download?doi=10.1.1.385.625\&rep=rep1\&ty pe $=$ pdf

Csillag, C., Nordentoft, M., Mizuno, M., Jones, P. B., Killackey, E., Taylor, M., ... \& McDaid, D. (2016). Early intervention services in psychosis: from evidence to wide implementation. Early intervention in psychiatry, 10(6), 540-546.

Cunningham, R. (2013) Dissemination methods and attitudes to family intervention for psychosis in trainee clinical psychologists. (PhD thesis, University of Liverpool). Retrieved from EThoS database (uk.bl.ethos.592827).

Damanpour, F. (1991). Organizational Innovation: A Meta-analysis of effects of determinants and moderators. Academy of Management Journal, 34(3), 555590.

Dane, A. V., \& Schneider, B. H. (1998). Program integrity in primary and early secondary prevention: are implementation effects out of control?.Clinical psychology review, 18(1), 23-45. 
Dark, F., Whiteford, H., Ashkanasy, N. M., Harvey, C., Crompton, D., \& Newman, E. (2015). Implementing cognitive therapies into routine psychosis care: organisational foundations. BMC health services research, 15(1), 310.

Davies, P., Walker, A. E., \& Grimshaw, J. M. (2010). A systematic review of the use of theory in the design of guideline dissemination and implementation strategies and interpretation of the results of rigorous evaluations. Implementation Science, 5(1), 1.

Davies, T.O. H., Nutley, M. S., \& Smith, C. P. (2004). What Works? Evidencebased policy and practice in public services. The Policy Press.

Day, E. A., Arthur Jr, W., \& Gettman, D. (2001). Knowledge structures and the acquisition of a complex skill. Journal of applied psychology, 86(5), 1022.

De Heer, W., de Leeuw, E. D., \& van der Zouwen, J. (1999). Methodological issues in survey research: a historical review. Bulletin of Sociological Methodology/Bulletin de Méthodologie Sociologique, 64(1), 25-48.

Department of Health (2001). The Mental Health Policy Implementation Guideline. Retrieved from DoH website:

http://webarchive.nationalarchives.gov.uk/+/http://www.dh.gov.uk/en/Publication sandstatistics/Publications/PublicationsPolicyAndGuidance/DH_4009350

Department of Health (2016). 'Prime Minister pledges a revolution in mental health treatment'. Press release, 11 January. Retrieved from DoH website: www.gov.uk/government/news/prime-minister-pledges-arevolution-in-mentalhealth-treatment.

Department of Health 2000. The NHS Plan. A plan for investment. A plan for reform, London, HMSO. Retrieved from DoH website:

http://webarchive.nationalarchives.gov.uk/+/http://www.dh.gov.uk/en/Publication sandstatistics/Publications/PublicationsPolicyAndGuidance/DH_4002960.

Department of Health. (1999). The National Service Framework for Mental Health. London: Department of Health. Retrieved from Department of Health website: https://www.gov.uk/government/publications/quality-standards-formental-health-services.

Department of Health. (2009). Departmental Report 2009: The Health and Personal Social Services Programmes. Retrieved from Department of Health website:

https://www.gov.uk/government/uploads/system/uploads/attachment_data/file/2 28879/7593.pdf.

Department of Health. (2010) The Caldicott Manual Guardian. UK Council of Caldicott Guardians. Retrieved from the UK Council Caldicott Guardians website: https://www.gov.uk/government/groups/uk-caldicott-guardian-council. 
Dewey, J (1925). Experience and philosophical method. In J and J Stuhr (Ed). Pragmatism and classical. America philosophy. Second Edition pp 460/471. New York and Oxford University Press.

Dijkstra, R., Wensing, M., Thomas, R., Akkermans, R., Braspenning, J., Grimshaw, J., \& Grol, R. (2006). The relationship between organisational characteristics and the effects of clinical guidelines on medical performance in hospitals, a meta-analysis. BMC Health Services Research, 6(1), 53.

Docherty, M., \& Thornicroft, G. (2015). Specialist mental health services in England in 2014: overview of funding, access and levels of care. International journal of mental health systems, 9(1), 34.

Dodgson, G., \& McGowan, S. (2010). Early intervention service models. Promoting Recovery in Early Psychosis: A Practice Manual, 45-52.

Dopson, S., \& Fitzgerald, L. (2005). Knowledge to action? Evidence-based health care in context. Oxford University Press.

Dopson, S. U. E., \& Fitzgerald, L. (2006). The role of the middle manager in the implementation of evidence-based health care. Journal of nursing management, 14(1), 43-51.

Dopson, S., \& Mark, A. L. (2003). Leading health care organizations. Palgrave Macmillan.

Dopson, S., FitzGerald, L., Ferlie, E., Gabbay, J., \& Locock, L. (2002). No magic targets! Changing clinical practice to become more evidence based. Health care management review, 27(3), 35-47.

Drake, E. R, Torrey, C. W., McHugo, J. G. (1998). Strategies for implementing evidence-based practice in routine mental health settings. Evidence Based Mental Health, 6(1), 6-7.

Drake, R. E., Goldman, H. H., Leff, H. S., Lehman, A. F., Dixon, L., Mueser, K. T., \& Torrey, W. C. (2001). Implementing evidence-based practices in routine mental health service settings. Psychiatric services, 52(2), 179-182.

Dusenbury, L., Brannigan, R., Falco, M., \& Hansen, W. B. (2003). A review of research on fidelity of implementation: implications for drug abuse prevention in school settings. Health education research, 18(2), 237-256.

Early Intervention in Psychosis IRIS Network. (2012) IRIS Guidelines Update IRIS Initiative Ltd. Retrieved from the iris-initiative website: www.irisinitiaitve.org.uk.

Eccles, M. P., \& Mittman, B. S. (2006). Welcome to implementation science. Implementation Science, 1(1), 1. 
Edwards, J., \& McGorry, P. D. (2002). Implementing early intervention in psychosis: A guide to establishing psychosis services. CRC Press.

Eraut, M. (1985). Knowledge creation and knowledge use in professional contexts. Studies in Higher Education, 10(2), 117-133.

Eraut, M. (2000). Non-formal learning and tacit knowledge in professional work. British journal of educational psychology, 70(1), 113-136.

Everett, B., \& Howell., D (2005) Encyclopaedia of Statistics in Behavioural Science, Wiley.

Fabrigar, L. R., Wegener, D. T., MacCallum, R. C., \& Strahan, E. J. (1999). Evaluating the use of exploratory factor analysis in psychological research. Psychological methods, 4(3), 272.

Fenton, M., Timimi, H., Chalmers, I. (2006). Development of the Database of Uncertainties about the Effects of Treatments (DUETs) A resource to help prioritise new research. Retrieved from James Lind Alliance website: http://www.jla.nihr.ac.uk/news-and-publications/downloads/2007-2008-DUETsDevelopment-Report.pdf

Ferlie, E., Crilly, T., Jashapara, A., \& Peckham, A. (2012). Knowledge mobilisation in healthcare: a critical review of health sector and generic management literature. Social science \& medicine, 74(8), 1297-1304.

Ferlie, E., Dopson, S. U. E., Fitzgerald, L., \& Locock, L. (2009). Renewing policy to support evidence-based health care. Public Administration, 87(4), 837-852. Ferrari, R. (2015). Writing narrative style literature reviews. Medical Writing, 24(4), 230-235.

Fielding, N., \& Fielding , J. (1986) Linking data: The articulation of qualitative and quantitative methods in social research. Beverly Hills, CA: Sage.

Fillingham D, Weir B (2014). System leadership: lessons and learning from AQuA's Integrated Care Discovery Communities. London: The King's Fund. Available at: www.kingsfund.org.uk/publications/ system-leadership (Accessed on 19 November 2018).

Firn, M., Hindhaugh, K., Hubbeling, D., Davies, G., Jones, B., \& White, S. J. (2013). A dismantling study of assertive outreach services: comparing activity and outcomes following replacement with the FACT model. Social psychiatry and psychiatric epidemiology, 48(6), 997-1003.

Fishbein, M., \& Ajzen, I. (2011). Predicting and changing behaviour: The reasoned action approach. Taylor \& Francis.

Fishbein, M., Jaccard, J., Davidson, A. R., Ajzen, I., \& Loken, B. (1980). Predicting and understanding family planning behaviors. In Understanding attitudes and predicting social behavior. Prentice Hall. 
Fixsen, D. L., Naoom, S. F., Blase, K. A., \& Friedman, R. M. (2005). Implementation research: a synthesis of the literature.

Fontana, A., \& Frey, J. H. (2005). The interview: From neutral stance to political involvement. The Sage handbook of qualitative research., 3rd ed.

Fowler, D., Hodgekins, J., Howells, L., Millward, M., Ivins, A., Taylor, G., ... \& Macmillan, I. (2009). Can targeted early intervention improve functional recovery in psychosis? A historical control evaluation of the effectiveness of different models of early intervention service provision in Norfolk 19982007. Early Intervention in Psychiatry, 3(4), 282-288.

Fowler, D., Hodgekins, J., Howells, L., Millward, M., Ivins, A., Taylor, G., \& Frambach, R. T., \& Schillewaert, N. (2002). Organizational innovation adoption: A multi-level framework of determinants and opportunities for future research. Journal of Business Research, 55(2), 163-176.

Freitas, G. J. (2002). The impact of psychotherapy supervision on client outcome: A critical examination of 2 decades of research. Psychotherapy: Theory, Research, Practice, Training, 39(4), 354.

Friis, S. (2010). Early specialised treatment for first-episode psychosis: does it make a difference? The British journal of psychiatry, 196(5), 339-340.

Gafoor, R., Nitsch, D., McCrone, P., Craig, T. K., Garety, P. A., Power, P., \& McGuire, P. (2010). Effect of early intervention on 5-year outcome in nonaffective psychosis. The British Journal of Psychiatry, 196(5), 372-376.

Gallo, K. P., \& Barlow, D. H. (2012). Factors involved in clinician adoption and nonadoption of evidence-based interventions in mental health. Clinical Psychology: Science and Practice, 19(1), 93-106.

Garland, A. F., Kruse, M., \& Aarons, G. A. (2003). Clinicians and outcome measurement: What's the use? The journal of Behavioural Health Services \& Research, 30(4), 393-405.

Garman, A. N., Davis-Lenane, D., \& Corrigan, P. W. (2003). Factor structure of the transformational leadership model in human service teams. Journal of Organizational Behaviour, 24(6), 803-812.

Gibbons, M., Limoges, C., Nowotny, H., Schwatzman, S., Scott, P. and Trow, M. (1994). The production of knowledge, London: Sage Publications.

Gilbert, J. (2003). Between a rock and a hard place? Training and personal development issues for mental health service managers. Mental Health Practice, 6(10), 31-33. 
Gilbert, J. (2003). Between a rock and a hard place? Training and personal development issues for mental health service managers. Mental Health Practice, 6(10), 31-33.

Gilburt, H., Edwards, N., \& Murray, R. (2014). Transforming Mental Health: a plan of action for London. King's Fund.

Gillam., T (2010) Clinical Supervision in early intervention teams in French P., Smith., J., Shiers., D., Reed., M., Rayne., M (Eds.), Promoting Recovery in Early Psychosis (p281). Wiley-Blackwell.

Glaser, B.G., \& Strauss, A. L. (1965). The discovery of grounded theory: Strategies for qualitative research. Chicago: Aldine.

Glasgow, R. E., \& Chambers, D. (2012). Developing robust, sustainable, implementation systems using rigorous, rapid and relevant science. Clinical and Translational Science, 5(1), 48-55.

Glasgow, R. E., Lichtenstein, E., \& Marcus, A. C. (2003). Why don't we see more translation of health promotion research to practice? Rethinking the efficacy-to-effectiveness transition. American journal of public health, 93(8), 1261-1267.

Glisson, C. (2002). The organizational context of children's mental health services. Clinical child and family psychology review, 5(4), 233-253.

Glisson, C., \& Durick, M. (1988). Predictors of job satisfaction and organizational commitment in human service organizations. Administrative science quarterly, 61-81

Glisson, C., \& James, L. R. (2002). The cross-level effects of culture and climate in human service teams. Journal of Organizational Behaviour, 23(6), 767-794.

Glisson, C., \& Schoenwald, S. K. (2005). The ARC organizational and community intervention strategy for implementing evidence-based children's mental health treatments. Mental health services research, 7(4), 243-259.

Glisson, C., Landsverk, J., Schoenwald, S., Kelleher, K., Hoagwood, K. E., Mayberg, S., ... \& Research Network on Youth Mental Health. (2008).

Assessing the organizational social context (OSC) of mental health services: Implications for research and practice. Administration and Policy in Mental Health and Mental Health Services Research, 35(1-2), 98.

Gorard, S., \& Taylor, C. (2004). Combining methods in educational and social research. Buckingham, UK: Open University Press.

Gøtzsche, P. C. (2000). Why we need a broad perspective on meta-analysis: It may be crucially important for patients. BMJ: British Medical Journal, 321(7261), 585. 
Graham, I. D., \& Tetroe, J. (2009). Planned action theories. Knowledge translation in health care: Moving from evidence to practice, 185-195

Grawe, R. W., Falloon, I. R. H., Widen, J. H., \& Skogvoll, E. (2006). Two years of continued early treatment for recent-onset schizophrenia: a randomised controlled study. Acta Psychiatrica Scandinavica, 114(5), 328-336.

Greene, J. C., Caracelli, V. J., and Graham, W. F. (1989). Towards a conceptual framework for mixed method evaluation designs. Educational Evaluation and Policy Analysis, 11, 255-274.

Greenhalgh, T., Robert, G., Macfarlane, F., Bate, P., \& Kyriakidou, O. (2004). Diffusion of innovations in service organizations: systematic review and recommendations. The Milbank Quarterly, 82(4), 581-629.

Greenhalgh, T., Robert, G., Macfarlane, F., Bate, P., Kyriakidou, O., \& Peacock, R. (2005). Storylines of research in diffusion of innovation: a meta-narrative approach to systematic review. Social science \& medicine, 61(2), 417-430.

Greenhalgh, T., Thorne, S., \& Malterud, K. (2018). Time to challenge the spurious hierarchy of systematic over narrative reviews?. European journal of clinical investigation, 48(6), e12931.

Grimshaw, J., Eccles, M., \& Tetroe, J. (2004). Implementing clinical guidelines: current evidence and future implications. Journal of Continuing Education in the Health Professions, 24(S1).

Grol, R., \& Wensing, M. (2004). What drives change? Barriers to and incentives for achieving evidence-based practice. Medical Journal of Australia, 180(6), S57.

Grol, R., Wensing, M., Bosch, M., Hulscher, M., \& Eccles, M. (2013). Theories on implementation of change in healthcare. Improving Patient Care: The Implementation of Change in Health Care, Second Edition, 18-39.

Guba, E. G., \& Lincoln, Y. S. (1981). Effective evaluation: Improving the usefulness of evaluation results through responsive and naturalistic approaches. Jossey-Bass.

Guest, G., Bunce, A., \& Johnson, L. (2006). How many interviews are enough? An experiment with data saturation and variability. Field methods, 18(1), 59-82.

Guy, A., Thomas, R., Stephenson, S., \& Loewenthal, D. (2011). NICE under scrutiny. Retrieved from UKCP website: http://www. psychotherapy. org. uk/nice_campaign_2011. html.

Haddock, G., Eisner, E., Boone, C., Davies, G., Coogan, C., \& Barrowclough, C. (2014). An investigation of the implementation of NICE-recommended CBT 
interventions for people with schizophrenia. Journal of Mental Health, 23(4), 162-165.

Haines, A., \& Donald, A. (1998). Looking forward: Making better use of research findings. BMJ: British Medical Journal, 317(7150), 72.

Haines, A., Kuruvilla, S., \& Borchert, M. (2004). Bridging the implementation gap between knowledge and action for health. Bulletin of the World Health Organization, 82(10), 724-731.

Ham ,C. \& Murray, R. (2015). Implementing the NHS Five Year Forward Review: aligning policies with the plan. London: The King's Fund. Retrieved November 162018 from Kings fund website:

https://www.kingsfund.org.uk/sites/default/files/field/field_publication_file/implem enting-the-nhs-five-year-forward-view-kingsfund-feb15.pdf

Ham C, Edwards N, Brooke B (2013). Leading health care in London: time for a radical response. London: The King's Fund. Retrieved from the Kings Fund website: www.kingsfund.org.uk/publications/leading-health-carelondon (Accessed on 16 September 2017).

Hammersley, M. (2009). Against the ethicists: on the evils of ethical regulation. International Journal of Social Research Methodology, 12(3), 211-225.

Hayes, J. A., \& Wall, T. N. (1998). What influences clinicians' responsibility attributions? The role of problem type, theoretical orientation, and client attribution. Journal of Social and Clinical Psychology, 17(1), 69-74.

Health Service Journal (2018) Quarter of CCGs fall below psychosis spend benchmark, by Joe Gammie. Retrieved October 192018 from HSJ website, https://www.hsj.co.uk/quality-and-performance/quarter-of-ccgs-fall-belowpsychosis-spend-benchmark/7023521.article\#commentsJump.

Henwood, K., \& Pidgeon, N. (1994). Beyond the qualitative paradigm: A framework for introducing diversity within qualitative psychology. Journal of Community \& Applied Social Psychology, 4(4), 225-238.

Hill, L. G., Maucione, K., \& Hood, B. K. (2007). A focused approach to assessing program fidelity. Prevention Science, 8(1), 25-34.

HM Government 2017. The Government's Response to the Five Year Forward Review for Mental Health. Retrieved from September 272018 from, https://assets.publishing.service.gov.uk/government/uploads/system/uploads/att achment_data/file/582120/FYFV_mental_health_government_response.pdf.

HM Government 2009. New Horizons: a shared vision for mental health, London, Department of Health. Retrieved September 272018 from, http://webarchive.nationalarchives.gov.uk/+/http://www.dh.gov.uk/en/Consultatio ns/Liveconsultations/DH_103144. 
HM Government 2011. No health without mental health: A cross-government mental health outcomes strategy for people of all ages, London, Department of Health. Retrieved September 272018 from, https://www.gov.uk/government/publications/no-health-without-mental-health-across-government-mental-health-outcomes-strategy-for-people-of-all-ages-acall-to-action.

Hoagwood, K., Burns, B. J., Kiser, L., Ringeisen, H., \& Schoenwald, S. K. (2001). Evidence-based practice in child and adolescent mental health services. Psychiatric Services, 52(9), 1179-1189.

Hodson, R. (2003). Leading the drive for evidence based practice in services for children and families: Summary report of a study conducted for research in practice. UK: Research in Practice. Retrieved May 292018 from, http://www.rip.org

Howe, K. R (2004). Against the quantitative-qualitative incompatibility thesis or dogmas die hard. Educational Researcher, 17, 10-16.

Howe, K. R. (1988). Against the quantitative-qualitative incompatibility thesis or dogmas die hard. Educational Researcher, 17 10-16.

Huxley, P. J, Evans, S., Baker, C. M., White, J., Madge, S., Onyett, S., Gould, N. (2012). The social care component of multidisciplinary mental health teams: a review and national study. Journal of Health Services Research Policy, Vol 17 (2) pp23-29.

Huxley, P. J, Evans, S., Baker, C. M., White, J., Philpin, S. A., Onyett, S., Gould, N., Integration of social care staff within community mental health teams. Final report. NIHR Service Delivery and Organisation Programme; March 2011. Retrieved June 2017 from SDO website, http://www.nets.nihr.ac.uk/projects/hsdr/081619114.

Huxley, P. J, Evans, S., Baker, C. M., White, J., Philpin, S. A., Onyett, S., Gould, N., Integration of social care staff within community mental health teams. Final report. NIHR Service Delivery and Organisation Programme; March 2011. Retrieved March 112017 from SDO website, http://www.netscc.ac.uk/hsdr/files/project/SDO_ES_08-1619-114_V01.pdf

Huxley, P. J, Evans, S., R, Mayo., Ball, W., Maegusuku-Hewett, T. (2009). Social Care Research Priorities and Capacity in Wales. A Consultation Exercise. Final Report Swansea University. Centre for Social Work and Social Care. Retrieved March 112017 from Wales NHS website, http: http://www.wales.nhs.uk/sites3/Documents/952/Huxley\%20Report\%202009.pdf

IBM SPSS Statistics for Windows, Version 22.0. Armonk, NY: IBM Corp. IBM Corp. Released 2013.

Ince, P., Haddock, G., \& Tai, S. (2016). A systematic review of the implementation of recommended psychological interventions for schizophrenia: 
rates, barriers, and improvement strategies. Psychology and Psychotherapy: Theory, Research and Practice, 89(3), 324-350.

IRIS (2010). Early Intervention for Psychosis. A briefing for service planners.

IRIS (2012). IRIS Guidelines update, Birmingham, IRIS Initiative Ltd.

Irvine, A. (2011). Duration, dominance and depth in telephone and face-to-face interviews: A comparative exploration. International Journal of Qualitative Methods, 10(3), 202-220.

Jackson, H., McGorry, P., Edwards, J., Hulbert, C., Henry, L., Harrigan, S., ... \& Killackey, E. (2005). A controlled trial of cognitively oriented psychotherapy for early psychosis (COPE) with four-year follow-up readmission data. Psychological medicine, 35(9), 1295-1306.

Jensen-Doss, A., Hawley, K. M., Lopez, M., \& Osterberg, L. D. (2009). Using evidence-based treatments: The experiences of youth providers working under a mandate. Professional Psychology: Research and Practice, 40(4), 417.

Johnson, R. B., \& Onwuegbuzie, A. (2004). Mixed methods research: A research paradigm whose time has come. Educational Researcher, 33(7), 1426.

Jung, T., Scott, T., Davies, H. T., Bower, P., Whalley, D., McNally, R., \& Mannion, R. (2009). Instruments for exploring organizational culture: A review of the literature. Public administration review, 69(6), 1087-1096.

Kane, B. (2002) Social Capital, health and economy in South Yorkshire coalfield communities, In: Bauld, L., Judge, K., (eds). „Learning from Health Action Zones", 187-198. Chichester: Aeneas Press.

Kauth, M. R., Sullivan, G., Cully, J., \& Blevins, D. (2011). Facilitating practice changes in mental health clinics: A guide for implementation development in health care systems. Psychological services, 8(1), 36.

Kish, L. (1949). A procedure for objective respondent selection within the household. Journal of the American Statistical Association, 44(247), 380-387.

Konnerup, M., \& Kongsted, H. C. (2012). Do Cochrane reviews provide a good model for social science? The role of observational studies in systematic reviews. Evidence \& Policy: A Journal of Research, Debate and Practice, 8(1), 79-96.

Kraut, R., Olson, J., Banaji, M., Bruckman, A., Cohen, J., \& Couper, M. (2004). Psychological research online: report of Board of Scientific Affairs' Advisory Group on the Conduct of Research on the Internet. American psychologist, 59(2), 105. 
Kuhn, T. S. (1962). The structure of scientific revolutions $\left(2^{\text {nd }}\right.$ ed.). Chicago: University of Chicago Press.

Kuipers, E., Garety, P., Fowler, D., Freeman, D., Dunn, G., \& Bebbington, P. (2006). Cognitive, emotional, and social processes in psychosis: refining cognitive behavioral therapy for persistent positive symptoms. Schizophrenia bulletin, 32(suppl_1), S24-S31.

Kuipers, E., Holloway, F., Rabe-Hesketh, S., \& Tennakoon, L. (2004). An RCT of Early Intervention in Psychosis: Croydon Outreach and Assertive Support Team (COAST). Social psychiatry and psychiatric epidemiology, 39(5), 358363.

Learmonth, M. (1999). The National Health Service manager, engineer and father? A deconstruction. Journal of Management Studies, 36(7), 999-1012.

Leavey, G., Gulamhussein, S., Papadopoulos, C., Johnson-Sabine, E., Blizard, B., \& King, M. (2004). A randomized controlled trial of a brief intervention for families of patients with a first episode of psychosis. Psychological medicine, 34(3), 423-431

Lecomte, T., Leclerc, C., Corbiere, M., Wykes, T., Wallace, C. J., \& Spidel, A. (2008). Group Cognitive Behavior Therapy or Social Skills Training for Individuals With a Recent Onset of Psychosis?: Results of a Randomized Controlled Trial. The Journal of nervous and mental disease, 196(12), 866-875.

Leff J. Family management of schizophrenia. In: Shriqui CL, Nasrallah HA editor(s). Contemporary Issues in the Treatment of Schizophrenia. Washington DC: American Psychiatric Press, 1995: 683-702.

Lester, H., Birchwood, M., Bryan, S., England, E., Rogers, H., \& Sirvastava, N. (2009). Development and implementation of early intervention services for young people with psychosis: case study. The British Journal of Psychiatry, 194(5), 446-450.

Lieberman, J. A. (1993). Prediction of outcome in first-episode schizophrenia. The Journal of clinical psychiatry.

Llewellyn, S. (2001). Two-way windows': clinicians as medical managers. Organization Studies, 22(4), 593-623.

Llieva, J., Baron, S., \& Healey, N. M. (2002). Online surveys in marketing research: Pros and cons. International Journal of Market Research, 44(3), 361.

Lloyd, K. and White J. (2011). Democratising Clinical Research. Nature Vol 474 pp277-278.

Lloyd, K. and White, J. (2012). Patients Research Priorities get Funded. Nature vol 487, p432. 
Lloyd., C., King R., Deane P., \& Gournay K. (2009). Clinical management in mental health services. Wiley-Blackwell.

Locock, L., \& Dopson, S. (2012). Shaping strategic change: changing the way organisational change was researched in the NHS. Shaping Health Policy: Case Study Methods and Analysis, 201.

Locock, L., Dopson, S., Chambers, D., \& Gabbay, J. (2001). Understanding the role of opinion leaders in improving clinical effectiveness. Social science \& medicine, 53(6), 745-757.

Long, J. C., Cunningham, F. C., \& Braithwaite, J. (2013). Bridges, brokers and boundary spanners in collaborative networks: a systematic review. BMC health services research, 13(1), 158.

Loy, J. W., Jr. (1968). Social psychological characteristics of innovators. American Sociological Review, 34, 73-82.

Lutgens, D., Gariepy, G., \& Malla, A. (2017). Psychological and psychosocial interventions for negative symptoms in psychosis: systematic review and metaanalysis. The British Journal of Psychiatry, bjp-bp

Mabry., L (2009) Case study in social research Chapter 13. Alasuutari,. P., Bickman., \& Brannen., J (Eds.). (2009). The sage handbook of Social Research Methods. Sage.

MacCallum, R.C. (1995). Model specification: procedures, strategies, and related issues, in structural equation modelling: Concepts, Issues, and Applications, R.H. Hoyle, ed., Sage Publications, Thousand Oaks, pp16-36.

Macmillan, I. (2009). Can targeted early intervention improve functional recovery in psychosis? A historical control evaluation of the effectiveness of different models of early intervention service provision in Norfolk 1998-2007. Early intervention in psychiatry, 3(4), 282-288. DOI: 10.1111/j.17517893.2009.00146.x

Macmillan, I. (2009). Can targeted early intervention improve functional recovery in psychosis? A historical control evaluation of the effectiveness of different models of early intervention service provision in Norfolk 1998-2007. Early intervention in psychiatry, 3(4), 282-288.

Madill, A., Jordan, A., \& Shirley, C. (2000). Objectivity and reliability in qualitative analysis: Realist, contextualist and radical constructionist epistemologies. British journal of psychology, 91(1), 1-20.

Magliano, I., Fiorillo, A., Fadden, G., Gair, F., Economou, M., Kallert, t., \& Palma-Crespo, A. (2005). Effectiveness of a psychoeducational intervention for families of patients with schizophrenia: preliminary results of a study funded by the European Commission. World Psychiatry, 4(1), 45. 
March, J. G., \& Olsen, J. P. (1976). Organizational Learning and the Ambiguity of the Past. Ambiguity and choice in organizations, 408.

Mari, J. D. J., \& Streiner, D. L. (1994). An overview of family interventions and relapse on schizophrenia: meta-analysis of research findings. Psychological medicine, 24(3), 565-578.

Marshall, M., \& Rathbone, J. (2011). Early intervention for psychosis. Cochrane Database of Systematic Reviews, (6). doi/10.1002/14651858.CD004718.pub3.

Marshall, M., Husain, N., Bork, N., Chaudhry, I. B., Lester, H., Everard, L., ... \& Fowler, D. (2014). Impact of early intervention services on duration of untreated psychosis: data from the National EDEN prospective cohort study. Schizophrenia research, 159(1), 1-6.

Matrix Cymru (2017). Guidance for delivering Evidence Based Psychological Therapies in Wales. National Psychological Therapies Management Committee, NHS Wales. Retrieved November 102018 from the 1000 Lives website, http://www.1000livesplus.wales.nhs.uk/sitesplus/documents/1011/Matrics\%20C ymru\%20\%28CM\%20design\%20-\%20DRAFT\%2015\%29.pdf.

May, T. (2001). Social research, issues, methods and process. $2^{\text {nd }} \mathrm{ed}$. California: Open University.

Mazzucchelli, T. G., \& Sanders, M. R. (2010). Facilitating practitioner flexibility within an empirically supported intervention: Lessons from a system of parenting support. Clinical Psychology: Science and Practice, 17(3), 238-252.

McCaskey, M. (1988) 'The challenge of managing ambiguity and change' in managing and change. L.R. Pondy, R.J Boland and H. Thomas (eds.), Wiley 1 17 Chichester.

McCaskey, M. B. (1988). The challenge of managing ambiguity and change (pp. 1-15). New York: John Wiley \& Sons.

McCoyd, J. L., \& Kerson, T. S. (2006). Conducting intensive interviews using email: A serendipitous comparative opportunity. Qualitative Social Work, 5(3), 389-406.

McCrae, R. R., \& Costa, P. T. (2003). Personality in adulthood: A five-factor theory perspective. Guilford Press.

McCrone, P., Craig, T. K., Power, P., \& Garety, P. A. (2010). Cost-effectiveness of an early intervention service for people with psychosis. The British Journal of Psychiatry, 196(5), 377-382.

McDaid, D., Park A-La., Lemmi V., Adelaja B., Knapp M. (2016) Growth in the use of early intervention for psychosis services: An opportunity to promote recovery amid concerns on health care sustainability. LSE Personal Social Services Research Unit. 
McGorry, P. D. (2007). The specialist youth mental health model: strengthening the weakest link in the public mental health system. Medical Journal of Australia, 187(7), S53.

McGorry, P. D., \& Yung, A. R. (2003). Early intervention in psychosis: an overdue reform. Australian and New Zealand Journal of Psychiatry, 37(4), 393398.

McGorry, P. D., Purcell, R., Hickie, I. B., \& Jorm, A. F. (2007). Investing in youth mental health is a best buy. Medical Journal of Australia, 187(7), S5.

McGovern, M. P., Fox, T. S., Xie, H., \& Drake, R. E. (2004). A survey of clinical practices and readiness to adopt evidence-based practices: Dissemination research in an addiction treatment system. Journal of substance abuse treatment, 26(4), 305-312.

McHugh, R. K., \& Barlow, D. H. (2010). The dissemination and implementation of evidence-based psychological treatments: a review of current efforts. American Psychologist, 65(2), 73.

McHugo, G. J., Drake, R. E., Xie, H., \& Bond, G. R. (2012). A 10-year study of steady employment and non-vocational outcomes among people with serious mental illness and co-occurring substance use disorders. Schizophrenia Research, 138(2), 233-239

McNicoll, A. 2014b. Rising caseloads and cuts hit acclaimed early intervention psychosis services. Community Care.

Mears A, Kendall T, Strathdee G, Sinfield G, Aldridge I (2008). 'Progress on NICE guideline implementation in mental health trusts: meta-analyses'. The Psychiatric Bulletin, vol 32, pp 383-87.

Meek, V. L. (1988). Organizational culture: Origins and weaknesses. Organization studies, 9(4), 453-473.

Menard, S., (2010). Logistic Regression. From Introductory to Advanced Concepts and Applications. Sage Publications.

Mental Health Strategies 2012. 2011/2012 national survey of investment in adult mental health services, Manchester, Mental Health Strategies Retrieved 17 October 2018 from Government website, https://www.gov.uk/government/news/national-survey-of-investment-in-mentalhealth-services-for-2011-12

Mental Health Taskforce (2016). The Five Year Forward Review. Retrieved October 272018 from NHS website, https://www.england.nhs.uk/wpcontent/uploads/2016/02/Mental-Health-Taskforce-FYFV-final.pdf 
Metcalfe, L. \& Richards, S. (1990). Improving public management, London: Sage Publications.

Michie, S., Johnston, M., Abraham, C., Lawton, R., Parker, D., \& Walker, A. (2005). Making psychological theory useful for implementing evidence based practice: a consensus approach. BMJ Quality \& Safety, 14(1), 26-33.Michie, S., Pilling, S., Garety, P., Whitty, P., Eccles, M. P., Johnston, M., \& Simmons, J. (2007). Difficulties implementing a mental health guideline: an exploratory investigation using psychological theory. Implementation Science, 2(1), 8.

Mihalic, S. (2004). The importance of implementation fidelity. Emotional and Behavioral Disorders in Youth, 4(4), 83-105.

Mihalopoulos, C., Harris, M., Henry, L., Harrigan, S., \& McGorry, P. (2009). Is early intervention in psychosis cost-effective over the long term? Schizophrenia bulletin, 35(5), 909-918. DOI:10.1093/schbul/sbp054

Miller, N.E. \& Dollard, J. (1941). Social learning and imitation. New Haven: Yale University Press.

Mishler. G.E. (1991). Research Interviewing. Context and Narrative. Harvard University Press.

Morse, J. M. (1991). Approaches to qualitative-quantitative methodological triangulation. Nursing Research, 40(2), 120-123.

Morse, J. M. (1999). Myth \#93: Reliability and validity are not relevant to qualitative inquiry. Qualitative health research, 9(6), 717-718.

Mulaik, S. A. (2005). Looking back on the indeterminacy controversies in factor analysis. Contemporary Psychometrics: A Festschift for Roderick P. McDonald, 173-206.

Mullen, E. J., \& Bacon, W. (2004). A Survey of Psychiatrists, Psychologists, and Social. Evidence-based practice manual: Research and outcome measures in health and human services, 210.

National Assembly for Wales (2009/2010) NHS Wales: Annual Operating Framework 2009/2010. Retrieved September 122016 from NHS Wales website, http://www.wales.nhs.uk/document/175974.

National Institute for Clinical Excellence. (2009). Schizophrenia Core Interventions in the Treatment and Management of Schizophrenia in Primary and Secondary care. Guideline 82. London. NICE. Retrieved May 122017 from NICE website, http://guidance.nice.org.uk/CG82. 
National Institute for Clinical Excellence. (2013). Psychosis and Schizophrenia in Children and Young People. Guideline 155. London. NICE. Retrieved June 292017 from NICE website, https://www.nice.org.uk/guidance/cg155.

National Institute for Clinical Excellence. (2014). Psychosis and Schizophrenia in Adults: Prevention and Management. Guideline 178. London. NICE.

Retrieved December 152018 from NICE website, https://www.nice.org.uk/guidance/cg178.

Nelson, T. D., Steele, R. G., \& Mize, J. A. (2006). Practitioner attitudes toward evidence-based practice: Themes and challenges. Administration and Policy in Mental Health and Mental Health Services Research, 33(3), 398-409.

NHS Confederation. (2011). Early Intervention Briefing. Mental Health NHS Confederation. Issue 11. Retrieved July 62017 from IRIS Initiative website, http://www.iris-initiative.org.uk/silo/files/nhs-confederation-briefing-on-earlyintervention-in-psychosis.pdf.

Nilsen, P. (2015). Making sense of implementation theories, models and frameworks. Implementation science, 10(1), 53.

Nordentoft, M., Melau, M., Iversen, T., Petersen, L., Jeppesen, P., Thorup, A., ... \& Jørgensen, P. (2015). From research to practice: how OPUS treatment was accepted and implemented throughout Denmark. Early intervention in psychiatry, 9(2), 156-162.

Nordentoft, M., Rasmussen, J. Ø., Melau, M., Hjorthøj, C. R., \& Thorup, A. A. (2014). How successful are first episode programs? A review of the evidence for specialized assertive early intervention. Current opinion in psychiatry, 27(3), 167-172.

Norman, R. M., Lewis, S. W., \& Marshall, M. (2005). Duration of untreated psychosis and its relationship to clinical outcome. The British journal of psychiatry, 187(48), s19-s23.

Novick, G. (2008). Is there a bias against telephone interviews in qualitative research?. Research in nursing \& health, 31(4), 391-398.

Nursing and Midwifery Council (NMC), 2015. The code: professional standards of practice and behaviour for nurses and midwives. London: NMC.

https://www.nmc.org.uk/globalassets/sitedocuments/nmc-publications/nmccode.pdf

Nutley , S M , Powell , A E \& Davies , H. T. O 2013 , What counts as good evidence. Alliance for Useful Evidence, London.

Nutley, S., \& Davies, H. T. (2000). Getting research into practice: making a reality of evidence-based practice: some lessons from the diffusion of innovations. Public money and management, 20(4), 35-42. 
Nutley, S., \& Davies, H. T. (2000). Getting research into practice: making a reality of evidence-based practice: some lessons from the diffusion of innovations. Public money and management, 20(4), 35-42.

Nutley, S., Walter, I., \& Davies, H. T. (2003). From knowing to doing: a framework for understanding the evidence-into-practice agenda. Evaluation, 9(2), 125-148.

Nutley, S.M. \& Davies, H.T.O. (1999b). Introducing evidence-based practice: the contrasting experiences of healthcare and probation service organisations in the UK', Public Policy and Administration, 14, (4), 39-57.

Nutley., S and Davies., H. (2000). Making a reality of evidence based practice. In Davies., T.O, Nutley., S and Smith P (Eds.), What works? Evidence-based policy and practice in public services ( $p$ 326). The Policy Press.

O'Cathain, A., Murphy, E., \& Nicholl, J. (2010). Three techniques for integrating data in mixed methods studies. Bmj, 341, c4587.

O'Cathain, A., Murphy, E., \& Nicholl, J. (2008). The quality of mixed methods studies in health services research. Journal of Health Services Research \& Policy, 13(2), 92-98.

Oestrich, I. H., Austin, S. F., \& Tarrier, N. (2007). Conducting research in everyday psychiatric settings: identifying the challenges to meaningful evaluation. Journal of Psychiatric and Mental Health Nursing, 14(1), 55-63.

Ogborne, A. C., Wild, T. C., Braun, K., \& Newton-Taylor, B. (1998). Measuring treatment process beliefs among staff of specialized addiction treatment services. Journal of Substance Abuse Treatment, 15(4), 301-312.

Ogilvie, D., Egan, M., Hamilton, V., \& Petticrew, M. (2005). Systematic reviews of health effects of social interventions: 2. Best available evidence: how low should you go?. Journal of Epidemiology \& Community Health, 59(10), 886-892.

Onwuegbuzie, A. J., and Leech, N. L. (2004). Enhancing the interpretation of "significant" findings: The role of mixed methods research. The Qualitative Report, 9, 770-792.

Pagoto, S. L., Spring, B., Coups, E. J., Mulvaney, S., Coutu, M. F., \& Ozakinci, G. (2007). Barriers and facilitators of evidence-based practice perceived by behavioral science health professionals. Journal of clinical psychology, 63(7), 695-705.

Panzano, P. \& Herman, L. (2005). Developing and sustaining evidence-based systems of mental health services. In Drake, R.E., Merrens, M.R. \& Lynde, D. E. (eds) Evidence based Mental Health Practice: A textbook. New York: Norton pp.243-272. 
Parmelli, E., Flodgren, G., Beyer, F., Baillie, N., Schaafsma, M. E., \& Eccles, M. $P$. (2011). The effectiveness of strategies to change organisational culture to improve healthcare performance: a systematic review. Implementation Science, 6(1), 33.

Patel, A., McCrone, P., Leese, M., Amaddeo, F., Tansella, M., Kilian, R., ... \& Knapp, M. (2013). Cost-effectiveness of adherence therapy versus health education for people with schizophrenia: randomised controlled trial in four European countries. Cost Effectiveness and Resource Allocation, 11(1), 12

Pawson, R., Boaz, A., Grayson, L., Long, A., \& Barnes, C. (2003). Types and quality of social care knowledge. Stage two: towards the quality assessment of social care knowledge. ESRC UK Center for Evidence Based Policy and Practice: Working Paper, 18.

Pelosi, A. J., \& Birchwood, M. (2003). Is early intervention for psychosis a waste of valuable resources?. The British Journal of Psychiatry, 182(3), 196-198.

Penn, D. L., Waldheter, E. J., Perkins, D. O., Mueser, K. T., \& Lieberman, J. A. (2005). Psychosocial treatment for first-episode psychosis: a research update. American journal of Psychiatry, 162(12), 2220-2220.

Perera, U., \& Taylor, M. (2014). NICE CG178 on psychosis-an evidence-based guideline. Prescriber, 25(9), 6-8.

Petersen, L., Jeppesen, P., Thorup, A., Abel, M. B., Øhlenschlæger, J., Christensen, T. $\varnothing ., \ldots \&$ Nordentoft, M. (2005). A randomised multicentre trial of integrated versus standard treatment for patients with a first episode of psychotic illness. Bmj, 331(7517), 602.

Pfeffer, J., \& Sutton, R. I. (2006). Evidence-based management. Harvard business review, 84(1), 62.

Pharoah, F., Mari, J. J., Rathbone, J., \& Wong, W. (2010). Family intervention for schizophrenia. Cochrane database of systematic reviews, (12). doi/10.1002/14651858.CD000088.pub3.

Pilling, S., Bebbington, P., Kuipers, E., Garety, P., Geddes, J., Orbach, G., \& Morgan, C. (2002). Psychological treatments in schizophrenia: I. Meta-analysis of family intervention and cognitive behaviour therapy. Psychological medicine, 32(5), 763-782.

Pilling, S., Bebbington, P., Kuipers, E., Garety, P., Geddes, J., Orbach, G., \& Morgan, C. (2002). Psychological treatments in schizophrenia: I. Meta-analysis of family intervention and cognitive behaviour therapy. Psychological medicine, 32(5), 763-782.

Pope, C., Mays, N., Ziebland, S., le May, A., Williams, S., Coombs, M., ... \& Ellis, J. (2000). Qualitative methods in health research. methods, 1, 2. 
Power, M. (1994) 'Introduction: from the science of accounts to the financial accountability of science' in accounting and science: Natural inquiry and commercial reason M Power (Ed.), 1-35 Cambridge: Cambridge University Press.

Presser, S., Couper, M. P., Lessler, J. T., Martin, E., Martin, J., Rothgeb, J. M., \& Singer, E. (2004). Methods for testing and evaluating survey questions. Public opinion quarterly, 68(1), 109-130.

Proctor, E. K., Knudsen, K. J., Fedoravicius, N., Hovmand, P., Rosen, A., \& Perron, B. (2007). Implementation of evidence-based practice in community behavioural health: Agency director perspectives. Administration and Policy in Mental Health and Mental Health Services Research, 34(5), 479-488.

Proctor, E. K., Landsverk, J., Aarons, G., Chambers, D., Glisson, C., \& Mittman, B. (2009). Implementation research in mental health services: An emerging science with conceptual, methodological, and training challenges. Administration and Policy in Mental Health and Mental Health Services Research, 36(1), 24-34

Proctor, E., Silmere, H., Raghavan, R., Hovmand, P., Aarons, G., Bunger, A., \& Hensley, M. (2011). Outcomes for implementation research: conceptual distinctions, measurement challenges, and research agenda. Administration and Policy in Mental Health and Mental Health Services Research, 38(2), 6576.

Prytys, M., Garety, P. A., Jolley, S., Onwumere, J., \& Craig, T. (2011). Implementing the NICE guideline for schizophrenia recommendations for psychological therapies: a qualitative analysis of the attitudes of CMHT staff. Clinical psychology \& psychotherapy, 18(1), 48-59.

Rabin, B. A., Brownson, R. C., Haire-Joshu, D., Kreuter, M. W., \& Weaver, N. L. (2008). A glossary for dissemination and implementation research in health. Journal of Public Health Management and Practice, 14(2), 117-123.

Rabin, B. A., Brownson, R. C., Haire-Joshu, D., Kreuter, M. W., \& Weaver, N. L. (2008). A glossary for dissemination and implementation research in

health. Journal of Public Health Management and Practice, 14(2), 117-123.

Radhakrishnan, M., McCrone, P., Lafortune, L., Everard, L., Fowler, D., Amos, T., ... \& Lavis, A. (2017). Cost-effectiveness of early intervention services for psychosis and fidelity to national policy implementation guidance. Early Intervention in Psychiatry

Raghavan, R., Bright, C. L., \& Shadoin, A. L. (2008). Toward a policy ecology of implementation of evidence-based practices in public mental health settings. Implementation Science, 3(1), 26.

Reichardt, C. S., \& Rallis, S.F. (1994). Qualitative and quantitative inquiries are not incompatible: A call for a new partnership. In C.S. Reichardt \& S.E. Rallis 
(Eds.), The qualitative-quantitative debate: New perspectives (pp.85-92). Thousand Oaks, CA:Sage.

Rentsch, J. R., \& Klimoski, R. J. (2001). Why do 'great minds' think alike?: Antecedents of team member schema agreement. Journal of Organizational Behavior, 22(2), 107-120.

Rethink Mental IIIness 2014. Lost generation: why young people with psychosis are being left behind and what needs to change, London, Rethink Mental Illness.

Porras, J.I. and Roberston, P.J. (1992) Organizational Development: Theory, Practice, Research. In: Dunnette, M.D. and Hough, L.M., Eds., Handbook of Industrial and Organizational Psychology, Consulting Psychologists Press, Palo Alto, 719-822.

Richardson, W. S., Wilson, M. C., Nishikawa, J., \& Hayward, R. S. (1995). The well-built clinical question: a key to evidence-based decisions. Acp j club, 123(3), A12-3.

Robinson, R. S., \& Driscoll, M. (1993). Qualitative research methods: an introduction. In M. R. Simonson, \& K. Abu-Omar, (Eds.), 15th annual proceedings of selected research and development presentations at the 1993 national convention of the Association for Educational Communications and Technology, New Orleans, LA. (pp. 833-844). Ames: Iowa State University

Rogers , E.M. (2003). Diffusion of Innovations ( $5^{\text {th }}$ ed.). New York, NY: Free Press

Rogers,E.M.(1995).Diffusion of innovations(4 ${ }^{\text {th }}$ ed.).New York: Free Press. Rosen, A. (2003). Evidence-based social work practice: Challenges and promise. Social Work Research, 27, 197-208.

Rosen, A., Proctor, E. K., Morrow-Howell, N., \& Staudt, M. (1995). Rationales for practice decisions: Variations in knowledge use by decision task and social work service. Research on Social Work Practice, 5, 501-523

Rosenheck, R. A. (2001). Organizational process: A missing link between research and practice. Psychiatric Services, 52(12), 1607-1612.

Rousseau, D. M. (1990). Assessing organizational culture: the case for multiple methods. In B. Schneider (Ed.), Organizational climate and culture (pp. 153192). San Francisco: Jossey-Bass.

Rousseau, D. M. (2005). Evidence-based management in health care. In C. Korunka \& P. Hoffmann (Eds.), Change and quality in human service work: 3346. Munich: Hampp.

Rousseau, D. M. (2006). Is there such a thing as "evidence-based management"? Academy of management review, 31(2), 256-269 
Rycroft-Malone, J., Seers, K., Titchen, A., Harvey, G., Kitson, A., \& McCormack, B. (2004). What counts as evidence in evidence-based practice?. Journal of advanced nursing, 47(1), 81-90.

Rye, M., Torres, E. M., Friborg, O., Skre, I., \& Aarons, G. A. (2017). The Evidence-based Practice Attitude Scale-36 (EBPAS-36): a brief and pragmatic measure of attitudes to evidence-based practice validated in US and Norwegian samples. Implementation Science, 12(1), 44.

Sackett, D.L., Richardson, W.S., Rosenberg, W. \& Hayes, R.B. (1997). Evidence-based Medicine: How to Practice and Teach EBM. New York: Churchill Livingstone.

Sackett, D. L., Rosenberg, W. M., Gray, J., Haynes, R. B., \& Richardson, W. S. (1996). Evidence Based Medicine: what it is and what it isn't. BMJ: British Medical Journal, 312(7023), 71-72.

Sackett, D. L., \& Rosenberg, W. M. (1995). The need for evidence-based medicine. Journal of the Royal Society of Medicine, 88(11), 620-624.

Sainsbury Centre for Mental Health (2003) The Economic and Social Costs of Mental IIIness, Accessed 11 April 2014.

http://www.centreformentalhealth.org.uk/pdfs/costs_of_mental_illness_policy_p aper_3.pdf.

Sarantakos, S., (2005). Social Research third edition. Palgrave Macmillan.

Scheirer, M. A. (2005). Is sustainability possible? A review and commentary on empirical studies of program sustainability. American Journal of Evaluation, 26(3), 320-347.

Schensul, S. L., Schensul, J. J., \& LeCompte, M. D. (1999). Essential ethnographic methods: Observations, interviews, and questionnaires (Vol. 2). Rowman Altamira.

Schoenwald, S. K., \& Hoagwood, K. (2001). Effectiveness, transportability, and dissemination of interventions: What matters when?. Psychiatric services, 52(9), 1190-1197.

Schoenwald, S. K., Letourneau, E. J., \& Halliday-Boykins, C. (2005). Predicting therapist adherence to a transported family-based treatment for youth. Journal of Clinical Child and Adolescent Psychology, 34(4), 658-670.

Shadish, W., Cook, T.D., \& Campbell, D.T. (2002). Experimental and quasiexperimental designs for general causal inference. Boston: Houghton Mifflin

Shaxson, $L$ (2005) 'Is your evidence robust enough? Questions for policy makers and practitioners' Evidence and Policy: A Journal of Research, Debate and Practice, Vol. 1, No. 1, pp.101-111. Stares. 
Shediac-Rizkallah, M.C., \& Bone, L. R. (1998). Planning for the sustainability of community based health programs: conceptual frameworks and future directions for research practice and policy. Health Education Research, 13 (1) 87-108.

Shiers D., Smith., J. (2010) Early Intervention in Psychosis. A briefing for service planners. IRIS. Policy into Practice National Mental Health Developing Unit. Retrieved April 102016 from, https://pdfs.semanticscholar.org/presentation/fdf2/f8dd566f6b69549f35cad7851 dd733b145f3.pdf.

Silverman, D. (2005). Doing qualitative research: second edition. London: Sage.

Simpson, A., Jones, J., Barlow, S., \& Cox, L. (2013). Adding SUGAR: service user and carer collaboration in mental health nursing research. Journal of psychosocial nursing and mental health services, 52(1), 22-30.

Singh, S. P. (2010). Early intervention in psychosis. The British Journal of Psychiatry, 196(5), 343-345.

Singh, S. P., \& Fisher, H. L. (2007). Early intervention services. Psychiatry, 6(8), 333-338.

Smircich, L., \& Stubbart, C. (1985). Strategic management in an enacted world. Academy of management Review, 10(4), 724-736.

Sober, E. (1981). The principle of parsimony. The British Journal for the Philosophy of Science, 32(2), 145-156.

Sparkes, A. C. (2001). Myth 94: Qualitative health researchers will agree about validity. Qualitative health research, 11(4), 538-552.

Spoth, R., Guyll, M., Redmond, C., Greenberg, M., \& Feinberg, M. (2011). Six-Year Sustainability of Evidence-Based Intervention Implementation Quality by Community-University Partnerships: The PROSPER Study. American journal of community psychology, 48(3-4), 412-425.

Stain, H. J., Mawn, L., Common, S., Pilton, M., \& Thompson, A. (2019). Research and practice for ultra-high risk for psychosis: A national survey of early intervention in psychosis services in England. Early intervention in psychiatry, 13(1), 47-52.

Stanley A.M. (2005). Parsimony/Occham's Razor. In B.S.Everitt \& D.C.Howell (Eds.), Encyclopedia of Statistics in Behavioural Science (pp. 1517-1518). Wiley.

Stewart, R. E., \& Chambless, D. L. (2007). Does psychotherapy research inform treatment decisions in private practice?. Journal of Clinical Psychology, 63(3), 267-281. 
Stirman, S. W., Kimberly, J., Cook, N., Calloway, A., Castro, F., \& Charns, M. (2012). The sustainability of new programs and innovations: a review of the empirical literature and recommendations for future research. Implementation Science, 7(1), 17.

Stokols, D., Misra, S., Moser, R. P., Hall, K. L., \& Taylor, B. K. (2008). The ecology of team science: understanding contextual influences on transdisciplinary collaboration. American journal of preventive medicine, 35(2), S96-S115.

Strang, D., \& Soule, S. A. (1998). Diffusion in organizations and social movements: From hybrid corn to poison pills. Annual review of sociology, 24(1), 265-290.

Strupp, H. H., \& Anderson, T. (1997). On the limitations of therapy manuals. Clinical Psychology: Science and Practice, 4(1), 76-82.

Sturges, J. E., \& Hanrahan, K. J. (2004). Comparing telephone and face-to-face qualitative interviewing: a research note. Qualitative research, 4(1), 107-118.

SurveyMonkey Inc. San Mateo, California USA. www.surveymonkey.com

Sutcliffe, S. and Court, J. 2005. Evidence-Based Policymaking: What is it? How does it work? What relevance for developing countries? Overseas Development Institute.

Swain, K., Whitley, R., McHugo, G. J., \& Drake, R. E. (2010). The sustainability of evidence-based practices in routine mental health agencies. Community mental health journal, 46(2), 119-129.

Sweeney, A., Beresford, P., Faulkner, A., Nettle, M and Rose, D. (2009). This is survivor research. Ross-on-Wye, PCCS Books.

Tait, L., Ryles, D., \& Sidwell, A. (2010). Strategies for engagement. Promoting recovery in early psychosis: A practice manual, 35-44.

Tashakkori, A., \& Creswell, J. W. (2007). The new era of mixed methods. Journal Mixed Methods Research, 1,3-7.

Tashakkori, A., \& Teddlie, C. (Eds.). (2009). Mixed methodology: combining the qualitative and quantitative approaches. Thousand Oaks, CA; Sage.

Tebes, J. K. (2005). Community science, philosophy of science, and the practice of research. American Journal of Community Psychology, 35(3-4), 213230.

Teddlie, C., \& Tashakkori, A. (2003). Major issues and controversies in the use of mixed methods in the social and behavioural sciences. In A. Tashakkori \& $\mathrm{C}$ 
Teddlie (Eds.), Handbook of mixed methods in social and behavioural research (pp.3-50) Thousand Oaks, CA: Sage.

The British Psychological Society (2009). Code of Ethics and Conduct.

Guidance Published by the Ethics Committee of the British Psychological Society. Retrieved June 152017 from the BPS website, https://beta.bps.org.uk/sites/beta.bps.org.uk/files/Policy\%20-

\%20Files/Code\%20of\%20Ethics\%20and\%20Conduct\%20\%282009\%29.pdf

The Schizophrenia Commission (2012). The abandoned illness. A Report by the Schizophrenia Commission. Rethink Mental illness. Retrieved:

https://www.rethink.org/about-us/the-schizophrenia-commission.

Titchen A. (2000) Professional Craft Knowledge in Patient-Centred Nursing and the Facilitation of Its Development. University of Oxford DPhil thesis. Ashdale Press, Kidlington.

Torrey, W. C., Lynde, D. W., \& Gorman, P. (2005). Promoting the implementation of practices that are supported by research: The National Implementing Evidence-Based Practice Project. Child and Adolescent Psychiatric Clinics, 14(2), 297-306.

University of Manchester (2013). The National Confidential Inquiry into Suicide and Homicide by people with mental illness. Annual Report: England, Northern Ireland, Scotland and Wales. Retrieved February 102016 from, University of Manchester website,

http://research.bmh.manchester.ac.uk/cmhs/research/centreforsuicidepreventio n/nci/reports/2016-report.pdf.

Verbeke, W., Volgering, M., \& Hessels, M. (1998). Exploring the conceptual expansion within the field of organizational behaviour: Organizational climate and organizational culture. Journal of Management Studies, 35(3), 303-329.

Verbeke, W., Volgering, M., \& Hessels, M. (1998). Exploring the conceptual expansion within the field of organizational behaviour: Organizational climate and organizational culture. Journal of Management Studies, 35(3), 303-329.

Von Bertalanffy, L. (1968). General system theory. New York, 41973(1968), 40.

Wales Audit Office (2005). Adult Mental Health Services in Wales: A Baseline Review of Service Provision. Wales Audit Office. Retrieved May 2007 from NHS website,

http://www.wales.nhs.uk/documents/adult_mental_health_services_baseline_re view.pdf.

Walshe, K., \& Rundall, T. G. (2001). Evidence-based management: from theory to practice in health care. Milbank Quarterly, 79(3), 429-457.

Weick, K. (1969). The social psychology of organizing (No. 04; HM131, W3.). 
Welsh Assembly Government (2018). Together for Mental Health Delivery Plan: 2016-2019. Progress Report October 2016-March 2018. Retrieved November 302018 from WAG website,

http://www.bavo.org.uk/user_resources/images/files/T4MH\%20full\%20deliverye n_(1).PDF.

Welsh Assembly Government. (2012). Policy Implementation Guidelines. Psychological Therapies in Wales. Retrieved October 2016 from WAG website, http://www.assembly.wales/Research\%20Documents/16-050-PsychologicalTherapies/16-050-Web-English.pdf.

Wenger, E., McDermott, R., \& Snyder, W. M. (2002). Seven principles for cultivating communities of practice. Cultivating Communities of Practice: a guide to managing knowledge, 4.

Willig, C. (1999). Beyond appearances: A critical realist approach to social constructionism. Social constructionist psychology: A critical analysis of theory and practice, 37-51.

Woltmann, E. M., Whitley, R., McHugo, G. J., Brunette, M., Torrey, W. C., Coots, L., ... \& Drake, R. E. (2008). The role of staff turnover in the implementation of evidence-based practices in mental health care. Psychiatric Services, 59(7), 732-737.

Zhang, M., Wang, M., Li, J., \& Phillips, M. R. (1994). Randomised-control trial of family intervention for 78 first-episode male schizophrenic patients. British Journal of Psychiatry, 165(suppl 24), 96-102.

Zokaei, A., Seddon, J., \& O'Donovan, B. (Eds.). (2010). Systems thinking: From heresy to practice: Public and private sector studies. Springer. 


\section{List of Appendices}

Appendix $1 \quad$ Search Strategy............................................................. 259

Appendix $2 \quad$ Demographics and EBPAS ............................................ 260

Appendix $3 \quad$ Assumptions of Normality............................................... 269

Appendix $4 \quad$ Semi-Structured Interview Schedule.................................. 271

Appendix $5 \quad$ Research Ethics Committee Letter (REC)......................... 279

Appendix $6 \quad$ National Research Ethics Service

Appendix $7 \quad$ Participant Consent........................................................ 281

Appendix 8 Quote, Code, Category, Theme and Theme Definition

Framework ....................................................... 286

Appendix $9 \quad$ Selection of Participants................................................. 297

Appendix $10 \quad$ All Recoding............................................................... 298

Appendix $11 \quad \begin{aligned} & \text { Summary of Demographic Variables Used in the } \\ & \text { Survey....................................................................... } 303\end{aligned}$

Appendix 12 Summary of Evidence Based Practice Attitudinal Scale

Appendix $13 \quad$ Regressions............................................................... 308

Appendix $14 \quad$ Models 1 to 4 Primary Discipline....................................... 314

Appendix 15 Mixed Methods Matrix.................................................. 319 


\section{Appendix 1: Search Strategy}

\section{Search 1}

implementation-of-change OR implement ${ }^{*}$ change OR change-management OR managing-change OR organisational-change OR organizational-change OR diffusion-of-innovation OR "dissemination and implementation"

\section{Search 2}

evidence-based OR evidence-informed OR research-based OR researchinformed OR EBP OR EBM OR clinical guidance OR clinical guidelines OR practice guidelines

\section{Search 3}

mental OR psychiatric

Search 4

S1 and S2 and S3

Databases searched: MEDLINE, CINAHL and PSYCINFO (all via the EBSCOPlatform).

Limits applied: English language, publication year Last 10 years 2007 to 2018. 
Appendix 2: Demographics and EBPAS

College of Human and Health Sciences

Coleg y Gwyddorau Dynol ac lechyd

\section{Demographics sheet}

Q1. Country

\begin{tabular}{|l|l|}
\hline England & $\square$ \\
\hline Wales & $\square$ \\
\hline
\end{tabular}

Q2. Health board or NHS Trust

Q3. Age

\begin{tabular}{|l|l|}
\hline 18-24 years & $\square$ \\
\hline 25-34 years & $\square$ \\
\hline $35-44$ years & $\square$ \\
\hline 45-54 years & $\square$ \\
\hline 55-64 years & $\square$ \\
\hline 65-74 years & $\square$ \\
\hline 75 years or older & $\square$ \\
\hline
\end{tabular}

Q4. Gender

\begin{tabular}{|l|l|}
\hline Male & $\square$ \\
\hline Female & $\square$ \\
\hline Prefer not to say & $\square$ \\
\hline $\begin{array}{l}\text { Other (please } \\
\text { specify) }\end{array}$ & $\square$ \\
\hline & \\
\hline
\end{tabular}


College of Human and Health Sciences

Coleg y Gwyddorau Dynol ac lechyd

Q5. Educational level (tick all that apply)

\begin{tabular}{|l|l|}
\hline $\begin{array}{l}\text { Professional } \\
\text { Qualification }\end{array}$ & $\square$ \\
\hline Degree & $\square$ \\
\hline Masters degree & $\square$ \\
\hline PhD & $\square$ \\
\hline MD & $\square$ \\
\hline Fellowship & $\square$ \\
\hline $\begin{array}{l}\text { Other (specify } \\
\text { below) }\end{array}$ & $\square$ \\
\hline & \\
\hline
\end{tabular}

\section{Q6. Primary discipline}

\begin{tabular}{|l|l|}
\hline Psychiatry & $\square$ \\
\hline Nursing & $\square$ \\
\hline Social Work & $\square$ \\
\hline $\begin{array}{l}\text { Occupational } \\
\text { therapy }\end{array}$ & $\square$ \\
\hline Psychology & $\square$ \\
\hline $\begin{array}{l}\text { Other (specify } \\
\text { below) }\end{array}$ & $\square$ \\
\hline &
\end{tabular}


College of Human and Health Sciences

Coleg y Gwyddorau Dynol ac lechyd

\section{Q7. Which of these options most closely reflects the model used in your service?}

(Please see definitions below)

\begin{tabular}{|l|l|}
\hline $\begin{array}{l}\text { Stand alone } \\
\text { specialist team } \\
\text { model }\end{array}$ & $\square$ \\
\hline Hub and Spoke & $\square$ \\
\hline $\begin{array}{l}\text { Enhanced } \\
\text { Community Mental } \\
\text { Health Team }\end{array}$ & $\square$ \\
\hline $\begin{array}{l}\text { Other (Please } \\
\text { specify below) }\end{array}$ & $\square$ \\
\hline & \\
\hline
\end{tabular}

\section{Definitions}

Several different service delivery models can be described as potential vehicles for delivering the key tasks and providing the key components of early intervention in psychosis. Such models include a traditional or enhanced Community Mental Health Team (CMHT) model (sometimes described as a 'dispersed' model), a Hub and Spoke model and a specialist team model. What follows below is a brief definition of each model.

Stand alone Specialist Service Model: This model involves staff being mainly or exclusively responsible for the care and treatment of people with early psychosis within a discrete, specialist, free standing team. Hub and Spoke Model: This model has been advocated and utilized in rural areas. It comprises specialist service staff that are embedded in local community mental health teams and supported by a central hub in terms of clinical leadership, supervision and training.

Enhanced Community Mental Health Team: Providing for the needs of young people with early psychosis and their families through a traditional CMHT. 


\section{Q8. Which types of services are provided by your service model?}

(Please see the definitions below)

\begin{tabular}{|l|l|}
\hline Inpatient & $\square$ \\
\hline Outpatient & $\square$ \\
\hline Day treatment & $\square$ \\
\hline Case management & $\square$ \\
\hline
\end{tabular}

\section{Definitions}

Inpatient: A patient who stays in a hospital receiving medical care or treatment.

Outpatient: A patient who attends a hospital for treatment without staying there overnight.

Day treatment: Partial hospitalisation is a type of service used to treat mental illness and substance abuse. In partial hospitalisation, the patient continues to reside at home, but commutes to a treatment centre up to seven days a week. Partial hospitalization focuses on the over treatment of the individual, and is intended to avert or reduce in-patient hospitalisation.

Case management: A course of action for a situation involving a individual and the implementation of such a program specifically the process by which all health-related matters of case and managed by a health professional.

\section{Q9. Does your organisation adhere to the following practice policies?}

\begin{tabular}{|l|l|}
\hline $\begin{array}{l}\text { The Mental Health } \\
\text { Policy } \\
\text { Implementation } \\
\text { Guideline DH } \\
\text { (2001) }\end{array}$ & $\square$ \\
\hline $\begin{array}{l}\text { NICE clinical } \\
\text { guideline on } \\
\text { psychosis and } \\
\text { schizophrenia } \\
\text { (2014) }\end{array}$ & \\
\hline $\begin{array}{l}\text { Welsh } \\
\text { Psychological } \\
\text { Therapies (2012) }\end{array}$ & \\
\hline $\begin{array}{l}\text { Mental Health } \\
\text { (Wales) Measure } \\
\text { (2010) }\end{array}$ & \\
\hline I don't know & \\
\hline $\begin{array}{l}\text { Other (Please } \\
\text { specify below) }\end{array}$ & $\square$ \\
\hline \multicolumn{2}{|l|}{} \\
\hline
\end{tabular}


Q10. Please indicate the primary service area for your organisation

(Please see the definitions below)

\begin{tabular}{|l|l|}
\hline Urban & $\square$ \\
\hline Rural & $\square$ \\
\hline Suburban & $\square$ \\
\hline
\end{tabular}

\section{Definitions:}

Urban: in, relating to, or characteristic of a town or city

Rural: in relating to, or characteristic of the countryside rather than town

Suburban: pertaining to, inhabiting, or being in a suburb or the suburbs of a city or town

Q11. How many years of experience do you have working in mental health services?

\begin{tabular}{|l|l|}
\hline $0-2$ & $\square$ \\
\hline $3-5$ & $\square$ \\
\hline $6-10$ & $\square$ \\
\hline $11-15$ & $\square$ \\
\hline 16 years or over & $\square$ \\
\hline $\begin{array}{l}\text { Other (specify } \\
\text { below) }\end{array}$ & $\square$ \\
\hline & \\
\hline
\end{tabular}

Q12. How long has your Early intervention team been established?

\begin{tabular}{|l|l|}
\hline 1 year & $\square$ \\
\hline 2 years & $\square$ \\
\hline 3 years & $\square$ \\
\hline 4 years & $\square$ \\
\hline 5 years & $\square$ \\
\hline 6 years & $\square$ \\
\hline 7 years & $\square$ \\
\hline 8 years & $\square$ \\
\hline 9 years & $\square$ \\
\hline 10 years & $\square$ \\
\hline 10 years or over & $\square$ \\
\hline
\end{tabular}


Q13. Can you tell me the number of care coordinators in your early intervention team?

Q14. Can you tell me the discipline of care coordinators in your team?

Q15. How familiar are you with the term "Evidence based practice" and "empirically supported treatments?" Please tick the box below. (Please see the definitions below)

\begin{tabular}{|l|l|}
\hline Not at all & $\square$ \\
\hline To a slight extent & $\square$ \\
\hline $\begin{array}{l}\text { To a moderate } \\
\text { extent }\end{array}$ & $\square$ \\
\hline To a great extent & $\square$ \\
\hline $\begin{array}{l}\text { To a very great } \\
\text { extent }\end{array}$ & $\square$ \\
\hline
\end{tabular}

\section{Definitions}

Evidence based practice (EBP): EBP is the integration of clinical expertise, patient values, and the best research evidence into the decision making process for patient care.

Empirically supported treatments (EST): ESTs are treatments that have medical evidence or research to show they work. 


\section{Evidence-Based Practice Attitude Scale (EBPAS)}

The following questions ask about your feelings about using new types of therapy, interventions, or treatments. Manualised therapy refers to any intervention that has specific guidelines and/or components that are outlined in a manual and/or that are to be followed in a structured/predetermined way. Fill in the boxes with a checked cross indicating the extent to which you agree with each item using the following scale:

\begin{tabular}{|c|c|c|c|c|c|c|c|c|}
\hline \multirow[t]{2}{*}{$\begin{array}{c}0 \\
\text { Not at all }\end{array}$} & \multirow[t]{2}{*}{$\begin{array}{l}1 \\
\text { To a slight } \\
\text { extent }\end{array}$} & \multirow{2}{*}{\multicolumn{2}{|c|}{$\begin{array}{c}2 \\
\text { To a } \\
\text { moderate } \\
\text { extent }\end{array}$}} & \multicolumn{4}{|c|}{$\begin{array}{c}4 \\
\text { To a very } \\
\text { great extent }\end{array}$} & \multirow[b]{2}{*}{4} \\
\hline & & & & 0 & 1 & 2 & 3 & \\
\hline \multicolumn{4}{|c|}{$\begin{array}{l}\text { 1. I like to use new types of therapy/interventions to help my } \\
\text { clients }\end{array}$} & & & 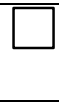 & $\square$ & \\
\hline \multicolumn{4}{|c|}{$\begin{array}{l}\text { 2. I am willing to try new types of therapy/interventions even if } \\
\text { I have to follow a treatment manual }\end{array}$} & $L$ & & & $\square$ & \\
\hline \multicolumn{4}{|c|}{$\begin{array}{l}\text { 3. I know better than academic researchers how to care for } \\
\text { my clients }\end{array}$} & L & & ] & $\square$ & \\
\hline \multicolumn{4}{|c|}{$\begin{array}{l}\text { 4. I am willing to use new and different types of } \\
\text { therapy/interventions developed by researchers }\end{array}$} & L & & 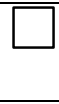 & $\square$ & \\
\hline \multicolumn{4}{|c|}{$\begin{array}{l}\text { 5. Research based treatments/interventions are not clinically } \\
\text { useful }\end{array}$} & L & & 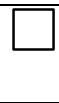 & 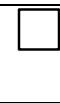 & \\
\hline \multicolumn{4}{|c|}{$\begin{array}{l}\text { 6. Clinical experience is more important than using manualised } \\
\text { therapy/treatment }\end{array}$} & L & & $\square$ & 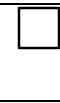 & \\
\hline \multicolumn{4}{|c|}{ 7. I would not use manualised therapy/interventions } & & & & & \\
\hline \multicolumn{4}{|c|}{$\begin{array}{l}\text { 8. I would try a new therapy/intervention even if it were very } \\
\text { different from what I am used to doing }\end{array}$} & & & & L & \\
\hline \multicolumn{4}{|c|}{$\begin{array}{l}\text { For questions 9-15: If you received training in a therapy or } \\
\text { intervention that was new to you, how likely would you be } \\
\text { to adopt it if: }\end{array}$} & & & & & \\
\hline \multicolumn{4}{|c|}{ 9. it was intuitively appealing? } & & & & & \\
\hline \multicolumn{4}{|c|}{ 10. it "made sense" to you? } & & & & & \\
\hline \multicolumn{4}{|c|}{ 11. it was required by your supervisor? } & & & & & \\
\hline \multicolumn{4}{|c|}{ 12. it was required by your organisation? } & & & & & \\
\hline \multicolumn{4}{|c|}{ 13. it was required by your country? } & & & & & \\
\hline \multicolumn{4}{|c|}{ 14. it was being used by colleagues who were happy with it? } & & & & & \\
\hline \multicolumn{4}{|c|}{ 15. you felt you had enough training to use it correctly? } & & & & & \\
\hline
\end{tabular}


If you are selected, would you take part in a semi-structured interview?

\begin{tabular}{|l|l|}
\hline $\begin{array}{l}\text { Yes I would be } \\
\text { willing to take part }\end{array}$ & $\square$ \\
\hline $\begin{array}{l}\text { No I wouldn't be } \\
\text { willing to take part }\end{array}$ & $\square$ \\
\hline
\end{tabular}

If you would like to be contacted again please enter your email address in the box below.

Thank you for completing the survey 
Appendix 3: Assumptions of Normality

A Bar chart showing assumption of normality for EBPAS score total

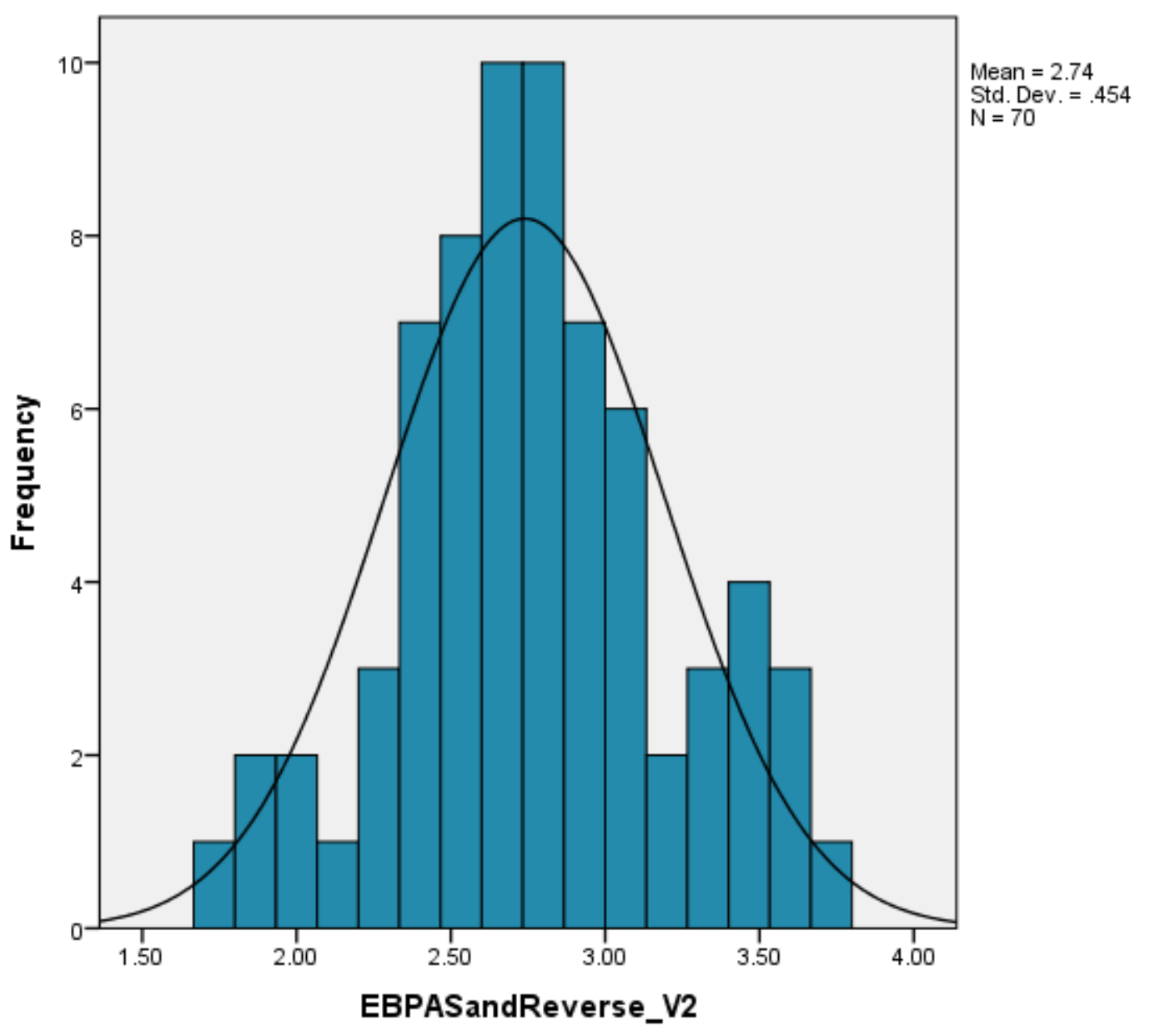


Appendix 3: Assumptions of Normality

A Bar chart showing assumption of normality for Requirements scale

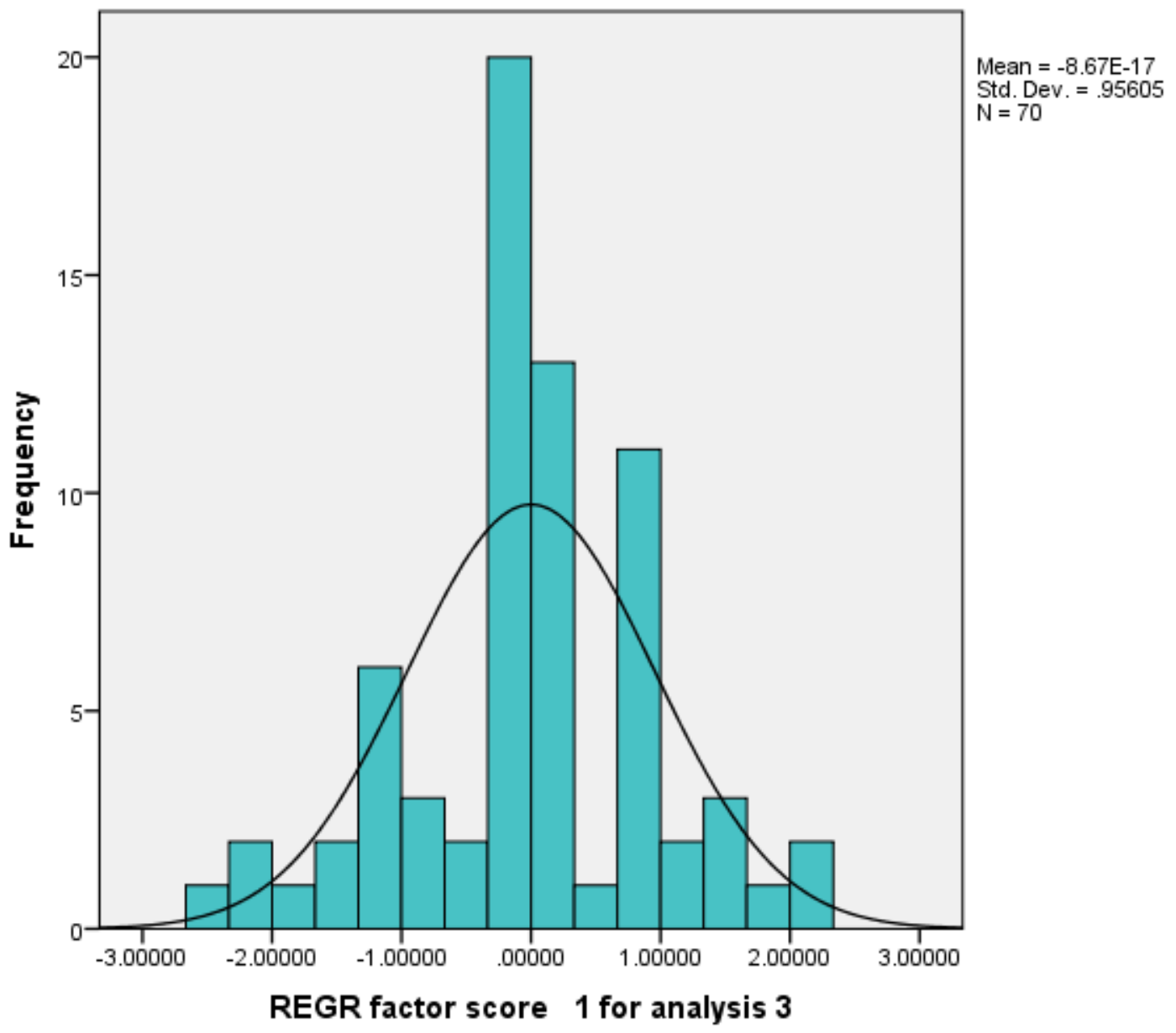


Appendix 3: Assumptions of Normality

A Bar chart showing assumption of normality for Openness scale

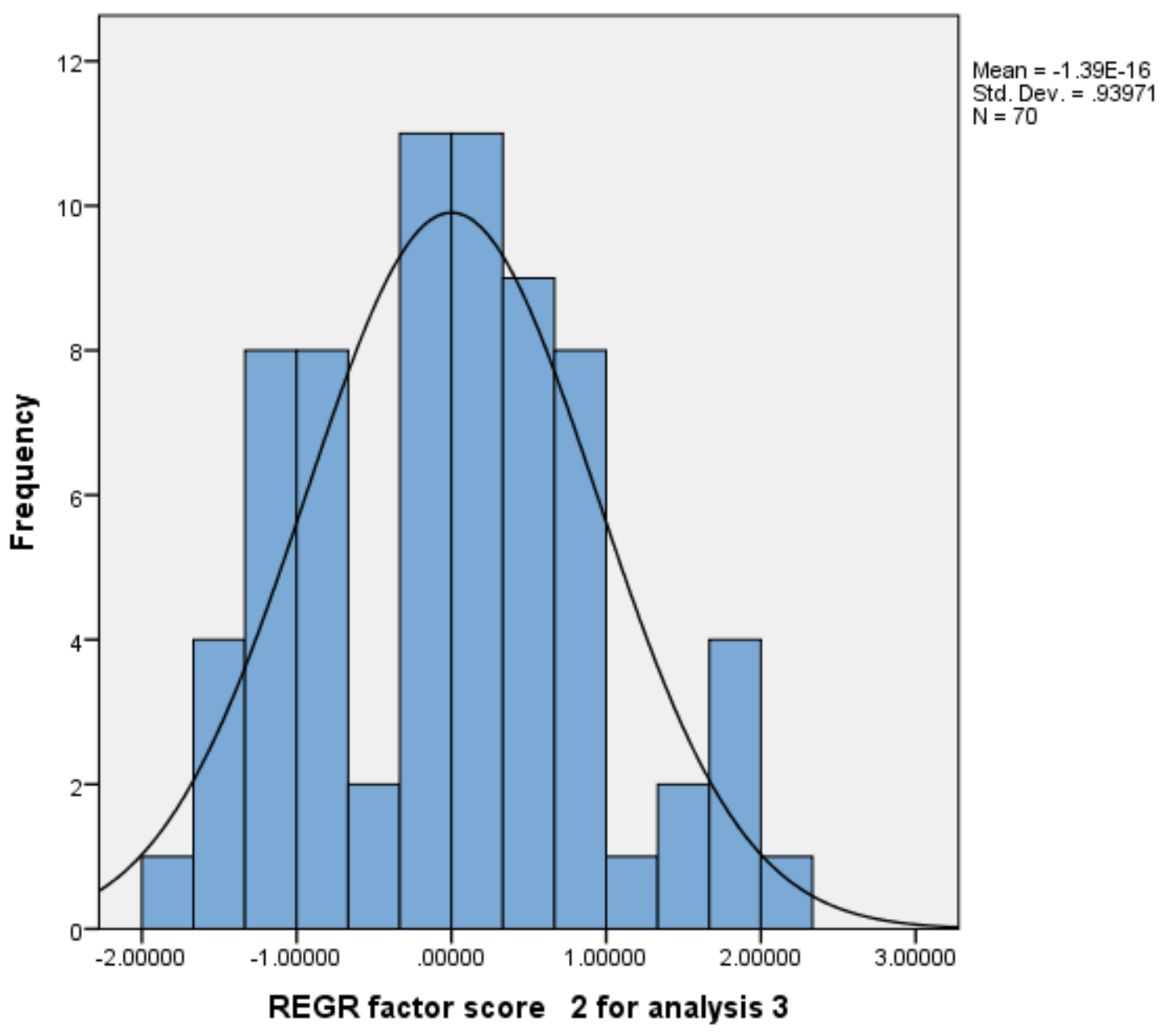




\section{Appendix 4: Semi-Structured Interview Schedule}

College of Human and Health Sciences

Coleg y Gwyddorau Dynol ac lechyd

\section{Semi-structured Interview Schedule}

Date of Interview:

Respondent's ID \#:

Country:

Health board/area:

Start time: Finish time:

\section{INTRODUCTION}

If you don't have any questions, let's begin. I am interested in every day experiences of implementation issues in your early intervention team, for example I am interested in issues relating to sustainability and fidelity.

The interview is organized into three sections and I will tell you when we move to a new section. I will read the questions exactly as they are written so that everyone is asked the same question. Section A, I will ask you about sustaining practices in your team, Section B, I will ask you about barriers and facilitators of Evidence Based Practices in your service and in Section C I will ask you about adherence to policies/protocols.

Feel free to ask me questions at any time if you are not sure what is wanted. The interview will take about 30 to 40 minutes to complete. 


\section{BACKGROUND INFORMATION AND TERMINONLOGY}

Regarding your role in your organization:

1. What is your job title?

2. When did you start this position (month/year)?

A couple of points on terminology:

First, this interview asks questions about "sustaining" practices in your team. What I mean is that your service is surviving, that your organisation continues to provide the services, your service continues to $273 \mathrm{nrol}$ new clients, and your service is funded. Once a service is closed down or discontinued, it is not sustained.

Fidelity refers to defined as the degree to which an intervention was implemented as it was prescribed in the original protocol or as it was intended by the program developers. 


\section{SECTION A}

\section{CRITICAL FACTORS}

What are three factors that you think have been critical in sustaining early intervention teams in your organization?

For example these could be factors at the country, NHS trust, or service level. (Note: if respondent gives a one word answer, prompt respondent by asking, "Can you say more?" If respondent stops after giving one or two factors, you can ask "Anything more?" If they say "no", then move onto the next section. (We can return to that question at the end if you wish so you can add anything else)

a)

Please tell me three factors that have worked against sustaining early intervention teams at your organization. (Note: if respondent gives a one-word answer, prompt respondent by asking, "Can you say more?" If respondent tops after giving one or two factors, you can ask "Anything more?" If they say "no",

then move on to the next section. (We can return to that question at the end if you wish so you can add anything else) 
College of Human and Health Sciences

Coleg y Gwyddorau Dynol ac lechyd

a)

b)

c)

Could you now tell me about anything else that will help me understand the sustaining (or not) of Evidence Based Practice at your organization? 
College of Human and Health Sciences

Coleg y Gwyddorau Dynol ac lechyd

Please tell me three things, either in or out of your control, that you think worked against sustaining Evidence Based Practice at your organisation.

That is, please tell me why you think your organisation is no longer offering Evidence Based Practice?
a)
b)
c) 


\section{SECTION B}

\section{BARRIERS AND FACILITATORS}

Please tell me what barriers your organisation faces with regards to implementing Evidence Based Practices in your service?

Prompts - Not always sure on how to link research with practice, Organizational change is difficult to accomplish, Research focus is inconsistent with clinical philosophy, No staff with statistical knowledge, Research results often not generalizable to our client population, lack of financing, Not enough information available to enable implementation, Conflicting research findings, No barriers.

Please tell me what facilitators your organisation faces with regards to implementing Evidence Based Practices in your service? Prompts adequate training, leadership, supervision, presence of a 'champion', adequate resources, and support for continuation of EBP at practice. 


\section{SECTION C}

\section{FIDELITY}

I am interested in hearing about fidelity in El teams, for my purposes fidelity is (defined as the degree to which an intervention was implemented as it was prescribed in the original protocol or as it was intended by the program developers). Can you tell me how you ensure fidelity in your team?

If participant doesn't mention adherence indicators then prompt with the following:-

\section{Note adherence indicators:}

Stand alone service model

Dedicated consultant psychiatrist input, full age range (14-35 yrs)

Care provided for up to three years

Assertive outreach worker

Extended opening hours

Case loads 10-15, adolescent provision

Primary care referral, designated assess to acute beds.

e.g. so tell me about caseload size and mix?

e.g. You haven't mentioned extended opening hours has this been an issue? 
If you haven't used fidelity assessment, can you please tell me why you haven't?

Is there anything you thought I would ask today and that we haven't covered?

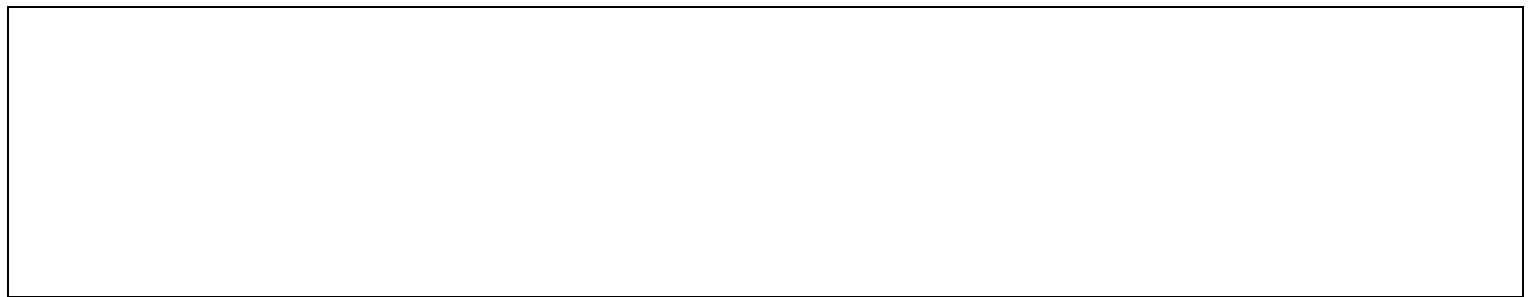

One final request: Is it OK if I email you with follow-up questions if I realize that I need clarification on anything? (Ask for participants email).

Participants email: 


\section{Appendix 5: Research Ethics Committee Letter}

February 18, 2015

Ref: 21014

Jo White

College of Medicine

Swansea University
Swansea University Prifysgol Abertawe

Dear Jo,

We would like to confirm that your research application entitled: 'Assessing implementation of evidence based practices in Early Intervention teams in Mental Health Services' was reviewed by members of the CHHS and CoM Research Ethics Committee on October 6, 2014 and was given approval on October 30, 2014.

Regards,

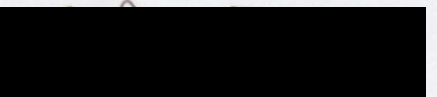

Professor Steve Edwards

Vice Chair, CHHS and College of Medicine Research Ethics Committee www.chhsresearchethics.swan.ac.uk

Pennoeth y Coleg - Coleg y Gumyddorau Dynol ac lechyd - Professor Melanie Jasper - Head of College • College of Human and Health Sciences Parc Singleton, Abertowe, SA2 8PP, Cymru, DU - Singleton Park, Swansea, SA2 8PP, Wales, UK Tel/Ffôn: +44 (0)1792 295789 Fax/Flacs: +44 (0)1792 295487 Web/Gwefan: www.swansea.ac.uk/humanondhealthsciences 


\section{Appendix 6: National Research Ethics Service (NRES)}

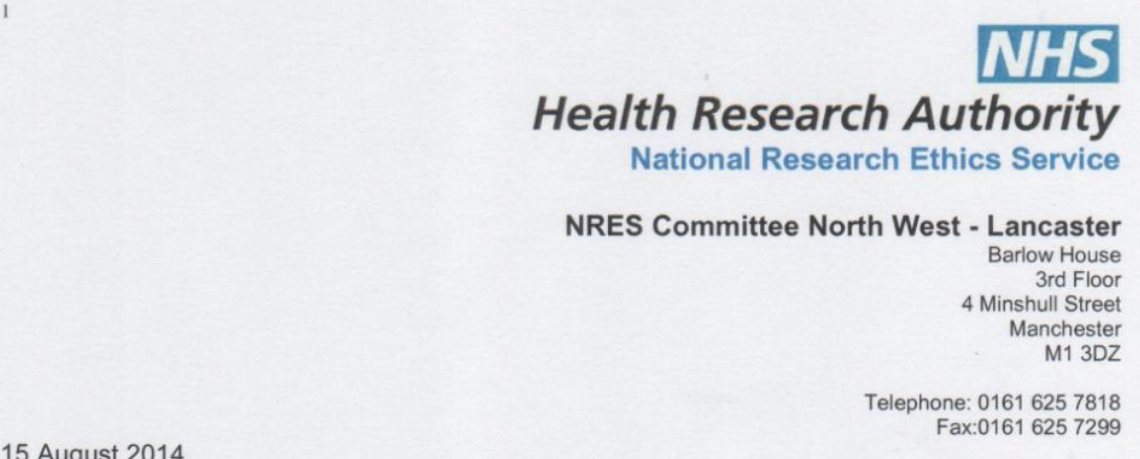

Ms Joanna White

Research Officer and PhD Student

Swansea University

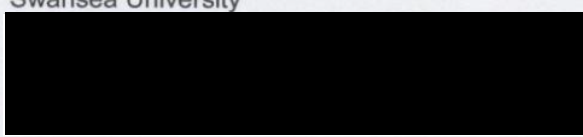

Dear Ms White

$\begin{array}{ll}\text { Study title: } & \begin{array}{l}\text { Assessing implementation of evidence based practices } \\ \text { in Early Intervention teams in Mental Health Services }\end{array} \\ \text { REC reference: } & 14 / N W / 1250 \\ \text { IRAS project ID: } & 118232\end{array}$

Thank you for your application for ethical review, which was received on 15 August 2014.

The application is not valid, for the following reason(s):

The form has not been electronically authorised.

From your application it would appear that your research is being conducted on NHS staff only and not on patients. If this is the case you do not need to get NHS REC approval and your University Ethics Committee can deal with this project. If, despite this, you still want NHS REC approval, you need to contact your local REC Regional Manager outlining your reasons for requiring NHS REC review and obtain permission for this.

You are welcome to book and submit another application through the Central Booking Service, taking into account the above points. The timeline for the ethical review will not commence until a valid application has been received.

Yours sincerely

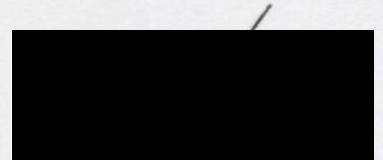

Mrs Carol Ebenezer

REC Manager

E-mail: nrescommittee.northwest-lancaster@nhs.net

Copy to:

Mr Ceri Jones, Ms Jemma Hughes, ABM University Health Board 
Appendix 7: Participant Consent

College of Human and Health Sciences

Coleg y Gwyddorau Dynol ac lechyd

\section{Assessing Implementation of Evidence Based Practices in Early Intervention teams in Mental Health Services.}

\section{Version 5 Date 08/10/14}

\section{Introduction}

I would like you to invite you to participate in this research on early intervention services that will form part of my PhD project.

You should only participate if you want to; choosing not to take part will not disadvantage you in anyway. Before you decide to take part it is important that you understand why the research is being done and what your participation will involve. Please take time to read the following information carefully and discuss it with others if you wish. If you do not understand anything please contact the researcher Jo White

or

\section{Background}

This study aims to examine attitudes and implementation to evidence based practices in a sample of lead practitioners in early intervention services including what they say about the organisational culture. The study will inform knowledge and understanding of evidence among healthcare managers, who are confronted with frequent service reconfiguration, policy changes and emerging evidence that may be conflicting in relation to provision of services. New knowledge originating from this research may prove useful for practitioners and organisations alike as they seek to plan and develop mental health services that are informed by evidence of effectiveness. 


\section{Aim of Project}

The project aims to develop an in-depth understanding of implementation of evidence based practices in early intervention mental health services in the UK. This will be achieved by;

- Examining attitudes and implementation of lead practitioners to evidence based practice in early intervention mental health services in the UK;

- In-depth interviews with a range of lead practitioners to examine every day experiences of implementation issues for example sustainability and fidelity.

\section{Why have I been invited to take part?}

You have been invited to take part because I am interested in assessing the implementation of evidence based practices in a sample of early intervention lead practitioners.

\section{Do I have to take part?}

No you don't have take part. If you did decide to take part you are still free to withdraw at any time and without giving a reason. A decision to withdraw at any time, or a decision not to take part, will have no effect on you, and no one else will know about this.

\section{What will happen to me if I take part?}

If you decide to take part, you will be given this information sheet to keep and be asked to sign a consent form. You will be invited to take part in a semistructured or telephone interview with the $\mathrm{PhD}$ researcher Jo White which will last 30 to 40 minutes at your work premises.

\section{Additional information}

I will not use your name in any way in reports of this work and it will not be known who took part. However, I might use some of the things you say in response to the questions to illustrate and support the findings of my $\mathrm{PhD}$ project. It is possible, but very unlikely, that someone who knows you very well might be able to identify you from such comments, but I will make every effort to minimise this possibility. 
The researcher will use a digital audio device to record the semi-structured interviews which will be analysed by the researcher and transcribed by using the guidance of Braun and Clarke (2006).

For practical purposes and to ensure timely completion a mixture of a professional transcription agency and the researcher will transcribe the audio recordings. All digital audio recordings will be downloaded onto the researchers PC for transcription and this will be password protected and uploaded via a secure portal to the professional transcription agencies server.

As soon as possible after the transcriptions have been received, they will be anonymised. Geographical locations will indicate only the trust or health board area.

All information is held anonymously, using ID codes, and kept in secure systems. Hard copies of the transcripts will be shredded at the end of the study and anonymous computer files held securely with a password protection for five years and then destroyed.

While I will do all I can to protect your confidentiality it should be noted that where instances of unsafe practices are revealed to the researcher, such reports can not be kept confidential and I am duty-bound to report these to the relevant authorities.

\section{Benefits}

Whilst there are no direct benefits for participants, it is hoped that they will find the experience interesting and rewarding.

\section{Further details}

For further details please contact Jo White, College of Medicine, Institute of Life Sciences 2 (ILS 2), Singleton Park, Swansea, SA2 8PP. Telephone:

Email: 


\section{SEMI STRUCTURED INTERVIEW/TELEPHONE INTERVIEW}

Please complete this form after you have read the information sheet (version 5, 08.10.14) and or listened to an explanation about the research.

Title of PhD Project: Assessing implementation of evidence based practices in Early Intervention teams in Mental Health Services.

Researcher Details: Jo White (PhD student), College of Medicine, Institute of Life Sciences 2 (ILS 2), Singleton Park, Swansea, SA2 8PP. Telephone:

Email:

Participant Identification Number:

1. I confirm that I have read and understood the information sheet (version 5). I have had the opportunity to consider the information, ask questions and have had these answered satisfactorily

2. I understand that my participation is voluntary and that I am free to withdraw at any time without giving a reason.

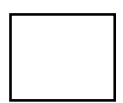

3. I agree to the use of anonymised quotes in publications.

4. I agree to the researcher making written notes during the semistructured interview.

5. I agree to the semi-structured interview being recorded using an audiorecording system by the researcher in order for a full transcript to be written up after the interview.

6 I understand that the audio recordings of the interviews will being sent to a third party for transcription prior to anonymisation.
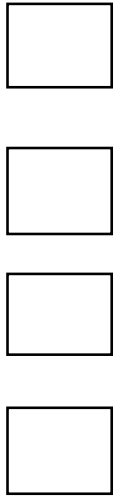

7. I agree to take part in the study.

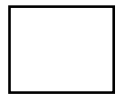




\section{Participant's Statement:}

I

agree that the PhD research project named above has been explained to me by my satisfaction and I agree to take part in the research project. I have read the information sheet about the project and I understand what the PhD project involves.

Signed.

Date.

Researcher's Statement:

I

confirm that I have carefully explained the nature, demands, and any foreseeable risks of the proposed research to the participant.

Signed.

Date 


\section{Appendix 8: Quote, Code, Category, Theme and Theme Definition Framework}

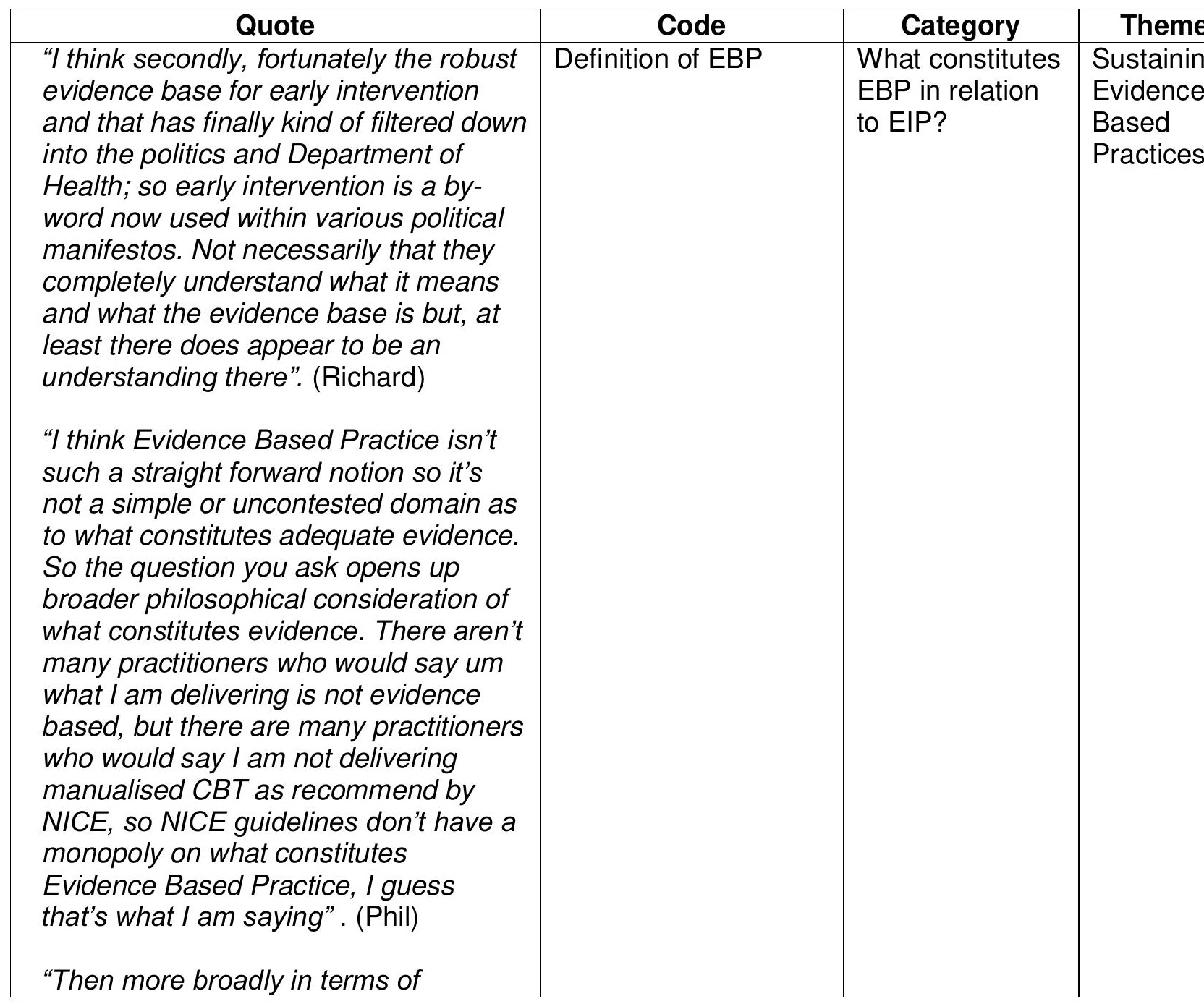




\begin{tabular}{|c|c|c|c|}
\hline $\begin{array}{l}\text { evidence-based practice I think also } \\
\text { one of the difficulties is the general } \\
\text { access of the work on the ground to an } \\
\text { ongoing discussion in the area about } \\
\text { what is evidence-based practice, what } \\
\text { is good evidence and what is not in } \\
\text { terms of the interventions that they are } \\
\text { using. Stuff like NICE guidelines } \\
\text { doesn't necessarily filter through to the } \\
\text { teams in a very systematic way of } \\
\text { making sure that all of the CMHT for } \\
\text { instance are regularly keeping up to } \\
\text { date with what is coming through from } \\
\text { NICE to make sure that what they are } \\
\text { doing is evidence-based practice". } \\
\text { (Steve) }\end{array}$ & & & \\
\hline $\begin{array}{l}\text { "Well I would say that the main one is } \\
\text { lack of understanding amongst the } \\
\text { managers who make more corporate or } \\
\text { clinically strategic decisions for the } \\
\text { trust. I don't think they generally have a } \\
\text { good enough understanding of day to } \\
\text { day practice in the trust but certainly } \\
\text { not specialist practice in the early } \\
\text { intervention teams. So they often send } \\
\text { out edicts that clearly compromise the } \\
\text { way we can and can't work". (Jeremy) } \\
\text { "I think there is quite a lack of } \\
\text { understanding of what we do. Um so } \\
\text { when lots of services are being } \\
\text { disbanded there was a quite a lot of } \\
\text { talk about, 'I don't know why they don't } \\
\text { get rid of EIP, I don't know what they }\end{array}$ & Lack of understanding & $\begin{array}{l}\text { Specialist } \\
\text { practice }\end{array}$ & $\begin{array}{l}\text { Sustainin } \\
\text { Evidence } \\
\text { Based } \\
\text { Practices }\end{array}$ \\
\hline
\end{tabular}


do'. You know they would have been better off working ....keeping assertive outreach. And getting rid of EIP and I think that's because what we do we do well. So the inpatient consultants don't see our patients cause we keep them out they don't know who we keep out. And um that we don't use up the crisis service. So I think you know there is a degree when doing things well that nobody knows what we doing because it doesn't impact on them". (Geraldine)

"I go back to the rationale for early intervention, and are people on board with it. In my experience, there's been a lot of staff, a lot of managers that don't agree that early intervention is needed, that felt that standard services were doing okay and why do we need this, why do we need to work differently. I found that as being quite a big issue. I don't think that people have a very clear understanding of what early intervention is about, exactly what we do and, more importantly, why we do it. That's been my experience". (Carolyn)

"The current financial economic climate, however you want to describe it, that's been a challenge for El (meaning EIP) services and l've seen this isn't the first early intervention service I've worked in I guess. I've 


\begin{tabular}{|c|c|c|c|}
\hline $\begin{array}{l}\text { seen that around the country, where } \\
\text { they've been disbanded or been gotten } \\
\text { rid of, they've just been absorbed back } \\
\text { into CMHTs". (Gareth) }\end{array}$ & & & \\
\hline $\begin{array}{l}\text { "I think there's also been a commitment } \\
\text { from some people working within the } \\
\text { health board to develop this sort of } \\
\text { service, you know, sort of, champion of } \\
\text { it". (Nigel) }\end{array}$ & Individual qualities & Champions & $\begin{array}{l}\text { Sustainin } \\
\text { Evidence } \\
\text { Based } \\
\text { Practices }\end{array}$ \\
\hline $\begin{array}{l}\text { "We've had good champions like the } \\
\text { [clinical psychologist] in Wales. He was } \\
\text { brilliant at maintaining enthusiasm for } \\
\text { the first episode network in spite of } \\
\text { getting all sorts of mixed messages } \\
\text { from senior management. He was very } \\
\text { good at maintaining optimism". (Steve) }\end{array}$ & & & \\
\hline $\begin{array}{l}\text { "Our clinical director was working with } \\
\text { us for well over a year in a clinical } \\
\text { capacity, within our trust - clinical } \\
\text { director is expected still to practice one } \\
\text { day a week. Ours is a psychologist by } \\
\text { background, she was working with our } \\
\text { service users and within our team. I } \\
\text { think that helped enormously for her to } \\
\text { understand what we do, and the value } \\
\text { of it and the demands and challenges". } \\
\text { (Martin) }\end{array}$ & Senior management & $\begin{array}{l}\text { Senior } \\
\text { management } \\
\text { (sub category) }\end{array}$ & $\begin{array}{l}\text { Sustainin } \\
\text { Evidence } \\
\text { Based } \\
\text { Practices }\end{array}$ \\
\hline $\begin{array}{l}\text { "One of the most important things for } \\
\text { us is having a clinical director who } \\
\text { works in early intervention and who } \\
\text { has been able to keep it on the radar". } \\
\text { (Richard) }\end{array}$ & & & \\
\hline
\end{tabular}




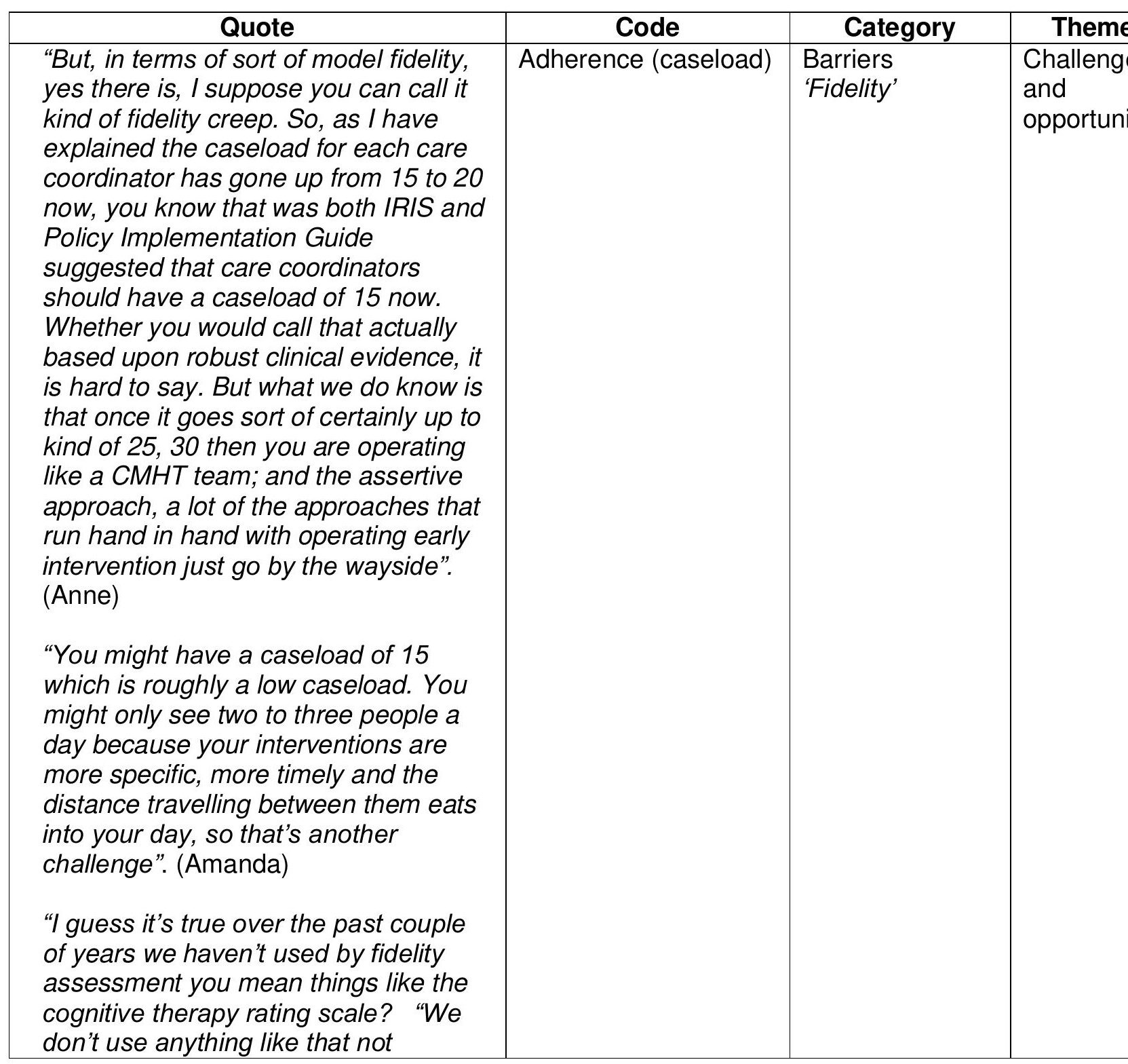


routinely anyway there may be occasions when people are doing particular courses that is used, but I would say there is a number there are two reasons obvious reasons that come to mind it's the resourcing question the kind of thing that takes extra time and ends up being neglected, but another thing I think real world clinical practice sometimes requires deviation from models because service users don't fit and sometimes the therapy doesn't fit as it might be prescribed in a manual. There's a requirement for flexibility in working which might actually mean that not that focusing on fidelity limits the flexibility for working that is required for this client group". (Phil)

"I have used them (tapes), but the reason why that's not ongoing, I think, is... There's a range of reasons, I think some people are more likely to take questions to supervision and focus it on that, rather than listening to tapes and scoring the tapes, so I think there's a cultural thing that makes that sometimes difficult, but also I think just the time that it takes to listen to a tape of a session and score it, given just the many challenges. Certain aspects of performance there are fidelity scales which can help, but the time taken to do them, score them, record them, and 


\begin{tabular}{|c|c|c|c|}
\hline $\begin{array}{l}\text { feed back is another factor that needs } \\
\text { to be made against the many } \\
\text { challenges that we have just to keep } \\
\text { the service going. Whilst that's a } \\
\text { facilitator of fidelity, I think it's with } \\
\text { increased challenges on time and } \\
\text { that's one of the first things that can } \\
\text { go". (Huw) }\end{array}$ & & & \\
\hline $\begin{array}{l}\text { "We had an awful lot of financial } \\
\text { pressures put on to us to establish an } \\
\text { early intervention service across the } \\
\text { six teams from what would have been } \\
\text { the equivalent of the strategic health } \\
\text { authority back in the day. So there was } \\
\text { some considerable political and } \\
\text { economic pressure to get it up and } \\
\text { running, but what's actually maintained } \\
\text { it or what's realised it I think has been } \\
\text { the calibre of the staff who were } \\
\text { initially recruited. I don't think there's } \\
\text { ever been any that have actually } \\
\text { threatened El (meaning ElP), but } \\
\text { they've made it more difficult. They are } \\
\text { principally the established services, } \\
\text { particularly established medical } \\
\text { services wanting to apply treatment as } \\
\text { usual to an early intervention } \\
\text { population. It doesn't work and might } \\
\text { be contra-indicated, so sort of standard } \\
\text { or high dose medications, inpatient } \\
\text { units that are designed for general } \\
\text { populations and not for first episode or } \\
\text { young people". (Jeremy) }\end{array}$ & Funding & $\begin{array}{l}\text { Barriers } \\
\text { 'Funding' }\end{array}$ & $\begin{array}{l}\text { Challeng } \\
\text { and } \\
\text { opportun }\end{array}$ \\
\hline "I suppose NHS cuts would be the & & & \\
\hline
\end{tabular}


other thing. Again, you have to invest in early intervention services to make those services long term. The current financial economic climate, however you want to describe it, that's been a challenge for El services(meaning EIP) and l've seen - this isn't the first early intervention service I've worked in I guess. I've seen that around the country, where they've been disbanded or been gotten rid of, they've just been absorbed back into CMHTs. The current economic climate, I would say, that's been definitely a challenge. If you look at the evidence, or the evidence that l've seen certainly, incidents of psychosis increase when the economic climate worsens. That's been a challenge. The other thing has been, this is quite Wales specific now, but a lack of doctors applying for training posts in Wales, which means that there's a shortage of staff grade level, registrar level staff, doctors. We've got a consultant, although his time his very precious. We only have him two days a week. Ideally we'd have a registrar or a staff grade level doctor but there aren't enough in the health board as a whole. That's one problem.

I would say we're probably a fairly low priority in terms of, or at least in the eyes of those people who allocate medical time to the different teams. 


\begin{tabular}{|c|c|c|c|}
\hline $\begin{array}{l}\text { The CMHTs are already struggling to } \\
\text { get enough doctors. We're not first on } \\
\text { the list to be allocated a registrar". } \\
\text { (Gareth) } \\
\text { "Obviously, like I said we haven't got } \\
\text { funding for a fully-fledged team at the } \\
\text { moment. This is just a pilot project, so } \\
\text { we haven't got separate funding or } \\
\text { anything else. Locally, there's limited } \\
\text { investment in this initiative. There's no } \\
\text { capital or recurring funds that are then } \\
\text { ring fenced." (Rob) }\end{array}$ & & & \\
\hline $\begin{array}{l}\text { "Yes, I guess one of the really } \\
\text { important things is supervision. } \\
\text { Supervision and availability of } \\
\text { supervision, both people having time to } \\
\text { take supervision and having people in } \\
\text { place to provide that, is really } \\
\text { important". (Huw) } \\
\text { "Yes. I mean, I think fidelity is an area } \\
\text { of difficulty. We've attempted to } \\
\text { address is by providing the team with } \\
\text { adequate resources, and also by } \\
\text { providing monthly supervision and } \\
\text { regular review of the, kind of, clinical } \\
\text { work with people". (Nigel) }\end{array}$ & Resources & $\begin{array}{l}\text { Barriers 'practice } \\
\text { proficiency' }\end{array}$ & $\begin{array}{l}\text { Challeng } \\
\text { and } \\
\text { opportun }\end{array}$ \\
\hline $\begin{array}{l}\text { "I think that there's only so much that } \\
\text { you can do in terms of changing a } \\
\text { culture as well, in terms of the } \\
\text { atmosphere and the climate within } \\
\text { which people work, and that if there } \\
\text { are strong personalities who are not } \\
\text { motivated by addressing those culture }\end{array}$ & Motivation & $\begin{array}{l}\text { Barrier } \\
\text { 'individual } \\
\text { attitude' }\end{array}$ & $\begin{array}{l}\text { Challeng } \\
\text { and } \\
\text { opportun }\end{array}$ \\
\hline
\end{tabular}




\begin{tabular}{|c|c|c|c|}
\hline $\begin{array}{l}\text { - those cultural elements, then that's } \\
\text { quite difficult to manage and it can lead } \\
\text { to disempowerment of staff and } \\
\text { reduced morale, which will have a very } \\
\text { negative impact on quality of care". } \\
\text { (Huw) } \\
\text { "Those things are totally out of our } \\
\text { control, but talking about evidence } \\
\text { based, I think that's down to the } \\
\text { individual. We can't really complain } \\
\text { that we haven't got the ideal } \\
\text { resources, because you can go into } \\
\text { the library and even if you have got } \\
\text { technophobia or whatever go through } \\
\text { anything, if you don't know how to go } \\
\text { about doing a lit search or whatever. } \\
\text { We've got very well equipped libraries } \\
\text { and so, yes, I don't think there's any } \\
\text { excuses really". (lan) }\end{array}$ & & & \\
\hline $\begin{array}{l}\text { "I don't know if it's - this is only my } \\
\text { perception, but I think particularly our } \\
\text { team, has excellent kind of } \\
\text { performance and quality data. We are } \\
\text { sort of consistently probably the best- } \\
\text { performing team in the trust, in terms } \\
\text { of meeting the key performance } \\
\text { indicators. We never sort of go into the } \\
\text { amber or red: this kind of thing". } \\
\text { (Martin) } \\
\text { "given increasing challenges in the } \\
\text { financial climate, the service has } \\
\text { become better at measuring the } \\
\text { outcomes, and measuring the }\end{array}$ & $\begin{array}{l}\text { Key performance } \\
\text { indicators, quality }\end{array}$ & $\begin{array}{l}\text { Protection of } \\
\text { early } \\
\text { intervention } \\
\text { services } \\
\text { 'outcome data' }\end{array}$ & $\begin{array}{l}\text { Challeng } \\
\text { and } \\
\text { opportun }\end{array}$ \\
\hline
\end{tabular}


performance of the service, and in communicating that data in a way which justifies the service and also hits certain targets in relation to funding. I think that if we're measuring client outcomes - working with people and collecting outcomes from them - is to use that data in a way which empowers both the client and the clinician so that it's used to support and develop quality, not as an indication of the performance of that staff member, because I think we'd get better quality and more realistic data when it's seen as a part of everyday practice that helps care, not to fit into a more target-based culture of performance. We'd get more data and better data. All of those things, I think, together would evidence and help sustain the quality of what we deliver". (Huw)

"In 2010, I think it was, we set up a database to try and capture information about first episode psychosis, our clients with first episode psychosis coming in to both the CMHTs, well coming into the service, so CMHTs, CAMHS and inpatient services". (Steve) 
Appendix 9: Selection of Participants

\begin{tabular}{|c|l|l|c|c|l|}
\hline $\begin{array}{c}\text { Participant } \\
\text { No }\end{array}$ & Eng/Wales & \multicolumn{1}{|c|}{ Gender } & Job & $\begin{array}{c}\text { EBPAS } \\
\text { Scores }\end{array}$ & Urbal \\
\hline 1 & England & Female & Psychiatrist & 3.47 & Urbar \\
\hline 2 & England & Male & Manager & 2.53 & Urbar \\
\hline 3 & England & Female & Psychiatrist & 1.80 & Subu \\
\hline 4 & England & Female & Manager & 2.67 & Subu \\
\hline 5 & England & Male & Psychologist & 2.60 & Rural \\
\hline 6 & England & Male & Psychiatrist & 2.20 & Rural \\
\hline 7 & England & Female & Psychiatrist & 2.33 & Urbar \\
\hline 8 & England & Male & Psychologist & 1.93 & Rural \\
\hline 9 & England & Male & Nurse & 3.00 & Subu \\
\hline 10 & Wales & Male & Nurse & 2.87 & Urbar \\
\hline 11 & Wales & Female & Therapist & 3.73 & Rural \\
\hline 12 & Wales & Female & Recovery practitioner & 3.40 & Urbar \\
\hline 13 & Wales & Male & Nurse & 3.27 & Urbar \\
\hline 14 & Wales & Male & Nurse & 2.33 & Urbar \\
\hline 15 & Wales & Male & Psychologist & 2.80 & Urbar \\
\hline & & & Associate specialist & & \\
16 & Wales & Male & psychiatry & 1.73 & Subur \\
\hline 17 & Wales & Male & Psychiatrist & 2.60 & rural \\
\hline 18 & Wales & Male & Nurse & 3.07 & Urbar \\
\hline
\end{tabular}


Appendix 10: Recoding

Table 1: Original variable - Country

\begin{tabular}{lcc}
\hline \multicolumn{1}{c}{ Country } & Frequency & Percent \\
\hline England & 53 & 75.7 \\
Wales & 17 & 24.3 \\
Total & 70 & 100 \\
\hline
\end{tabular}

Table 2: Original variable - age

\begin{tabular}{lcc}
\hline \multicolumn{1}{c}{ Age } & Frequency & Percent \\
\hline 18-24 years & 1 & 1.4 \\
25-34 years & 4 & 5.7 \\
35-44 years & 33 & 47.1 \\
45-54 years & 28 & 40.0 \\
55-64 years & 4 & 5.7 \\
Total & 70 & 100 \\
\hline
\end{tabular}

Table 2.1: New age variable recode

\begin{tabular}{lcc}
\hline \multicolumn{1}{c}{ Age } & Frequency & Percent \\
\hline 44 years or under & 38 & 54.3 \\
45 years or over & 32 & 45.7 \\
Total & 70 & 100 \\
\hline
\end{tabular}

Table 3: Original variable - Gender

\begin{tabular}{lcc}
\hline \multicolumn{1}{c}{ Gender } & Frequency & Percent \\
\hline Male & 33 & 47.1 \\
Female & 37 & 52.9 \\
Total & 70 & 100 \\
\hline
\end{tabular}


Table 4: Original variable - educational level

\begin{tabular}{lcc}
\hline \multicolumn{1}{c}{ Educational level } & Frequency & Percent \\
Professional Qualification & & \\
Yes & 27 & 38.6 \\
No & 43 & 61.4 \\
Total & 70 & 100 \\
Degree & & \\
Yes & 41 & 58.6 \\
No & 29 & 41.4 \\
Total & 70 & 100 \\
Masters degree & & \\
Yes & 27 & 38.6 \\
No & 43 & 61.4 \\
Total & 70 & 100 \\
PhD & & \\
Yes & 11 & 15.7 \\
No & 59 & 84.3 \\
Total & 70 & 100 \\
MD & & \\
Yes & 2 & 2.9 \\
No & 68 & 97.1 \\
Total & 70 & \\
Fellowship & & \\
Yes & 5 & 92.1 \\
No & 65 & 100 \\
Total & 70 & \\
\hline
\end{tabular}

Table 4.1: Highest level of education (four categories)

\begin{tabular}{|c|c|c|c|}
\hline Edt & cational level & Frequency & Percent \\
\hline Profession & qualification & 9 & 12.9 \\
\hline Degree & & 24 & 34.3 \\
\hline Masters & & 20 & 28.6 \\
\hline $\mathrm{PhD}$ & & 10 & 14.3 \\
\hline MD & recoded to Dr/fellow & 2 & 2.9 \\
\hline Fellowship & & 5 & 7.1 \\
\hline Total & & 70 & 100 \\
\hline
\end{tabular}

Table 4.2: Recode for educational level four categories

\begin{tabular}{lcc}
\hline \multicolumn{1}{c}{ Educational level } & Frequency & Percent \\
\hline Professional & 9 & 12.9 \\
qualification & & \\
Degree & 24 & 34.3 \\
Masters & 20 & 28.6 \\
Dr or Fellow & 17 & 24.3 \\
Total & 70 & 100 \\
\hline
\end{tabular}


Table 5: Original variable - primary discipline

\begin{tabular}{lcc}
\hline \multicolumn{1}{c}{ Primary discipline } & Frequency & Percent \\
\hline Psychiatry & 12 & 17.1 \\
Nursing & 35 & 50.0 \\
Social Work & 3 & 4.3 \\
Occupational Therapy & 4 & 5.7 \\
Psychology & 13 & 18.6 \\
Other & 2 & 2.9 \\
Missing & 1 & 1.4 \\
Total & 70 & 100 \\
\hline
\end{tabular}

Table 5.1: Recode primary discipline

\begin{tabular}{lcc}
\hline \multicolumn{1}{c}{ Primary discipline } & Frequency & Percent \\
\hline Nursing & 35 & 50.0 \\
Psychology & 13 & 18.6 \\
Psychiatry & 12 & 17.1 \\
Therapists / Social & 10 & 14.3 \\
work & & \\
Total & 70 & 100 \\
\hline
\end{tabular}

Table 6: Original variable - service model

\begin{tabular}{lcc}
\hline \multicolumn{1}{c}{ Service model } & Frequency & Percent \\
\hline $\begin{array}{l}\text { Stand alone specialist team } \\
\text { model }\end{array}$ & 47 & 67.1 \\
Hub and Spoke & 18 & 25.7 \\
$\begin{array}{l}\text { Enhanced Community } \\
\text { Mental Health Team }\end{array}$ & 5 & 7.1 \\
Total & 70 & 100 \\
\hline
\end{tabular}

Table 7: Original variable - service model

\begin{tabular}{lcc}
\hline \multicolumn{1}{c}{ Service model } & Frequency & Percent \\
\hline Inpatient & 13 & \\
Yes & 57 & 18.6 \\
No & 70 & 81.4 \\
Total & & 100 \\
Outpatient & 21 & \\
Yes & 49 & 30.0 \\
No & 70 & 70.0 \\
Total & & 100 \\
Case Management & 66 & \\
Yes & 4 & 94.3 \\
No & 70 & 5.7 \\
Total & & 100 \\
Day Treatment & 10 & \\
Yes & 60 & 14.3 \\
No & 70 & 85.7 \\
Total & & 100 \\
\hline
\end{tabular}


Table 8: Original variable - adherence to policy guidelines

\begin{tabular}{lcc}
\hline \multicolumn{1}{c}{ Adherence to policies } & Frequency & Percent \\
\hline The Mental Health Policy & & \\
Implementation Guidelines DH & & \\
(2001) & 55 & 78.6 \\
Yes & 15 & 21.4 \\
No & 70 & 100 \\
Total & & \\
NICE clinical guideline on & & \\
psychosis and schizophrenia & & \\
(2014) & 57 & 81.4 \\
Yes & 13 & 18.4 \\
No & 70 & 100 \\
Total & & \\
Welsh Psychological Therapies & 7 & 10.0 \\
Yes & 63 & 90.0 \\
No & 70 & 100 \\
Total & & \\
Mental Health (Wales) Measure & & \\
(2010) & 16 & 22.9 \\
Yes & 54 & 77.1 \\
No & 70 & 100 \\
Total & & \\
\hline
\end{tabular}

Table 9: Original variable for primary service area

\begin{tabular}{lcc}
\hline \multicolumn{1}{c}{ Primary service area } & Frequency & Percent \\
\hline Urban & 43 & 61.4 \\
Rural & 17 & 24.3 \\
Suburban & 10 & 14.3 \\
Total & 70 & 100 \\
\hline
\end{tabular}

Table 10: Original variable - years of experience working in mental health services

\begin{tabular}{lcc}
\hline $\begin{array}{c}\text { Years of experience in } \mathrm{MH} \\
\text { services }\end{array}$ & Frequency & Percent \\
\hline $0-2$ years & 1 & 1.4 \\
$3-5$ years & 1 & 1.4 \\
$6-10$ years & 8 & 11.4 \\
$11-15$ years & 20 & 28.6 \\
16 years or over & 40 & 57.1 \\
Total & 70 & 100 \\
\hline
\end{tabular}


Table 10.1: Recode frequency for years of experience

\begin{tabular}{lcc}
\hline $\begin{array}{c}\text { Years of experience } \\
\text { recoded }\end{array}$ & Frequency & Percent \\
\hline $0-10$ years & 10 & 14.3 \\
$11-15$ years & 20 & 28.6 \\
16 years or over & 40 & 57.1 \\
Total & 70 & 100 \\
\hline
\end{tabular}

Table 11: Original variable: Early Intervention team been established

\begin{tabular}{|c|c|c|c|}
\hline \multicolumn{2}{|c|}{$\begin{array}{l}\text { How long has El team been } \\
\text { established }\end{array}$} & Frequency & Percent \\
\hline 1 year & & 9 & 12.9 \\
\hline 2 years & & 0 & 0 \\
\hline 3 years & \} recode 1 & 1 & 1.4 \\
\hline 4 years & & 3 & 4.3 \\
\hline 5 years & & 2 & 2.9 \\
\hline 6 years & & 7 & 10.0 \\
\hline 7 years & recode 2 & 4 & 5.7 \\
\hline 8 years & & 5 & 7.1 \\
\hline 9 years & & 3 & 4.3 \\
\hline 10 years & Yecode 3 & 17 & 24.3 \\
\hline 11 years o & over \}recode 4 & 19 & 27.1 \\
\hline
\end{tabular}

Table 11.1: Recode frequency for duration of Early Intervention team been established

\begin{tabular}{lcc}
\hline \multicolumn{1}{c}{$\begin{array}{c}\text { Duration of El (Four } \\
\text { categories) }\end{array}$} & Frequency & Percent \\
\hline $1-5$ years & 15 & 21.4 \\
$6-9$ years & 19 & 27.1 \\
10 years & 17 & 24.3 \\
11 years or over & 19 & 27.1 \\
Total & 70 & 100 \\
\hline
\end{tabular}


Appendix 11: Summary of demographic variables used in the survey

\begin{tabular}{|c|c|c|}
\hline Variable & Frequency & $\%$ \\
\hline $\begin{array}{c}\text { Country } \\
-\quad \text { England } \\
-\quad \text { Wales }\end{array}$ & $\begin{array}{l}53 \\
17 \\
\end{array}$ & $\begin{array}{l}75.7 \\
24.3\end{array}$ \\
\hline $\begin{aligned} \text { Age } & \\
- & 44 \text { years or under } \\
- & 45 \text { years or over }\end{aligned}$ & $\begin{array}{l}38 \\
32\end{array}$ & $\begin{array}{l}54.3 \\
45.7\end{array}$ \\
\hline $\begin{aligned} \text { Gender } & \\
- & \text { Male } \\
- & \text { Female }\end{aligned}$ & $\begin{array}{l}33 \\
37\end{array}$ & $\begin{array}{l}47.1 \\
52.9\end{array}$ \\
\hline $\begin{array}{cl}\text { Educational level } \\
\text { - } & \text { Professional qualification } \\
\text { - } & \text { Degree } \\
\text { - } & \text { Masters } \\
\text { - } & \text { Dr or fellow }\end{array}$ & $\begin{array}{c}9 \\
24 \\
20 \\
17\end{array}$ & $\begin{array}{l}12.9 \\
34.3 \\
28.6 \\
24.3\end{array}$ \\
\hline $\begin{aligned} & \text { Primary discipline } \\
& \text { - } \text { Nursing } \\
& \text { - } \text { Psychology } \\
& \text { - } \text { Psychiatry } \\
& \text { - } \text { Therapists / Social work }\end{aligned}$ & $\begin{array}{l}35 \\
13 \\
12 \\
10\end{array}$ & $\begin{array}{l}50.0 \\
18.6 \\
17.1 \\
14.3\end{array}$ \\
\hline 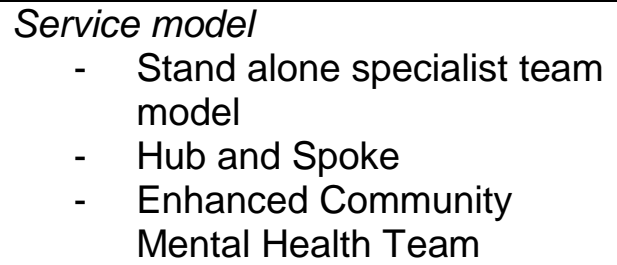 & $\begin{array}{c}47 \\
\\
18 \\
5\end{array}$ & $\begin{array}{c}67.1 \\
25.7 \\
7.1\end{array}$ \\
\hline 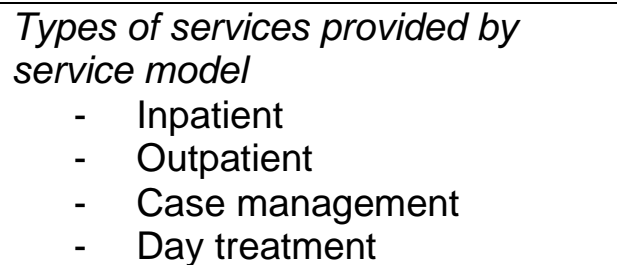 & $\begin{array}{l}13 \\
21 \\
66 \\
10\end{array}$ & $\begin{array}{l}18.6 \\
30.0 \\
94.3 \\
14.3\end{array}$ \\
\hline $\begin{array}{c}\text { Adherence to practice policies } \\
\text { - } \quad \text { The Mental Health Policy } \\
\text { Implementation Guidelines } \\
\text { DH (2001) } \\
\text { - } \quad \text { NICE clinical guideline on } \\
\text { psychosis and } \\
\text { schizophrenia (2014) } \\
\text { - Welsh Psychological } \\
\text { Therapies } \\
\text { - Mental Health (Wales) } \\
\text { Measure }\end{array}$ & $\begin{array}{c}7 \\
16\end{array}$ & $\begin{array}{l}10.0 \\
22.9\end{array}$ \\
\hline
\end{tabular}




\begin{tabular}{|c|c|c|}
\hline \multicolumn{1}{|c|}{ Variable } & Frequency & $\%$ \\
\hline Primary service area & & \\
- Urban & 43 & 61.4 \\
- Rural & 17 & 24.3 \\
- Suburban & & 14.3 \\
\hline Years experience of working in MH & & \\
services & 10 & 14.3 \\
- 0-10 years & 20 & 28.6 \\
- 11-15 years & 40 & 57.1 \\
- 16 years or over & & \\
\hline How long has El team been & 15 & 21.4 \\
established & 19 & 27.1 \\
- 1-5 years & 17 & 24.3 \\
- 6-9 years & 19 & 27.1 \\
- 10 years & & \\
\hline
\end{tabular}




\section{Appendix 12 Summary of Evidence Based Practice Attitudinal Scale measure}

\begin{tabular}{lcc}
\hline \multicolumn{1}{c}{ Scale } & Frequency & $\%$ \\
\hline Requirements & & \\
Required by supervisor & & \\
Not at all & 4 & 5.7 \\
To a slight extent & 11 & 15.7 \\
To a moderate extent & 35 & 50.0 \\
To a great extent & 15 & 21.4 \\
To a very great extent & 5 & 7.1 \\
Mean & 2.09 & \\
SD & 0.94 &
\end{tabular}

Required by organisation

Not at all

To a slight extent

To a moderate extent

To a great extent

$20 \quad 28.6$

To a very great extent

4

5.7

Mean

2.10

SD

Required by country

Not at all

To a slight extent

To a moderate extent

To a great extent

To a very great extent

Mean

0.0

7.1

SD

$\begin{array}{cc}6 & 8.6 \\ 12 & 17.1 \\ 29 & 41.4 \\ 17 & 24.3 \\ 6 & 8.6 \\ 2.07 & \\ 1.05 & \end{array}$

\section{Appeal}

Intuitively appealing

Not at all

To a slight extent

To a moderate extent

To a great extent

To a very great extent

Mean

SD

\section{4}

$\begin{array}{cc}4 & 5.7 \\ 17 & 24.3\end{array}$

$35 \quad 50.0$

$13 \quad 18.6$

Made sense to you

Not at all

2.79

0.86

To a slight extent

To a moderate extent

To a great extent

$\begin{array}{ll}13 & 18.6 \\ 30 & 42.9\end{array}$

To a very great extent

Mean

SD

Happy with it

Not at all

To a slight extent

$1 \quad 1.4$

$1 \quad 1.4$

To a moderate extent

To a great extent 
To a very great extent

Mean

SD

Enough training to use it correctly

Not at all

To a slight extent

To a moderate extent

To a great extent

To a very great extent

Mean

SD

\section{Openness}

Help my clients

Not at all

To a slight extent

To a moderate extent

To a great extent

To a very great extent

Mean

SD

Treatment manual

Not at all

To a slight extent

To a moderate extent

To a great extent

To a very great extent

Mean

SD

Developed by researchers

Not at all

To a slight extent

To a moderate extent

To a great extent

To a very great extent

Mean

SD

Would try therapy/interventions different than

usual

Not at all

To a slight extent

To a moderate extent

To a great extent

To a very great extent

Mean

SD

\section{Divergence}

Know better than academic researchers

Not at all
8.6

2.67

0.71

\section{3}

4.3

4

5.7

$39 \quad 55.7$

$24 \quad 34.3$

3

4.3

3.20

0.73

3

4.3

$23 \quad 32.9$

$34 \quad 48.6$

$10 \quad 14.3$

3

4.3

2.73

0.76

11

15.7

23

32.9

25

35.7

11

15.7

11

15.7

2.51

0.94 


\begin{tabular}{|c|c|c|}
\hline To a slight extent & 22 & 31.4 \\
\hline To a moderate extent & 20 & 28.6 \\
\hline To a great extent & 4 & 5.7 \\
\hline To a very great extent & 3 & 4.3 \\
\hline Mean & 1.23 & \\
\hline SD & 1.07 & \\
\hline \multicolumn{3}{|c|}{$\begin{array}{l}\text { Research based treatments are not clinically } \\
\text { useful }\end{array}$} \\
\hline Not at all & 53 & 75.7 \\
\hline To a slight extent & 12 & 17.1 \\
\hline To a moderate extent & 5 & 7.1 \\
\hline To a great extent & 0 & 0 \\
\hline To a very great extent & 0 & 0 \\
\hline Mean & 0.31 & \\
\hline SD & 0.60 & \\
\hline \multicolumn{3}{|c|}{$\begin{array}{l}\text { Clinical experience is more important than using } \\
\text { manualised/therapy treatment }\end{array}$} \\
\hline Not at all & 9 & 12.9 \\
\hline To a slight extent & 20 & 28.6 \\
\hline To a moderate extent & 32 & 45.7 \\
\hline To a great extent & 9 & 12.9 \\
\hline To a very great extent & 0 & 0 \\
\hline Mean & 1.59 & \\
\hline SD & 0.87 & \\
\hline \multicolumn{3}{|c|}{ Would not use manualised therapy/interventions } \\
\hline Not at all & 55 & 78.6 \\
\hline To a slight extent & 10 & 14.3 \\
\hline To a moderate extent & 4 & 5.7 \\
\hline To a great extent & 0 & 0 \\
\hline To a very great extent & 1 & 1.4 \\
\hline Mean & 0.31 & \\
\hline SD & 0.71 & \\
\hline
\end{tabular}


Appendix 13: Single Linear Regression analysis Factor analysis 1 of 3 'Requirements

\begin{tabular}{|c|c|c|c|}
\hline & Variable & $\underline{B}$ & SE \\
\hline & Q3. Agerecode & $-0 . \overline{4} 12$ & $0 . \overline{226}$ \\
\hline & Q4. Newgender & 0.161 & 0.23 \\
\hline & Q5. Degree $(y / n)(B L)$ & & \\
\hline All together & Q5. Profqual (y/n) & -0.436 & 0.36 \\
\hline All together & Q5. Masters $(\mathrm{y} / \mathrm{n})$ & -0.051 & 0.27 \\
\hline All together & Q5. Drorfellow (yes/no) & 0.435 & 0.301 \\
\hline \multirow[t]{2}{*}{ Singular } & Q6.newprimarydiscipline & 0.089 & 0.072 \\
\hline & Q6. Nursing (BL) & & \\
\hline All together & Q6. Psychology (yes/no) & -0.192 & 0.307 \\
\hline All together & Q6. Psychiatry (yes/no) & -0.443 & 0.316 \\
\hline \multirow[t]{2}{*}{ All together } & Q6. Therapsw (yes/no) & 0.393 & 0.339 \\
\hline & Q7newstandalone (yes/no) (BL) & & \\
\hline All together & Q7newhubandspoke (yes/no) & -0.168 & 0.268 \\
\hline All together & Q7newenhancedcommunityMHT (yes/no) & -0.2 & 0.455 \\
\hline Singular & Q8.Inpatient (yes/no) & 0.112 & 0.296 \\
\hline Singular & Q8.Outpatient (yes/no) & 0.004 & 0.251 \\
\hline Singular & Q8.Casemanagement (yes/no) & 0.326 & 0.494 \\
\hline Singular & Q8.Daytreatment (yes/no) & 0.074 & 0.329 \\
\hline Singular & Q9.PracPolPIG (yes/no) & 0.716 & 0.267 \\
\hline Singular & Q9.PracPoINICE (yes/no) & 0.597 & 0.287 \\
\hline Singular & Q9.PracPolWelshPsychological (yes/no) & 0.759 & 0.372 \\
\hline \multirow[t]{2}{*}{ Singular } & Q9.PracPolMentalhealth (yes/no) & -0.154 & 0.273 \\
\hline & Q10newurban (Yes/no) (BL) & & \\
\hline All together & Q10newrural (Yes/no) & 0.274 & 0.272 \\
\hline All together & Q10newsuburban (Yes/no) & -0.389 & 0.333 \\
\hline
\end{tabular}




\begin{tabular}{|l|l|c|c|}
\hline All together & Q11zeroto 10 & 0.502 & 0.337 \\
\hline Singular & Q11newyrs_exp_11to15 (yes/no) & 0.224 & 0.261 \\
\hline & Q11newyrs_exp_16yrsorover (yes/no) (BL) & & \\
\hline All together & Q12_1 to5 (yes/no) & -0.344 & 0.344 \\
\hline All together & Q12_6to9 (yes/no) & 0.112 & 0.325 \\
\hline & Q12_10 (yes/no) (BL) & & \\
\hline All together & Q1211toover & -0.152 & 0.325 \\
\hline
\end{tabular}


Appendix 13: Single Linear Regression analysis Factor analysis 2 of 3 'Openness Sc

\begin{tabular}{|c|c|c|c|c|c|}
\hline & Variable & B & SE & $t$ & $\underline{\mathbf{p}}$ \\
\hline & Q3. Agerecode & -0.192 & 0.226 & -0.852 & \\
\hline & Q4. Newgender & 0.129 & 0.226 & 0.572 & \\
\hline & Q5. Degree $(y / n)(B L)$ & & & & \\
\hline All together & Q5. Profqual $(y / n)$ & -0.169 & 0.364 & -0.465 & \\
\hline All together & Q5. Masters $(\mathrm{y} / \mathrm{n})$ & -0.241 & 0.272 & -0.886 & \\
\hline All together & Q5. Drorfellow (yes/no) & -0.255 & 0.304 & -0.839 & \\
\hline \multirow[t]{2}{*}{ Singular } & Q6.newprimarydiscipline & 0.103 & 0.07 & 1.465 & \\
\hline & Q6. Nursing B/L & & & & \\
\hline All together & Q6. Psychology (yes/no) & -0.221 & 0.297 & -0.741 & \\
\hline All together & Q6. Psychiatry (yes/no) & -0.714 & 0.306 & -2.331 & \\
\hline \multirow[t]{2}{*}{ All together } & Q6. Therapsw (yes/no) & 0,152 & 0.328 & 0.463 & \\
\hline & Q7newstandalone (yes/no) (BL) & & & & \\
\hline All together & Q7newhubandspoke (yes/no) & -0.127 & 0.261 & -0.484 & \\
\hline All together & $\begin{array}{l}\text { Q7newenhancedcommunityMHT } \\
\text { (yes/no) }\end{array}$ & 0.469 & 0.443 & 1.058 & \\
\hline Singular & Q8.Inpatient (yes/no) & -0.061 & 0.291 & -0.209 & \\
\hline Singular & Q8.Outpatient (yes/no) & 0.061 & 0.247 & 0.248 & \\
\hline Singular & Q8.Casemanagement (yes/no) & -0.084 & 0.487 & -0.172 & \\
\hline Singular & Q8.Daytreatment (yes/no) & 0.106 & 0.323 & 0.328 & \\
\hline Singular & Q9.PracPolPIG (yes/no) & 0.16 & 0.275 & 0.581 & \\
\hline Singular & Q9.PracPoINICE (yes/no) & 0.129 & 0.291 & 0.445 & 0. \\
\hline Singular & $\begin{array}{l}\text { Q9.PracPolWelshPsychological } \\
\text { (yes/no) }\end{array}$ & 0.867 & 0.362 & 2.393 & U \\
\hline \multirow[t]{2}{*}{ Singular } & Q9.PracPolMentalhealth (yes/no) & 0.268 & 0.267 & 1 & \\
\hline & Q10newurban (Yes/no) (BL) & & & & \\
\hline All together & Q10newrural (Yes/no) & -0.056 & 0.273 & -0.204 & \\
\hline All together & Q10newsuburban (Yes/no) & -0.169 & 0.334 & -0.506 & \\
\hline
\end{tabular}




\begin{tabular}{|l|l|c|c|c|c|}
\hline All together & Q11zeroto 10 & 0.393 & 0.334 & 1.179 & 0.24 \\
\hline Singular & Q11newyrs_exp_11to15 (yes/no) & 0.086 & 0.258 & 0.333 & 0.7 \\
\hline & $\begin{array}{l}\text { Q11newyrs_exp_16yrsorover } \\
\text { (yes/no) (BL) }\end{array}$ & & & & \\
\hline All together & Q12_1to5 (yes/no) & 0.183 & 0.34 & 0.539 & 0.59 \\
\hline All together & Q12_6to9 (yes/no) & 0.092 & 0.32 & 0.286 & 0.77 \\
\hline & Q12_10 (yes/no) (BL) & & & & \\
\hline All together & Q12 11toover & 0.054 & 0.32 & 0.167 & 0.86 \\
\hline
\end{tabular}


Appendix 13: EBPAS Score and Linear Regression

\begin{tabular}{|l|l|c|c|c|}
\hline & \multicolumn{1}{|c|}{ Variable } & B & $\underline{\text { SE }}$ & \\
\hline & Q3. Agerecode & -0.164 & 0.108 & \\
\hline & Q4. Newgender & 0.129 & 0.108 \\
& Q5. Degree (y/n) (BL) & & & \\
\hline All together & Q5. Profqual (y/n) & -0.188 & 0.177 & \\
\hline All together & Q5. Masters (y/n) & -0.206 & 0.137 & \\
\hline All together & Q5. Drorfellow (yes/no) & -0.195 & 0.144 & \\
\hline Singular & Q6.newprimarydiscipline & 0.014 & 0.035 & \\
\hline & Q6. Nursing (yes/no) (BL) & & & \\
\hline All together & Q6. Psychology (yes/no) & -0.296 & 0.14 & \\
\hline All together & Q6. Psychiatry (yes/no) & -0.34 & 0.144 \\
\hline All together & Q6. Therapsw (yes/no) & 0.126 & 0.154 & \\
\hline & Q7. Newstandalone (yes/no) (BL) & & & \\
\hline All together & Q7. Newhubandspoke (yes/no) & -0.029 & 0.127 & \\
\hline All together & Q7. NewenhancedcommunityMHT (yes/no) & 0.144 & 0.216 & \\
\hline Singular & Q8. Inpatient (yes/no) & -0.007 & 0.141 & \\
\hline Singular & Q8. Outpatient (yes/no) & 0.001 & 0.119 & \\
\hline Singular & Q8. Casemanagement (yes/no) & 0.041 & 0.235 & \\
\hline Singular & Q8. Daytreatment (yes/no) & 0.001 & 0.156 & \\
\hline Singular & Q9. PracPolPIG (yes/no) & 0.166 & 0.132 & \\
\hline Singular & Q9. PracPoINICE (yes/no) & 0.209 & 0.138 & \\
\hline Singular & Q9. PracPolWelshPsychological (yes/no) & 0.449 & 0.174 & \\
\hline Singular & Q9. PracPoIMentalhealth (yes/no) & 0.03 & 0.13 & \\
\hline & Q10. Newurban (Yes/no) (BL) & & & \\
\hline All together & Q10. Newrural (Yes/no) & 0.031 & 0.131 & \\
\hline All together & Q10. Newsuburban (Yes/no) & -0.153 & 0.16 & \\
\hline All together & Q11zeroto 10 & 0.185 & 0.161 & \\
\hline & & & & \\
\hline
\end{tabular}




\begin{tabular}{|l|l|c|c|} 
All together & Q11. Newyrs_exp_11to15 (yes/no) & 0.132 & 0.124 \\
\hline & $\begin{array}{l}\text { Q11. Newyrs_exp_16yrsorover (yes/no) } \\
\text { (BL) }\end{array}$ & & \\
\hline All together & Q12_1to5 (yes/no) & 0.052 & 0.164 \\
\hline All together & Q12_6to9 (yes/no) & 0.032 & 0.155 \\
\hline & Q12_10 (yes/no) (BL) & & \\
\hline All together & Q1211toover & -0.024 & 0.155 \\
\hline
\end{tabular}




\section{Appendix 14 Models 1 to 4 Primary discipline}

Model 1: Primary discipline and service model (Requirement scale)

Table 12 below shows the result of linear regression analyses. The first model includes the independent variables primary disciplines recodes psychology, psychiatry and therapy / social work with the requirement factor as the dependent variable dependent variable (requirement scale). There are no significant results within this model $\left(F(3,66)=1.554, \mathrm{p}=0.209, \mathrm{R}^{2}\right.$ of 0.066$)$.

Table 12: Model 1 primary discipline main effects

\begin{tabular}{lcccc}
\hline \multicolumn{1}{c}{ Variable } & B & SE & \multicolumn{1}{c}{$\mathrm{t}$} & $\mathrm{p}$ \\
\hline $\begin{array}{l}\text { Constant } \\
\text { Primary } \\
\text { discipline } \\
\text { recode }\end{array}$ & 0.055 & 0.160 & 0.347 & 0.730 \\
$\begin{array}{l}\text { psychology } \\
\begin{array}{l}\text { Primary } \\
\text { discipline }\end{array}\end{array}$ & -0.192 & 0.307 & -0.624 & 0.535 \\
$\begin{array}{l}\text { recode } \\
\text { psychiatry }\end{array}$ & & & & \\
$\begin{array}{l}\text { Primary } \\
\text { discipline } \\
\text { recode } \\
\text { therapsw }\end{array}$ & -0.443 & 0.316 & -1.402 & 0.166 \\
\hline
\end{tabular}




\section{Model 2: primary discipline by service model}

Table 13 below shows the result of linear regression analyses. The second model includes the independent variables primary disciplines recodes psychology, psychiatry and therapy / social work and type of service provided by service model (inpatient) with the requirement factor as the dependent variable. There are no significant results within this model $(F(4,65)=1.292, p$ $=0.282, R^{2}$ of 0.074 ).

Table 13: Model 2 primary discipline by service model (inpatient) main effects

\begin{tabular}{lcccc}
\hline \multicolumn{1}{c}{ Variable } & B & \multicolumn{1}{l}{ SE } & \multicolumn{1}{l}{ t } & \multicolumn{1}{c}{$\mathrm{p}$} \\
\hline $\begin{array}{l}\text { Baseline } \\
\text { Primary } \\
\text { discipline } \\
\text { recode }\end{array}$ & 0.018 & 0.168 & 0.107 & 0.915 \\
$\begin{array}{l}\text { psychology } \\
\begin{array}{l}\text { Primary } \\
\text { discipline }\end{array}\end{array}$ & -0.188 & 0.308 & -0.610 & 0.544 \\
$\begin{array}{l}\text { recode } \\
\text { psychiatry }\end{array}$ & -0.478 & 0.321 & -1.491 & 0.141 \\
$\begin{array}{l}\text { Primary } \\
\text { discipline } \\
\text { recode } \\
\text { therapsw }\end{array}$ & & & & \\
$\begin{array}{l}\text { Types of } \\
\text { services } \\
\text { provided by } \\
\text { your service } \\
\text { model Inpatient }\end{array}$ & 0.408 & 0.341 & 1.199 & 0.235 \\
\hline
\end{tabular}




\section{Model 3: primary discipline by outpatient}

Table 14 below shows the result of linear regression analyses. The third model includes the independent variables primary disciplines recodes psychology, psychiatry and therapy / social work and type of service provided by service model (outpatient) with the requirement factor as the dependent variable. There are no significant results within this model $\left(F(4,65)=1.285, \mathrm{p}=0.285, \mathrm{R}^{2}\right.$ of 0.073).

Table 14: Model 3 primary discipline by service model (outpatient) main effects

\begin{tabular}{lcccc}
\hline \multicolumn{1}{c}{ Variable } & $\mathrm{B}$ & $\mathrm{SE}$ & $\mathrm{t}$ & $\mathrm{p}$ \\
\hline $\begin{array}{l}\text { Baseline } \\
\text { (nurses) }\end{array}$ & 0.017 & 0.169 & 0.102 & 0.919 \\
$\begin{array}{l}\text { Primary } \\
\text { discipline }\end{array}$ & -0.212 & 0.309 & -0.686 & 0.495 \\
$\begin{array}{l}\text { psychology } \\
\begin{array}{l}\text { Primary } \\
\text { discipline }\end{array}\end{array}$ & -0.532 & 0.341 & -1.562 & 0.123 \\
psychiatry & & & & \\
$\begin{array}{l}\text { Primary } \\
\text { discipline }\end{array}$ & 0.393 & 0.340 & 1.155 & 0.252 \\
therapsw & & & & \\
$\begin{array}{l}\text { Types of } \\
\text { services } \\
\text { provided by } \\
\text { your service }\end{array}$ & 0.191 & 0.267 & 0.716 & \\
$\begin{array}{l}\text { model } \\
\text { Outpatient }\end{array}$ & & & & 0.476 \\
\hline
\end{tabular}




\section{Model 4: primary discipline by case management}

Table 15 below shows the result of linear regression analyses. The fourth model includes the independent variables primary disciplines recodes psychology, psychiatry and therapy / social work and type of service provided by service model (case management) with the requirement factor as the dependent variable. There are no significant results within this model $(F(4,65)=1.174, \mathrm{p}=$ $0.330, R^{2}$ of 0.067$)$.

Table 15: Model 4 primary discipline by service model (Case management) main effects

\begin{tabular}{|c|c|c|c|c|}
\hline Variable & $\mathrm{B}$ & SE & $\mathrm{t}$ & $p$ \\
\hline $\begin{array}{l}\text { Baseline } \\
\text { (nurses) }\end{array}$ & -0.095 & 0.502 & -0.189 & 0.851 \\
\hline $\begin{array}{l}\text { Primary } \\
\text { discipline } \\
\text { recode }\end{array}$ & -0.201 & 0.310 & -0.647 & 0.520 \\
\hline $\begin{array}{l}\text { psychology } \\
\text { Primary } \\
\text { discipline } \\
\text { recode } \\
\text { psychiatry }\end{array}$ & -0.426 & 0.323 & -1.317 & 0.192 \\
\hline $\begin{array}{l}\text { Primary } \\
\text { discipline } \\
\text { recode } \\
\text { therapsw }\end{array}$ & 0.384 & 0.342 & 1.121 & 0.266 \\
\hline $\begin{array}{l}\text { Types of } \\
\text { services } \\
\text { provided by } \\
\text { your service } \\
\text { model Case } \\
\text { management }\end{array}$ & 0.159 & 0.505 & 0.316 & 0.753 \\
\hline
\end{tabular}




\section{Model 5: primary discipline by day treatment}

Table 16 below shows the result of linear regression analyses. The fifth model includes the independent variables primary disciplines recodes psychology, psychiatry and therapy / social work and type of service provided by service model (day treatment) with the requirement factor as the dependent variable. There are no significant results within this model $(F(4,65)=1.240, p=0.303$, $R^{2}$ of 0.071$)$.

Table 16: Model 5 primary discipline by service model (day treatment) main effects

\begin{tabular}{|c|c|c|c|c|}
\hline Variable & $B$ & SE & $\mathrm{t}$ & $p$ \\
\hline $\begin{array}{l}\text { Baseline } \\
\text { (nurses) }\end{array}$ & 0.039 & 0.163 & 0.238 & 0.813 \\
\hline $\begin{array}{l}\text { Primary } \\
\text { discipline } \\
\text { psychology }\end{array}$ & -0.220 & 0.312 & -0.704 & 0.484 \\
\hline $\begin{array}{l}\text { Primary } \\
\text { discipline } \\
\text { psychiatry }\end{array}$ & -0.475 & 0.322 & -1.474 & 0.145 \\
\hline $\begin{array}{l}\text { Primary } \\
\text { discipline } \\
\text { therapsw } \\
\text { Types of } \\
\text { services }\end{array}$ & 0.390 & 0.340 & 1.146 & 0.256 \\
\hline $\begin{array}{l}\text { provided by } \\
\text { your service } \\
\text { model Day } \\
\text { treatment }\end{array}$ & 0.195 & 0.332 & 0.587 & 0.559 \\
\hline
\end{tabular}




\section{Appendix 15: Mixed Methods Matrix}

\begin{tabular}{|c|c|c|}
\hline \multirow{2}{*}{$\begin{array}{l}\text { Participant } \\
\text { ID }\end{array}$} & \multicolumn{2}{|c|}{ Summary of findings from: } \\
\hline & Quant study & Qual study \\
\hline 1 & $\begin{array}{l}\text { Gender: Female Requirements (-) } \\
\text { Age: } 45 \text { yrs > (old) Requirements (-) } \\
\text { PhD: Requirements (+) } \\
\text { Psychiatrist: Openness (-); EBPAS (-) } \\
\text { PIG no: Requirements (-) } \\
\text { NICE yes: Requirements (+) } \\
\text { Welsh psych no: Requirements (-) }\end{array}$ & \\
\hline 2 & $\begin{array}{l}\text { Gender: Male Requirements (-) } \\
\text { Age: } 45 \text { yrs < (young) Requirements (+) } \\
\text { Masters: Requirements (-) } \\
\text { Nurse: Openness (+); EBPAS (+) } \\
\text { PIG yes: Requirements (+) } \\
\text { NICE yes: Requirements (+) } \\
\text { Welsh psych no: Requirements (-) }\end{array}$ & $\begin{array}{l}\text { - T1: Clinician and management } \\
\text { (hybrid) two way window } \\
\text { - T2:Outcomes: performance and } \\
\text { quality data }\end{array}$ \\
\hline 3 & $\begin{array}{l}\text { Gender: Female Requirements (+) } \\
\text { Age: } 44 \text { yrs < (young) Requirements (+) } \\
\text { Masters: Requirements (-) } \\
\text { Psychiatrist: Openness (-); EBPAS (-) } \\
\text { PIG no: Requirements (-) } \\
\text { NICE yes: Requirements (+) } \\
\text { Welsh psych no: Requirements (-) }\end{array}$ & - T2: EIP model fidelity 'caseloads' \\
\hline 4 & $\begin{array}{l}\text { Gender: Female Requirements (-) } \\
\text { Age: } 45 \text { yrs > (old) Requirements (-) } \\
\text { Masters: Requirements (-) } \\
\text { Nurse: Openness (+); EBPAS (+) }\end{array}$ & \\
\hline
\end{tabular}




\begin{tabular}{|c|c|c|}
\hline & $\begin{array}{l}\text { PIG yes: Requirements (+) } \\
\text { NICE yes: Requirements (+) } \\
\text { Welsh psych no: Requirements (-) }\end{array}$ & \\
\hline 5 & $\begin{array}{l}\text { Gender: Male Requirements (-) } \\
\text { Age: } 44 \text { yrs < (young) Requirements (+) } \\
\text { PhD: Requirements (+) } \\
\text { Psychologist: EBPAS (-) } \\
\text { PIG yes: Requirements (+) } \\
\text { NICE yes: Requirements (+) } \\
\text { Welsh psych no: Requirements (-) }\end{array}$ & $\begin{array}{l}\text { - T2: Fidelity requires significant } \\
\text { amounts of time } \\
\text { - T2: Practice proficiency } \\
\text { supervision and training barriers t } \\
\text { adopting EBP } \\
\text { - T2: Individual attitude: strong } \\
\text { personalities not motivated in } \\
\text { terms of change culture } \\
\text { - T2: Outcome data: working with } \\
\text { service users realistic data } \\
\text { T2: Communicating data as a } \\
\text { facilitator }\end{array}$ \\
\hline 6 & $\begin{array}{l}\text { Gender: Male Requirements (+) } \\
\text { Age: } 45 \text { yrs > (old) Requirements (-) } \\
\text { Fellowship: Requirements (+) } \\
\text { Psychiatrist: Openness (-); EBPAS (-) } \\
\text { PIG no: Requirements (-) } \\
\text { NICE yes: Requirements (+) } \\
\text { Welsh psych no: Requirements (-) }\end{array}$ & $\begin{array}{l}\text { - T1: What constitutes evidence } \\
\text { - T1: Implies a direct link with } \\
\text { evidence and policy } \\
\text { - T1: Clinician and management } \\
\text { (hybrid) }\end{array}$ \\
\hline 7 & $\begin{array}{l}\text { Gender: Female Requirements (+) } \\
\text { Age: } 44 \text { yrs < (young) Requirements (+) } \\
\text { Degree: Requirements (-) } \\
\text { Psychiatrist: Openness (-); EBPAS (-) } \\
\text { PIG yes: Requirements (+) } \\
\text { NICE yes: Requirements (+) } \\
\text { Welsh psych no: Requirements (-) }\end{array}$ & $\begin{array}{l}\text { T1: Lack of understanding of } \\
\text { specialist practice but attempts no } \\
\text { effort to bridge understanding }\end{array}$ \\
\hline 8 & $\begin{array}{l}\text { Gender: Male Requirements (+) } \\
\text { Age: } 45 \text { yrs > (old) Requirements (-) } \\
\text { PhD: Requirements (+) } \\
\text { Psychologist: EBPAS (-) }\end{array}$ & $\begin{array}{l}\text { - T1: EBP not straight forward notio } \\
\text { - T1: NICE don't have monopoly on }\end{array}$ \\
\hline
\end{tabular}




\begin{tabular}{|c|c|c|}
\hline & $\begin{array}{l}\text { PIG yes: Requirements (+) } \\
\text { NICE yes: Requirements (+) } \\
\text { Welsh psych no: Requirements (-) }\end{array}$ & $\begin{array}{l}\text { what constitutes evidence based } \\
\text { practice } \\
\text { - T2: Real world clinical practice } \\
\text { deviation of models and resource }\end{array}$ \\
\hline 9 & $\begin{array}{l}\text { Gender: Male Requirements (+) } \\
\text { Age: } 45 \text { yrs > (old) Requirements (-) } \\
\text { Masters: Requirements (-) } \\
\text { Nurse: Openness (+); EBPAS (+) } \\
\text { PIG yes: Requirements (+) } \\
\text { NICE yes: Requirements (+) } \\
\text { Welsh psych no: Requirements (-) }\end{array}$ & $\begin{array}{l}\text { - T1: Lack of understanding of } \\
\text { specialist practice } \\
\text { - } \mathrm{T} 1 \text { : People making decisions are } \\
\text { not cognisant of service delivery } \\
\text { - T1: Challenges on how evidence } \\
\text { based practice is delivered } \\
\text { - T2: Standard services operating } \\
\text { like medical model }\end{array}$ \\
\hline 10 & $\begin{array}{l}\text { Gender: Male Requirements (+) } \\
\text { Age: } 45 \text { yrs > (old) Requirements (-) } \\
\text { Professional Qual: Requirements (-) } \\
\text { Nurse: Openness (+); EBPAS (+) } \\
\text { PIG yes: Requirements (+) } \\
\text { NICE yes: Requirements (+) } \\
\text { Welsh psych yes: Requirements (+) }\end{array}$ & \\
\hline 11 & $\begin{array}{l}\text { Gender: Female Requirements (+) } \\
\text { Age: } 44 \text { yrs < (young) Requirements }(+) \\
\text { Degree: Requirements }(-) \\
\text { Nurse: Openness }(+) \text {; EBPAS }(+) \\
\text { PIG yes: Requirements (+) } \\
\text { NICE yes: Requirements (+) } \\
\text { Welsh psych yes: Requirements (+) }\end{array}$ & $\begin{array}{l}\text { - T1: Rationale for early interventio } \\
\text { others don't understand their work } \\
\text { or evidence base supporting it } \\
\text { - T1: Managers must be drawing } \\
\text { upon knowledge themselves }\end{array}$ \\
\hline 12 & $\begin{array}{l}\text { Gender: Female Requirements (+) } \\
\text { Age: } 44 \text { yrs < (young) Requirements }(+) \\
\text { Masters: Requirements }(-) \\
\text { PIG yes: Requirements (+) }\end{array}$ & \\
\hline
\end{tabular}




\begin{tabular}{|c|c|c|}
\hline & $\begin{array}{l}\text { NICE yes: Requirements (+) } \\
\text { Welsh psych yes: Requirements (+) }\end{array}$ & \\
\hline 13 & $\begin{array}{l}\text { Gender: Male Requirements (+) } \\
\text { Age: } 45 \text { yrs > (old) Requirements (-) } \\
\text { Nurse: Openness (+); EBPAS (+) } \\
\text { PIG yes: Requirements (+) } \\
\text { NICE yes: Requirements (+) } \\
\text { Welsh psych yes: Requirements (+) }\end{array}$ & $\begin{array}{l}\text { - T2: Economic climate no funds } \\
\text { protected for EIP. }\end{array}$ \\
\hline 14 & $\begin{array}{l}\text { Gender: Male Requirements (+) } \\
\text { Age: } 45 \text { yrs > (old) Requirements (-) } \\
\text { Nurse: Openness (+); EBPAS (+) } \\
\text { PIG no: Requirements (-) } \\
\text { NICE no: Requirements (-) } \\
\text { Welsh psych no: Requirements (-) }\end{array}$ & $\begin{array}{l}\text { - T1: Individual qualities a colleagu } \\
\text { had to champion EIP } \\
\text { - T2: Outcomes: lobbying for EIP }\end{array}$ \\
\hline 15 & $\begin{array}{l}\text { Gender: Male Requirements (+) } \\
\text { Age: } 45 \text { yrs > (old) Requirements (-) } \\
\text { Psychologist: EBPAS (-) } \\
\text { PIG no: Requirements (-) } \\
\text { NICE no: Requirements (-) } \\
\text { Welsh psych no: Requirements (-) }\end{array}$ & $\begin{array}{l}\text { - T1: Political support within } \\
\text { organisations } \\
\text { - T1: Clinicians to engage with like- } \\
\text { minded individuals at board level } \\
\text { - T2: Practice profiency: Fidelity } \\
\text { difficult area }\end{array}$ \\
\hline 16 & $\begin{array}{l}\text { Gender: Male Requirements (-) } \\
\text { Age: } 44 \text { yrs < (young) Requirements (+) } \\
\text { Psychiatry: Openness (-); EBPAS (-) } \\
\text { PIG yes: Requirements (+) } \\
\text { NICE no: Requirements (-) } \\
\text { Welsh psych no: Requirements (-) }\end{array}$ & $\begin{array}{l}\text { - T2: Individual attitude: resistant to } \\
\text { change }\end{array}$ \\
\hline 17 & $\begin{array}{l}\text { Gender: Male Requirements (-) } \\
\text { Age: } 44 \text { yrs < (young) Requirements (+) } \\
\text { Psychiatrist: Openness (-); EBPAS (-) } \\
\text { PIG no: Requirements (-) } \\
\text { NICE yes: Requirements (+) } \\
\text { Welsh psych yes: Requirements (+) }\end{array}$ & \\
\hline 18 & $\begin{array}{l}\text { Gender: Male Requirements }(-) \\
\text { Age: } 44 \text { yrs < (young) Requirements }(+) \\
\text { Nurse: Openness }(+) \text {; EBPAS }(+)\end{array}$ & $\begin{array}{l}\text { Barriers recruitment issues, staff } \\
\text { shortages }\end{array}$ \\
\hline
\end{tabular}


PIG yes: Requirements (+)

NICE yes: Requirements (+)

Welsh psych yes: Requirements (+) 